\title{
Sobre configurações centrais do problema de $n$ corpos. Configurações centrais planares, espaciais e empilhadas.
}

\author{
Antonio Carlos Fernandes
}

\author{
TESE APRESENTADA \\ $\mathrm{AO}$ \\ Instituto de Matemática e Estatística \\ DA \\ Universidade De SÃo Paulo \\ PARA \\ OBTENÇÃo DO TÍtUlo \\ $\mathrm{DE}$ \\ Doutor EM CIÊNCIAS.
}

\begin{abstract}
Programa: Matemática Aplicada
Orientador: Prof. Dr. Luis Fernando de Osório Mello.
\end{abstract}

Durante o desenvolvimento deste trabalho o autor recebeu auxílio financeiro da CAPES

São Paulo, Dezembro de 2011 


\section{Sobre configurações centrais do problema de $n$ corpos. Configurações centrais planares, espaciais e empilhadas.}

Esta tese contém as correções e alterações sugeridas pela Comissão Julgadora durante a defesa realizada por (Antonio Carlos Fernandes) em 23/11/2011.

O original encontra-se disponível no Instituto de Matemática e Estatística da Universidade de São Paulo.

Comissão Julgadora:

- Prof. Dr. Luis Fernando de Osório Mello (orientador-externo) - UNIFEI.

- Prof. Dr. Clodoaldo Grotta Ragazzo - IME-USP.

- Prof. Dr. Manuel Valentim de Pera Garcia - IME-USP.

- Profa. Dra. Luci Any Francisco Roberto - IBILCE-UNESP.

- Prof. Dr. Eduardo Shirlipe Goes Leandro - DMAT-UFPE. 


\section{Agradecimentos}

Primeiramente, agradeço muito ao Prof. Luis Fernando pela amizade, orientação e todos os ensinamentos que vêm desde a Geometria Analítica no primeiro semestre da graduação.

Agradeço ao Prof. Clodoaldo, à Profa. Helena, aos demais professores e a todo o pessoal das secretarias, por toda a ajuda e cooperação durante meus estudos no IME-USP.

Agradeço à Tatiane, ao Guilherme e à Luísa, por estarem junto a mim nesta empreitada.

Por fim, agradeço aos meus familiares e amigos que direta ou indiretamente contribuíram para a realização deste trabalho. 


\section{Resumo}

No presente trabalho apresentaremos alguns aspectos do problema Newtoniano de $n$ Corpos. Estudaremos o caso de dois corpos, que tem solução direta, embora não seja possível obter todas as variáveis como função do tempo. No caso $n \geq 3$ mostraremos que não existe método para integrar este problema via quadraturas. Podemos tirar apenas algumas informações sobre o caso geral, como a Identidade de Lagrange-Jacobi, o Teorema de Sundman-Weierstrass entre outros.

Veremos alguns casos de soluções particulares, que serão chamadas de soluções homográficas. Nestas soluções a forma geométrica da configuração inicial dos corpos é preservada durante o movimento. Veremos condições necessárias sobre as configurações iniciais para que seja possível obter estas soluções. Mostraremos uma relação existente entre estas soluções particulares e os pontos críticos de uma aplicação, que associa a uma configuração a energia total e o momento angular total do sistema. Nestes vários casos, cairemos numa mesma equação algébrica, que será chamada de equação das configurações centrais. Mostraremos, em seguida, que as equações de configurações centrais são equivalentes a um outro conjunto de equações algébricas, que servem também para calcular as chamadas configurações centrais, porém, com estas equações as simetrias do problema ficam mais claras. Faremos algumas aplicações diretas destas equações algébricas.

Uma subclasse interessante da classe das configurações centrais são as chamadas configurações centrais empilhadas, nas quais um subconjunto próprio dos corpos também forma uma configuração central. Nos dois últimos capítulos veremos alguns exemplos de configurações centrais deste tipo, em especial aquelas onde podemos retirar uma massa e ainda ter uma configuração central.

Palavras-chave: Problema de $n$ Corpos, Solução Homográfica, Configuração Central, Equações de Andoyer, Configurações Centrais Empilhadas. 


\section{Abstract}

In this work we present some aspects of the Newtonian $n$ body problem. We study the case of two bodies, which have a straightforward solution, although we can not get all the variables as functions of the time. For $n \geq 3$ we show that there is no method to integrate this problem by quadratures. We can have just some information about the general case, as the Lagrange-Jacobi's Identity the Sundman-Weierstrass's theorem and others.

We will see some cases of particular solutions, which will be called homographic solutions. In these solutions the geometric shape of initial configuration of the bodies is preserved during the movement. We will see necessary conditions on the initial positions that turn possible to obtain these solutions. We show a relation between these particular solutions and critical points of an application, that associate the total energy and total angular momentum of the system. In these several cases, we will fall in same algebraic equation, which we called of the central configurations equations. We show that the central configurations equations are equivalent to another set of algebraic equations, which are also used to compute the central configurations, but with these equations the symmetries of the problem become clearer. We will make some direct applications these algebraic equations.

An interesting subclass of the class of central configurations are called stacked central configurations, in which a proper subset of the bodies form a central configuration too. In the last two chapters we will see some examples of central configurations of this kind, especially those where we can remove a mass and still have a central configuration.

Keywords: $n$ Body problem, Homographic Solutions, Central Configuration, Andoyer's Equations, Satcked Central Configurations. 


\section{Sumário}

Lista de Figuras

1 Introdução $\quad 1$

2 Problema de Dois Corpos $\quad 5$

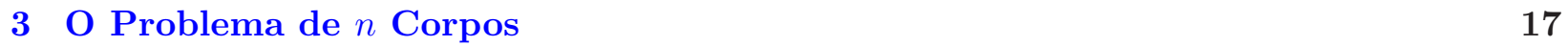

3.1 Equações de Movimento e Integrais Primeiras . . . . . . . . . . . . . . . . . 17

3.2 Não-Integrabilidade do Problema de $n$ corpos . . . . . . . . . . . . . . . . . 20

3.3 Alguns resultados do Problema de $n$ corpos . . . . . . . . . . . . . . . . 22

4 Soluções Particulares do Problema de $n$ Corpos $\quad 31$

4.1 Soluções Homotéticas . . . . . . . . . . . . . . . . . . . . . . . . 31

4.2 Soluções de Equilíbrio Relativo . . . . . . . . . . . . . . . . . . . . . . . 34

4.3 Soluções Homográficas . . . . . . . . . . . . . . . . . . . . . . . . 40

4.4 Pontos Críticos da Superfície Energia-Momento . . . . . . . . . . . . . 47

5 Configurações Centrais $\quad 53$

5.1 Definições e Resultados da Literatura . . . . . . . . . . . . . . . . . . . . . . . . . . . . . . . . 53

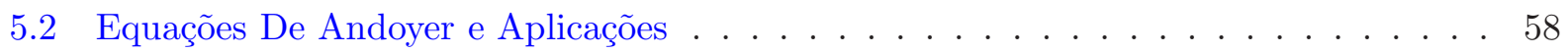

5.3 Configurações Centrais do Tipo Pipa . . . . . . . . . . . . . . . . . . . 68

5.4 Configurações Centrais Empilhadas Parte $1 \ldots \ldots$. . . . . . . . . . . 85

6 Configurações Centrais Empilhadas Parte 2 123

6.1 Configurações Centrais Planares, não Colineares, $(5,1)$-Empilhadas . . . . . . . . 123

6.2 Configurações Centrais Espaciais $(5,1)$-Empilhadas . . . . . . . . . . . . . . . 131

6.3 Casos de Configurações Centrais $(n, 1)$-Empilhadas . . . . . . . . . . . . . . . 141

6.4 "Variação" de uma massa numa Configuração Central . . . . . . . . . . . . . . . . . . 149

6.5 Configurações Centrais Empilhadas Fracas . . . . . . . . . . . . . . . . 153

Referências Bibliográficas $\quad 155$ 
Índice Remissivo 


\section{Lista de Figuras}

2.1 Plano de movimento onde evidenciamos o uso de coordenadas polares. . . . . . . . . . . 7

2.2 Possíveis movimentos com $J \neq 0 . \quad \ldots \ldots \ldots \ldots \ldots \ldots \ldots$

2.3 Órbita em forma de elipse. . . . . . . . . . . . . . . . . . . . . . . 11

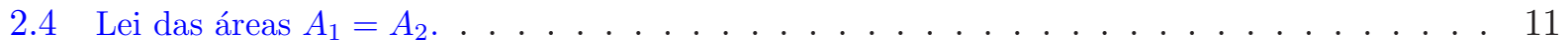

4.1 Configuração colinear de Euler. . . . . . . . . . . . . . . . . . . 33

4.2 Solução de equilíbrio relativo de Euler. . . . . . . . . . . . . . . . . . . . . . . . 39

4.3 Solução de equilíbrio relativo de Lagrange. . . . . . . . . . . . . . . . . . . . . . . 40

4.4 Solução homográfica de Euler. . . . . . . . . . . . . . . . . . . . . . 45

4.5 Solução homográfica de Lagrange. . . . . . . . . . . . . . . . . . . . . 46

4.6 Solução homográfica poligonal. . . . . . . . . . . . . . . . . . . . . . . 46

5.1 Dois exemplos de configurações centrais planares . . . . . . . . . . . . . . . . . . 57

5.2 Exemplo de Roberts: temos configurações centrais para todos os valores positivos de $u$. . . 58

5.3 As duas configurações equiláteras. . . . . . . . . . . . . . . . . . 65

5.4 Configuração central tetraédrica. . . . . . . . . . . . . . . . . . . 65

5.5 Não é configuração central. . . . . . . . . . . . . . . . . . . . . . . . . 66

5.6 Poderia ser configuração central . . . . . . . . . . . . . . . . . . 66

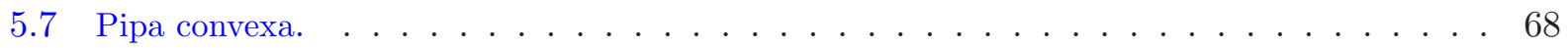

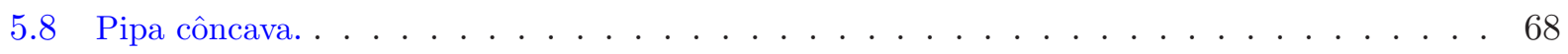

5.9 Configuração central do tipo pipa. . . . . . . . . . . . . . . . . . . . . 69

5.10 Regiões abertas $A_{1}, A_{2}$ e $A_{3} \ldots \ldots \ldots \ldots \ldots \ldots \ldots \ldots$

5.11 Configurações centrais espaciais para 5-corpos. Configuração côncava (a). Configuração

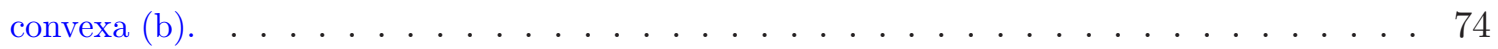

5.12 Coordenadas para o problema com 5 corpos. . . . . . . . . . . . . 76

5.13 Regiões abertas $\mathcal{H}_{1}, \mathcal{H}_{2}$ e $\mathcal{W} \ldots \ldots \ldots \ldots \ldots \ldots$

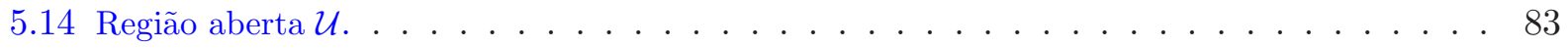

5.15 Configuração de Hampton. . . . . . . . . . . . . . . . . . . . 85

5.16 Curva de configurações centrais exemplo de Hampton. . . . . . . . . . . . . . . 86 
5.17 Configuração espacial de Hampton e Santoprete. . . . . . . . . . . . . . . . . . . 87

5.18 Curvas de configurações centrais exemplo de Hampton e Santoprete. . . . . . . . . . . 88

5.19 Configuração de Mello e Llibre. ～. . . . . . . . . . . . . . . . . . . . . . . . 89

5.20 Segmentos das configurações centrais de Mello e Llibre. . . . . . . . . . . . . . . . . . . 90

5.21 Quatro corpos nos vértices de um tetraedro regular e dois sobre a reta $\mathcal{L}$ que conecta um dos vértices e o centro da face oposta. . . . . . . . . . . . . . . . . . . . 91

5.22 Seis corpos em configuração central espacial. . . . . . . . . . . . . . . . . . . . . 93

5.23 A região $\mathcal{W}_{1}$ e o segmento $I^{1}(G)$ do Teorema 5.4.4 . . . . . . . . . . . . . . . . 109

5.24 A região $\mathcal{W}_{2}$ e o segmento $I^{2}(G)$ do Teorema 5.4.4 . . . . . . . . . . . . . 110

5.25 A região $\mathcal{W}_{3}$ e o segmento $I^{3}(G)$ do Teorema 5.4.4 . . . . . . . . . . . . . . . . 111

5.26 Configuração de Mello, Llibre e Perez-Chavela ～. . . . . . . . . . . . . . . . . . . 112

5.27 Curva configurações centrais de Mello, Llibre e Perez-Chavela . . . . . . . . . . . . . . . 112

5.28 Vetores posição $r_{1}, r_{2}, r_{3}$ e $r_{4}$ nos vértices de $\mathcal{T}_{1}$ e vetores posição $r_{5}, r_{6}$ e $r_{7}$ nos vértices de $\mathcal{E}_{1} .113$

5.29 As regiões $\mathcal{A}_{1}, \mathcal{A}_{2}$ e $\mathcal{A}_{3} \ldots \ldots \ldots \ldots \ldots \ldots$

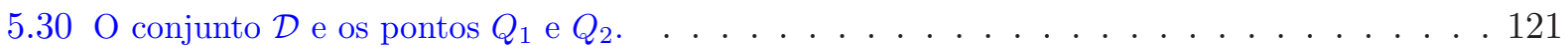

6.1 (a) $r_{1}, r_{2}$ e $r_{5}$ sobre uma reta. (b) $r_{5}$ no exterior de $\mathcal{T}$, mas fora das retas que contém algum

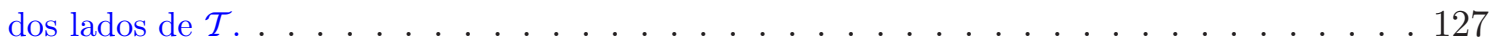

6.2 (a) $r_{1}, r_{2}$ e $r_{5}$ sobre uma reta. (b) $r_{1}, r_{2}, r_{3}$ e $r_{4}$ sobre um semicírculo com centro em $r_{5} \ldots 129$

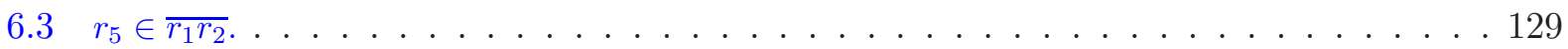

$6.4 r_{1}, r_{2}, r_{3}$ e $r_{4}$ sobre o círculo de raio $d_{6}$ e centro em $r_{5} \ldots \ldots \ldots \ldots$

6.5 Configuração central $(5,1)$-empilhada que deixa uma configuração espacial. . . . . . . . . . 137

6.6 Configuração piramidal. . . . . . . . . . . . . . . . . . . . . 138

6.7 Configuração central $(5,1)$-empilhada que deixa uma configuração planar. . . . . . . . . 139

6.8 Não há posição para $r_{c}$ fora do conjunto colisão. . . . . . . . . . . . . . . . . . 145

6.9 Configuração de Mello, Llibre e Perez-Chavela. . . . . . . . . . . . . . . . . . . 153 


\section{Capítulo 1}

\section{Introdução}

O movimento dos corpos celestes há muito tempo encanta os homens. Portanto, como funciona tal movimento foi uma das primeiras perguntas da dinâmica. Com o intuito de responder a esta pergunta diversos modelos foram criados, por exemplo: O modelo geocêntrico dos gregos, que imaginavam a terra como centro geométrico do universo; O modelo heliocêntrico de Nicolau Copérnico, que pode se visto em [6], que propunha que o Sol é o centro geométrico do universo e que os planetas giravam ao seu redor em órbitas circulares; Um modelo que se adequa bem às observações foi proposto por Johannes Kepler nos trabalhos, Astronomia Nova [15] e Harmonice Mundi [16]. Baseado nas idéias heliocêntricas e nos dados de mais de 14 anos de observação do observatório de Tycho Brahe, Kepler postulou três leis, hoje chamadas de Leis de Kepler, as quais seguem:

1. Primeira Lei: Os Planetas se movem em órbitas elípticas com o Sol num dos focos [15];

2. Segunda Lei: O segmento que vai do Sol ao Planeta varre áreas iguais em tempos iguais [15];

3. Terceira Lei: O período de revolução da órbita é proporcional à potência três meios do eixo maior da elipse [16].

Em seguida, vem o problema de dizer se estas leis podem ser, ou não, obtidas a partir de noções mais fundamentais. Para isto, iniciam-se discussões sobre a dinâmica do movimento planetário e, por conseguinte, tentativas de descrição da lei de interação que regia tal movimento. Com este propósito, Isaac Newton formula em 1666 a Lei da Gravitação Universal e a publica em 1687 no seu Philosophiae Naturalis Principia Mathematica [35]. 
Lei da Gravitação Universal: A interação gravitacional entre dois corpos pode ser expressa por uma força central, atrativa, proporcional ao produto das massas destes corpos e inversamente proporcional ao quadrado da distância entre eles, vide [2].

Assim, em norma, a força que um corpo exerce no outro é dada por

$$
F=\frac{G m_{1} m_{2}}{d^{2}}
$$

onde $m_{1}$ e $m_{2}$ representam as massas dos corpos, $d$ a distância entre eles e $G$ é uma constante de proporcionalidade, que de acordo com medições recentes [21] é dada por

$$
G=(6,67259 \pm 0,00085) \cdot 10^{-11} N \cdot m^{2} \cdot K g^{-2} .
$$

Newton usou esta lei de atração e deduziu as leis de Kepler como teoremas. A lei da gravitação de Newton não trata apenas de interação Sol e algum planeta, mas de corpos massivos interagindo entre si. Assim é colocado o problema de $n$ corpos, para o qual daremos uma formulação precisa para este problema no Capítulo 3.

Problema de $n$ corpos: Dado um sistema isolado no espaço, formado por $n$ corpos com massas $m_{1}, \ldots, m_{n}$ interagindo pela lei da gravitação universal. Qual é a dinâmica das posições destes corpos? [2].

Newton resolveu o caso de dois corpos, Sol e um planeta. Deixando em aberto os casos $n \geq 3$. Muitos dos grandes nomes da astronomia, física e matemática atacaram este problema, por exemplo: Euler obteve soluções colineares para o problema de três corpos, vide [7]; Lagrange obteve soluções para o problema de três corpos, nas quais os corpos permaneciam nos vértices de um triângulo equilátero a cada instante.

Estas soluções obtidas por Euler e Lagrange são soluções particulares para o problema de três corpos. Em 1887 Ernst Heinrich Bruns provou que nenhum método analítico envolvendo as equações algébricas usuais pode resolver o problema de 3-corpos, vide [5]. Veremos o enunciado do Teorema de Bruns no Capítulo 3. Desta forma, o problema de três corpos não é integrável via quadraturas. 
Do mesmo modo o problema de $n$ corpos não é integrável via quadraturas para $n \geq 3$. Tal fato, torna importantes quaisquer soluções particulares. Um caso interessante de solução particular do problema de $n$ corpos são as soluções homográficas, nas quais a dinâmica das $n$ massas é dada por rotações e homotetias, centradas no centro de massa do sistema. As primeiras soluções homográficas, foram obtidas por Euler no caso colinear de três corpos, como descrito acima.

Como veremos no Capítulo 4, as soluções homográficas estão relacionadas com as configurações centrais, vide os trabalhos de Vidal e Renildo em [45]. Estas são configurações particulares dos corpos nas quais a aceleração de cada corpo, num instante fixo de tempo, é proporcional ao vetor posição deste corpo no referencial do centro de massa. Para as definições corretas veja o Capítulo 5.

Existem diversas perguntas a serem respondidas neste contexto. Uma delas é o sexto da lista de Smale de problemas matemáticos para o século XXI, em [40]. Smale pergunta se dadas $n$ massas positivas no plano, é finito o número de classes de configurações centrais formadas por estas massas? Esta questão foi respondida por Hampton e Moeckel para o caso $n=4$, em [12]. Recentemente, uma resposta parcial para o caso $n=5$ foi dada por Albouy e Kaloshin em [1], no qual os autores mostram que para a maioria de escolhas de cinco massas temos um número finito de classes de configurações centrais.

Apesar destes avanços, muito pouco se conhece sobre as propriedades das configurações centrais em geral. No Capítulo 4 veremos que estas configurações estão relacionadas com as superfícies de energia momento do problema de $n$ corpos. No Capítulo 5 veremos alguns exemplos de configurações centrais consagrados na literatura, bem como alguns recentes, veremos que resultados gerais são difíceis pela complexidade das equações algébricas envolvidas.

Dentro da classe das configurações centrais uma subclasse interessante são as chamadas configurações centrais empilhadas que são configurações centrais que possuem um subconjunto próprio das massas que também está em configuração central. Estas classes foram introduzidas por Hampton, vide [13]. Neste trabalho Hampton mostra a existência de uma classe de configurações centrais de cinco corpos que tem um subconjunto de três corpos que também forma uma configuração central. 
No Capítulo 5, estudaremos alguns casos de configurações centrais empilhadas. Dentre elas uma generalização para o espaço da configuração colocada por Hampton no trabalho citado acima. Estudaremos também uma questão colocada por Hampton, em [13], a qual é

Além do caso colinear simétrico e do caso quadrado com massas iguais nos vértices e uma massa arbitrária na interseção das diagonais do quadrado, existe mais alguma configuração central empilhada de cinco massas na qual um subconjunto de quatro massas também forme uma configuração central?

A resposta é não e a mostraremos no Capítulo 6. Neste Capítulo também será feito um estudo sobre o caso geral de uma configuração central, na qual podemos retirar uma massa e ainda ter uma configuração central.

Primeiramente, no Capítulo 2 vamos resolver um problema de dois corpos mostrando que a lei da gravitação Newtoniana implica nas leis de Kepler e também a recíproca. 


\section{Capítulo 2}

\section{Problema de Dois Corpos}

Neste Capítulo vamos tratar o problema de dois corpos interagindo gravitacionalmente. Vamos considerar o problema no contexto abordado por Newton e Kepler, que é a interação dos Planetas com o Sol. Vamos enunciar e provar o seguinte teorema.

Teorema 2.0.1 Considere um sistema formado pelo Sol e um outro planeta qualquer e que a interação entre eles seja independente da velocidade. Então o movimento deste planeta é dado pelas leis de Kepler se, e somente se, a interação é dada na forma da lei da gravitação universal de Newton.

Prova: Mostraremos primeiro a recíproca, isto é, a lei da gravitação universal implica nas leis de Kepler. Suponha que seja válida a lei da gravitação universal. Considerando como partículas o Sol, com massa $m_{1}$, e o planeta, com massa $m_{2}$, com posições dadas por $q_{1}, q_{2} \in \mathbb{R}^{3}$, respectivamente. Não vamos considerar o caso da colisão. Assim, temos a seguinte Lagrangiana para o sistema

$$
L:\left(\mathbb{R}^{6}-\{0\}\right) \times\left(\mathbb{R}^{6}\right) \rightarrow \mathbb{R}
$$

onde

$$
L\left(q_{1}, q_{2}, \dot{q_{1}}, \dot{q_{2}}\right)=\frac{1}{2}\left(m_{1} \dot{q}_{1}^{2}+m_{2} \dot{q}_{2}^{2}\right)+\frac{G m_{1} m_{2}}{\left|q_{1}-q_{2}\right|} .
$$


Mudando para coordenadas de Jacobi, ela se escreve como

$$
L\left(\xi_{0}, \xi_{1}, \dot{\xi_{0}}, \dot{\xi_{1}}\right)=\left(\frac{m_{1}+m_{2}}{2}\right) \dot{\xi}_{0}^{2}+\frac{m_{1} m_{2}}{2\left(m_{1}+m_{2}\right)}{\dot{\xi_{1}}}^{2}+\frac{G m_{1} m_{2}}{\left|\xi_{1}\right|}
$$

onde

$$
\begin{gathered}
\xi_{0}=\frac{m_{1} q_{1}+m_{2} q_{2}}{m_{1}+m_{2}}, \\
\xi_{1}=q_{1}-q_{2} .
\end{gathered}
$$

A Lagrangiana em (2.1) conduz a uma equação diferencial em $\mathbb{R}^{12}$. Para cada corpo devemos determinar uma 6-upla, na qual três coordenadas são posições e as outras velocidades. Como $\xi_{0}$ é cíclica temos

$$
\xi_{0}(t)=\xi_{0}(0)+t \dot{\xi}_{0}(0)
$$

donde precisamos estudar apenas a Lagrangiana

$$
L=\frac{m_{1} m_{2}}{2\left(m_{1}+m_{2}\right)} \dot{\xi}_{1}^{2}+\frac{G m_{1} m_{2}}{\left|\xi_{1}\right|}
$$

Vemos que a Lagrangiana (2.2) conduz a uma equação diferencial em $\mathbb{R}^{6}$ da seguinte forma

$$
\frac{m_{1} m_{2}}{m_{1}+m_{2}} \ddot{\xi}_{1}+G m_{1} m_{2} \frac{\xi_{1}}{\left|\xi_{1}\right|^{3}}=0 .
$$

Como estamos falando do Sol interagindo com um outro planeta temos $m_{1}>>m_{2}$, o que para o caso Sol e Terra por exemplo é bastante razoável, uma vez que estima-se que no sistema solar a soma das massas de todos os planetas e asteróides corresponda a 0,2\% da massa do Sol, vide [41], daí podemos fazer

$$
\ddot{\xi_{1}}+G m_{1} \frac{\xi_{1}}{\left|\xi_{1}\right|^{3}}=0
$$

Com isto estaremos impondo que o centro de massa do sistema, praticamente, coincida com o do Sol. 
Note que na Lagrangiana (2.2), não há dependência explícita do tempo e o potencial $V$ depende unicamente das distâncias relativas entre os corpos. Assim, considere o enunciado o seguinte teorema, cuja prova pode ser vista em [20].

Teorema 2.0.2 Suponha que a Lagrangiana de um sistema isolado seja $\mathcal{L}$. Pelos postulados de Newton o tempo flui uniformemente e o espaço é homogêneo e isotrópico, ou seja, a Lagrangiana não depende explicitamente do tempo e o potencial depende apenas das coordenadas. Então, associado a estes fatos, temos as seguintes leis de conservação.

1. A homogeneidade do tempo implica na conservação da energia;

2. A homogeneidade do espaço implica na conservação do momento linear total do sistema;

3. A isotropia do espaço implica na conservação do momento angular total do sistema.

Assim o movimento da órbita da equação (2.3) se dá num plano fixo, ortogonal ao vetor momento angular. Vamos usar coordenadas polares sobre este plano, conforme indicado na figura 2.1.

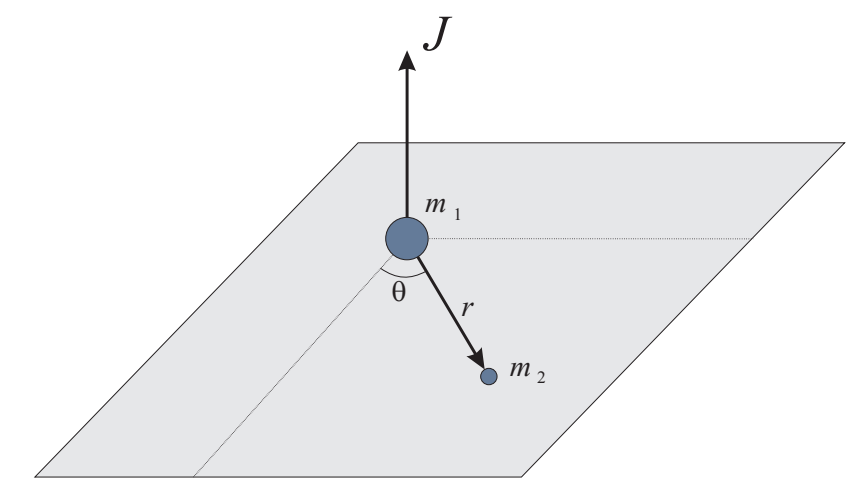

Figura 2.1: Plano de movimento onde evidenciamos o uso de coordenadas polares.

Com a observação da conservação do momento angular vemos que a equação (2.3) é na verdade uma equação diferencial em $\mathbb{R}^{4}$. Usando coordenadas polares para a posição, temos

$$
\xi_{1}=(r \cos \theta, r \operatorname{sen} \theta) .
$$


O módulo do momento angular, que é conservado, em coordenadas polares fica dado por

$$
J=m_{2} r^{2} \dot{\theta}
$$

Aqui usaremos $J$, também para denotar a norma do momento angular, pois não haverá ambiguidades, uma vez o movimento é planar.

A energia cinética fica dada por

$$
T=\frac{m_{2}}{2}\left(\dot{r}^{2}+r^{2} \dot{\theta}^{2}\right)
$$

Juntando as equações (2.4) e (2.5) à definição da energia mecânica do sistema, que também se conserva visto que a Lagrangiana (2.2) não tem dependência explícita no tempo, temos

$$
E=\frac{m_{2} \dot{r}^{2}}{2}+\frac{J^{2}}{2 m_{2} r^{2}}-\frac{G m_{1} m_{2}}{r}
$$

onde usamos

$$
\dot{\theta}=\frac{J}{m_{2} r^{2}}=\frac{d \theta}{d t}
$$

Voltemos nossa atenção à equação (2.4), nesta vemos que $\dot{\theta}$ varia com $|r|$ e não muda de sinal durante o movimento, pois é razoável supor que $J \neq 0$, assim pelo Teorema da função implícita podemos fazer $\theta=\theta(r)$. Desta feita, podemos usar as equações (2.6) e (2.7) para fazer

$$
\dot{r}=\frac{d r}{d t}=\sqrt{\frac{2}{m_{2}}\left(E-\frac{J^{2}}{2 m_{2} r^{2}}+\frac{G m_{1} m_{2}}{r}\right)}=\frac{d r}{d \theta} \frac{J}{m_{2} r^{2}},
$$

donde

$$
d \theta=\frac{J d r}{r^{2} \sqrt{2 m_{2} E-\frac{J^{2}}{r^{2}}+\frac{2 k}{r}}}
$$


onde $k=G m_{1} m_{2}^{2}$. Integrando esta expressão obtemos, a menos de uma constante aditiva,

$$
\theta(r)=\arccos \left(\frac{J / r-k / J}{\sqrt{2 m_{2} E+\frac{k^{2}}{J^{2}}}}\right) .
$$

Invertendo esta relação obtemos

$$
r(\theta)=\frac{\rho}{1+\varepsilon \cos \theta},
$$

com

$$
\rho=\frac{J^{2}}{k} \quad, \quad \varepsilon=\sqrt{2 m_{2} E+\frac{k^{2}}{J^{2}}} .
$$

A expressão (2.10) representa a equação de uma elipse em coordenadas polares, vide figura 2.3, desde que $0<\varepsilon<1$. Isto mostra parcialmente a validade da primeira lei de Kepler. Para ver que a condição $0<\varepsilon<1$ é verificada podemos analisar como $\varepsilon$ varia com a energia. Para isto devemos analisar os gráficos do chamado potencial efetivo,

$$
V_{e f}=\frac{J^{2}}{2 m_{2} r^{2}}-\frac{G m_{1} m_{2}}{r}
$$

Vide figura 2.2 abaixo

Temos que observar empiricamente que no caso de interesse o movimento se dá numa região confinada, isto é com energia negativa. Para valores positivos de energia, $(\varepsilon>1)$, temos ramos de hipérboles e para o valor nulo, $(\varepsilon=1)$, temos uma parábola, ambas são soluções ilimitadas. Portanto, não representam as observações.

Passemos agora ao segundo postulado de Kepler e mostremos que este decorre diretamente da conservação da norma do momento angular. Para a trajetória elíptica descrita acima teremos a seguinte equação para o cálculo do elemento de área, $\mathcal{A}$, varrida pelo vetor posição

$$
d \mathcal{A}=\frac{r^{2} d \theta}{2}=\frac{r^{2}}{2} \frac{d \theta}{d t} d t
$$




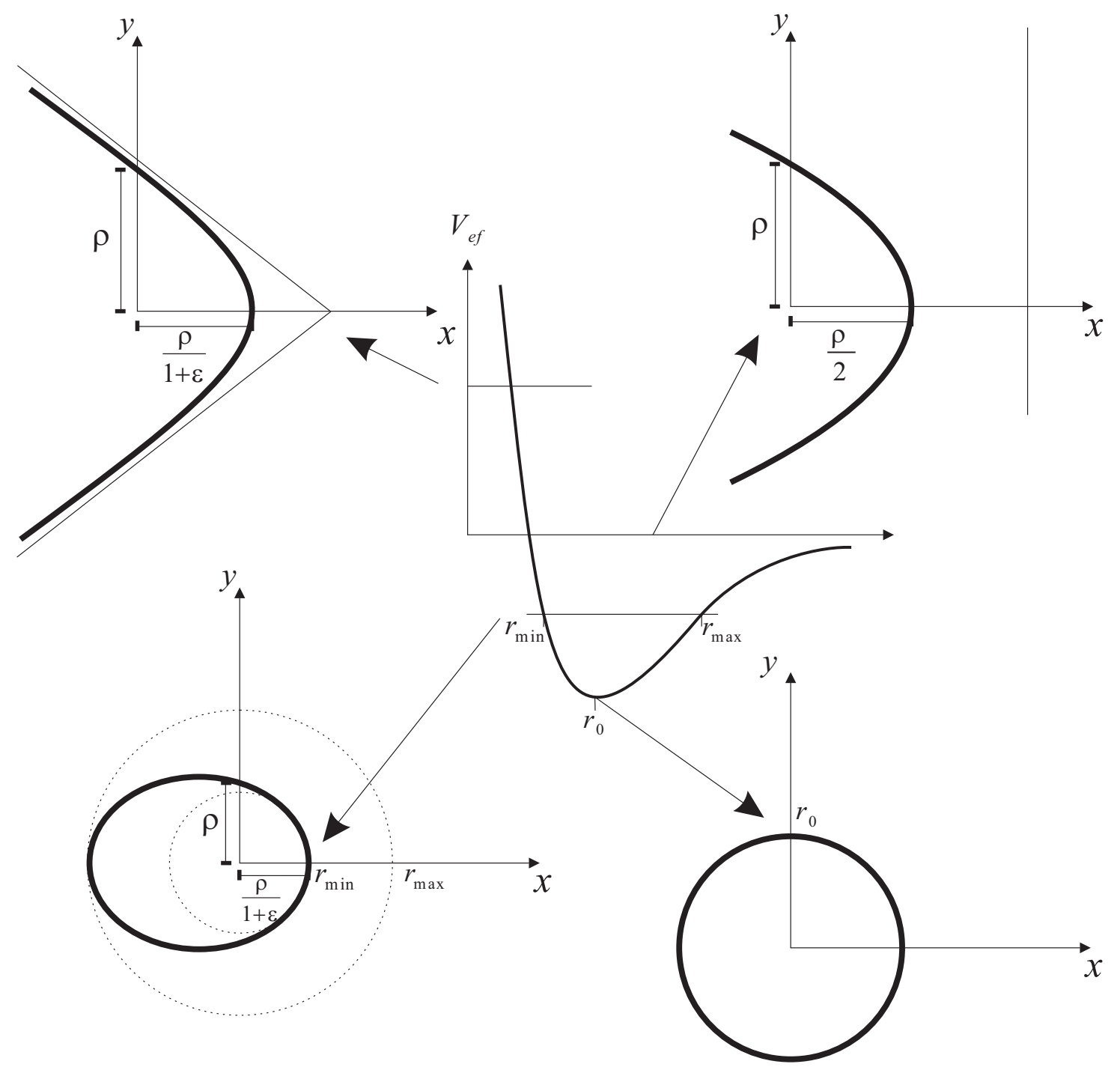

Figura 2.2: Possíveis movimentos com $J \neq 0$.

ou usando a equação (2.4),

$$
d \mathcal{A}=\frac{J}{2} d t
$$

Como $J$ é conservado a área $\mathcal{A}$ é função linear do tempo e isto implica que para tempos iguais teremos áreas iguais. Disto decorre a segunda lei de Kepler, que é conhecida como lei das áreas, vide figura 2.4 .

Observação 2.0.3 Um fato interessante a se mencionar neste ponto é a interpretação de Kepler 


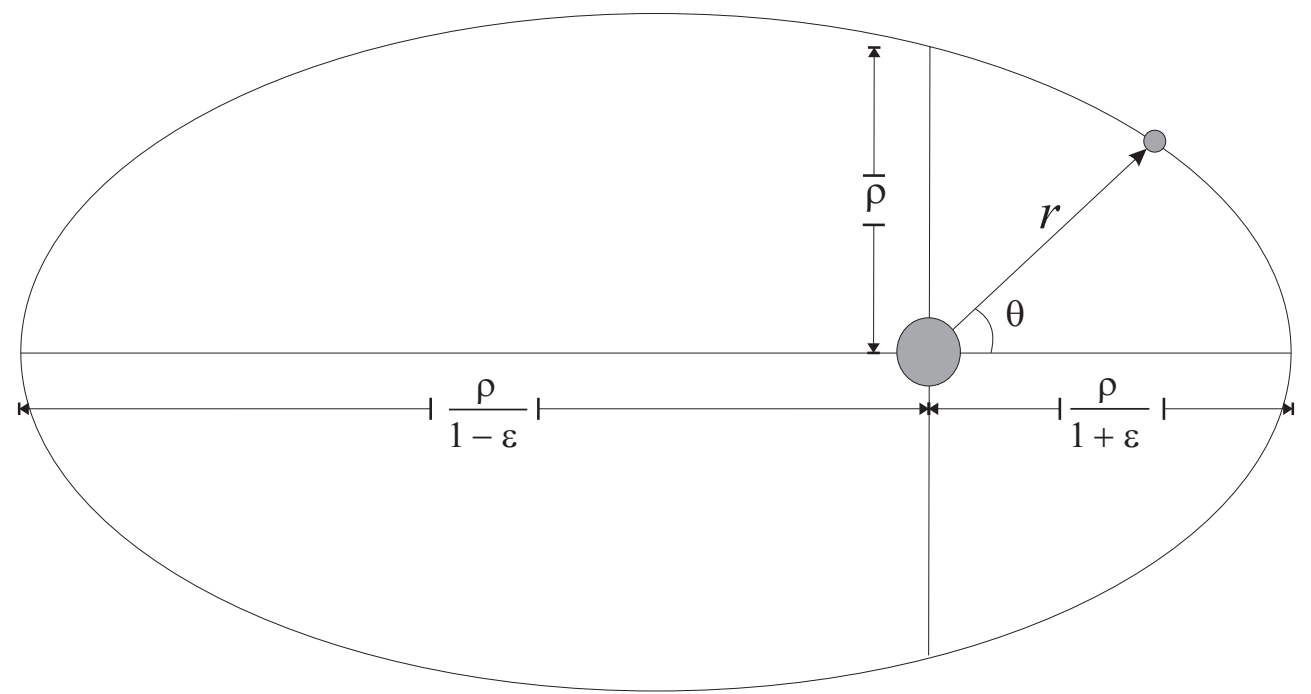

Figura 2.3: Órbita em forma de elipse.

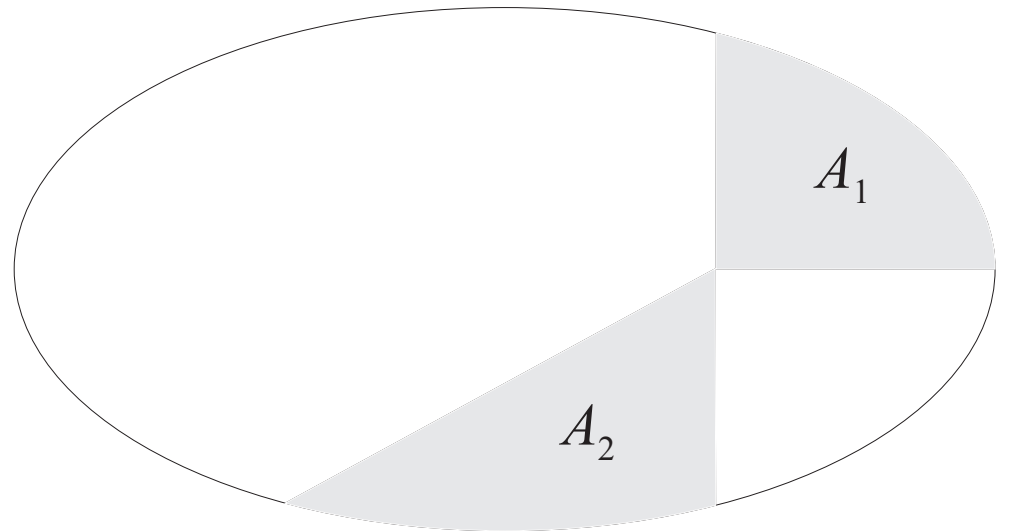

Figura 2.4: Lei das áreas $A_{1}=A_{2}$.

acerca dessa lei, [15]. Uma vez que ele não conhecia o conceito de inércia ele acreditava que o Sol impelia uma força lateral inversamente proporcional à distância sobre os Planetas, (entenda-se por força lateral a componente tangencial que estaria na direção do vetor velocidade). Bastante diferente da interpretação atual.

Para a terceira lei de Kepler consideremos a equação (2.10) e passemo-la para coordenadas cartesianas $(r \cos \theta, r \operatorname{sen} \theta)=(x, y)$. Com tal procedimento teremos

$$
\frac{\left(1-\varepsilon^{2}\right)^{2}}{\rho^{2}}\left(\frac{\rho \varepsilon}{1-\varepsilon^{2}}+x\right)^{2}+\left(\frac{1-\varepsilon^{2}}{\rho^{2}}\right) y^{2}=1 .
$$


A forma padrão para a equação da elipse em coordenadas cartesianas é dada por

$$
\frac{(a \varepsilon+x)^{2}}{a^{2}}+\frac{y^{2}}{b^{2}}=1
$$

Fazendo a correspondência desta com a equação (2.12), vemos que

$$
a=\frac{\rho}{1-\varepsilon^{2}}
$$

$\mathrm{e}$

$$
b=\frac{\rho}{\sqrt{1-\varepsilon^{2}}} .
$$

Sendo que $a$ é o semi-eixo maior da elipse e $b$ o semi-eixo menor. Sabendo que a área total da elipse é dada por

$$
\mathcal{A}=\pi a b
$$

e considerando a expressão (2.11), temos

$$
\mathcal{A}(t)=\frac{J}{2} t
$$

de tal forma que a área total será

$$
\mathcal{A}=\frac{J}{2} \tau
$$

onde $\tau$ representa o período de revolução da órbita elíptica.

Tomando os resultados das expressões (2.13) e (2.14), temos

$$
2 \pi a b=J \tau
$$

inserindo nesta as expressões para os semi-eixos nos vem

$$
\tau=\frac{2 \pi}{J} \frac{\rho}{\left(1-\varepsilon^{2}\right)} \frac{\rho}{\sqrt{1-\varepsilon^{2}}}=\frac{2 \pi \rho^{1 / 2}}{J} \frac{\rho^{3 / 2}}{\left(1-\varepsilon^{2}\right)^{3 / 2}} .
$$


Portanto, com uma constante

$$
C=\frac{2 \pi}{\sqrt{k}}
$$

temos

$$
\tau=C a^{3 / 2}
$$

que é a terceira lei de Kepler.

Observação 2.0.4 Para chegarmos à expressão (2.10) fizemos uso de um método, que consistia em buscar as integrais primeiras do problema e usá-las para reduzir os graus de liberdade do sistema. Assim um sistema inicialmente em $\mathbb{R}^{12}$, foi posto em $\mathbb{R}^{6}$ usando as seis quantidades conservadas do momento linear. Em seguida, usando a conservação da norma, da direção e do sentido do momento angular total levou-se o sistema para $\mathbb{R}^{3}$. Por fim, usamos a conservação da energia total do sistema para obter uma equação em $\mathbb{R}^{2}$ e resolver para $r$ em função de $\theta$. Em verdade, usou-se o fato de existirem 10 integrais primeiras no problema de dois corpos para reduzir o número de graus de liberdade do sistema de doze para dois.

Vamos mostrar agora que as leis de Kepler implicam na lei da gravitação universal de Newton.

Considere que sejam válidas as leis de Kepler para o movimento planetário, assim por hipótese temos que o movimento é planar. Por conseguinte, podemos usar coordenadas polares para descrever tal movimento. Tomando a expressão da aceleração em coordenadas polares obtemos para um planeta de massa $m_{p}$ as seguintes expressões

$$
\left\{\begin{array}{l}
m_{p}\left(\ddot{r}-r \dot{\theta}^{2}\right)=F \\
2 \dot{r} \dot{\theta}+r \ddot{\theta}=0
\end{array}\right.
$$

A segunda equação de (2.15) é equivalente à conservação do momento angular, podemos multiplicar por $r$ em ambos os lados e obter

$$
2 r \dot{r} \dot{\theta}+r^{2} \ddot{\theta}=\frac{d}{d t}\left(r^{2} \dot{\theta}\right)=0
$$


daí

$$
m_{p} r^{2} \dot{\theta}=J=\text { constante. }
$$

Como podemos ver a função $\theta$ não troca de sinal durante o movimento, isso implica que pelo Teorema da Função Implícita podemos encontrar $r=r(\theta)$. Usando a regra da cadeia para derivação, temos

$$
\frac{d}{d \theta}\left(\frac{1}{r}\right)=-\frac{\dot{r}}{J}
$$

e ainda

$$
\frac{d^{2}}{d \theta^{2}}\left(\frac{1}{r}\right)=-\frac{\ddot{r}}{\dot{\theta} J}
$$

Juntando a equação (2.17) com a primeira equação de (2.15), temos

$$
\frac{d^{2}}{d \theta^{2}}\left(\frac{1}{r}\right)+\left(\frac{1}{r}\right)=-\frac{F r^{2}}{m_{p} J^{2}} .
$$

Esta expressão é conhecida como fórmula de Binet, e serve para determinar a órbita se conhecemos a interação ou a interação se conhecemos a órbita. Aqui usaremos seu segundo propósito:

$$
F=-\frac{m_{p} J^{2}}{r^{2}}\left[\frac{d^{2}}{d \theta^{2}}\left(\frac{1}{r}\right)+\left(\frac{1}{r}\right)\right]
$$

Partimos agora da expressão de uma elipse em coordenadas polares para a órbita, pois assumimos a priori a primeira lei de Kepler. Donde

$$
\frac{1}{r}=\frac{1+\varepsilon \cos \theta}{\rho}
$$

Usando esta última na equação (2.18), temos

$$
F=-\frac{m_{p} J^{2}}{r^{2}}\left[-\frac{\varepsilon \cos \theta}{\rho}+\frac{1+\varepsilon \cos \theta}{\rho}\right]
$$

o que nos dá

$$
F=-\frac{m_{p} J^{2}}{\rho} \frac{1}{r^{2}}
$$


Para que a expressão (2.19) nos dê a lei da gravitação resta mostrar que $J^{2} / \rho$ é constante para todos os planetas. Para isto considere a segunda lei de Kepler, isto é, tome a expressão (2.16) para calcular a área da elipse. Assim, nos vem

$$
\frac{d \mathcal{A}}{d t}=\frac{J}{2}
$$

Com esta expressão podemos obter o período de revolução da órbita fazendo

$$
\tau=\frac{\mathcal{A}}{d \mathcal{A} / d t}=\frac{2 \pi c_{1} c_{2}}{J}
$$

onde $c_{1}$ e $c_{2}$ são, respectivamente, o semi-eixo maior e o semi-eixo menor da elipse. Nas relações da elipse é fácil ver que $\rho=c_{2}{ }^{2} / c_{1}$. Tomando o quadrado da equação (2.20) e dividindo por $c_{1}^{3}$, temos a seguinte expressão

$$
\frac{\tau^{2}}{c_{1}^{3}}=\frac{4 \pi^{2} c_{2}^{2}}{c_{1} J^{2}}=\frac{4 \pi^{2} \rho}{J^{2}}
$$

Finalmente, considerando terceira lei de Kepler, vemos que o lado direito da expressão (2.21) deve ser uma constante para todos os planetas. Desta feita temos que a expressão (2.19) é equivalente à lei da gravitação universal de Newton [9].

Agora que vimos uma solução para o caso de dois corpos, podemos nos perguntar sobre soluções do problema geral com $n$ massas positivas interagindo gravitacionalmente. Como veremos, este é um problema muito mais complicado de ser atacado. 


\section{Capítulo 3}

\section{O Problema de $n$ Corpos}

Neste Capítulo vamos fazer uma breve apresentação do problema geral de $n$ massas interagindo gravitacionalmente. Este Problema surge como pergunta natural após os trabalhos de Newton.

\subsection{Equações de Movimento e Integrais Primeiras}

Considere um sistema isolado formado por $n$ partículas de massas $m_{1}, m_{2}, \ldots, m_{n}$ localizadas pelas coordenadas $q_{1}, q_{2}, \ldots, q_{n}$, respectivamente. Considere, $q_{i} \in \mathbb{R}^{3}$ com norma e produto interno usuais.

Suponha que a interação sobre a $i$-ésima partícula devido à ação da $j$-ésima seja dada pela Lei da Gravitação Universal de Newton, conforme enunciado no Capítulo 2. Assim, as equações de movimento serão dadas por

$$
m_{i} \ddot{q}_{i}=-\sum_{j=1, j \neq i}^{n} m_{i} m_{j} \frac{q_{i}-q_{j}}{q_{i j}^{3}}
$$

onde $q_{i j}=\left|q_{i}-q_{j}\right|$ e a constante de gravitação é tomada igual a unidade, o que não ocasiona perda de generalidade para os assuntos tratados no texto. Daqui por diante, quando for citado "Problema Newtoniano de $n$ corpos" entenda-se $n$ partículas interagindo gravitacionalmente com todas as hipóteses acima.

É fácil notar que expressão (3.1) não está bem definida quando há sobreposição de corpos. Isto sugere a seguinte definição. 
Definição 3.1.1 No problema Newtoniano de n corpos, chamaremos de conjunto colisão o seguinte conjunto

$$
\triangle=\bigcup_{i \neq j} \triangle_{i j}
$$

onde

$$
\triangle_{i j}=\left\{Q=\left(q_{1}, \ldots, q_{n}\right) \in \mathbb{R}^{3 n} / q_{i}=q_{j}, i \neq j\right\}
$$

Assim, considere $X=\mathbb{R}^{3 n} \backslash \triangle$ como espaço de configurações do sistema. Sobre $X$ a expressão do lado direito da equação (3.1) é analítica. Não é excluído o caso das soluções com possíveis colisões. Serão tomadas condições iniciais fora do conjunto colisão para garantir existência de soluções, vide seção 3.2 .

Seguem algumas definições que serão importantes no decorrer do Capítulo.

Definição 3.1.2 No problema Newtoniano de $n$ corpos, chamaremos de massa total do sistema a seguinte quantidade escalar

$$
\mu=\sum_{i=1}^{n} m_{i} .
$$

Definição 3.1.3 No problema Newtoniano de n corpos, chamaremos de centro de massa do sistema o seguinte vetor

$$
C=\frac{1}{\mu} \sum_{i=1}^{n} m_{i} q_{i} .
$$

Definição 3.1.4 No problema Newtoniano de $n$ corpos, chamaremos momento linear total do sistema o seguinte vetor

$$
P=\sum_{i=1}^{n} m_{i} \dot{q}_{i} .
$$

Definição 3.1.5 No problema Newtoniano de $n$ corpos, chamaremos energia cinética total do sistema a seguinte quantidade escalar

$$
T=\frac{1}{2} \sum_{i=1}^{n} m_{i}\left|\dot{q}_{i}\right|^{2} .
$$

Definição 3.1.6 No problema Newtoniano de $n$ corpos, chamaremos energia potencial do sistema a seguinte quantidade escalar

$$
V=-\sum_{i<j} \frac{m_{i} m_{j}}{q_{i j}}
$$


Definição 3.1.7 No problema Newtoniano de $n$ corpos, chamaremos energia total do sistema a seguinte quantidade escalar

$$
E=T+V=\frac{1}{2} \sum_{i=1}^{n} m_{i}\left|\dot{q}_{i}\right|^{2}-\sum_{i<j} \frac{m_{i} m_{j}}{q_{i j}} .
$$

Definição 3.1.8 No problema Newtoniano de $n$ corpos, chamaremos momento angular total do sistema o seguinte vetor

$$
J=\sum_{i=1}^{n} m_{i} q_{i} \wedge \dot{q}_{i}
$$

onde $\wedge$ denota o produto vetorial de $\mathbb{R}^{3}$.

Com estas definições, seja $\mathcal{L}: T X \rightarrow \mathbb{R}$ a Lagrangiana do sistema

$$
\mathcal{L}=T-V=\frac{1}{2} \sum_{i=1}^{n} m_{i}\left|\dot{q}_{i}\right|^{2}+\sum_{i<j} \frac{m_{i} m_{j}}{q_{i j}} .
$$

Note que em $\mathcal{L}$, não há dependência explícita do tempo e o potencial $V$ depende unicamente das distâncias relativas entre os corpos. Assim, no caso do problema Newtoniano de $n$ corpos o Teorema 2.0.2 fornece-nos 10 integrais de movimento algébricas com respeito às posições e velocidades, da seguinte maneira:

- Uma quantidade da conservação da energia,

$$
E=T+V=E_{0}
$$

- Três quantidades da conservação do momento angular total,

$$
J=J_{0}
$$

- Três quantidades da conservação do momento linear total,

$$
P=P_{0}
$$


- Desta última vemos que o vetor do centro de massa é escrito da seguinte maneira,

$$
C=C_{0}+t \frac{P_{0}}{\mu}
$$

com $C_{0}$ constante, donde temos mais três quantidades.

Uma vez que no problema de $n$ corpos há $6 n$ graus de liberdade, como pode ser visto em (3.1), para integrá-lo seriam necessárias $6 n-1$ integrais de movimento algebricamente independentes. Em princípio, é razoável procurar por outras integrais primeiras, além das dez listadas acima. Porém, como será visto na próxima seção, tal procedimento não funciona.

\subsection{Não-Integrabilidade do Problema de $n$ corpos}

Usando notação compactada, é possível escrever a equação (3.1) na seguinte forma

$$
M \ddot{Q}=-\nabla V,
$$

onde

$$
M=\operatorname{diag}\left[m_{1}, m_{1}, m_{1}, m_{2}, m_{2}, m_{2}, \ldots, m_{n}\right]
$$

é uma matriz diagonal $3 n \times 3 n$,

$$
Q=\left(q_{1}, q_{2}, \ldots, q_{n}\right)^{t}
$$

é um vetor de $\mathbb{R}^{3 n}$,

$$
\nabla=\left(\nabla_{1}, \nabla_{2}, \ldots, \nabla_{n}\right)^{t}
$$

é um operador diferencial, com $\nabla_{k}$ sendo o gradiente nas coordenadas do $k$-ésimo corpo.

Tomemos condições iniciais da seguinte forma

$$
Q(0) \in\left\{\mathbb{R}^{3 n} \backslash \triangle\right\}
$$

e

$$
\dot{Q}(0) \in \mathbb{R}^{3 n}
$$


É possível pensar no problema de $n$ corpos como um problema de valor inicial,

$$
\left\{\begin{array}{l}
\dot{S}=F(S) \equiv\left(\dot{Q},-M^{-1} \nabla V\right) \\
S(0)=(Q(0), \dot{Q}(0)) \in\left\{\mathbb{R}^{3 n} \backslash \triangle\right\} \times \mathbb{R}^{3 n},
\end{array}\right.
$$

onde $S=(Q, \dot{Q}) \in \mathbb{R}^{6 n}$ e $F: \mathbb{R}^{3 n} \times\left\{\mathbb{R}^{3 n} \backslash \triangle\right\} \rightarrow \mathbb{R}^{6 n}$ é continuamente diferenciável, pelo menos num aberto em torno de $(Q(0), \dot{Q}(0)) \in \mathbb{R}^{6 n}$. Tal fato advém da escolha das posições iniciais fora de $\triangle$. São hipóteses suficientes para o Teorema de Existência e Unicidade, vide [42], para garantir que existe $\delta \in \mathbb{R}$ positivo e uma única aplicação

$$
\begin{aligned}
& S:(-\delta, \delta) \rightarrow \mathbb{R}^{6 n} \\
& t \quad \mapsto S(t)=(Q(t), \dot{Q}(t))
\end{aligned}
$$

solução de (3.11). Muito embora tal solução exista e seja única ela não pode ser determinada por quadraturas, pois de acordo com o próximo resultado, de E. Julliard-Toseal em [17], existem apenas 10 integrais primeiras para o problema de $n$ corpos em $\mathbb{R}^{3}$. Em verdade, Julliard-Tosel generalizou os resultados obtidos por H. Bruns em [5]. Abaixo é enunciado o teorema de Bruns generalizado.

Teorema 3.2.1 No problema Newtoniano de $(n+1)$-corpos em $\mathbb{R}^{p}$, com $n \geq 2$ e $1 \leq p \leq n+1$, toda integral primeira que seja algébrica com respeito às posições, momentos e tempo é uma função algébrica das integrais primeiras clássicas: a energia, as $p(p-1) / 2$ componentes do momento angular total e as $2 p$ integrais que vem do movimento retilíneo uniforme do centro de massa.

Para o caso $p=3$, vê-se que o resultado é exatamente 10 quantidades e pelo teorema anterior se $n>2$ não se conhece métodos para determinar diretamente uma solução. O que é feito, geralmente, é estudar casos particulares, fazendo restrições nas massas ou nas próprias configurações ao longo do tempo. No próximo Capítulo será estudado um tipo de solução possível, que é o caso em que as posições iniciais formam configurações centrais.

Na próxima seção são discutidos alguns resultados conhecidos do problema Newtoniano de $n$ corpos geral. 


\subsection{Alguns resultados do Problema de $n$ corpos}

Vamos introduzir aqui alguns conceitos e teoremas que foram obtidos ao longo dos mais de trezentos anos de estudo do problema de $n$ corpos.

Primeiramente, observe que a quantidade vetorial $C$, centro massa do sistema, pelas leis de conservação tem movimento retilíneo e uniforme. Não há perda de generalidade se considerarmos a origem do sistema de coordenadas sobre $C$.

Definição 3.3.1 No problema Newtoniano de $n$ corpos, chamaremos de referencial baricêntrico, um referencial inercial, tal que

$$
C=\sum_{i=1}^{n} m_{i} q_{i}=0,
$$

Assim, considere daqui por diante as definições com relação ao referencial baricêntrico ou referencial do centro de massa.

Definição 3.3.2 No problema Newtoniano de $n$ corpos, no referencial baricêntrico, chamaremos de momento de inércia a seguinte quantidade escalar

$$
I=\frac{1}{2} \sum_{i=1}^{n} m_{i}\left|q_{i}\right|^{2}
$$

que também pode ser escrita como

$$
I=\frac{1}{2 \mu} \sum_{1 \leq i<j \leq n}^{n} m_{i} m_{j}\left|q_{i}-q_{j}\right|^{2}
$$

A quantidade $I$ nos dá uma estimativa do tamanho da configuração, uma vez que em (3.13) vê-se que a máxima separação entre dois corpos é super-estimada por $I$.

Teorema 3.3.3 No problema Newtoniano de $n$ corpos, vale a seguinte igualdade

$$
\ddot{I}=2 T+V=T+E=2 E-V .
$$

Esta é a chamada de identidade Lagrange-Jacobi. 
Prova: Derivando a expressão (3.12) obtemos,

$$
\dot{I}=\frac{1}{2} \sum_{i=1}^{n} m_{i} q_{i} \bullet \dot{q}_{i}
$$

e por conseguinte,

$$
\ddot{I}=\sum_{i=1}^{n} m_{i} \dot{q}_{i}{ }^{2}+\sum_{i=1}^{n} m_{i} q_{i} \bullet \ddot{q}_{i} .
$$

Lembrando que $V$ é uma função homogênea de grau (-1) nas componentes dos $q_{i}$ 's, temos

$$
\sum_{i=1}^{n} q_{i} \bullet \nabla_{i} V=-V
$$

$\log$,

$$
\ddot{I}=2 T+\sum_{i=1}^{n} q_{i} \bullet \nabla_{i} V=2 T+V=T+E=2 E-V .
$$

Um corolário direto do Teorema 3.3.3 diz que não há solução do problema de $n$ corpos na qual os corpos permaneçam em repouso.

Corolário 3.3.4 No problema Newtoniano de n corpos não existe solução de equilíbrio.

Prova: De fato, se existir tal solução de equilíbrio

$$
\dot{I}=0
$$

e

$$
T=0
$$

Donde,

$$
U=0
$$

o que é uma contradição. 
Teorema 3.3.5 Para o problema Newtoniano de $n$ corpos, descrito acima, vale a seguinte desigualdade para a norma do momento angular

$$
J^{2} \leq 4 I(\ddot{I}-E)
$$

Esta é a chamada desigualdade de Sundman.

Prova: Lembremos que

$$
|J|=\left|\sum_{i=1}^{n} m_{i} q_{i} \wedge \dot{q}_{i}\right|,
$$

Logo, pelas desigualdades triangular e de Cauchy-Schwartz

$$
|J| \leq \sum_{i=1}^{n}\left|m_{i} q_{i} \wedge \dot{q}_{i}\right| \leq \sum_{i=1}^{n} m_{i}\left|q_{i}\right|\left|\dot{q}_{i}\right|=\sum_{i=1}^{n} \sqrt{m_{i}}\left|q_{i}\right| \sqrt{m_{i}}\left|\dot{q}_{i}\right|
$$

que ainda podemos escrever como sendo

$$
|J| \leq \sum_{i=1}^{n} \sqrt{m_{i}}\left|q_{i}\right| \sqrt{m_{i}}\left|\dot{q}_{i}\right| \leq \sqrt{\sum_{i=1}^{n} m_{i} q_{i}^{2}} \sqrt{\sum_{i=1}^{n} m_{i} \dot{q}_{i}^{2}}
$$

donde

$$
|J| \leq \sqrt{\sum_{i=1}^{n} m_{i} q_{i}^{2}} \sqrt{\sum_{i=1}^{n} m_{i} \dot{q}_{i}^{2}}=\sqrt{2 I} \sqrt{2 T}=\sqrt{4 I T}
$$

elevando ambos os membros ao quadrado e usando o teorema anterior temos

$$
J^{2} \leq 4 I T=4 I(\ddot{I}-E)
$$

Um corolário importante da desigualdade de Sundman é a obtenção de estimativas para a máxima e mínima separação entre os corpos numa dada configuração.

Corolário 3.3.6 No problema Newtoniano de $n$ corpos existem constantes positivas $a_{1}, a_{2}, a_{3}, a_{4}$, 
que dependem apenas das massas, tais que valem as seguintes

$$
a_{1} \sqrt{I} \leq \max _{1 \leq i<j \leq n}\left|q_{i}-q_{j}\right| \leq a_{2} \sqrt{I}
$$

$e$

$$
-\frac{a_{3}}{V} \leq \min _{1 \leq i<j \leq n}\left|q_{i}-q_{j}\right| \leq-\frac{a_{4}}{V}
$$

A seguir vamos usar estes resultados para demonstrar alguns teoremas acerca do colapso total, que é o caso em que todos os corpos colidem num mesmo instante. Uma exposição direta e bem escrita destes teoremas pode ser encontrada em [46].

Lema 3.3.7 No problema Newtoniano de $n$ corpos, se ocorrer o colapso total então ele ocorre na origem do sistema baricêntrico.

Prova: Seja $t_{c}$ o instante do colapso total, então existem e coincidem os seguintes limites

$$
\lim _{t \rightarrow t_{c}} q_{j}(t)=q_{j}\left(t_{c}\right)=q_{c}, \forall j=1, \ldots, n .
$$

Portanto, com $k \neq j$,

$$
\lim _{t \rightarrow t_{c}}\left|q_{j}(t)-q_{k}(t)\right|=\left|q_{c}-q_{c}\right|=0
$$

Como $0 \leq \min _{1 \leq i<j \leq n}\left|q_{i}-q_{j}\right| \leq\left|q_{i}-q_{k}\right|$, para todo $j \neq k$, segue do Corolário 3.3.6 que

$$
0=\lim _{t \rightarrow t_{c}} I(t)=\frac{1}{2} \sum_{i=1}^{n} m_{i}\left|q_{i}\left(t_{c}\right)\right|^{2},
$$

daí, para todo $i=1, \ldots, n$ vale $q_{i}\left(t_{c}\right)=0$.

Lema 3.3.8 No problema Newtoniano de $n$ corpos, se ocorrer o colapso total então ele ocorre em tempo finito. 
Prova: Observe primeiramente que, como visto na prova do lema anterior, no colapso total $I=0$.

Suponha, por contradição, que o colapso ocorre em $t_{c}=+\infty$. Defina $d_{\min }(t)$ como sendo a menor distância entre os corpos no instante $t$, que é

$$
d_{\min }(t)=\min _{i \neq j}\left|q_{i}(t)-q_{j}(t)\right|
$$

$\log 0$,

$$
\lim _{t \rightarrow+\infty} d_{\min }(t)=0
$$

Portanto, o potencial $V$ no colapso tem limite

$$
\lim _{t \rightarrow+\infty} V(t)=-\infty
$$

Pela identidade de Lagrange-Jacobi vem que,

$$
\lim _{t \rightarrow+\infty} \ddot{I}(t)=2 E-\lim _{t \rightarrow+\infty} V(t)=+\infty .
$$

Assim, existe $t_{1}$ tal que,

$$
t>t_{1} \Rightarrow \ddot{I}(t) \geq 1
$$

Integrando esta desigualdade duas vezes obtemos,

$$
I(t) \geq \frac{1}{2} t^{2}+c_{1} t+c_{2}
$$

onde $c_{1}$ e $c_{2}$ são constantes de integração que dependem apenas de $t_{1}$. Desta forma,

$$
\lim _{t \rightarrow+\infty} I(t)=+\infty
$$

mas isto é uma contradição, já que no colapso $I=0$. O mesmo raciocínio pode ser usado para o caso em que o colapso se dê em $t=-\infty$. 
Teorema 3.3.9 No problema Newtoniano de $n$ corpos, se ocorre o colapso total,

$$
\bar{Q}=\left\{\left(q_{1}, \ldots, q_{n}\right) \in \triangle / q_{1}=q_{2}=\ldots=q_{n}\right\}
$$

então o momento angular total é nulo. Este resultado é conhecido como teorema de SundmanWeierstrass.

Prova: Pelo Lema 3.3.8 podemos considerar que o tempo de colapso é $t_{c}$, com $t_{c}<\infty$. Daí temos

$$
\lim _{t \rightarrow t_{c}} d_{\min }(t)=0
$$

$\log$,

$$
\lim _{t \rightarrow t_{c}} V(t)=-\infty
$$

Usando a igualdade de Lagrange-Jacobi nos vem que

$$
\lim _{t \rightarrow t_{c}} \ddot{I}(t)=+\infty
$$

Como $I(t)$ é regular em $t_{c}$, podemos garantir que numa vizinhança à esquerda de $t_{c}$ vale $\ddot{I}(t)>0$. Por definição, $I(t)$ é uma função não negativa, portanto, podemos concluir que $\dot{I}(t)<0$ nessa vizinhança. Usando agora a desigualdade de Sundman podemos escrever

$$
\ddot{I}(t) \geq \frac{J^{2}}{4 I(t)}+E .
$$

Multiplicando ambos os membros por $-\dot{I}(t)>0$, temos

$$
-\dot{I} \ddot{I} \geq-\frac{J^{2} \dot{I}}{4 I}-E \dot{I}
$$


Daí, integrando, temos

$$
-\frac{1}{2} \frac{d}{d t}\left(\dot{I}^{2}\right) \geq-\frac{J^{2}}{4} \frac{d}{d t} \ln I-E \frac{d}{d t} I
$$

Integrando (3.14), agora em $\left[t_{1}, t_{2}\right]$, com $t_{2}<t_{c}$, vem

$$
-\frac{1}{2}\left[\left(\dot{I}\left(t_{2}\right)\right)^{2}-\left(\dot{I}\left(t_{1}\right)\right)^{2}\right] \geq-\frac{J^{2}}{4} \ln \left(\frac{I\left(t_{2}\right)}{I\left(t_{1}\right)}\right)-E\left[I\left(t_{2}\right)-I\left(t_{1}\right)\right]
$$

que pode ser reescrito como

$$
\frac{J^{2}}{4} \ln \left(\frac{I\left(t_{2}\right)}{I\left(t_{1}\right)}\right) \leq E\left[I\left(t_{2}\right)-I\left(t_{1}\right)\right]+\frac{1}{2}\left[\left(\dot{I}\left(t_{1}\right)\right)^{2}-\left(\dot{I}\left(t_{2}\right)\right)^{2}\right]
$$

Das considerações sobre as derivadas de $I$, feitas acima, temos

$$
\left\{\begin{array}{l}
\left(\dot{I}\left(t_{1}\right)\right)^{2}-\left(\dot{I}\left(t_{2}\right)\right)^{2} \leq\left(\dot{I}\left(t_{1}\right)\right)^{2} \\
I\left(t_{2}\right)-I\left(t_{1}\right) \leq I\left(t_{2}\right) .
\end{array}\right.
$$

Inserindo (3.15) e (3.16) em (3.14) e usando esta última consideração, nos vem

$$
\frac{J^{2}}{4} \leq \frac{E I\left(t_{2}\right)+\left(\dot{I}\left(t_{1}\right)\right)^{2}}{\ln \left(\frac{I\left(t_{1}\right)}{I\left(t_{2}\right)}\right)}
$$

Tomando agora

$$
\lim _{t_{2} \rightarrow t_{c}} \frac{E I\left(t_{2}\right)+\left(\dot{I}\left(t_{1}\right)\right)^{2}}{\ln \left(\frac{I\left(t_{1}\right)}{I\left(t_{2}\right)}\right)}=0
$$

daí temos que no caso de colisão total

$$
J^{2}=0
$$

O Teorema de Sundman-Weierstrass tem uma interpretação interessante no contexto da "estabilidade" do problema Newtoniano de $n$ corpos. Note que ele dá uma condição necessária para que o sistema colapse sobre si mesmo. No próximo Capítulo veremos uma condição, adicional, sobre a 
configuração inicial que, junto com momento angular total nulo, levam certamente à colisão total. Embora, não se saiba se tal situação é a única que leva ao colapso total.

A estabilidade do movimento dos corpos celestes é uma questão chave da Mecânica Celeste. Porém, uma das dificuldades inerentes ao problema da estabilidade no problema Newtoniano de $n$ corpos é, como definir "estabilidade". Comumente dizemos que uma solução é estável se

1. O intervalo maximal onde a solução está definida é $\mathbb{R}$;

2. A solução não experimenta colisões, $\left|q_{i}(t)-q_{j}(t)\right| \neq 0, \forall t \in \mathbb{R}$;

3. A solução é limitada, i.e., $\exists K \in \mathbb{R}_{+}$, tal que, $\max _{i \neq j}\left|q_{i}(t)-q_{j}(t)\right| \leq K, \forall t \in \mathbb{R}$;

O teorema seguinte fornece um resultado importante que é um critério para não estabilidade, no sentido que dá uma condição para o movimento ser ilimitado.

Teorema 3.3.10 No problema Newtoniano de $n$ corpos, se a energia mecânica total do sistema é positiva, então o movimento é ilimitado. Este resultado é conhecido como critério de Jacobi.

Prova: Recorde que da definição de estabilidade o intervalo de definição da solução é $\mathbb{R}$. Suponha

$$
E=T+V>0
$$

assim, identidade de Lagrange-Jacobi,

$$
\ddot{I}=T+E \geq E>0 .
$$

Integrando esta desigualdade temos

$$
I(t) \geq \frac{E}{2} t^{2}+c_{1} t+c_{2},
$$

No limite $t \rightarrow+\infty$, temos uma contradição com a definição. 
Assim, uma condição necessária para a estabilidade do movimento é a energia mecânica total do sistema ser negativa, uma vez que o caso da energia nula é analogamente levado a movimento ilimitado.

No próximo Capítulo veremos algumas soluções particulares e a relação que existe entre estas soluções e as configurações centrais. 


\section{Capítulo 4}

\section{Soluções Particulares do Problema de $n$ Corpos}

Conforme foi visto no Capítulo precedente, o Problema Newtoniano de $n$ corpos não pode ser integrado diretamente por quadraturas. Tal fato, torna o estudo de soluções particulares de grande valor. Neste Capítulo introduziremos o conceito de soluções homográficas e derivaremos as relações que uma configuração inicial deve ter para existir a possibilidade de tais soluções. Considere, neste Capítulo, todas definições com relação ao referencial baricêntrico. Os resultados deste Capítulo podem ser encontrados em [31].

\subsection{Soluções Homotéticas}

Definição 4.1.1 No problema Newtoniano de n corpos, chamaremos uma solução de homotética se existe uma função positiva, $r(t)>0$, tal que a dinâmica dos corpos é dada por

$$
Q(t)=r(t) Q_{0}
$$

com $Q_{0} \in X=\mathbb{R}^{3 n} \backslash \Delta$ e para todo t no intervalo maximal de solução.

Note que a configuração $Q_{0}$ é preservada neste tipo de solução. Se $r \rightarrow 0$ teremos uma solução do tipo colisão total, da qual já vimos algumas propriedades.

Admitamos que existam soluções deste tipo e que $r(t)$ seja suficientemente diferenciável para tal. Vamos levar a expressão (4.1) nas equações de movimento (3.10) e ver que propriedades devem ter $Q_{0}$ e $r$. Assim temos,

$$
M \frac{d^{2}}{d t^{2}}\left(r(t) Q_{0}\right)=-\nabla V\left(r(t) Q_{0}\right)
$$


ou ainda,

$$
\ddot{r}(t) M Q_{0}=-\frac{1}{r^{2}(t)} \nabla V\left(Q_{0}\right) .
$$

Multiplicando ambos os lados por $Q_{0}^{t}$ e usando a homogeneidade do potencial, recebemos

$$
\ddot{r}=\frac{\lambda}{r^{2}},
$$

onde,

$$
\lambda=\frac{V\left(Q_{0}\right)}{Q_{0}^{t} M Q_{0}}=\frac{V\left(Q_{0}\right)}{2 I\left(Q_{0}\right)}<0 .
$$

Note que a expressão (4.3) é a equação de Kepler para o caso com momento angular nulo, conforme visto no Capítulo 2 tal condição leva às chamadas soluções de colisão ejeção. Desta forma $r(t)$ deve ser de classe $C^{2}$ e satisfazer à equação de Kepler.

Vamos voltar à equação de movimento e ver que relação deve satisfazer a condição inicial. Uma vez que, $\lambda=\lambda\left(Q_{0}\right)$ temos

$$
\lambda M Q_{0}=-\nabla V\left(Q_{0}\right)
$$

Assim, para construir soluções homotéticas, primeiro é necessário resolver a equação (4.4) e em seguida multiplicar por uma solução colinear da equação de Kepler. Ajustando as velocidades podemos construir soluções de colisão total, de ejeção-colisão ou ejeção total, na qual todos os corpos se afastam assintoticamente.

Definição 4.1.2 No problema Newtoniano de $n$ corpos, chamaremos de Configuração Central uma solução da equação

$$
\lambda M Q=-\nabla V(Q) .
$$

Esta equação será chamada de equação das configurações centrais.

Note a dificuldade algébrica na solução desta equação, que na realidade é um sistema de $n$ equações algébricas nas posições. Muito pouco se sabe sobre as soluções deste sistema. Adiante, comentaremos alguns resultados conhecidos e as questões envolvendo a equação de configurações centrais. 
Vamos resolver um caso simples da equação (4.4), quando $n=3$ e com massas sobre uma mesma reta. Este caso é conhecido na literatura como configurações de Euler [7].

Exemplo 4.1.3 Considere um caso colinear de três corpos de massas, $m_{1}, m_{2}, m_{3}$ com posições na reta real, tais que $q_{1}<q_{2}<q_{3}$, tomando $x=q_{2}-q_{1}$ e $y=q_{3}-q_{1}$. Vide figura 4.1.

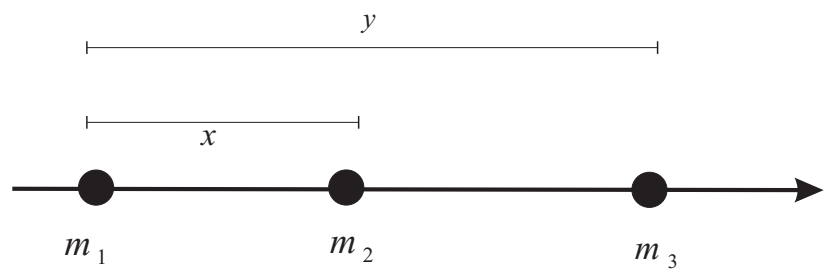

Figura 4.1: Configuração colinear de Euler.

A equação de configurações centrais neste caso se reduz a

$$
\begin{aligned}
& \lambda x=-\frac{m_{1}+m_{2}}{x^{2}}-\frac{m_{3}}{y^{2}}+\frac{m_{3}}{(y-x)^{2}}, \\
& \lambda y=-\frac{m_{1}+m_{3}}{y^{2}}-\frac{m_{2}}{x^{2}}+\frac{m_{2}}{(y-x)^{2}} .
\end{aligned}
$$

Isolando $\lambda$ nas equações de (4.5) e inserindo a variável $z=y / x$ temos a seguinte equação polinomial,

$f(z)=\left(m_{1}+m_{3}\right) z^{5}+\left(3 m_{1}+2 m_{2}\right) z^{4}+\left(3 m_{1}+m_{2}\right) z^{3}-\left(3 m_{3}+m_{2}\right) z^{2}-\left(3 m_{3}+2 m_{2}\right) z-\left(m_{3}+m_{2}\right)=0$.

É fácil ver, usando a regra dos sinais de Descartes, que esta equação tem uma única raiz positiva. E que encontrada esta raiz, basta voltar às equações que teremos um família a 1 parâmetro de soluções. Uma pergunta natural é: Quando mudarmos este parâmetro a solução da equação (4.5) muda? A resposta claramente é sim, porém mais adiante tomaremos como relação de equivalência dilatações e contrações de $X$ centrada no centro de massa do sistema. Uma vez que o próximo resultado nos mostra que, se uma configuração $Q$ é solução de da equação de configurações centrais, então $k Q$ também é solução de uma equação do mesmo tipo.

Teorema 4.1.4 Seja $Q$ uma solução da equação de configurações centrais, com constante $\lambda$. 
Então, para toda constante $k \in \mathbb{R}_{+}, k Q$ é solução da equação de configuração central com constante $\tilde{\lambda}=\lambda / k^{3}$.

Prova: Por cálculo direto vemos que

$$
-\nabla V(k Q)=-\frac{1}{k^{2}} \nabla V(Q)
$$

Por outro lado, como $Q$ é solução da equação de configurações centrais com $\lambda$, temos

$$
\lambda M Q=-\nabla V(Q) .
$$

Inserindo a equação (4.7) na equação (4.6) teremos,

$$
-\nabla V(k Q)=-\frac{1}{k^{2}} \lambda M Q=-\frac{1}{k^{3}} \lambda M(k Q)
$$

que é o resultado desejado.

Na próxima seção tratamos um outro tipo de solução, particularmente interessante que são os equilíbrios relativos, este tipo de solução foi tratada primeiramente por Lagrange, no caso de três corpos, em [18].

\subsection{Soluções de Equilíbrio Relativo}

Definição 4.2.1 No problema Newtoniano de $n$ corpos, chamaremos uma solução de equilíbrio relativo se existe uma aplicação no grupo de transformações rígidas de $\mathbb{R}^{3}, g(t) \in \operatorname{Eucl}\left(\mathbb{R}^{3}\right)$, tal que a dinâmica dos corpos é dada por

$$
Q(t)=g(t) Q_{0}
$$


com $Q_{0} \in X=\mathbb{R}^{3 n} \backslash \Delta$ e para todo t no intervalo maximal de solução. Aqui, entendemos a ação do grupo Eucl $\left(\mathbb{R}^{3}\right)$ sobre $\mathbb{R}^{3 n}$ como a ação sobre cada corpo em $\mathbb{R}^{3}$,

$$
g(t) Q_{0}=\left(g(t) q_{01}, \ldots, g(t) q_{0 n}\right)^{t} .
$$

Como tratamos o caso com referencial baricêntrico é suficiente considerar $g(t)=A(t) \in S O(3)$. Assim, a equação (4.8) se escreve como

$$
Q(t)=A(t) Q_{0}
$$

Uma configuração $Q_{0}$ para a qual é possível obter este tipo de solução será chamada de Equilíbrio Relativo.

Novamente, admitindo que seja possível este tipo de solução, vejamos nas equações de movimento que tipo relação devem satisfazer $Q_{0}$ e $A(t)$. Assim, temos

$$
\ddot{A} M Q_{0}=-A \nabla V\left(Q_{0}\right)
$$

ou ainda,

$$
A^{-1} \ddot{A} M Q_{0}=-\nabla V\left(Q_{0}\right)
$$

Lema 4.2.2 Se $\Omega(t) \in S O(3)$, então $\Omega^{-1} \dot{\Omega} \in \mathcal{A}(3)$, isto é, $\Omega^{-1} \dot{\Omega}$ uma matriz anti-simétrica.

Prova: De fato, $\Omega^{t} \Omega=I d=\Omega^{-1} \Omega$, com $I d$ matriz identidade em $\mathbb{R}^{3}$, derivando esta relação obtemos

$$
\frac{d}{d t} \Omega^{t} \dot{\Omega}=0
$$

donde,

$$
\dot{\Omega}^{t} \Omega+\Omega^{t} \dot{\Omega}=0 .
$$

Mas, isto implica que, $\Omega^{t} \dot{\Omega} \in \mathcal{A}\left(\mathbb{R}^{3}\right)$. 
Seja $W(t)=A^{-1} \dot{A}$, pelo Lema 4.2.2, $W(t)$ é uma matriz anti-simétrica. Como existe um isomorfismo entre $\mathcal{A}\left(\mathbb{R}^{3}\right)$ e $\mathbb{R}^{3}$, podemos associar a $W$ um vetor, $w=\left(w_{1}, w_{2}, w_{3}\right) \in \mathbb{R}^{3}$, tal que

$$
W=\left(\begin{array}{ccc}
0 & -w_{3} & w_{2} \\
w_{3} & 0 & -w_{1} \\
-w_{2} & w_{1} & 0
\end{array}\right)
$$

Derivando a expressão de $W$ obtemos,

$$
\dot{W}=A^{-1} \ddot{A}-\left(A^{-1} \dot{A} A^{-1}\right) \dot{A}=A^{-1} \ddot{A}-W^{2} .
$$

Assim,

$$
A^{-1} \ddot{A}=\dot{W}+W^{2},
$$

ou mais explicitamente,

$$
A^{-1} \ddot{A}=\left(\begin{array}{ccc}
-\left(w_{2}^{2}+w_{3}^{2}\right) & -\dot{w}_{3}+w_{1} w_{2} & \dot{w}_{2}+w_{1} w_{3} \\
\dot{w}_{3}+w_{1} w_{2} & -\left(w_{1}^{2}+w_{3}^{2}\right) & -\dot{w}_{1}+w_{2} w_{3} \\
-\dot{w}_{2}+w_{1} w_{2} & \dot{w}_{1}+w_{2} w_{3} & -\left(w_{1}^{2}+w_{2}^{2}\right)
\end{array}\right)
$$

Note que pela expressão (4.9), temos as seguintes equações

$$
A^{-1} \ddot{A} q_{0 i}=-m_{i}^{-1} \nabla_{i} V\left(Q_{0}\right)=\text { constante }, \quad i=1, \ldots, n
$$

Vamos usar esta última expressão para mostrar que $A^{-1} \ddot{A}$ é uma matriz constante, pois assim como $A^{-1} \ddot{A}=\dot{W}+W^{2}$ é uma decomposição nas partes simétricas e anti-simétrica, teremos $W^{2}(t)$ também constante. Em decorrência disto, temos que $A$ é uma matriz de rotação uniforme em torno de um eixo fixo.

Como temos na equação (4.10) uma matriz, que em princípio depende do tempo, aplicada a $n$ vetores e dando como resultado outros $n$ vetores independentes do tempo. Temos três possíveis casos: 
- Os vetores $q_{01}, \ldots, q_{0 n}$ geram $\mathbb{R}^{3}$, neste caso $A^{-1} \ddot{A}$ necessariamente é constante;

- O vetores $q_{01}, \ldots, q_{0 n}$ geram um subespaço afim de dimensão 2 , assim podemos escolher uma base de modo que $A^{-1} \ddot{A}$ seja constante nas duas primeiras colunas e ainda escrever na seguinte forma,

$$
A^{-1} \ddot{A}=\left(\begin{array}{ccc}
c_{11} & c_{12} & s_{1}(t) \\
c_{21} & c_{22} & s_{2}(t) \\
0 & 0 & s_{3}(t)
\end{array}\right) \text {. }
$$

Utilizando a escrita explícita de $A^{-1} \ddot{A}$ teremos as seguintes igualdades

$$
\left\{\begin{array}{l}
w_{2}^{2}+w_{3}^{2}=-c_{11} \\
w_{1}^{2}+w_{3}^{2}=-c_{22} \\
-\dot{w}_{3}+w_{1} w_{2}=c_{12} \\
\dot{w}_{3}+w_{1} w_{2}=c_{21} \\
-\dot{w}_{2}+w_{1} w_{3}=0 \\
\dot{w}_{1}+w_{2} w_{3}=0
\end{array}\right.
$$

As 4 primeiras equações de (4.11) implicam que

$$
\left\{\begin{array}{l}
w_{1}^{2}-w_{2}^{2}=c_{11}-c_{22} \\
w_{1} w_{2}=\frac{c_{12}+c_{21}}{2}
\end{array}\right.
$$

Donde $w_{1}$ e $w_{2}$ são constantes e portanto, $s_{3}(t)$ é constante. Mas, inserindo esta informação nas duas últimas equações de (4.11) teremos $w_{1} w_{3}=w_{2} w_{3}=0$, $\operatorname{logo} s_{1}(t)=s_{2}(t)=0$.

Segue que,

$$
A^{-1} \ddot{A}=\left(\begin{array}{ccc}
c_{11} & c_{12} & 0 \\
c_{21} & c_{22} & 0 \\
0 & 0 & c_{33}
\end{array}\right) \text {. }
$$

como queríamos. 
- Por fim, no caso em que todas as massas são colineares, para que após a rotação as massas continuem colineares é necessário que as velocidades sejam todas co-planares à reta que contém as massas. Assim, pela conservação do momento angular total, o movimento deve ser sempre planar. Segue que existe uma base na qual o vetor $w$, que define a matriz $W$, é da forma $w=\left(0,0, w_{3}\right)$. Daí

$$
A^{-1} \ddot{A}=\left(\begin{array}{ccc}
-w_{3}^{2} & -\dot{w}_{3} & 0 \\
\dot{w}_{3} & -w_{3}^{2} & 0 \\
0 & 0 & 0
\end{array}\right)
$$

Mas, no caso dos corpos colineares, $A^{-1} \ddot{A}$ deve levar corpos de uma reta em outra. Daí, necessariamente, devemos ter $\dot{w}_{3}=0$, o que implica $W$ constante, como queríamos.

Como visto, no itens acima o vetor $w$, que define a matriz $W$ deve ser constante. Logo, sem perda de generalidade, consideremos uma base na qual $w=(0,0, k)$. Daí,

$$
A^{-1} \ddot{A}=\left(\begin{array}{ccc}
-k^{2} & 0 & 0 \\
0 & -k^{2} & 0 \\
0 & 0 & 0
\end{array}\right) \text {. }
$$

Voltando na equação (4.9), com esta matriz, teremos equações da forma

$$
A^{-1} \ddot{A} m_{i} q_{0 i}=-\nabla_{i} V\left(Q_{0}\right)
$$

Note que isto implica que todas as interações sobre os corpos são co-planares, tal fato implica que os corpos também devem ser co-planares.

Inserindo esta expressão de $A^{-1} \ddot{A}$ explicitamente na equação (4.9), teremos que a configuração $Q_{0}$ deve respeitar afim de termos solução de equilíbrio relativo é a seguinte,

$$
\lambda M Q_{0}=-\nabla V\left(Q_{0}\right)
$$

onde $\lambda=-k^{2}$. 
É notável a semelhança entre a equação (4.4) e a equação (4.12). De fato, afora o fato de que a última configuração deve ser planar temos a mesma equação.

Assim, para construir soluções de equilíbrio relativo primeiro é necessário resolver a equação (4.12) e em seguida multiplicar por uma matriz de rotação uniforme em torno de um eixo passando pelo centro de massa do sistema. No exemplo 4.1.3, calculamos uma configuração central colinear,

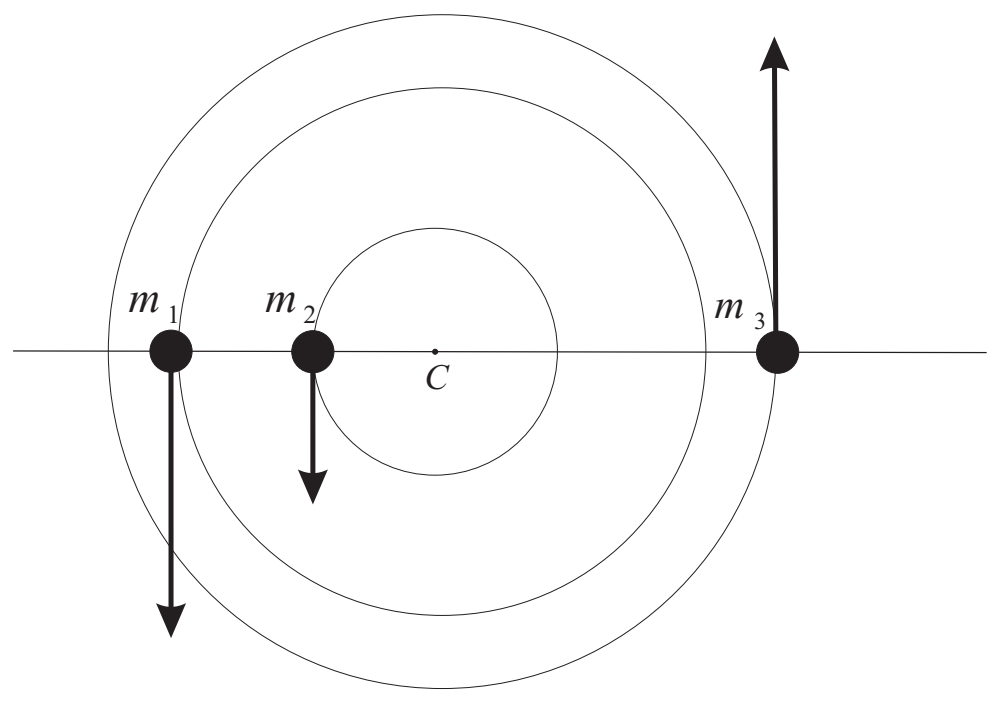

Figura 4.2: Solução de equilíbrio relativo de Euler.

que em particular é planar. Se multiplicarmos esta configuração por uma matriz de rotação uniforme em torno de um eixo passando pelo centro de massa teremos um movimento como mostrado na figura 4.2, que é a solução de equilíbrio relativo de Euler, vide [7].

Outro exemplo clássico de movimento de equilíbrio relativo com três massas foi encontrado por Lagrange, em [18]. No Capítulo seguinte, quando enunciarmos o teorema do perpendicular bissetor, mostraremos que três massas nos vértices de um triângulo equilátero formam uma configuração central que independe dos valores das massas. Considerando, pois, tal configuração teremos um movimento como mostrado na figura 4.3, que são as órbitas periódicas de Lagrange.

Na próxima seção combinaremos as duas últimas soluções vistas, isto é veremos soluções particulares onde a dinâmica das massas é dada por rotações e homotetias simultaneamente. Estas soluções são chamadas de homográficas, uma boa referência para este estudo é o trabalho de C. Vidal e G. Remildo, em [45]. 


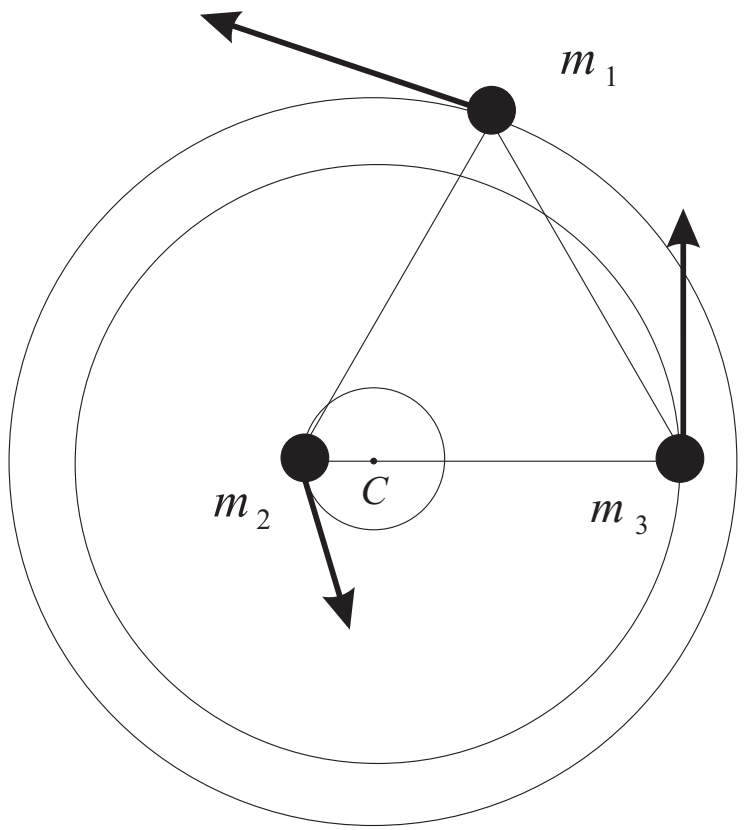

Figura 4.3: Solução de equilíbrio relativo de Lagrange.

\subsection{Soluções Homográficas}

Definição 4.3.1 No problema Newtoniano de $n$ corpos, chamaremos uma solução de homográfica se existem uma aplicação no grupo de transformações rígidas de $\mathbb{R}^{3}, g(t) \in \operatorname{Eucl}\left(\mathbb{R}^{3}\right)$ e uma função positiva $r(t)$, tais que a dinâmica dos corpos é dada por

$$
Q(t)=r(t) g(t) Q_{0},
$$

com $Q_{0} \in X=\mathbb{R}^{3 n} \backslash \Delta$ e para todo $t$ no intervalo maximal de solução. Aqui, entendemos, novamente, a ação do grupo Eucl $\left(\mathbb{R}^{3}\right)$ sobre $\mathbb{R}^{3 n}$ como a ação sobre cada corpo em $\mathbb{R}^{3}$,

$$
g(t) Q_{0}=\left(g(t) q_{01}, \ldots, g(t) q_{0 n}\right)^{t} .
$$


Novamente, como tratamos o caso com referencial baricêntrico é suficiente considerar $g(t)=$ $A(t) \in S O(3)$. Assim, a equação (4.13) se escreve como

$$
Q(t)=r(t) A(t) Q_{0}
$$

Novamente, admitindo que seja possível este tipo de solução, vejamos nas equações de movimento que tipo relação devem satisfazer $Q_{0}, r(t)$ e $A(t)$. Assim temos,

$$
\ddot{r A M} Q_{0}=-\frac{A}{r^{2}} \nabla V\left(Q_{0}\right)
$$

ou ainda,

$$
r^{2}\left(\ddot{r} I d+2 \dot{r} W+r A^{-1} \ddot{A}\right) M Q_{0}=-\nabla V\left(Q_{0}\right)
$$

onde, $W=A^{-1} \dot{A}$.

Quando tivermos uma configuração central planar, fazendo $r(t)=1$, temos as soluções de equilíbrio relativo. Quando tivermos uma configuração central, fazendo $A=I d$ teremos uma solução homotética.

Como vimos do Lema 4.2.2, $W$ é uma matriz anti-simétrica e novamente associaremos a ela um vetor $w=\left(w_{1}, w_{2}, w_{3}\right)$, desta feita escreveremos a aplicação do lado esquerdo de (4.14) da seguinte forma

$$
B(t)=r^{2}\left(\begin{array}{ccc}
\ddot{r}-r\left(w_{2}^{2}+w_{3}^{2}\right) & -r \dot{w}_{3}-2 \dot{r} w_{3}+r w_{1} w_{2} & r \dot{w}_{2}+2 \dot{r} w_{2}+r w_{1} w_{3} \\
r \dot{w}_{3}+2 \dot{r} w_{3}+r w_{1} w_{2} & \ddot{r}-r\left(w_{1}^{2}+w_{3}^{2}\right) & -r \dot{w}_{1}-2 \dot{r} w_{1}+r w_{2} w_{3} \\
-r \dot{w}_{2}-2 \dot{r} w_{2}+r w_{1} w_{3} & r \dot{w}_{1}+2 \dot{r} w_{1}+r w_{2} w_{3} & \ddot{r}-r\left(w_{1}^{2}+w_{2}^{2}\right)
\end{array}\right) .
$$

Note, que mais uma vez na equação (4.14), temos uma matriz, que depende do tempo, aplicada a $n$ vetores constantes dando como resultado outros $n$ vetores constantes. Novamente mostraremos que as entradas da matriz $W$ devem ser constantes. E assim, $A$ será uma matriz de rotação uniforme, como na seção anterior.

Assuma, por enquanto, que os corpos formem uma configuração não colinear. 
Suponha que $Q_{0}$ gere um espaço afim de dimensão dois ou três. Nesta situação, $B$ pode ser escrito como

$$
B=\left(\begin{array}{ccc}
c_{11} & c_{12} & s_{13}(t) \\
c_{21} & c_{22} & s_{23}(t) \\
s_{31}(t) & s_{32}(t) & s_{33}(t)
\end{array}\right)
$$

Utilizando a escrita explícita de $B$, como na equação (4.15), teremos as seguintes equações,

$$
\left\{\begin{array}{l}
c_{11}=r^{2}\left(\ddot{r}-r\left(w_{2}^{2}+w_{3}^{2}\right)\right) \\
c_{22}=r^{2}\left(\ddot{r}-r\left(w_{1}^{2}+w_{3}^{2}\right)\right) \\
c_{12}=r^{2}\left(-r \dot{w}_{3}-2 \dot{r} w_{3}+r w_{1} w_{2}\right) \\
c_{21}=r^{2}\left(r \dot{w}_{3}+2 \dot{r} w_{3}+r w_{1} w_{2}\right) .
\end{array}\right.
$$

Combinando estas equações obtemos,

$$
\left\{\begin{array}{l}
c_{11}-c_{22}=r^{3}\left(w_{1}^{2}-w_{2}^{2}\right) \\
c_{12}+c_{21}=2 r^{3} w_{1} w_{2}
\end{array}\right.
$$

Donde, resulta que $r^{3} w_{1}^{2}$ e $r^{3} w_{2}^{2}$ são constantes. Podemos tomar, sem perda de generalidade, uma base na qual $w\left(t_{1}\right)=\left(0,0, w_{3}\left(t_{1}\right)\right)$, onde $t_{1}$ é um instante de tempo escolhido arbitrariamente. Desta forma, $r^{3}(t) w_{1}^{2}(t)=0$ e $r^{3}(t) w_{2}^{2}(t)=0$, sempre. Logo, daqui por diante, podemos incluir o caso em que $Q_{0}$ é uma configuração colinear, pois como visto na seção anterior, neste caso $w$ é da forma $\left(0,0, w_{3}\right)$ e seguem as mesmas conclusões.

Levando este resultado nas entradas da matriz $B$, vemos que $s_{13}=0, s_{23}=0, s_{31}=0$ e $s_{32}=0$. Logo, teremos a matriz $B$ na seguinte forma,

$$
B(t)=r^{2}\left(\begin{array}{ccc}
\ddot{r}-r w_{3}^{2} & -r \dot{w}_{3}-2 \dot{r} w_{3} & 0 \\
r \dot{w}_{3}+2 \dot{r} w_{3} & \ddot{r}-r w_{3}^{2} & 0 \\
0 & 0 & \ddot{r}
\end{array}\right)
$$


No caso em que, $Q_{0}$ gera $\mathbb{R}^{3}$, temos todas as entradas de $B$ constantes. Em particular $r^{2} \ddot{r}$ constante. Isto implica, observando os outros termos da diagonal, que $r^{3} w_{3}^{2}$ é constante. Derivando esta relação, obtemos

$$
3 w_{3} \dot{r}+2 r \dot{w}_{3}=0
$$

aplicando este resultado nos termos fora da diagonal, temos

$$
w_{3} \dot{r}=0 .
$$

Se $\dot{r}$ for nulo teríamos uma solução de equilíbrio relativo. Por outro lado, se $w_{3}$ é nulo teríamos uma solução homotética. Estas soluções não nos interessam aqui. Consideraremos, portanto soluções planares. Assim o vetor $w$ tem componentes $\left(0,0, w_{3}(t)\right)$ numa base cujo plano $x y$ é paralelo ao plano de movimento. Desta forma, a matriz de rotação $A$ se escreve como,

$$
A(t)=\left(\begin{array}{ccc}
\cos \theta & -\operatorname{sen} \theta & 0 \\
\operatorname{sen} \theta & \cos \theta & 0 \\
0 & 0 & 1
\end{array}\right)
$$

onde, $\dot{\theta}(t)=w_{3}(t)$. Com esta nova notação a matriz $B$ é da forma

$$
B(t)=r^{2}\left(\begin{array}{ccc}
\ddot{r}-r \dot{\theta}^{2} & -r \ddot{\theta}-2 \dot{r} \dot{\theta} & 0 \\
r \ddot{\theta}+2 \dot{r} \dot{\theta} & \ddot{r}-r \dot{\theta}^{2} & 0 \\
0 & 0 & \ddot{r}
\end{array}\right) .
$$

Vamos estudar o que acontece com os termos fora da diagonal em $B$. Para isto considere as velocidades em tal solução. É fácil ver que se escrevem como

$$
\dot{q}_{0 i}=A\left(\dot{r} q_{0 i}+r w \wedge q_{0 i}\right)
$$


Inserindo a expressão (4.16) na expressão do momento angular total do sistema, obtemos

$$
J=\sum_{i=1}^{n} m_{i}\left(r A q_{0 i}\right) \wedge A\left(\dot{r} q_{0 i}+r w \wedge q_{0 i}\right)
$$

que ainda podemos escrever como,

$$
J=A r^{2} \sum_{i=1}^{n} m_{i}\left(q_{0 i} \wedge w \wedge q_{0 i}\right)
$$

cuja terceira componente é constante e igual a

$$
J_{3}=r^{2} \dot{\theta}\left(\sum_{i=1}^{n} m_{i}\left(\left|q_{0 i}\right|^{2}\right)\right)
$$

Como estamos com configurações em $X$ a soma acima nunca é nula, logo, $r^{2} \dot{\theta}$ é constante. Note que os termos fora da diagonal em $B$ são, a menos do sinal, a derivada temporal de $r^{2} \dot{\theta}$. Desta forma, teremos que $B$ se escreve como

$$
B(t)=r^{2}\left(\begin{array}{ccc}
\ddot{r}-r \dot{\theta}^{2} & 0 & 0 \\
0 & \ddot{r}-r \dot{\theta}^{2} & 0 \\
0 & 0 & \ddot{r}
\end{array}\right)
$$

Tanto no caso em que $Q_{0}$ gera um espaço afim bidimensional, quanto no caso colinear teremos, a seguinte igualdade,

$$
B(t)=\left(\begin{array}{ccc}
r^{2} \ddot{r}-r^{3} \dot{\theta}^{2} & 0 & 0 \\
0 & r^{2} \ddot{r}-r^{3} \dot{\theta}^{2} & 0 \\
0 & 0 & r^{2} \ddot{r}
\end{array}\right)=\left(\begin{array}{ccc}
\lambda & 0 & 0 \\
0 & \lambda & 0 \\
0 & 0 & h_{3}(t)
\end{array}\right)
$$

As equações,

$$
\left\{\begin{array}{l}
r^{2} \dot{\theta}=c_{1} \\
\ddot{r}-r \dot{\theta}^{2}=\frac{\lambda}{r^{2}}
\end{array}\right.
$$


são a versão polar das equações de Kepler, quando o momento angular é não nulo, conforme visto no Capítulo 2.

Inserindo a expressão (4.17) nas equações de movimento em (4.14), teremos

$$
\lambda M Q_{0}=-\nabla V\left(Q_{0}\right)
$$

Novamente, chamamos a atenção, para a semelhança entre as equações (4.4), (4.12) e agora (4.18). A menos do fato que na primeira a configuração, poderia ser espacial, temos a mesma equação, nos três casos.

Logo, para construir soluções homográficas do problema Newtoniano de $n$ corpos, devemos encontrar uma configuração resolvendo a equação (4.18). Em seguida multiplicar por uma função positiva $r$ e aplicar uma matriz de rotação uniforme $A$, mas de forma que $r$ e a velocidade angular, $\dot{\theta}$, da matriz de rotação respeitem a equação de Kepler, no caso polar.

Vejamos dois exemplos de movimento homográfico.

Considere a configuração colinear de Euler, calculada no exemplo 4.1.3. O movimento será como dado na figura 4.4.

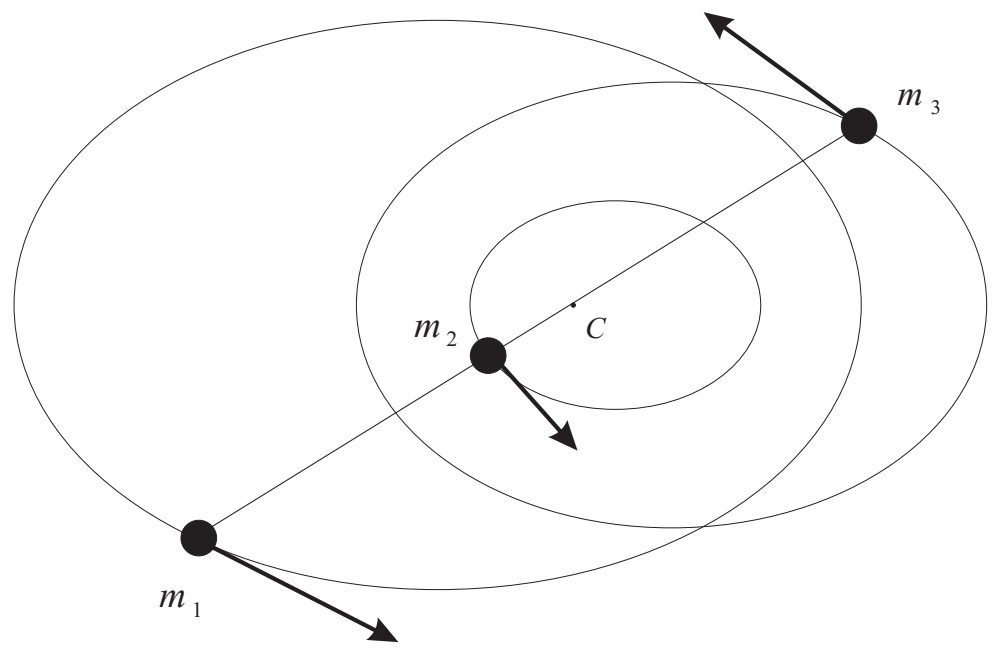

Figura 4.4: Solução homográfica de Euler.

Considerando a configuração equilátera de Lagrange, a qual será calculada no próximo Capítulo, termos um movimento como dado na figura 4.5. 


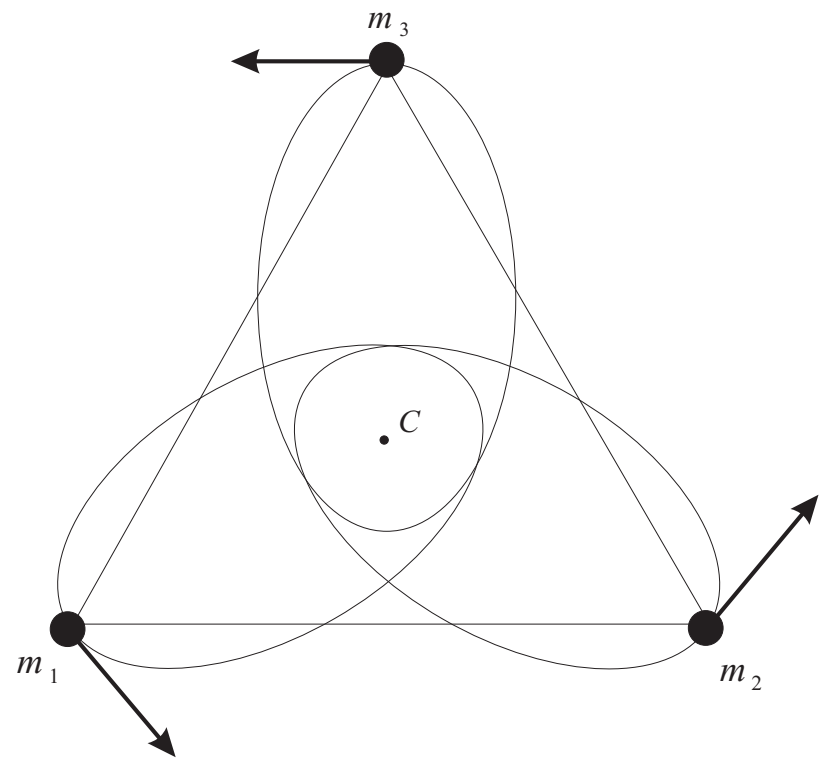

Figura 4.5: Solução homográfica de Lagrange.

Para vermos um exemplo com mais que três massas. Considere um polígono regular com massas iguais em seus vértices. Não é difícil ver que tal configuração respeita as equações na forma de (4.18). Assumindo que este tipo de configuração respeite a equação teremos o movimento como dado na figura 4.6.

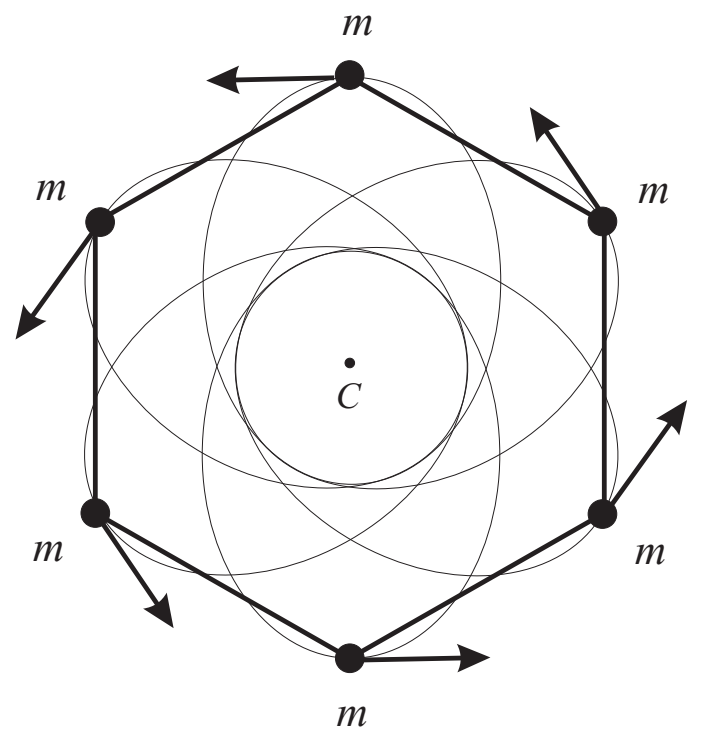

Figura 4.6: Solução homográfica poligonal. 


\subsection{Pontos Críticos da Superfície Energia-Momento}

No Capítulo 3, vimos nas definições do problema Newtoniano de $n$ corpos, que temos dez integrais de movimento neste problema, as quais seguem:

- Uma quantidade da conservação da energia,

$$
E=T+V=E_{0}
$$

- Três quantidades da conservação do momento angular total,

$$
J=J_{0}
$$

- Três quantidades da conservação do momento linear total,

$$
P=P_{0}
$$

- Nesta última vemos que o vetor do centro de massa é escrito da seguinte maneira,

$$
C=C_{0}+t \frac{P_{0}}{\mu}
$$

com $C_{0}$ constante, donde temos mais três quantidades.

Nesta seção desejamos verificar condições para que, dados dez números reais, a imagem inversa das quantidades conservadas, no espaço de fase, seja uma superfície regular de dimensão $6 n-10$.

Para isto, vamos definir a seguinte aplicação.

Definição 4.4.1 No problema Newtoniano de $n$ corpos, chamaremos de Aplicação energia momento, $\mathcal{G}: T X \rightarrow \mathbb{R}^{10}$, dada pela seguinte relação

$$
\mathcal{G}(q, v)=(C(q, v), P(q, v), J(q, v), E(q, v))
$$


onde $q \in X$ e $v \in \mathbb{R}^{3 n}$, como definido no Capítulo anterior.

Como $C$ e $P$ são combinações lineares de coordenas e posições, respectivamente, sempre serão valores regulares quaisquer seis números reais escolhidos. Considere então, $C=0$ e $P=0$, para continuarmos no referencial baricêntrico. Assim consideraremos a aplicação energia-momento baricêntrica

$$
\mathcal{G}_{0}(q, v)=(C=0, P=0, J(q, v), E(q, v)) .
$$

É de se esperar que, para a maioria das escolhas de $(J, E) \in \mathbb{R}^{4}$, o conjunto

$$
\tilde{\mathcal{G}}=\mathcal{G}^{-1}(0,0, J, E)
$$

seja uma variedade de dimensão $6 n-10$.

Consideraremos os elementos de $T X$, na seguinte forma,

$$
(q, v)=\left(q_{1}, \ldots, q_{n}, v_{1}, \ldots, v_{n}\right)^{t}
$$

onde, $q_{i}=\left(x_{i}, y_{i}, z_{i}\right)$ e $v_{i}=\left(\eta_{i}, \xi_{i}, \psi_{i}\right)$.

Considerando $T X$ com métrica usual, induzida de $\mathbb{R}^{6 n}$, vamos definir o seguinte operador gradiente

$$
\tilde{\nabla}=\left(\nabla_{q_{1}}, \ldots, \nabla_{q_{n}}, \nabla_{v_{1}}, \ldots, \nabla_{v_{n}}\right)^{t}
$$

onde, $\nabla_{q_{i}}$ é o gradiente nas coordenadas de posição do corpo de massa $m_{i}$ e $\nabla_{v_{i}}$ é o gradiente nas coordenadas de velocidade deste mesmo corpo. Queremos localizar os pontos onde $\tilde{\nabla} \mathcal{G}_{0}$ não tem posto 10. Vamos calcular, separadamente, cada gradiente das aplicações envolvidas na definição de $\mathcal{G}_{0}$. Assim temos,

- Para o centro de massa do sistema

$$
\tilde{\nabla} C=\frac{1}{\mu}\left(m_{1} I d, \ldots, m_{n} I d, 0 I d, \ldots, 0 I d\right)^{t}
$$


que é uma matriz $6 n \times 3$ em cada ponto, aqui $0 I d$ representa a matriz nula $3 \times 3$.

- Para o momento linear total do sistema

$$
\tilde{\nabla} P=\left(0 I d, \ldots, 0 I d, m_{1} I d, \ldots, m_{n} I d\right)^{t}
$$

que é uma matriz $6 n \times 3$ em cada ponto.

- Para o momento angular total do sistema

$$
\tilde{\nabla} J=\left(-\mathcal{V}_{1}, \ldots,-\mathcal{V}_{n}, \mathcal{Q}_{1}, \ldots, \mathcal{Q}_{n}\right)^{t}
$$

que é uma matriz $6 n \times 3$ em cada ponto, onde

$$
\mathcal{V}_{i}=m_{i}\left(\begin{array}{ccc}
0 & -\psi_{i} & \xi_{i} \\
\psi_{i} & 0 & -\eta_{i} \\
-\xi_{i} & \eta_{i} & 0
\end{array}\right)
$$

e

$$
\mathcal{Q}_{i}=m_{i}\left(\begin{array}{ccc}
0 & -z_{i} & y_{i} \\
z_{i} & 0 & -x_{i} \\
-y_{i} & x_{i} & 0
\end{array}\right) \text {. }
$$

- Para a energia total do sistema

$$
\tilde{\nabla} E=\left(-\nabla_{q_{1}} V, \ldots,-\nabla_{q_{n}} V, m_{i} v_{i}, \ldots, m_{n} v_{n}\right)^{t}
$$

que é uma matriz $6 n \times 1$ em cada ponto.

Com as expressões $(4.20),(4.21),(4.22)$ e (4.23), teremos $\tilde{\nabla} \mathcal{G}_{0}$ na seguinte forma,

$$
\tilde{\nabla} \mathcal{G}_{0}=(\tilde{\nabla} C \vdots \tilde{\nabla} P \vdots \tilde{\nabla} J \vdots \tilde{\nabla} E)
$$


que é uma matriz $6 n \times 10$ em cada ponto.

É simples ver que se os corpos são não colineares as nove primeiras colunas da matriz são linearmente independentes. No caso em que as massas são colineares e as velocidades estão na mesma direção da reta contendo os corpos e temos posto menor que nove. Portanto, quando as massas forem colineares basta escolher $J \neq 0$ e teremos novamente posto máximo nestas nove primeiras colunas.

Assim, assumindo $J \neq 0$ única possibilidade da matriz $\mathcal{G}_{0}$ não ter posto máximo é quando a última coluna é combinação linear as nove primeiras. Isto é, existem três vetores de $\mathbb{R}^{3}, s_{1}, s_{2}$ e $s_{3}$, tais que podemos escrever,

$$
\tilde{\nabla} E=(\tilde{\nabla} C) s_{1}+(\tilde{\nabla} P) s_{2}+(\tilde{\nabla} J) s_{3}
$$

que ainda podemos entender, com relação a cada massa, as seguintes relações,

$$
-\nabla_{q_{i}} V(q)=\frac{m_{i}}{\mu} s_{1}+m_{i} s_{3} \wedge v_{i}, \quad(i=1, \ldots, n),
$$

e

$$
m_{i} v_{i}=m_{i} s_{2}+m_{i} q_{i} \wedge s_{3}, \quad(i=1, \ldots, n) .
$$

Somando no índice $i$ a expressão (4.24) e usando as quantidades conservadas, teremos

$$
0=s_{1}+s_{3} \wedge P=s_{1},
$$

$\operatorname{logo}, s_{1}=(0,0,0)$.

Somando no índice $i$ a expressão (4.25) e usando as quantidades conservadas, teremos

$$
P=0=\mu s_{2}+C \wedge s_{3}=\mu s_{2},
$$

$\operatorname{logo}, s_{2}=(0,0,0)$. 
Usando estes resultados na expressão (4.25), obtemos

$$
m_{i} v_{i}=m_{i} q_{i} \wedge s_{3}, \quad(i=1, \ldots, n),
$$

o que implica na equação (4.24) a seguinte relação,

$$
-\nabla_{q_{i}} V(q)=m_{i}\left(q_{i} \wedge s_{3}\right) \wedge s_{3}, \quad(i=1, \ldots, n) .
$$

Se $s_{3}$ for um vetor unitário poderíamos entender $\left(q_{i} \wedge s_{3}\right) \wedge s_{3}$ como a projeção de $q_{i}$ num plano perpendicular a $s_{3}$. Neste contexto, escrevemos a última relação como,

$$
-\nabla_{q_{i}} V(q)=m_{i}\left|s_{3}\right| \pi_{3} q_{i} \quad(i=1, \ldots, n),
$$

onde, $\pi_{3} q_{i}$ representa a projeção de $q_{i}$ sobre um plano, $\Pi_{3}$, ortogonal a $s_{3}$.

Decorre da equação (4.26) que $\nabla_{q_{i}} V(q) \in \Pi_{3}$, para todo $i=1, \ldots, n$. Isto implica que todos os corpos estão contidos em algum plano paralelo a $\Pi_{3}$. Decorre que as configurações espaciais sempre levarão a valores regulares de $\mathcal{G}_{0}$.

Notemos que, fazendo $\lambda=\left|s_{3}\right|$ na equação (4.26) teremos a seguinte expressão

$$
-\nabla_{q_{i}} V(q)=\lambda m_{i} q_{i}
$$

que escrita na notação para configurações nos dá,

$$
\lambda M Q=-\nabla V(Q)
$$

Uma configuração deste tipo é chamada de configuração crítica.

Novamente, chamamos a atenção, para a semelhança entre as equações (4.4), (4.12), (4.18) e agora (4.27). A menos do fato que na primeira a configuração poderia ser espacial, temos a mesma equação, nos quatro casos. No próximo Capítulo faremos um estudo da equação (4.4), que é a mais geral aqui, pois não necessita ser planar. Esperamos ter motivado a razão pela qual esta equação 
é estudada. 


\section{Capítulo 5}

\section{Configurações Centrais}

Depois que Newton colocou o problema de $n$ corpos, houve muitas tentativas de resolvê-lo. Porém, como foi mostrado no Capítulo 3 é um problema difícil e que não pode ser integrado por quadraturas. No último Capítulo, mostramos algumas soluções particulares, sendo elas a homotética, a de equilíbrio relativo e a homográfica, em todos estes casos a configuração inicial devia respeitar uma mesma equação, a menos da dimensão. Vimos também que os ponto críticos no espaço de fase são dados justamente pela mesma equação que define as tais configurações centrais no plano. As soluções de Euler e Lagrange, comentadas no Capítulo anterior, de certa forma, motivaram a definição de configurações centrais do problema de $n$ corpos, que seriam configurações especiais nas quais conseguimos separar o problema em $n$ problemas de um corpo sob ação de um campo central. No presente Capítulo estudaremos diversos casos de configurações centrais.

\subsection{Definições e Resultados da Literatura}

Definição 5.1.1 Em um instante fixo $t_{0}$, dizemos que $n$ corpos de massas $m_{1}, \ldots, m_{n}$ localizados no referencial baricêntrico, pelos vetores $q_{1}, \ldots, q_{n} /\left(q_{1}, \ldots, q_{n}\right) \in X=\left\{\mathbb{R}^{3 n}-\triangle\right\}$, respectivamente, formam uma configuração central se vale

$$
\ddot{q}_{i}=\lambda q_{i}, \quad \forall i=1, \ldots, n
$$

onde $\lambda=\lambda\left(q_{1}, \ldots, q_{n}\right)$ é uma função não nula. Em outras palavras, podemos dizer que a força resultante sobre o i-ésimo corpo aponta na direção do centro de massa. De modo equivalente, 
podemos escrever a condição de configuração central da seguinte maneira

$$
\lambda q_{i}=-\sum_{k \neq i} \frac{m_{k}\left(q_{i}-q_{j}\right)}{q_{i j}^{3}}, \quad \forall i=1, \ldots, n
$$

Podemos encontrar a forma de $\lambda$ da seguinte maneira, em acordo com as definições e notações do Capítulo 3

$$
m_{i} \ddot{q}_{i}=-\nabla_{i} V
$$

usando a equação (5.1), temos

$$
m_{i} \ddot{q}_{i}=-\nabla_{i} V=\lambda m_{i} q_{i}
$$

Se tomarmos o produto interno por $q_{i}$ em ambos os membros, temos

$$
\left(-\nabla_{i} V\right) \bullet q_{i}=\lambda m_{i} q_{i} \bullet q_{i}
$$

Somando em $i$, lembrando que $V$ é homogênea de grau $(-1)$ e a definição do momento de inércia, temos

$$
V=\lambda(2 I)
$$

logo,

$$
\lambda=\frac{V}{2 I}
$$

Na notação para configurações, a equação (5.1) é exatamente a equação de configurações centrais,

$$
\lambda M q=-\nabla V(q)
$$

Conforme mostramos no Capítulo anterior, Teorema 4.1.4, se temos uma configuração $Q$, solução da equação de configurações centrais, quando multiplicamos esta configuração por uma constante $k \in \mathbb{R}^{+}$, então $k Q$ é solução de uma equação de configurações centrais com constante $\tilde{\lambda}=\lambda / k^{3}$. Assim, tomaremos configurações centrais módulo homotetias centradas no centro de massa em $X$. 
Se fizermos rotações rígidas em torno do centro de massa no espaço de configurações $X$ dos $n$ corpos, claramente iremos preservar a propriedade que define as configurações centrais. Portanto, iremos pensar em configurações centrais módulo rotações rígidas em torno do centro de massa.

Classes de Equivalência: Dizemos que duas configurações centrais são equivalentes se podemos passar de uma à outra fazendo homotetias e/ou rotações rígidas. Assim, daqui por diante, estaremos nos referindo à classe de equivalência de uma configuração central quando encontrarmos um representante da mesma.

Existem vários exemplos conhecidos de configurações centrais. Dentre eles o problema de dois corpos. As configurações colineares de Euler e as equiláteras de Lagrange, que serão mostradas abaixo, formam a cada instante uma configuração central. No decorrer da história do problema de $n$ corpos muitas configurações centrais foram estudadas e catalogadas.

No contexto das soluções homográficas, temos o seguinte resultado que é chamado teorema de Laplace por A. Wintner em [47].

Teorema 5.1.2 Se a solução do problema de n corpos é homográfica, então os corpos formam uma configuração central a cada instante.

A prova deste resultado pode ser vista na seção 4.3, Capítulo anterior. Este teorema torna fundamental o estudo das configurações centrais, pois ele nos diz que se forem conhecidas todas as configurações centrais, então serão conhecidas também todas as possíveis soluções homográficas. Em verdade as soluções homográficas foram durante muito tempo as únicas conhecidas do problema de $n$ corpos. Uma boa referência para o estudo e classificação de soluções homográficas do problema de $n$ corpos é o trabalho de C. Vidal e G. Renildo em [45] no qual também pode ser encontrada uma prova do teorema de Laplace enunciado acima.

Um caso particular do uso de configurações centrais em astrodinâmica é o uso de soluções do tipo equilíbrio relativo como tentativa de modelagem dos anéis de Saturno, como pode ser visto nos trabalhos de D. Saari em [38] e referências do mesmo.

Em seus trabalhos sobre topologia e mecânica S. Smale em [41] provou que as configurações centrais são as imagens inversas dos valores críticos de uma aplicação que associa a cada ponto do 
espaço de fase sua energia e momento angular, uma abordagem mais elaborada que aquela vista na seção 4.4. Assim, o conhecimento das configurações centrais também é importante no entendimento da topologia do problema de $n$ corpos.

Algumas soluções possíveis tais como as de colisão total ou de ejeção nas quais os corpos se afastam mutuamente tem relação direta com configurações centrais. Isto é o que nos mostra os próximos dois resultados de D. Saari em [36] e C. Maccord em [22], respectivamente.

Teorema 5.1.3 Se a solução do problema de n corpos é a de colisão total, então o sistema tende para uma configuração central.

Teorema 5.1.4 Se a solução do problema de $n$ corpos é homotética, então é uma configuração central a cada instante. Em particular, as soluções nas quais os corpos se afastam mutuamente mesmo que não homotéticas tendem para configurações centrais.

Algumas soluções particulares simples envolvem configurações centrais também. Um exemplo está no trabalho de F. Moulton, vide [34], no qual os $n$ corpos estão sobre uma reta. De certo modo, uma generalização da solução retilínea de Euler. O resultado é a contagem das possíveis configurações centrais colineares.

Teorema 5.1.5 Para qualquer escolha de massas positivas no problema de $n$ corpos planar existem exatamente $n ! / 2$ configurações centrais colineares, que correspondem às permutações dos corpos sobre a reta.

Assim, vemos que no caso de configurações centrais colineares, dadas $n$ massas temos um número finito de configurações possíveis. De certa forma isto motiva a seguinte conjectura feita por A. Wintner no caso espacial em [47] e reformulada do S. Smale no caso planar em [39]. Abaixo segue o enunciado de Smale.

Conjectura 5.1.6 Dados $n$ corpos com massas positivas $m_{1}, \ldots, m_{n}$ é finito o número de classes de configurações centrais planares formadas por eles? 
No caso de dois corpos temos apenas uma configuração. Para três corpos temos cinco casos, as três configurações de Euler e as duas de Lagrange. Em 2006 M. Hampton e R. Moeckel em [12] provaram que o número de configurações centrais planares com quatro massas é finito.

Teorema 5.1.7 Existe apenas um número finito de configurações centrais para o problema planar de quatro corpos com massas positivas. Em verdade, o número destas configurações está entre 32 e 8472 .

Recentemente A. Albouy e V. Kaloshin deram uma prova parcial para o caso $n=5$, vide [1].

Teorema 5.1.8 Para a maior parte dos valores de cinco massas existe apenas um número finito de configurações centrais para o problema planar de cinco corpos.

Abaixo colocamos a representação de duas configurações centrais planares para o problema de quatro corpos.
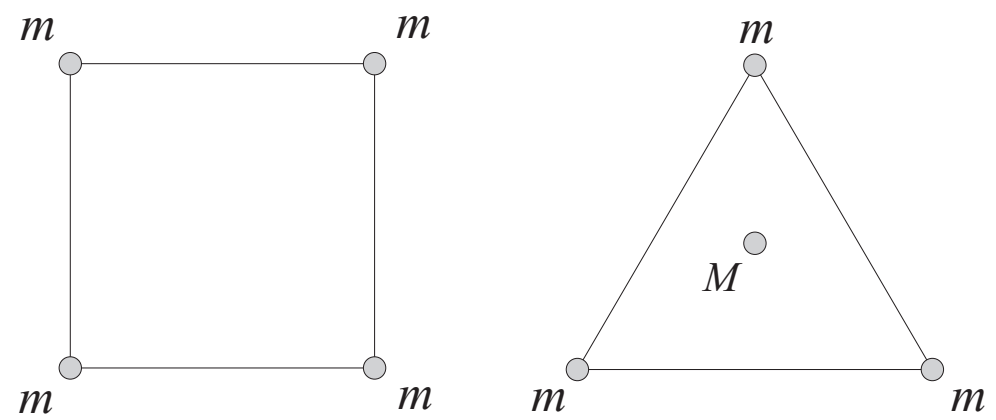

Figura 5.1: Dois exemplos de configurações centrais planares .

Há uma particularidade interessante na configuração de quatro corpos sobre os vértices de um quadrado, que é o seguinte resultado devido a Hampton em [11].

Teorema 5.1.9 A única configuração central cocircular de quatro corpos de massas, com centro de massa no centro da circunferência, é formada por um quadrado com quatro corpos de massas iguais nos seus vértices. 
Existe um resultado devido a G. Roberts em [43] que é quase uma negação à conjectura de Smale, porém ele permite que uma das massas seja igual a -0.25 , o que sai do enunciado da conjectura.

Teorema 5.1.10 No problema planar de 5-corpos se quatro massas são iguais a 1 e a quinta é igual a-0.25, então existe uma familia a um parâmetro de configurações centrais, com as quatro massas iguais nos vértices de um losango e a quinta, negativa, no centro.

Segue a representação do resultado de Roberts, vide figura 5.2.

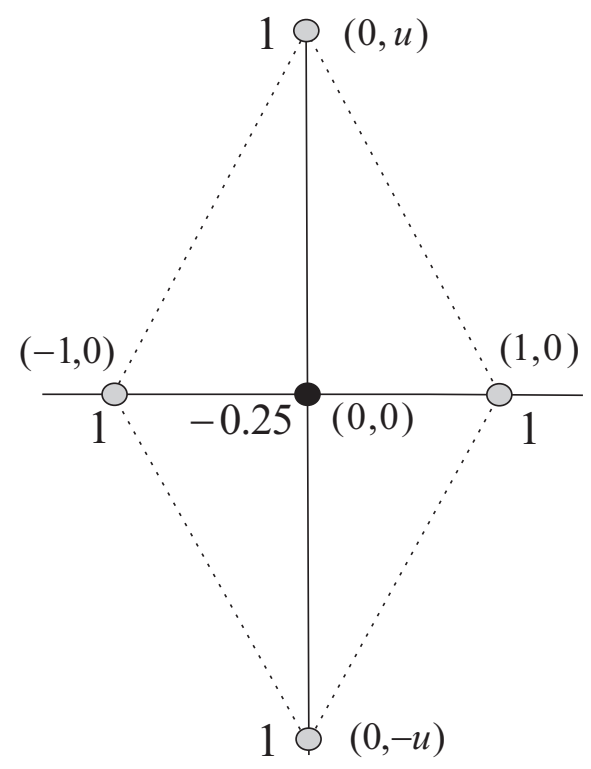

Figura 5.2: Exemplo de Roberts: temos configurações centrais para todos os valores positivos de $u$.

\subsection{Equações De Andoyer e Aplicações}

Primeiramente, vamos definir um conjunto de equações que são muito úteis na determinação de configurações centrais. São as chamadas equações de Andoyer, uma referência para elas está nos trabalhos de Y. Hagihara em [10] ou o trabalho original de Andoyer [3]. Aqui vamos apresentar as equações de Andoyer apenas para áreas e volumes em $\mathbb{R}^{3}$, mas podemos generalizar facilmente estas expressões para hipervolumes de $\mathbb{R}^{d} \operatorname{com} d>3$. 
Definição 5.2.1 As equações de Andoyer com áreas em $\mathbb{R}^{3}$ são dadas por

$$
f_{i j}=\sum_{k \neq i, j} m_{k}\left(R_{i k}-R_{j k}\right) \Delta_{i j k}=0,1 \leq i<j \leq n
$$

onde $R_{i j}=R_{j i}=\left|q_{i}-q_{j}\right|^{-3}$ e $\Delta_{i j k}=\left(q_{i}-q_{j}\right) \wedge\left(q_{i}-q_{k}\right)$ é duas vezes a área com sinal do triângulo formado por $m_{i}, m_{j}$ e $m_{k}$.

Estas equações formam um conjunto de $n(n-1) / 2$ equações. Apesar de aumentarmos o número de equações, em certos casos é mais simples operar com estas.

Definição 5.2.2 As equações de Andoyer com volumes em $\mathbb{R}^{3}$ são dadas por

$$
f_{i j h}=\sum_{k \neq i, j, h} m_{k}\left(R_{i k}-R_{j k}\right) \Delta_{i j h k}=0,1 \leq i<j \leq n, h=1, \ldots, n
$$

onde $R_{i j}=R_{j i}=\left|q_{i}-q_{j}\right|^{-3}$ e $\Delta_{i j h k}=\left(q_{i}-q_{j}\right) \wedge\left(q_{j}-q_{h}\right) \bullet\left(q_{h}-q_{k}\right)$ é seis vezes o volume com sinal do tetraedro formado por $m_{i}, m_{j}, m_{h}$ e $m_{k}$.

Estas equações formam um conjunto de $n(n-1)(n-2) / 2$ equações. Apesar de aumentarmos o número de equações, em certos casos é mais simples operar com estas.

Observação 5.2.3 Aqui chamamos estes dois conjuntos de equações algébricas de equação de Andoyer. Existem divergências na literatura sobre a autoria destas equações, as quais são também conhecidas como equações de Dziobek, Laura-Andoyer ou de Routh. Optamos por manter apenas o nome Andoyer.

Vamos mostrar agora que as expressões em (5.2) é equivalente a expressão (5.3).

Teorema 5.2.4 Considerando um sistema de $n$ massas não colineares, então elas formam uma configuração central se, e somente se, respeitam a seguinte condição

$$
f_{i j}=0, \quad \forall i, j(1 \leq i<j \leq n)
$$


Prova: Suponhamos que as $n$ massas formam uma configuração central planar. Portanto, existe $\lambda$ tal que

$$
\lambda q_{i}=-\sum_{k \neq i} m_{k} R_{i k}\left(q_{i}-q_{k}\right)
$$

Naturalmente, podemos retirar um termo da soma acima, para $j \neq i$, obtendo

$$
\lambda q_{i}=-\sum_{k \neq i, j} m_{k} R_{i k}\left(q_{i}-q_{k}\right)-m_{j} R_{i j}\left(q_{i}-q_{j}\right)
$$

Do mesmo modo para $q_{j}$ com $j \neq i$, obtemos

$$
\lambda q_{j}=-\sum_{k \neq j, i} m_{k} R_{j k}\left(q_{j}-q_{k}\right)-m_{i} R_{j i}\left(q_{j}-q_{i}\right)
$$

Considere agora a subtração de (5.5) por (5.6), donde

$$
\lambda\left(q_{i}-q_{j}\right)=-\sum_{k \neq i, j} m_{k}\left[R_{i k}\left(q_{i}-q_{k}\right)-R_{j k}\left(q_{j}-q_{k}\right)\right]-\left[m_{j} R_{i j}-m_{i} R_{j i}\right]\left(q_{i}-q_{j}\right) .
$$

Tomando produto o vetorial por $\left(q_{i}-q_{j}\right)$ em ambos os membros de (5.7), temos

$$
0=-\sum_{k \neq i, j} m_{k}\left(R_{i k}-R_{j k}\right) \Delta_{i j k}=-f_{i j}
$$

Logo, $f_{i j}=0$, para todo $1 \leq i<j \leq n$.

Reciprocamente, consideremos que as equações de Andoyer são verificadas

$$
f_{i j}=\sum_{k \neq i, j}^{n} m_{k}\left(R_{i k}-R_{j k}\right)\left(q_{i}-q_{j}\right) \wedge\left(q_{i}-q_{k}\right)=0
$$

para $1 \leq i<j \leq n$, as quais podem ser escritas da forma

$$
\sum_{k \neq i, j}^{n} m_{k} R_{i k}\left(q_{i}-q_{j}\right) \wedge\left(q_{i}-q_{k}\right)=\sum_{k \neq i, j}^{n} m_{k} R_{j k}\left(q_{i}-q_{j}\right) \wedge\left(q_{i}-q_{k}\right) .
$$


No membro esquerdo da equação (5.8) podemos incluir o termo em $j$ sem alterar a igualdade. Da mesma forma, podemos inserir o termo em $i$ no membro direito. Assim, temos

$$
\sum_{k \neq i}^{n} m_{k} R_{i k}\left(q_{i}-q_{j}\right) \wedge\left(q_{i}-q_{k}\right)=\sum_{k \neq j}^{n} m_{k} R_{j k}\left(q_{i}-q_{j}\right) \wedge\left(q_{i}-q_{k}\right),
$$

ou seja,

$$
\left(q_{i}-q_{j}\right) \wedge \sum_{k \neq i}^{n} m_{k} R_{i k}\left(q_{i}-q_{k}\right)=\sum_{k \neq j}^{n} m_{k} R_{j k}\left[q_{i} \wedge\left(q_{j}-q_{k}\right)+\left(q_{j} \wedge q_{k}\right)\right] .
$$

Denotando $\nabla_{i} V$ por $F_{i}$, vemos que a igualdade (5.9) pode ser escrita como

$$
\left(q_{i}-q_{j}\right) \wedge \frac{F_{i}}{m_{i}}=\sum_{k \neq j}^{n} m_{k} R_{j k}\left[q_{i} \wedge\left(q_{j}-q_{k}\right)+\left(q_{j} \wedge q_{k}\right)\right] .
$$

Podemos inserir à direita da igualdade (5.9) o termo $-q_{j}$ sem alterá-la, obtendo

$$
\left(q_{i}-q_{j}\right) \wedge \frac{F_{i}}{m_{i}}=\sum_{k \neq j}^{n} m_{k} R_{j k}\left[q_{i} \wedge\left(q_{j}-q_{k}\right)+q_{j} \wedge\left(-q_{j}+q_{k}\right)\right] .
$$

rearranjando o lado direito temos

$$
\begin{aligned}
\left(q_{i}-q_{j}\right) \wedge \frac{F_{i}}{m_{i}} & =\sum_{k \neq j}^{n} m_{k} R_{j k}\left[q_{i} \wedge\left(q_{j}-q_{k}\right)-q_{j} \wedge\left(q_{j}-q_{k}\right)\right] \\
& =\sum_{k \neq j}^{n} m_{k} R_{j k}\left[\left(q_{i}-q_{j}\right) \wedge\left(q_{j}-q_{k}\right)\right] \\
& =\left(q_{i}-q_{j}\right) \wedge \frac{F_{j}}{m_{j}}
\end{aligned}
$$

donde

$$
\left(q_{i}-q_{j}\right) \wedge \frac{F_{i}}{m_{i}}=\left(q_{i}-q_{j}\right) \wedge \frac{F_{j}}{m_{j}}
$$

a qual implica que

$$
\left(q_{i}-q_{j}\right) \wedge\left(m_{j} F_{i}-m_{i} F_{j}\right)=0 .
$$


Efetuando o produto vetorial termo a termo em (5.10), obtemos

$$
q_{i} \wedge m_{j} F_{i}-q_{i} \wedge m_{i} F_{j}-q_{j} \wedge m_{j} F_{i}+q_{j} \wedge m_{i} F_{j}=0
$$

de onde temos

$$
m_{j} q_{i} \wedge F_{i}-m_{i} q_{i} \wedge F_{j}-m_{j} q_{j} \wedge F_{i}+m_{i} q_{j} \wedge F_{j}=0
$$

Somando em $j$ com $j \neq i$, temos

$$
\left(M-m_{i}\right) q_{i} \wedge F_{i}-m_{i} q_{i} \wedge \sum_{j \neq i}^{n} F_{j}-\left(\sum_{j \neq i}^{n} m_{j} q_{j}\right) \wedge F_{i}+m_{i} \sum_{j \neq i}^{n} q_{j} \wedge F_{j}=0
$$

onde $M$ é a massa total. Como o centro de massa está na origem do referencial, temos

$$
\sum_{j=1}^{n} m_{j} q_{j}=0 \Longrightarrow \sum_{j \neq i}^{n} m_{j} q_{j}=-m_{i} q_{i}
$$

Visto que o espaço é homogêneo e isotrópico e o sistema é isolado, temos que as quantidades de momento linear total e momento angular total são conservadas. Então, respectivamente, temos

$$
\sum_{j=1}^{n} F_{j}=0 \Longrightarrow \sum_{j \neq i}^{n} F_{j}=-F_{i}
$$

$\mathrm{e}$

$$
\sum_{j=1}^{n}\left(q_{j} \wedge F_{j}\right)=0 \Longrightarrow \sum_{j \neq i}^{n}\left(q_{j} \wedge F_{j}\right)=\left(-q_{i} \wedge F_{i}\right) .
$$

Substituindo (5.12), (5.13) e (5.14) em (5.11), obtemos

$$
M q_{i} \wedge F_{i}-m_{i} q_{i} \wedge F_{i}+m_{i} q_{i} \wedge F_{i}+m_{i} q_{i} \wedge F_{i}-m_{i} q_{i} \wedge F_{i}=0
$$

Desta forma, $M q_{i} \wedge F_{i}=0$, $\log q_{i}$ e $F_{i}$ são paralelos, ou seja, $F_{i}=\lambda_{i} q_{i}$, ou ainda $\ddot{q}_{i}=\left(\lambda_{i} / m_{i}\right) q_{i}$. De (5.10), decorre

$$
\left(\frac{\lambda_{i}}{m_{i}} q_{i}-\frac{\lambda_{j}}{m_{j}} q_{j}\right) \wedge\left(q_{i}-q_{j}\right)=0
$$


Assim,

$$
-\frac{\lambda_{i}}{m_{i}} q_{i} \wedge q_{j}-\frac{\lambda_{j}}{m_{j}} q_{j} \wedge q_{i}=0
$$

o que implica em

$$
\left(\frac{\lambda_{i}}{m_{i}}-\frac{\lambda_{j}}{m_{j}}\right)\left(q_{j} \wedge q_{i}\right)=0
$$

Se $q_{j}$ é paralelo a $q_{i}$, a igualdade acima se verifica facilmente, porém como por hipótese não temos uma configuração colinear, existem pelo menos três massas não colineares. Se $q_{i}$ e $q_{j}$ são não colineares, temos que

$$
\frac{\lambda_{i}}{m_{i}}=\frac{\lambda_{j}}{m_{j}}=\lambda
$$

para todo $i, j$. Portanto,

$$
\ddot{q}_{i}=\lambda q_{i}
$$

para todo $i=1,2, \ldots, n$, como queríamos provar.

Uma prova análoga pode ser feita para o seguinte teorema.

Teorema 5.2.5 Considerando um sistema de $n$ massas não coplanares, então elas formam uma configuração central se, e somente se, respeitam a seguinte condição

$$
f_{i j h}=0, \quad \forall i, j, h(1 \leq i<j \leq n) e(h=1, \ldots, n) .
$$

As equações de Andoyer são muito úteis quando queremos calcular configurações onde sabemos que existem simetrias a priori nas posições. Como primeira aplicação destas equações, vamos calcular as configurações centrais não colineares do problema de três corpos.

Teorema 5.2.6 A única configuração central de três massas, não colinear, é o triângulo equilátero com massas arbitrárias nos vértices. 
Prova: Para este caso teremos 3 equações de Andoyer com áreas, as quais seguem,

$$
\begin{aligned}
& f_{12}=m_{3}\left(R_{13}-R_{23}\right) \Delta_{123}=0 \\
& f_{13}=m_{2}\left(R_{12}-R_{32}\right) \Delta_{132}=0
\end{aligned}
$$

e

$$
f_{23}=m_{1}\left(R_{21}-R_{31}\right) \Delta_{321}=0
$$

Como, por hipótese, a configuração é não colinear os termos $\Delta_{i j k}$ são todos não nulos. Além disto consideramos apenas os casos onde as massas são positivas, logo

$$
\begin{aligned}
& f_{12}=0 \Leftrightarrow R_{13}=R_{23} \Leftrightarrow q_{13}=q_{23}, \\
& f_{13}=0 \Leftrightarrow R_{12}=R_{32} \Leftrightarrow q_{12}=q_{32} .
\end{aligned}
$$

Assim, $q_{12}=q_{13}=q_{23}$, o que implica que os corpos estão nos vértices de um triângulo equilátero. E mais que isto, a configuração não depende do valor das massas.

Como citado acima estas são as chamadas configurações equiláteras de Lagrange, segue na figura 5.3 a representação das duas possíveis configurações equiláteras, uma vez que não incluímos reflexões nas classes de equivalência, existem duas.

Observando a simplicidade envolvida na prova deste teorema podemos nos perguntar se existe um análogo com quatro corpos no espaço? A resposta é sim e pode ser enunciada no seguinte teorema.

Teorema 5.2.7 A única configuração central espacial de quatro massas, não coplanar, é o tetraedro regular com massas arbitrárias nos vértices. 


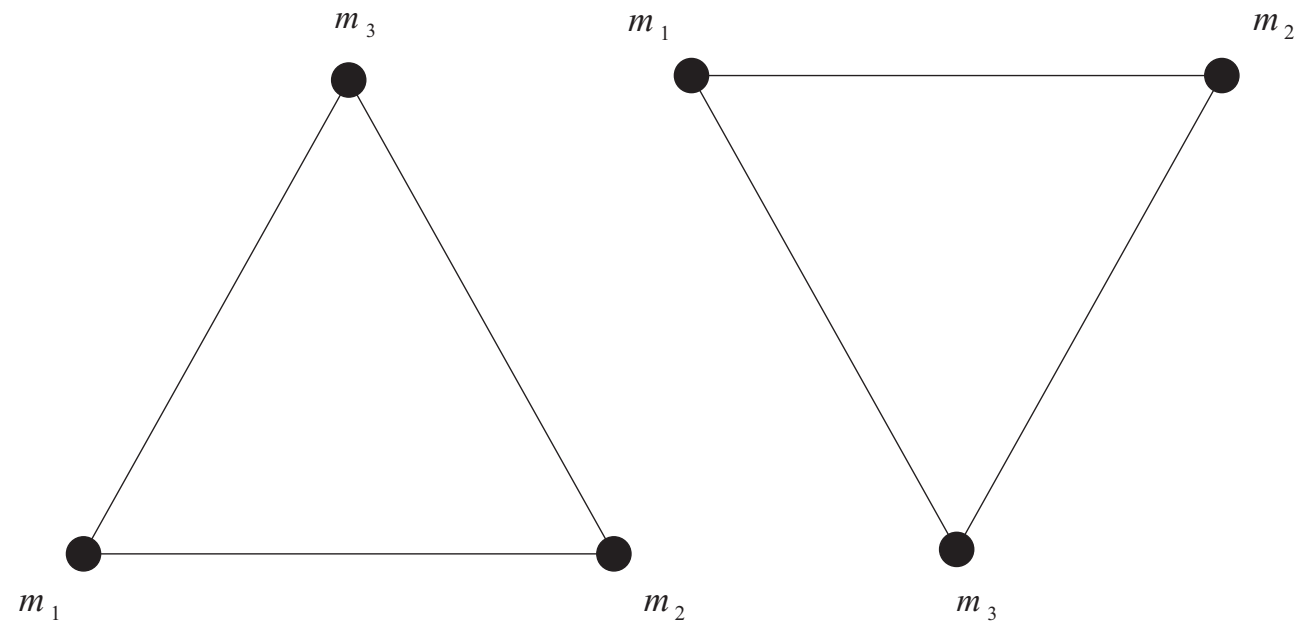

Figura 5.3: As duas configurações equiláteras.

A prova é análoga ao caso anterior, bastando listar as equações de Andoyer com volumes. Este resultado é devido a Lemam-Filhès e pode ser encontrado em [10]. Segue abaixo uma figura com a representação de uma configurações centrais para este caso.

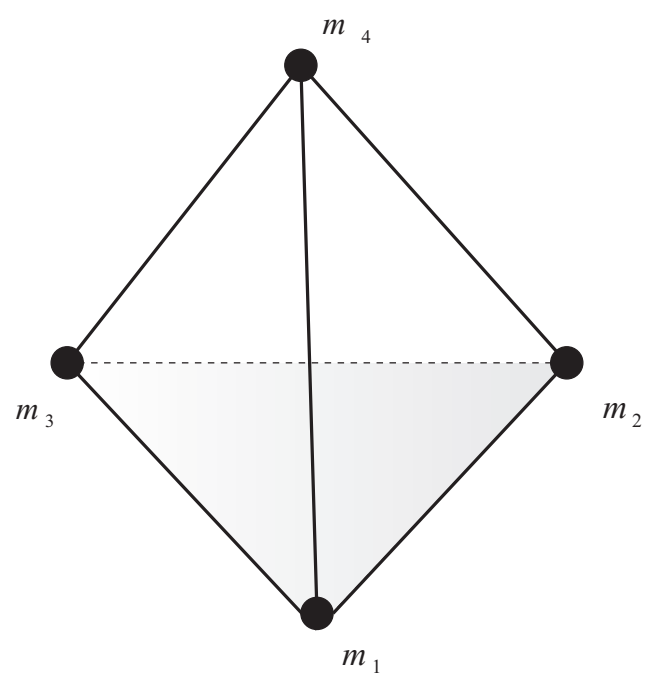

Figura 5.4: Configuração central tetraédrica.

Vamos enunciar e provar, usando as equações de Andoyer, um resultado devido a Conley, segundo Moeckel em [32]. Conhecido como o teorema do perpendicular bissetor. Como veremos, este teorema é muito útil para provar a não existência de certas configurações centrais. 
Teorema 5.2.8 Considere uma configuração central planar, formadas por $n$ massas positivas $m_{1}, \ldots, m_{n}$. Escolha duas massas $m_{i}$ e $m_{j}$ com posições $q_{i}$ e $q_{j}$, respectivamente. Trace a reta que contém estas duas massas e a reta que bissecta o segmento $\overline{q_{i} q_{j}}$, de acordo com as figuras 5.5 e 5.6. Estas duas retas definem dois cones no plano. Então, se existem massas num dos cones abertos, devemos ter também massas no outro cone. Em outras palavras, se as $(n-2)$ massas restantes pertencessem a apenas um cone aberto não teríamos uma configuração central.

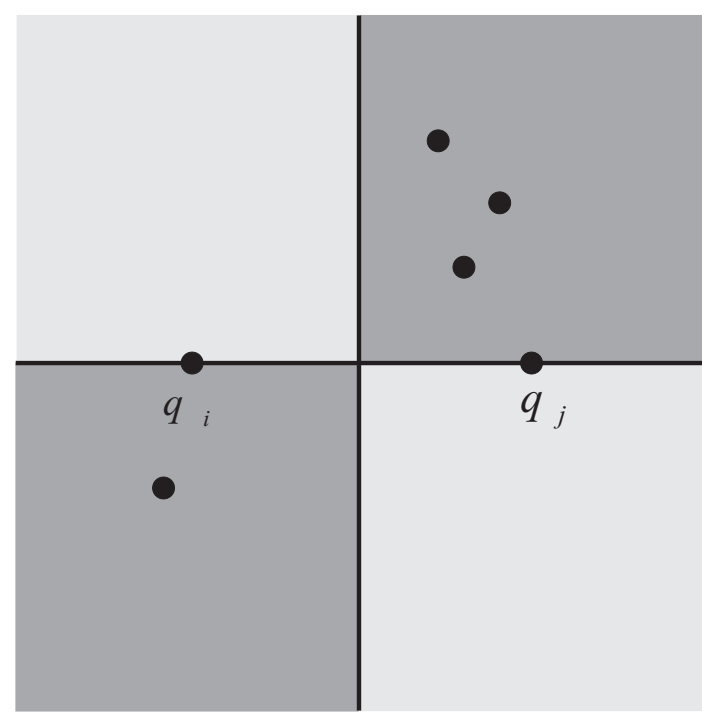

Figura 5.5: Não é configuração central.

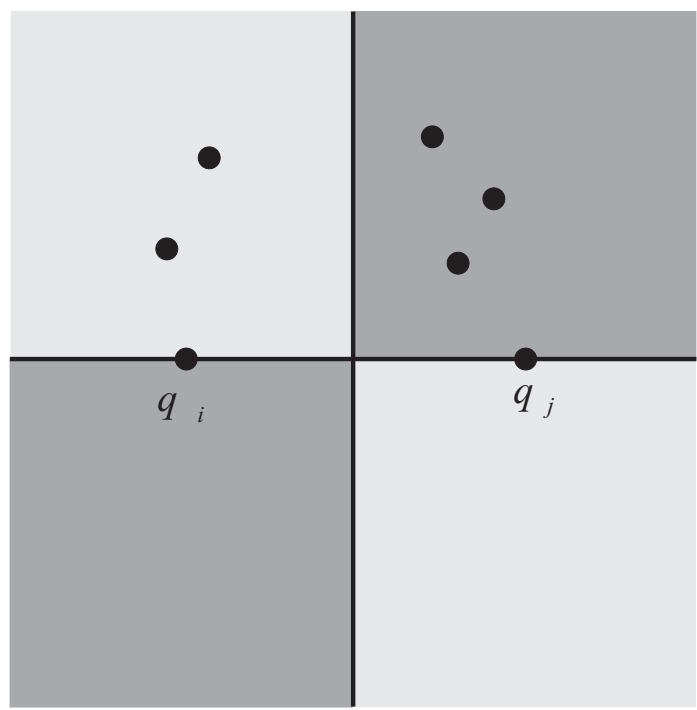

Figura 5.6: Poderia ser configuração central.

Prova: Não há perda de generalidade se renomearmos os índices $i$ e $j$ por 1 e 2 . Suponha, por contradição, que as massas $m_{3}, \ldots, m_{n}$ estão ou num único cone aberto ou sobre o eixo bissetor, mas não todas sobre o eixo.

Como por hipótese temos uma configuração central planar, todas as $n(n-1) / 2$ equações de Andoyer são satisfeitas, em particular

$$
f_{12}=\sum_{k=3}^{n} m_{k}\left(R_{1 k}-R_{2 k}\right) \Delta_{12 k}=0 .
$$

Podemos olhar as retas passando pelos corpos e bissectando o segmento como eixos que dividem o plano em 4 quadrantes ordenados ciclicamente, como usual. Assim um cone aberto é formado pela união do primeiro e terceiro quadrantes abertos, e o outro pela união do segundo e quarto 
quadrantes abertos. Assim, vamos supor que existem massas no primeiro e terceiro quadrantes e algumas sobre os eixos, possivelmente nenhuma.

Vamos tomar a soma acima em três parcelas, da seguinte forma, o índice $l$ denotará termos no primeiro quadrante, $k$ no terceiro e $i$ os termos sobre a bissetriz. Desta forma,

$$
f_{12}=\sum_{l} m_{l}\left(R_{1 l}-R_{2 l}\right) \Delta_{12 l}+\sum_{k} m_{k}\left(R_{1 k}-R_{2 k}\right) \Delta_{12 k}+\sum_{i} m_{i}\left(R_{1 i}-R_{2 i}\right) \Delta_{12 i}=0 .
$$

Primeiramente, note que os termos da terceira soma são todos nulos, pois sobre a bissetriz $R_{1 i}=R_{2 i}$. Donde, teremos

$$
f_{12}=\sum_{l} m_{l}\left(R_{1 l}-R_{2 l}\right) \Delta_{12 l}+\sum_{k} m_{k}\left(R_{1 k}-R_{2 k}\right) \Delta_{12 k}=0
$$

Estudemos o sinal do termos na primeira soma, $\Delta_{12 l}>0$, para todo $l$ e

$$
q_{1 l}>q_{2 l} \Rightarrow R_{1 l}<R_{2 l}
$$

logo, todos os coeficientes das massas, na primeira soma, tem sinal positivo.

Para os termos da segunda soma, temos $\Delta_{12 k}<0$, para todo $k$ e

$$
q_{1 k}<q_{2 l} \Rightarrow R_{1 l}>R_{2 l}
$$

logo, todos os coeficientes das massas, na segunda soma, tem sinal positivo, também. Mas, isto é uma contradição com a positividade das massas.

Este teorema é muito útil quando desejamos decidir se certos casos de configurações podem ou não ser configurações centrais. A própria configuração equilátera de Lagrange sai trivialmente como corolário deste teorema. Veremos alguns casos do uso deste resultado no decorrer do Capítulo. 


\subsection{Configurações Centrais do Tipo Pipa}

Vamos seguir os trabalhos J. Bernat, J. Llibre e E. Pérez-Chavela em [4] no qual eles definem o que vem a ser configurações planares do tipo pipa no problema de quatro corpos. Os resultados aqui expostos podem ser encontrados em [26]. Para seguir a notação destes artigos usaremos daqui por diante $r_{i}$ para denotar a posição das massas.

Estudaremos configurações centrais planares não colineares do problema de 4-corpos que têm a forma de pipa, ou simplesmente configurações centrais do tipo pipa, as quais podem ser definidas como àquelas que têm um eixo de simetria passando por duas das massas. A configuração do tipo pipa é chamada convexa se nenhum dos corpos está localizado no interior do fecho convexo dos outros três. Veja figura 5.7. Caso contrário, dizemos que a configuração do tipo pipa é côncava. Veja figura 5.8.

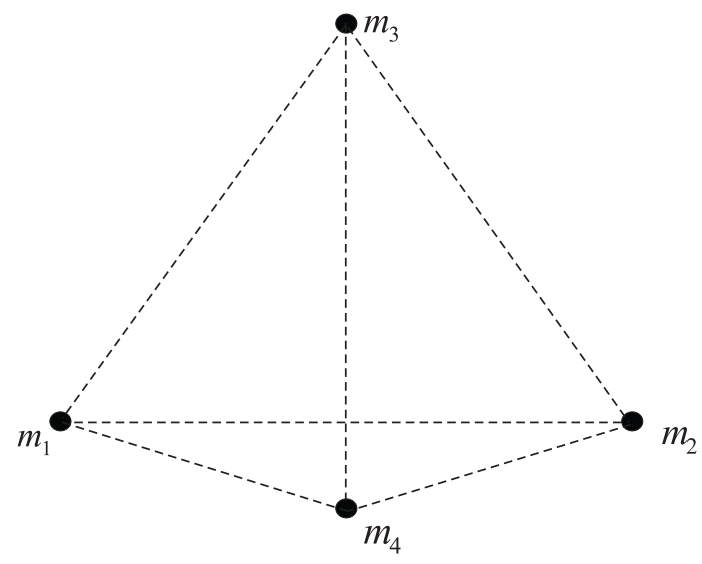

Figura 5.7: Pipa convexa.

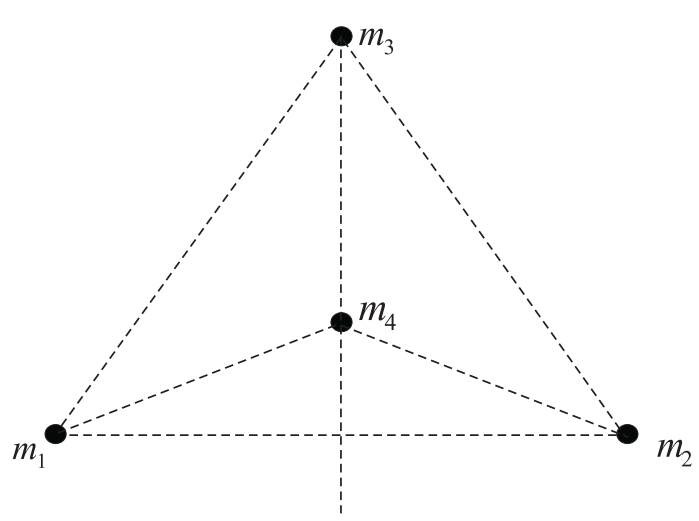

Figura 5.8: Pipa côncava.

Suporemos que as massas $m_{3}$ e $m_{4}$ estarão sobre a reta de simetria das configurações do tipo pipa, sendo que a posição de $m_{3}$ estará fixa, conforme a figura 5.9. O resultado é o seguinte.

Teorema 5.3.1 Considere quatro massas $m_{1}, m_{2}, m_{3}$ e $m_{4}$ localizadas em $(-x, 0),(x, 0),(0, \sqrt{3} / 2)$ $e(0, y), \operatorname{com} x>0$ e $y<\sqrt{3} / 2$, de acordo com a figura 5.9. Valem as seguintes afirmações:

1. Para cada

$$
x_{0} \in\left(\frac{2 \sqrt{3}-3}{2}, \frac{1}{2}\right) \cup\left(\frac{1}{2}, \frac{2 \sqrt{3}+3}{2}\right) \text {, }
$$


existe um intervalo aberto não vazio $I_{x_{0}}$ tal que, para cada $y_{0} \in I_{x_{0}}$, existem massas positivas $m_{1}=m_{2}, m_{3}$ e $m_{4}$ de modo que os quatro corpos, como na figura 5.9, estão numa configuração central do tipo pipa;

2. Para $x_{0}=1 / 2$ e $y_{0}=\sqrt{3} / 6$, existem massas positivas $m_{1}=m_{2}=m_{3}$ e $m_{4}$ de modo que os quatro corpos, como na figura 5.9, estão numa configuração central do tipo pipa côncava;

3. Para $x_{0}=\sqrt{3} / 2$ e $y_{0}=-\sqrt{3} / 2$, existem massas positivas $m_{1}=m_{2}=m_{3}=m_{4}$ de modo que os quatro corpos, como na figura 5.9, estão numa configuração central do tipo pipa convexa.

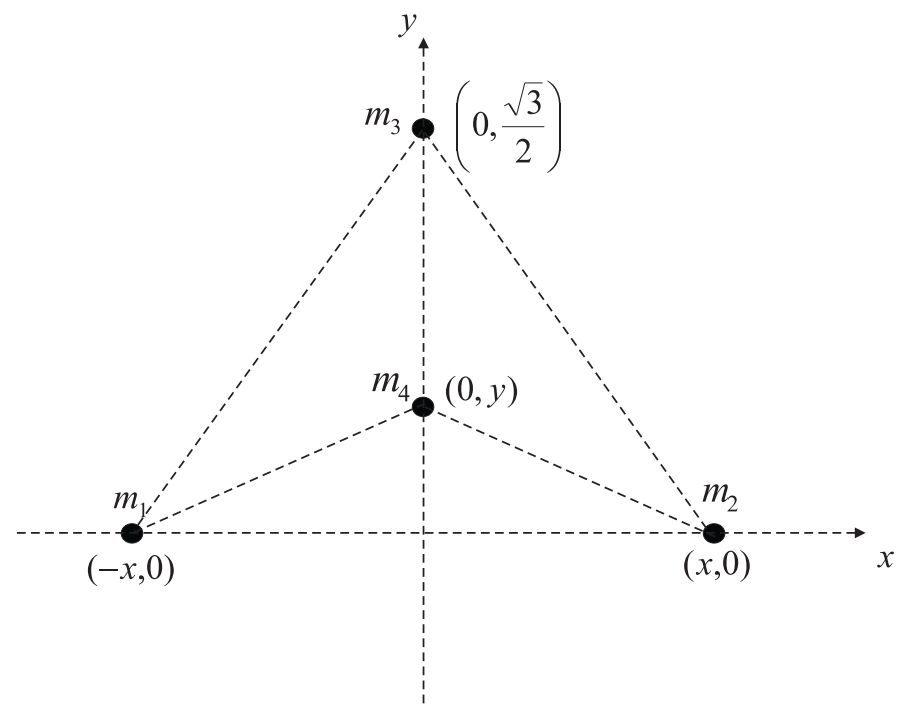

Figura 5.9: Configuração central do tipo pipa.

Prova: Nesta seção faremos a prova do Teorema 5.3.1 utilizando as equações de Andoyer (5.3). Para o problema planar de 4-corpos (5.3) é um conjunto de 6 equações. As configurações do tipo pipa, como na figura 5.9, sem colisões, devem satisfazer, entre outras, as seguintes relações

$$
r_{13}=r_{23}, \quad r_{14}=r_{24}, \quad \Delta_{143}=\Delta_{234} .
$$

Consideremos as equações de Andoyer de nosso problema

$$
f_{12}=m_{3}\left(R_{13}-R_{23}\right) \Delta_{123}+m_{4}\left(R_{14}-R_{24}\right) \Delta_{124}=0,
$$




$$
\begin{aligned}
& f_{13}=m_{2}\left(R_{12}-R_{32}\right) \Delta_{132}+m_{4}\left(R_{14}-R_{34}\right) \Delta_{134}=0, \\
& f_{14}=m_{2}\left(R_{12}-R_{42}\right) \Delta_{142}+m_{3}\left(R_{13}-R_{43}\right) \Delta_{143}=0, \\
& f_{23}=m_{1}\left(R_{21}-R_{31}\right) \Delta_{231}+m_{4}\left(R_{24}-R_{34}\right) \Delta_{234}=0, \\
& f_{24}=m_{1}\left(R_{21}-R_{41}\right) \Delta_{241}+m_{3}\left(R_{23}-R_{43}\right) \Delta_{243}=0, \\
& f_{34}=m_{1}\left(R_{31}-R_{41}\right) \Delta_{341}+m_{2}\left(R_{32}-R_{42}\right) \Delta_{342}=0 .
\end{aligned}
$$

Usando as relações acima, podemos reescrever a equação (5.20) da seguinte maneira

$$
\left(m_{1}-m_{2}\right)\left(R_{31}-R_{41}\right) \Delta_{341}=0 .
$$

Nas hipóteses do Teorema 5.3.1, o termo $\Delta_{134}$ nunca se anula, daí $m_{1}=m_{2}$ ou $R_{13}=R_{14}$. Se $R_{13}=R_{14}$, temos um quadrado, que é uma configuração central se, e somente se, $m_{1}=m_{2}=m_{3}=$ $m_{4}$ (veja item 3 do Teorema 5.3.1). Assim, podemos supor, sem perda de generalidade, que

$$
m_{1}=m_{2}=1
$$

A equação (5.15) é trivialmente satisfeita. Usando as relações de simetria acima, temos

$$
f_{13}=0 \Leftrightarrow f_{23}=0
$$

e

$$
f_{14}=0 \Leftrightarrow f_{24}=0 .
$$

Portanto, é suficiente encontrar soluções para (5.16) e (5.17), com valores positivos para as massas $m_{3}$ e $m_{4}$. Vamos reescrever estas equações de modo a obter $m_{3}$ e $m_{4}$ como funções das posições

$$
m_{3}=\frac{\left(R_{12}-R_{42}\right) \Delta_{142}}{\left(R_{43}-R_{13}\right) \Delta_{143}}
$$




$$
m_{4}=\frac{\left(R_{12}-R_{32}\right) \Delta_{132}}{\left(R_{34}-R_{14}\right) \Delta_{134}}
$$

Desejamos encontrar, se existirem, as regiões no semiplano $x>0$ para as quais teremos valores positivos para ambas as massas. Devemos, então, estudar o sinal dos termos envolvidos nas expressões de $m_{3}$ e $m_{4}$ em (5.21) e (5.22), respectivamente.

Substituindo as coordenadas, conforme indicações da figura 5.9, temos

$$
\begin{gathered}
\left(R_{12}-R_{42}\right)<0 \Leftrightarrow(x, y) \in\{x>0,-\sqrt{3} x<y<\sqrt{3} x\}, \\
\left(R_{34}-R_{14}\right)<0 \Leftrightarrow(x, y) \in\left\{x>0, y<-\frac{\sqrt{3}}{3} x^{2}+\frac{\sqrt{3}}{4}\right\}, \\
\left(R_{43}-R_{13}\right)<0 \Leftrightarrow(x, y) \in\left\{x>0, y<\frac{\sqrt{3}-\sqrt{3+4 x^{2}}}{2}\right\}, \\
\left(R_{12}-R_{32}\right)<0 \Leftrightarrow(x, y) \in\left\{x>\frac{1}{2}, y<\frac{\sqrt{3}}{2}\right\} .
\end{gathered}
$$

Para o sinal das áreas orientadas consideremos primeiramente $0<y<\sqrt{3} / 2$. Neste caso, nas expressões (5.21) e (5.22) vale

$$
\Delta_{142}<0, \quad \Delta_{143}>0, \quad \Delta_{132}<0, \quad \Delta_{134}<0
$$

Assim, comparando o sinal de cada um dos termos das equações (5.21) e (5.22), teremos que se $(x, y) \in A_{1} \cup A_{2}$, as massas $m_{3}$ e $m_{4}$ serão positivas, onde

$$
\begin{gathered}
A_{1}=\left\{(x, y) \in \mathbb{R}^{2}: \frac{2 \sqrt{3}-3}{2}<x<\frac{1}{2},-\frac{\sqrt{3}}{3} x^{2}+\frac{\sqrt{3}}{4}<y<\sqrt{3} x\right\}, \\
A_{2}=\left\{(x, y) \in \mathbb{R}^{2}: \frac{1}{2}<x<\frac{\sqrt{3}}{2}, 0<y<-\frac{\sqrt{3}}{3} x^{2}+\frac{\sqrt{3}}{4}\right\} .
\end{gathered}
$$


Consideremos agora $y<0$. Neste caso, nas expressões (5.21) e (5.22), temos

$$
\Delta_{142}>0, \quad \Delta_{143}>0, \quad \Delta_{132}<0, \quad \Delta_{134}<0
$$

Novamente, comparando o sinal de cada um dos termos das equações (5.21) e (5.22), teremos que se $(x, y) \in A_{3}=B_{1} \cup B_{2}$, as massas $m_{3}$ e $m_{4}$ serão positivas, onde

$$
\begin{gathered}
B_{1}=\left\{(x, y) \in \mathbb{R}^{2}: \frac{1}{2}<x \leq \frac{3}{2},-\sqrt{3} x<y<\frac{\sqrt{3}-\sqrt{3+4 x^{2}}}{2}\right\}, \\
B_{2}=\left\{(x, y) \in \mathbb{R}^{2}: \frac{3}{2} \leq x \leq \frac{2 \sqrt{3}+3}{2},-\sqrt{3} x<y<-\frac{\sqrt{3}}{3} x^{2}+\frac{\sqrt{3}}{4}\right\} .
\end{gathered}
$$

O caso $y=0$ não precisa ser considerado, pois, não ocorre configuração central do tipo pipa, devido ao teorema do perpendicular bissetor, conforme o Teorema 5.2.8.

Finalmente, concluímos que se $\left(x_{0}, y_{0}\right) \in A_{1} \cup A_{2} \cup A_{3}$, vide figura 5.10, então as massas

$$
m_{1}=1, \quad m_{2}=1, \quad m_{3}=m_{3}\left(x_{0}, y_{0}\right), \quad m_{4}=m_{4}\left(x_{0}, y_{0}\right),
$$

com posições

$$
r_{1}=\left(-x_{0}, 0\right), \quad r_{2}=\left(x_{0}, 0\right), \quad r_{3}=\left(0, \frac{\sqrt{3}}{2}\right), \quad r_{4}=\left(0, y_{0}\right),
$$

formam uma configuração central do tipo pipa, como mostrada na figura 5.9. O intervalo $I_{x_{0}}$ do enunciado do teorema é obtido tomando a interseção da reta $x=x_{0}$ com a região aberta $A_{i}$, $i=1,2,3$. Isso demonstra o item 1 do Teorema 5.3.1.

Para a prova do item 2, observemos que, neste caso, valem $r_{12}=r_{13}=r_{23}=1, r_{14}=r_{24}$ e $\Delta_{143}=\Delta_{234}$. Assim, $f_{12}=0$ é trivialmente satisfeita. De $f_{13}=0$ e de $f_{23}=0$ resultam, respectivamente, $R_{14}=R_{34}$ e $R_{24}=R_{34}$. Portanto, $R_{14}=R_{24}=R_{34}$. Assim, de $f_{24}=0$ e $f_{34}=0$, temos $m_{1}=m_{3}$ e $m_{1}=m_{2}$, respectivamente. Logo, $m_{1}=m_{2}=m_{3}$. De posse dessas conclusões sobre as massas e sobre as distâncias, segue que $f_{14}=0$ também é trivialmente satisfeita. Isso conclui a prova do item 2 do Teorema 5.3.1. 
Passemos agora à prova do item 3 do Teorema 5.3.1. Neste caso, os 4 corpos estão sobre os vértices de um quadrado, de modo que as seguintes igualdades são obtidas: $r_{12}=r_{34}, r_{13}=r_{23}=$ $r_{14}=r_{24}$ e $\Delta_{123}=\Delta_{143}=\Delta_{234}=\Delta_{142}$. Substituindo essas informações nas equações de Andoyer resulta que $f_{12}=0$ e $f_{34}=0$ são trivialmente satisfeitas, enquanto que de $f_{13}=0, f_{14}=0$, $f_{23}=0$ e $f_{24}=0$ resultam $m_{2}=m_{4}, m_{2}=m_{3}, m_{1}=m_{4}$ e $m_{1}=m_{3}$, respectivamente. Portanto, $m_{1}=m_{2}=m_{3}=m_{4}$ e o item 3 do Teorema 5.3.1 está demonstrado.

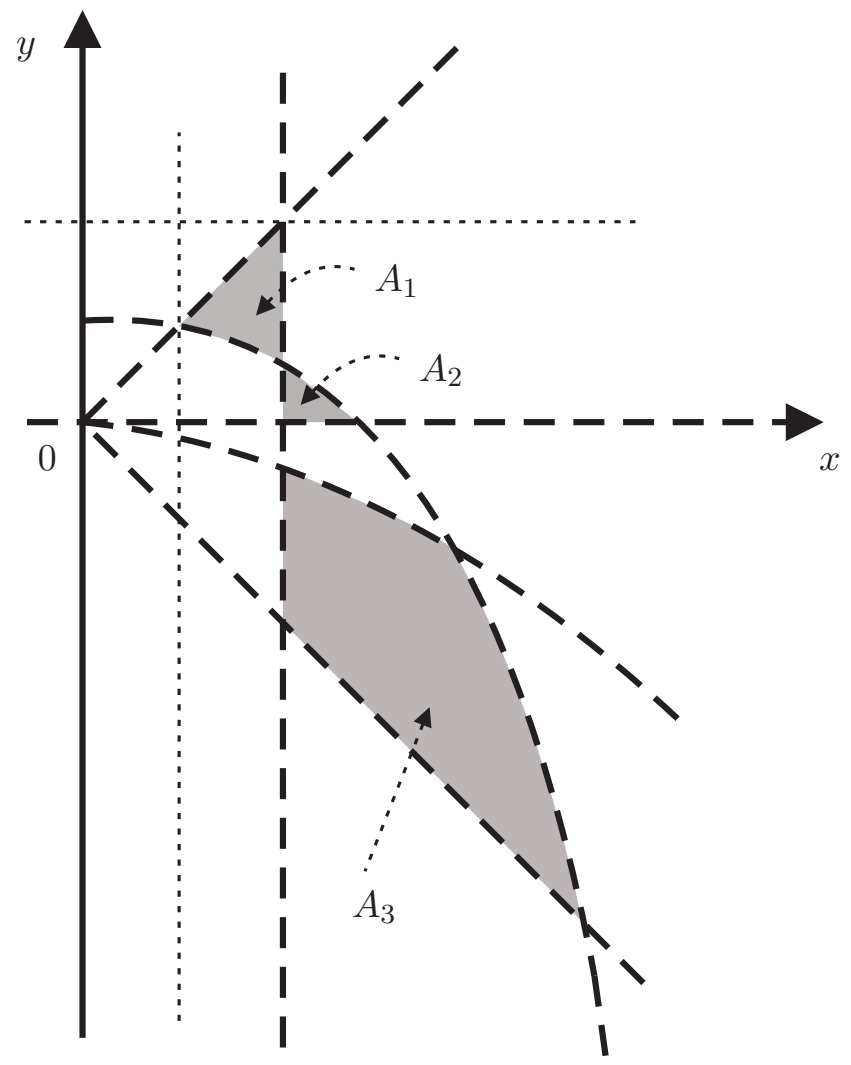

Figura 5.10: Regiões abertas $A_{1}, A_{2}$ e $A_{3}$.

Baseados no Teorema 5.3.1, seguem os seguintes corolários.

Corolário 5.3.2 Para cada $1 / 2<x_{0}<\sqrt{3} / 2$, existem dois intervalos abertos disjuntos $I_{x_{0}}^{+}$e $I_{x_{0}}^{-}$, tal que para cada $y_{0} \in I_{x_{0}}^{+} \cup I_{x_{0}}^{-}$, existem massas positivas $m_{1}=m_{2}, m_{3}$ e $m_{4}$, de modo que os quatro 
corpos, como na figura 5.7, estejam numa configuração central do tipo pipa. Mais especificamente, se $y_{0} \in I_{x_{0}}^{+}$a configuração central é do tipo pipa côncava e se $y_{0} \in I_{x_{0}}^{-}$a configuração central é do tipo pipa convexa.

Corolário 5.3.3 Considere $0<x<(2 \sqrt{3}-3) / 2$. Então não existem $y \in \mathbb{R}$ e massas positivas $m_{1}, m_{2}, m_{3}$ e $m_{4}$, de modo que os quatro corpos, como na figura 5.7, estejam numa configuração central do tipo pipa.

Corolário 5.3.4 Considere $y<\sqrt{3} / 2$. Então, se $x>(2 \sqrt{3}+3) / 2$, não existem massas positivas $m_{1}, m_{2}, m_{3}$ e $m_{4}$, de modo que os quatro corpos, como na figura 5.7, estejam numa configuração central do tipo pipa.

Como no caso da configuração equilátera de Lagrange, tínhamos um análogo espacial, podemos nos perguntar se no caso das pipas isto também ocorre? A resposta é sim e pode ser vista no teorema seguinte. Aplicaremos as equações de Andoyer com volumes (5.4). O próximo resultado pode ser encontrado em [25].

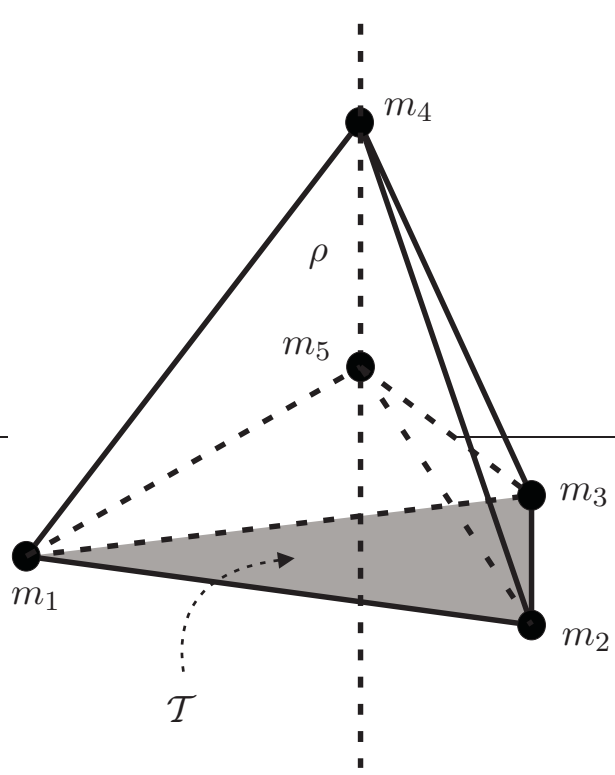

(a)

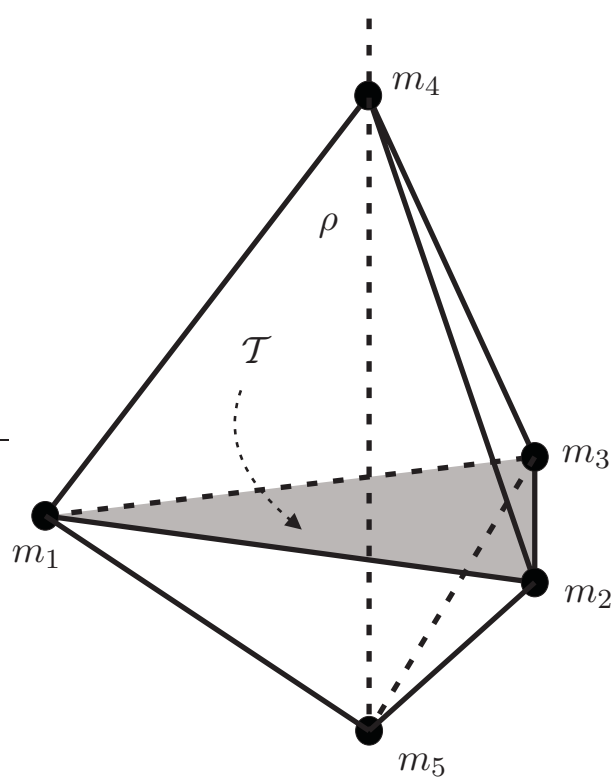

(b)

Figura 5.11: Configurações centrais espaciais para 5-corpos. Configuração côncava (a). Configuração convexa (b). 
Consideremos um problema de 5 corpos que de certa forma é uma generalização para o caso da pipa acima. Vamos supor as seguintes condições, vide figuras 5.11(a) e 5.11(b):

1. $\mathrm{O}$ vetores posição $r_{1}, r_{2}$ e $r_{3}$ estão nos vértices de um triângulo equilátero $\mathcal{T}$;

2. Defina a reta $\rho$ passando pelo baricentro de $\mathcal{T}$ perpendicular ao plano que contém $\mathcal{T}$. O vetor $r_{4} \in \rho$ está fixo e não pertence ao plano contendo $\mathcal{T}$;

3. $\mathrm{O}$ vetor $r_{5} \in \rho, r_{5} \neq r_{4}$.

Este tipo de configuração é chamada côncava se um dos corpos está no interior do fecho convexo dos outros quatro, vide figura 5.11(a). Caso contrário a configuração é chamada convexa, vide figura 5.11(b). Aqui diremos que uma configuração é côncava do tipo 1 se o corpo 5 está no interior do fecho convexo de 1, 2, 3 e 4, e diremos que a configuração é côncava do tipo 2 se o corpo 4 está no interior do fecho convexo de $1,2,3,5$.

Para simplificar a notação em nossos resultados, vamos considerar as seguintes coordenadas no nosso problema, $r_{1}=(x, 0,0), r_{2}=(-x / 2,-\sqrt{3} x / 2,0), r_{3}=(-x / 2, \sqrt{3} x / 2,0), r_{4}=(0,0, \sqrt{6} / 3)$ e $r_{5}=(0,0, y) \operatorname{com} x>0$ e $y \neq \sqrt{6} / 3$, vide figura 5.12. Assim, $x$ é o raio da circunferência circunscrita ao triângulo $\mathcal{T}$ e $y$ é a altura, com sinal, do quinto corpo com respeito ao plano que contém $\mathcal{T}$. Note que o quinto corpo estaria no baricentro de $\mathcal{T}$ se $y=0$. Por conseguinte, a configuração é convexa se, e somente se, $y \leq 0$.

Com estas considerações temos o seguinte resultado.

Teorema 5.3.5 Considere vetores posição nos vértices de um triângulo equilátero $\mathcal{T}, r_{1}=(x, 0,0)$, $r_{2}=(-x / 2,-\sqrt{3} x / 2,0), r_{3}=(-x / 2, \sqrt{3} x / 2,0)$ e vetores posição $r_{4}=(0,0, \sqrt{6} / 3), r_{5}=(0,0, y)$ sobre $\rho$, onde $\rho$ é a reta que passa pelo baricentro de $\mathcal{T}$ perpendicular ao plano que contém $\mathcal{T}$, de acordo com a figura 5.12. Assim, as seguintes afirmações são válidas:

1. Existe um valor minimo $x=x_{\text {min }}=(3 \sqrt{2}-2 \sqrt{3}) / 3$, tal que se $0<x \leq x_{\text {min }}$ não existem posições $r_{1}, \ldots, r_{5}$ nem massas positivas $m_{1}, \ldots, m_{5}$, tais que estes corpos formem uma configuração central; 


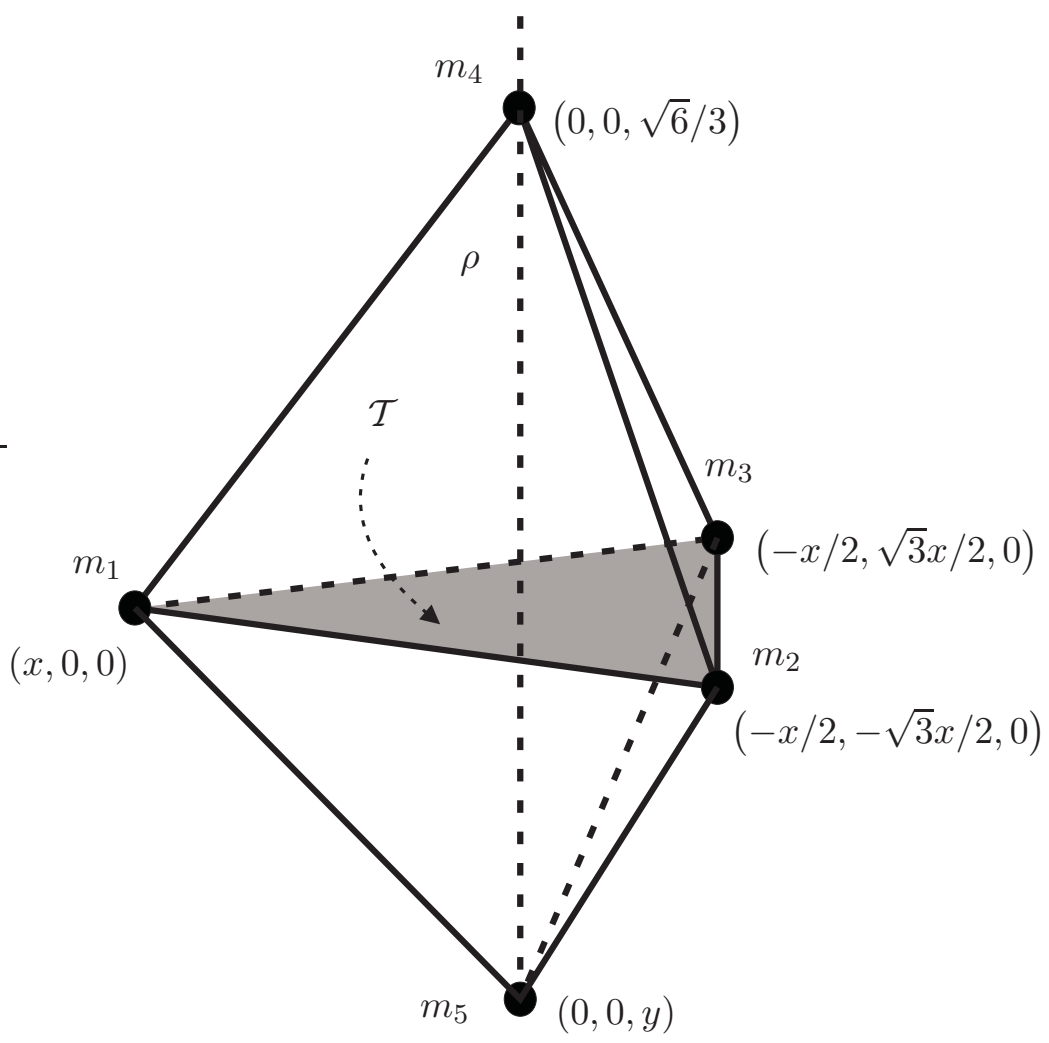

Figura 5.12: Coordenadas para o problema com 5 corpos.

2. Existem dois segmentos $I_{1}=((3 \sqrt{2}-2 \sqrt{3}) / 3, \sqrt{3} / 3)$ e $I_{2}=(\sqrt{3} / 3, \sqrt{6} / 3)$, tais que para cada $x \in I_{1} \cup I_{2}$ existe um segmento não vazio de possíveis valores para $y$, portanto possíveis posições para $r_{5}$ e massas positivas $m_{1}, \ldots, m_{5}$, tais que estes corpos formam uma família a um parâmetro de configurações centrais côncavas do tipo 1;

3. Existe um valor distinguido e bem determinado $x=\bar{x}$ para o qual existe apenas uma posição $r_{5}$, tal que os corpos formam uma familia dois parâmetros de configurações centrais côncavas do tipo 1. Em verdade, esta configuração é exatamente aquela em que temos quatro massas iguais nos vértices de um tetraedro regular e o quinto corpo no baricentro deste tetraedro;

4. Existe um segmento $I_{3}=(\sqrt{3} / 3,(2 \sqrt{3}+3 \sqrt{2}) / 3)$, tal que para cada $x \in I_{3}$ existe um segmento não vazio de possíveis valores para $y$, portanto possíveis posições para $r_{5}$ e massas positivas $m_{1}, \ldots, m_{5}$, tais que estes corpos formam uma familia a um parâmetro de configurações centrais convexas; 
5. Existe um segmento ilimitado à direita $I_{4}=(4 \sqrt{3} / 3,+\infty)$, tal que para cada $x \in I_{4}$ existe um segmento não vazio de possiveis valores para $y$, portanto possíveis posições para $r_{5} e$ massas positivas $m_{1}, \ldots, m_{5}$, tais que estes corpos formam uma família a um parâmetro de configurações centrais côncavas do tipo 2;

6. Existe um valor distinguido e bem determinado $x=\bar{x}$ para o qual existe apenas uma posição $r_{5}$, tal que os corpos formam uma família dois parâmetros de configurações centrais côncavas do tipo 2. Em verdade, esta configuração é exatamente aquela em que temos quatro massas iguais nos vértices de um tetraedro regular e o quinto corpo no baricentro deste tetraedro.

Observação 5.3.6 Neste resultado os itens 2, 3 e 4 do teorema estão relacionados aos resultados obtidos por Leandro em [19], assim como os itens 3 e 6 podem ser encontrados em [47].

Prova: Usaremos as equações de Andoyer com volumes (5.4). Para cinco corpos teremos um sistema de 30 equações, mas existem algumas simetrias no nosso caso que simplificam os cálculos. Para as distâncias entre os corpos temos $R_{12}=R_{13}=R_{23}, R_{14}=R_{24}=R_{34}$ e $R_{15}=R_{25}=R_{35}$. Com nosso sistema de coordenadas temos $\Delta_{1234}>0$. Levando em conta as simetrias nos vem

$$
\begin{aligned}
& \Delta_{1425}=-\Delta_{1435}=-\Delta_{2415}=\Delta_{2435}=\Delta_{3415}=-\Delta_{3425}, \\
& \Delta_{1523}=-\Delta_{1532}=-\Delta_{2513}=\Delta_{2531}=\Delta_{3512}=-\Delta_{3521}, \\
& \Delta_{1524}=-\Delta_{1534}=-\Delta_{2514}=\Delta_{2534}=\Delta_{3514}=-\Delta_{3524} .
\end{aligned}
$$

Levando estas simetrias nas equações de Andoyer temos 9 equações de (5.4) trivialmente satisfeitas $f_{123}=0, f_{124}=0, f_{125}=0, f_{132}=0, f_{134}=0, f_{135}=0, f_{231}=0, f_{234}=0$ e $f_{235}=0$.

Ainda notando as simetrias, as seguintes equivalências entre equações são válidas,

$$
\begin{aligned}
& f_{145}=0 \Leftrightarrow f_{154}=0 \Leftrightarrow f_{451}=0 \Leftrightarrow\left(R_{12}-R_{24}\right) \Delta_{1452}\left(m_{2}-m_{3}\right)=0, \\
& f_{245}=0 \Leftrightarrow f_{254}=0 \Leftrightarrow f_{452}=0 \Leftrightarrow\left(R_{12}-R_{14}\right) \Delta_{2451}\left(m_{1}-m_{3}\right)=0,
\end{aligned}
$$




$$
f_{345}=0 \Leftrightarrow f_{354}=0 \Leftrightarrow f_{453}=0 \Leftrightarrow\left(R_{13}-R_{14}\right) \Delta_{3451}\left(m_{1}-m_{2}\right)=0 .
$$

Daí as 21 equações restantes de (5.4) são reduzidas a 15 equações. Como nossas classes de configurações centrais satisfazem $r_{4} \neq r_{5}$ temos $\Delta_{1452} \neq 0, \Delta_{2451} \neq 0$ e $\Delta_{3451} \neq 0$. Das equações (5.23), (5.24) e (5.25) temos que analisar dois casos: quando as massas $m_{1}=m_{2}=m_{3}$ e quando $r_{1}, r_{2}, r_{3}$ e $r_{4}$ estão nos vértices de um tetraedro regular.

Caso 1. Considere $r_{1}, r_{2}, r_{3}$ e $r_{4}$ nos vértices de um tetraedro regular. Temos então o seguinte lema.

Lema 5.3.7 Considere $r_{1}, r_{2}, r_{3}$ e $r_{4}$ nos vértices de um tetraedro regular. Então, existe apenas uma posição $r_{5}$ no centro de massa do tetraedro e massas positivas $m_{1}=m_{2}=m_{3}=m_{4}=m e$ $m_{5}$, tais que os corpos formam uma família a dois parâmetros de configurações centrais côncavas do tipo 1 .

Prova. Devido às simetrias as seguintes equações são equivalentes em (5.4)

$$
\begin{aligned}
& f_{142}=0 \Leftrightarrow f_{241}=0, \quad f_{143}=0 \Leftrightarrow f_{341}=0, \quad f_{243}=0 \Leftrightarrow f_{342}=0, \\
& f_{152}=0 \Leftrightarrow f_{251}=0, \quad f_{153}=0 \Leftrightarrow f_{351}=0, \quad f_{253}=0 \Leftrightarrow f_{352}=0 .
\end{aligned}
$$

Daí as 15 equações restantes são reduzidas a 9 equações. De $f_{142}=0, f_{143}=0,2 f_{243}=0$ temos $m_{5}\left(R_{15}-R_{45}\right) \Delta_{1425}=0, m_{5}\left(R_{15}-R_{45}\right) \Delta_{1435}=0$ e $m_{5}\left(R_{25}-R_{45}\right) \Delta_{2435}=0$, respectivamente. As $\Delta_{1425} \neq 0, \Delta_{1435} \neq 0$ e $\Delta_{2435} \neq 0$ segue que $R_{15}=R_{45}=R_{25}$. Isto implica que $r_{5}$ deve estar no centro de massa do tetraedro. Levando esta informação em $f_{152}=0, f_{153}=0$ e $f_{253}=0$ temos $\left(m_{3}-m_{4}\right)\left(R_{13}-R_{35}\right) \Delta_{1523}=0,\left(m_{2}-m_{4}\right)\left(R_{12}-R_{25}\right) \Delta_{1532}=0$ e $\left(m_{1}-m_{4}\right)\left(R_{12}-R_{15}\right) \Delta_{2531}=$ 0 , respectivamente. Estas 3 últimas equações são satisfeitas somente quando $m_{1}=m_{2}=m_{3}=m_{4}$. Por sua vez $m_{5}$ pode assumir qualquer valor positivo. As outras 3 equações restantes $f_{145}=0$, $f_{245}=0$ e $f_{345}=0$ são trivialmente verificadas. Assim o lema está provado.

Defina $\bar{x}=\sqrt{3} / 3 . \quad$ Assim $r_{1}=(\sqrt{3} / 3,0,0), r_{2}=(-\sqrt{3} / 6,-1 / 2,0), r_{3}=(-\sqrt{3} / 6,1 / 2,0)$, 
$r_{4}=(0,0, \sqrt{6} / 3)$ e $r_{5}=(0,0, \sqrt{6} / 12)$. O Lema 5.3.7 prova item 3 do Teorema 5.3.5. No restante desta prova omitiremos esta situação de configuração central côncava.

Caso 2. Considere $m_{1}=m_{2}=m_{3}=m$. Então as equações (5.23), (5.24) e (5.25) são satisfeitas e

$$
\begin{aligned}
& f_{142}=0 \Leftrightarrow f_{143}=0 \Leftrightarrow f_{241}=0 \Leftrightarrow f_{243}=0 \Leftrightarrow f_{341}=0 \Leftrightarrow f_{342}=0, \\
& f_{152}=0 \Leftrightarrow f_{153}=0 \Leftrightarrow f_{251}=0 \Leftrightarrow f_{253}=0 \Leftrightarrow f_{351}=0 \Leftrightarrow f_{352}=0 .
\end{aligned}
$$

Noutros termos, as 30 equações foram reduzidas a apenas 2, as quais seguem

$$
\begin{aligned}
& f_{142}=m\left(R_{13}-R_{34}\right) \Delta_{1423}+m_{5}\left(R_{15}-R_{45}\right) \Delta_{1425}=0, \\
& f_{152}=m\left(R_{13}-R_{35}\right) \Delta_{1523}+m_{4}\left(R_{14}-R_{45}\right) \Delta_{1524}=0 .
\end{aligned}
$$

As equações (5.26) e (5.27) podem ser resolvidas diretamente de forma a termos $m_{4}=m_{4}(x, y, m)$ e $m_{5}=m_{5}(x, y, m)$, respectivamente. Nestas equações $m$ pode ser entendido como um parâmetro para as configurações centrais.

Da equação (5.26) e (5.27) temos

$$
\begin{aligned}
& \frac{m_{4}}{m}=\frac{\left(R_{35}-R_{13}\right) \Delta_{1523}}{\left(R_{14}-R_{45}\right) \Delta_{1524}}, \\
& \frac{m_{5}}{m}=\frac{\left(R_{34}-R_{13}\right) \Delta_{1423}}{\left(R_{15}-R_{45}\right) \Delta_{1425}} .
\end{aligned}
$$

O que precisamos determinar são os subconjuntos de $\mathcal{D}=\{x>0, y \in \mathbb{R}, y \neq \sqrt{6} / 3\}$ onde ambas as razões de massas $m_{4} / m$ e $m_{5} / m$ são positivas. Para o estudo dos sinais os termos que aparecem nas expressões (5.28) e (5.29) temos $R_{35}-R_{13}=0$ se e só se $(x, y) \in\{x>0, y=-\sqrt{2} x\} \cup\{x>$ $0, y=\sqrt{2} x\}$ (Retas), $R_{14}-R_{45}=0$ se e só se $(x, y) \in\left\{x>0, y=\sqrt{6} / 3-\sqrt{9 x^{2}+6} / 3\right\} \cup\{x>$ $\left.0, y=\sqrt{6} / 3+\sqrt{9 x^{2}+6} / 3\right\}$ (Ramo de Hipérbole), $R_{34}-R_{13}=0$ se e só $\operatorname{se}(x, y) \in\{x=\sqrt{3} / 3\}$, $R_{15}-R_{45}=0$ se e só se $(x, y) \in\left\{x>0, y=\sqrt{6}\left(2-3 x^{2}\right) / 12\right\}$ (Parábola), $\Delta_{1523}=0$ se e só se $(x, y) \in\{x>0, y=0\}, \Delta_{1524}=0$ se e só se $(x, y) \in\{x>0, y=\sqrt{6} / 3\}, \Delta_{1425}=0$ se e só se 
$(x, y) \in\{x>0, y=\sqrt{6} / 3\}$. Veja figuras 5.13 e 5.14 .

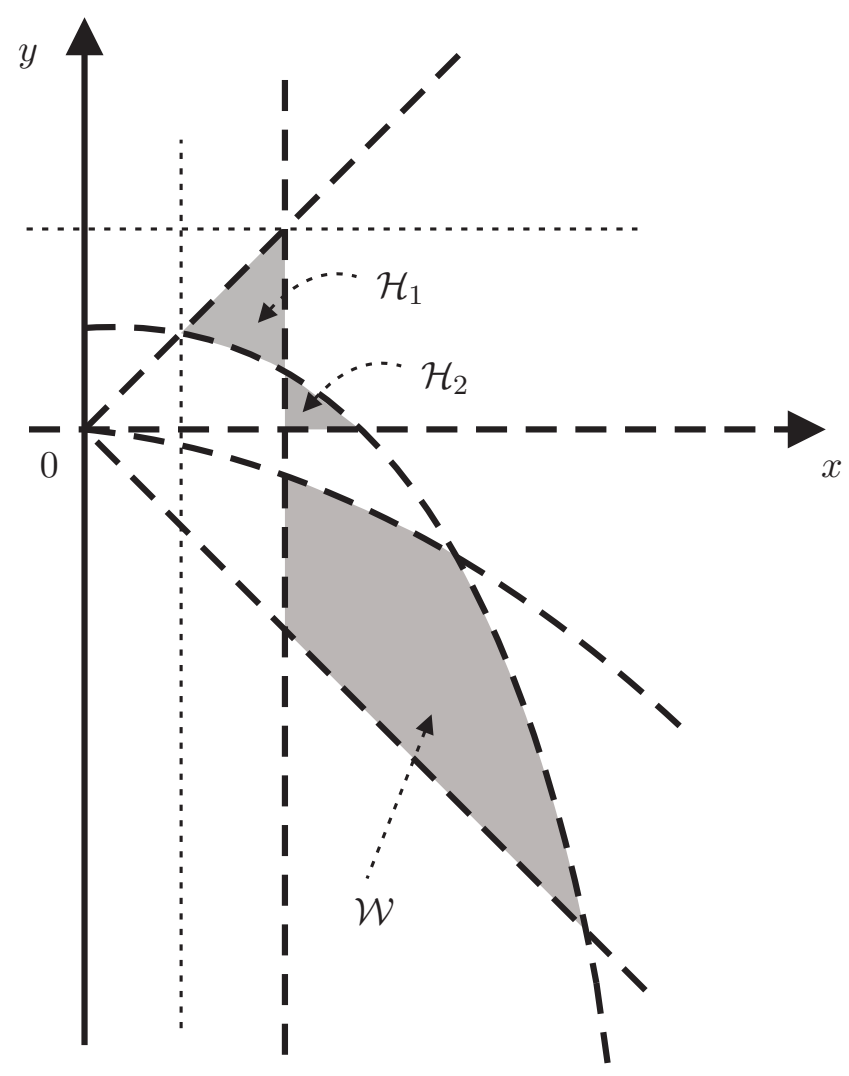

Figura 5.13: Regiões abertas $\mathcal{H}_{1}, \mathcal{H}_{2}$ e $\mathcal{W}$.

Caso 2.1. Considere $x>0$ e $y=0$.

Lema 5.3.8 Considere $r_{5}=(0,0,0)$, que é $y=0$. Então, não existe $x>0$ tal que os 5 corpos com massas positivas formem uma configuração central de acordo com a figura 5.12.

Prova. Das equações (5.27) temos

$$
m\left(R_{13}-R_{35}\right) \Delta_{1523}+m_{4}\left(R_{14}-R_{45}\right) \Delta_{1524}=0 .
$$

Por hipótese $\Delta_{1523}=0$. Como $\left(R_{14}-R_{45}\right) \neq 0$ e $\Delta_{1524} \neq 0$ então $m_{4}=0$. Isto é uma contradição.

Caso 2.2. Considere $0<x \leq(3 \sqrt{2}-2 \sqrt{3}) / 3$ e $y \neq \sqrt{6} / 3$. Temos o seguinte lema. 
Lema 5.3.9 Considere $0<x \leq(3 \sqrt{2}-2 \sqrt{3}) / 3$. Então não existe posição para o corpo 5 sobre $\rho$ e massas positivas $m_{i}, i=1, \ldots, 5$, tais que estes corpos formem uma configuração central de acordo com a figura 5.12.

Prova. Devemos analisar três casos: $y<0,0<y<\sqrt{6} / 3$ e $y>\sqrt{6} / 3$.

Considere $y<0$. Da equação (5.26) temos

$$
m\left(R_{13}-R_{34}\right) \Delta_{1423}+m_{5}\left(R_{15}-R_{45}\right) \Delta_{1425}=0 .
$$

Por hipótese $R_{13}-R_{34}<0, R_{15}-R_{45}<0, \Delta_{1423}>0$ e $\Delta_{1425}>0$. Por conseguinte, os coeficientes da expressão acima tem o mesmo sinal. Para que se anule precisamos que $m_{5}$ e $m$ tenham sinais opostos. Isto é uma contradição.

Considere $0<y<\sqrt{6} / 3$. Por hipótese $R_{13}-R_{34}<0, R_{13}-R_{35}>0, R_{14}-R_{45}<0, \Delta_{1423}>0$, $\Delta_{1425}>0, \Delta_{1523}>0, \Delta_{1524}<0, R_{15}-R_{45}<0$, se $0<y<\sqrt{6}\left(2-3 x^{2}\right) / 12$ e $R_{15}-R_{45}>0$, se $\sqrt{6}\left(2-3 x^{2}\right) / 12<y<\sqrt{6} / 3$.

Da equação (5.26) temos

$$
m\left(R_{13}-R_{34}\right) \Delta_{1423}+m_{5}\left(R_{15}-R_{45}\right) \Delta_{1425}=0 .
$$

Se $0<y<\sqrt{6}\left(2-3 x^{2}\right) / 12$ então os coeficientes da expressão acima acima têm o mesmo sinal, portanto para que se anule é preciso que $m_{5}$ e $m$ tenham sinais opostos. Isto é uma contradição.

Considere agora a equação (5.27) temos

$$
m\left(R_{13}-R_{35}\right) \Delta_{1523}+m_{4}\left(R_{14}-R_{45}\right) \Delta_{1524}=0 .
$$

Se $\sqrt{6}\left(2-3 x^{2}\right) / 12<y<\sqrt{6} / 3$ então os coeficientes da expressão acima acima têm o mesmo sinal, portanto para que se anule é preciso que $m_{4}$ e $m$ tenham sinais opostos. Isto é uma contradição. Se $y=\sqrt{6}\left(2-3 x^{2}\right) / 12$ então $R_{15}-R_{45}=0$ mas isto implica que $m=0$ na equação (5.26). Novamente temos contradição. 
Considere $y>\sqrt{6} / 3$. Da equação (5.26) temos

$$
m\left(R_{13}-R_{34}\right) \Delta_{1423}+m_{5}\left(R_{15}-R_{45}\right) \Delta_{1425}=0 .
$$

Por hipótese $R_{13}-R_{34}<0, R_{15}-R_{45}>0, \Delta_{1423}>0$ e $\Delta_{1425}<0$. Por conseguinte os coeficientes da expressão acima acima têm o mesmo sinal, portanto para que se anule é preciso que $m_{5}$ e $m$ tenham sinais opostos. Isto é uma contradição.

Do Lema 5.3.9 provamos o item 1 do Teorema 5.3.5. O valor $x_{\text {min }}=(3 \sqrt{2}-2 \sqrt{3}) / 3$ é definido como a coordenada $x$ da interseção das curvas $R_{35}-R_{13}=0$ e $R_{15}-R_{45}=0$, vide figura 5.13.

Caso 2.3. Considere $x>0$ e $0<y<\sqrt{6} / 3$.

Defina $\mathcal{H}=\mathcal{H}_{1} \cup \mathcal{H}_{2}$, vide figura 5.13,onde

$$
\begin{gathered}
\mathcal{H}_{1}=\left\{\frac{3 \sqrt{2}-2 \sqrt{3}}{3}<x<\frac{\sqrt{3}}{3}, \frac{\sqrt{6}\left(2-3 x^{2}\right)}{12}<y<\sqrt{2} x\right\}, \\
\mathcal{H}_{2}=\left\{\frac{\sqrt{3}}{3}<x<\frac{\sqrt{6}}{3}, 0<y<\frac{\sqrt{6}\left(2-3 x^{2}\right)}{12}\right\} .
\end{gathered}
$$

Para $(x, y) \in \mathcal{H}_{1}$ temos $x>0,0<y<\sqrt{6} / 3, R_{35}-R_{13}>0, R_{34}-R_{13}<0, R_{15}-R_{45}<0$, $R_{14}-R_{45}<0, \Delta_{1523}>0, \Delta_{1524}<0, \Delta_{1423}>0$ e $\Delta_{1425}>0$. Para $(x, y) \in \mathcal{H}_{2}$ temos $x>0$, $0<y<\sqrt{6} / 3, R_{35}-R_{13}>0, R_{34}-R_{13}>0, R_{15}-R_{45}>0, R_{14}-R_{45}<0, \Delta_{1523}>0, \Delta_{1524}<0$, $\Delta_{1423}>0$ e $\Delta_{1425}>0$.

É fácil ver que para $(x, y) \in \mathcal{H}$ o lado direito das expressões (5.28) e (5.29) é positivo, portanto temos configurações centrais côncavas do tipo 1.

As projeções ortogonais dos conjuntos abertos $\mathcal{H}_{1}$ e $\mathcal{H}_{2}$ sobre o eixo $x$ nos dá dois segmentos $I_{1}=((3 \sqrt{2}-2 \sqrt{3}) / 3, \sqrt{3} / 3)$ e $I_{2}=(\sqrt{3} / 3, \sqrt{6} / 3)$, respectivamente. Para cada $x_{*} \in I_{1} \cup I_{2}$ a reta $x=x_{*}$ intersecta $\mathcal{H}_{1} \cup \mathcal{H}_{2}$ em um segmento não vazio. Isto prova o item 2 do Teorema 5.3.5.

Caso 2.4. Considere $x>0$ e $y<0$.

Para termos configurações centrais convexas é necessário que $y<0$. Defina o conjunto aberto 
$\mathcal{W}=\mathcal{W}_{1} \cup \mathcal{W}_{2}$, vide figura 5.13 , onde

$$
\begin{gathered}
\mathcal{W}_{1}=\left\{\frac{\sqrt{3}}{3}<x \leq \sqrt{2},-\sqrt{2} x<y<\frac{\sqrt{6}}{3}-\frac{\sqrt{9 x^{2}+6}}{3}\right\}, \\
\mathcal{W}_{2}=\left\{\sqrt{2} \leq x<\frac{2 \sqrt{3}+3 \sqrt{2}}{3},-\sqrt{2} x<y<\frac{\sqrt{6}\left(2-3 x^{2}\right)}{12}\right\} .
\end{gathered}
$$

Para $(x, y) \in \mathcal{W}$ temos $x>0, y<0, R_{35}-R_{13}>0, R_{34}-R_{13}>0, R_{15}-R_{45}>0, R_{14}-R_{45}>0$, $\Delta_{1523}<0, \Delta_{1524}<0, \Delta_{1423}>0$ e $\Delta_{1425}>0$.

É fácil ver que para $(x, y) \in \mathcal{W}$ o lado direito das expressões (5.28) e (5.29) é positivo e por conseguinte temos configurações centrais convexas.

A projeção ortogonal de $\mathcal{W}$ sobre o eixo $x$ nos dá um segmento $I_{3}=(\sqrt{3} / 3,(2 \sqrt{3}+3 \sqrt{2}) / 3)$. Para cada $x_{*} \in I_{3}$ a reta $x=x_{*}$ intersecta $\mathcal{W}$ em um segmento não vazio. Isto prova o item 4 do Teorema 5.3.5.

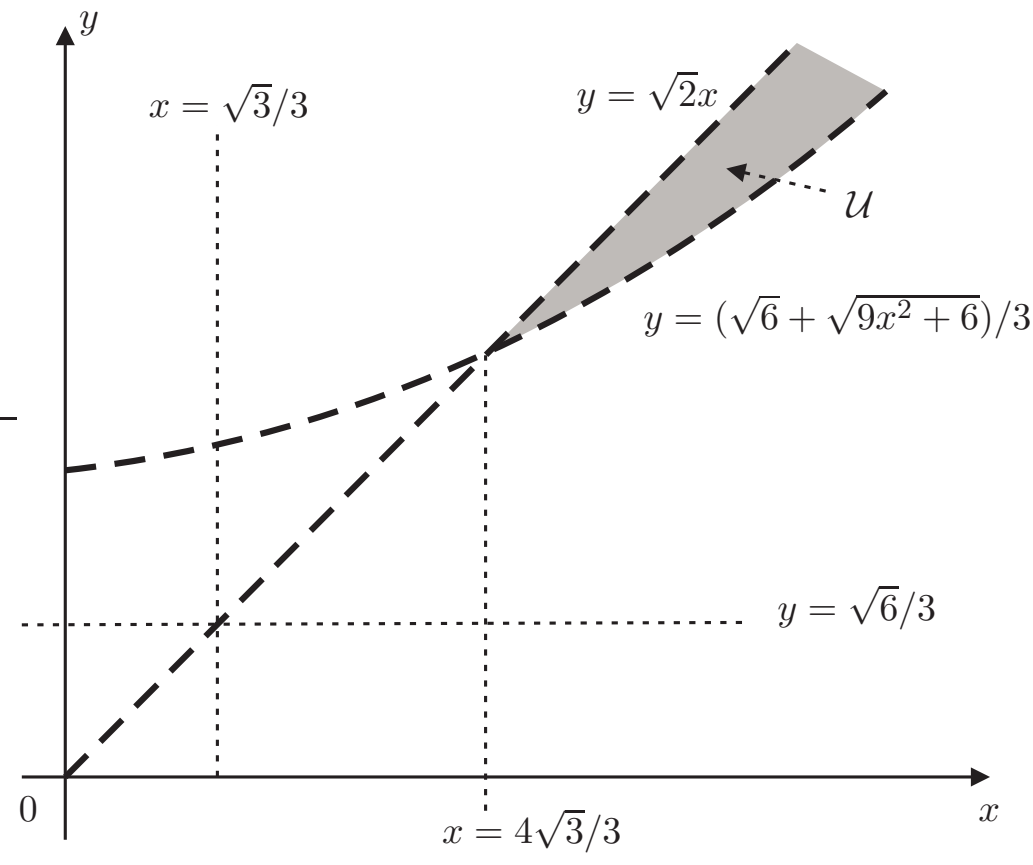

Figura 5.14: Região aberta $\mathcal{U}$.

Caso 2.5. Considere $x>0$ e $y>\sqrt{6} / 3$. 
Defina seguinte região ilimitada aberta, vide figura 5.14,

$$
\mathcal{U}=\left\{\frac{4 \sqrt{3}}{3}<x, \frac{\sqrt{6}+\sqrt{9 x^{2}+6}}{3}<y<\sqrt{2} x\right\}
$$

Para $(x, y) \in \mathcal{U}$ temos $x>0, y>\sqrt{6} / 3, R_{35}-R_{13}>0, R_{34}-R_{13}>0, R_{15}-R_{45}<0, R_{14}-R_{45}>0$, $\Delta_{1523}>0, \Delta_{1524}>0, \Delta_{1423}>0$ e $\Delta_{1425}<0$.

É fácil ver que para $(x, y) \in \mathcal{U}$ o lado direito das expressões (5.28) e (5.29) é positivo e por conseguinte temos configurações centrais côncavas do tipo 2 .

A projeção ortogonal de $\mathcal{U}$ sobre o eixo $x$ nos dá o segmento ilimitado $I_{4}=(4 \sqrt{3} / 3,+\infty)$. Para cada $x_{*} \in I_{4}$ a reta $x=x_{*}$ intersecta $\mathcal{U}$ em um segmento não vazio. Isto prova o item 5 do Teorema 5.3.5.

Caso 2.6. Considere $r_{1}, r_{2}, r_{3}$ e $r_{5}$ nos vértices de um tetraedro regular. Temos o seguinte lema.

Lema 5.3.10 Se $m_{1}=m_{2}=m_{3}=m_{5}=m$ e $m_{4}$ estão em $r_{1}=(4 \sqrt{3} / 3,0,0), r_{2}=(-2 \sqrt{3} / 3,-2,0)$, $r_{3}=(-2 \sqrt{3} / 3,2,0), r_{4}=(0,0, \sqrt{6} / 3)$ e $r_{5}=(0,0,4 \sqrt{6} / 3)$ então estes corpos formam uma família a dois parâmetros de configurações centrais côncavas do tipo 2.

Prova. Por hipótese $x=4 \sqrt{3} / 3$ e $y=4 \sqrt{6} / 3$. Para estes valores temos $R_{13}-R_{35}=0$ e $R_{14}-R_{45}=0$. Por conseguinte, a expressão (5.27) é satisfeita para todo $m_{4}>0$. Como $m_{5}=m$ a equação (5.26) pode ser escrita como

$$
m\left[\left(R_{13}-R_{34}\right) \Delta_{1423}+\left(R_{15}-R_{45}\right) \Delta_{1425}\right]=0 .
$$

Com estes valores para $x$ e $y$ temos $r_{13}=r_{15}=4, r_{34}=r_{45}=\sqrt{6}, \Delta_{1423}=-\Delta_{1425}=8 \sqrt{2}$. Por um cálculo direto vemos que $\left(R_{13}-R_{34}\right) \Delta_{1423}+\left(R_{15}-R_{45}\right) \Delta_{1425}=0$. É fácil ver que $r_{1}, r_{2}, r_{3} \mathrm{e}$ $r_{5}$ estão nos vértices de um tetraedro regular e que $r_{4}$ está no baricentro do tetraedro.

A prova do item 6 do Teorema 5.3.5 segue do Lema 5.3.10 onde $\overline{\bar{x}}=4 \sqrt{3} / 3$. Isto finda a prova 
do teorema.

Observando as provas dos Teoremas 5.3.1 e 5.3.5 é notável a semelhança entre as figuras 5.10 e 5.13. De fato, no problema planar também temos uma região ilimitada semelhante àquela mostrada na figura 5.14. Uma pergunta natural é: Substituindo o triângulo equilátero da base por um outro polígono regular o resultado é, ainda, similar? Se a resposta for afirmativa temos de encontrar uma prova simples para a mesma.

\subsection{Configurações Centrais Empilhadas Parte 1}

Um caso interessante de configurações centrais foi proposto por Hampton em [13]. Neste trabalho Hampton estudou configurações centrais com cinco massas, nas quais três massas estão sempre nos vértices de um triângulo equilátero e outras duas massas no interior do triângulo simetricamente colocadas em relação a uma mediatriz do triângulo, vide figura 5.15.

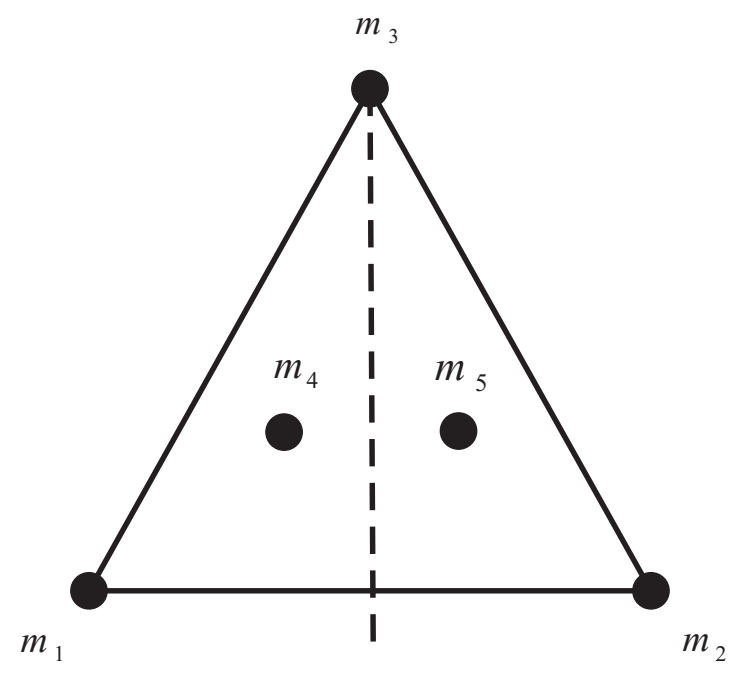

Figura 5.15: Configuração de Hampton.

O resultado de Hampton segue no seguinte teorema, a prova pode ser vista em [13].

Teorema 5.4.1 Considere uma configuração como a mostrada na figura 5.15. Então existem 
massas positivas

$$
\left(m_{1}, m_{2}, m_{3}, m_{4}, m_{5}\right)=(1,1, M, m, m)
$$

posições

$$
r_{12}=r_{23}=r_{13}=1, \quad \text { onde } r_{i j}=\left|r_{i}-r_{j}\right|
$$

$e$

$$
r_{14}=r_{25}<r_{34}=r_{35}<r_{24}=r_{15}<1
$$

as quais formam uma configuração central. Em verdade, temos uma família a um parâmetro de configurações centrais, de forma que existe uma curva no plano, simétrica com relação à mediatriz do triângulo e conectando $r_{1}$ a $r_{2}$, onde podemos colocar $m_{4}$ e $m_{5}$ de modo que as cinco massas formem uma configuração central, conforme a figura 5.16

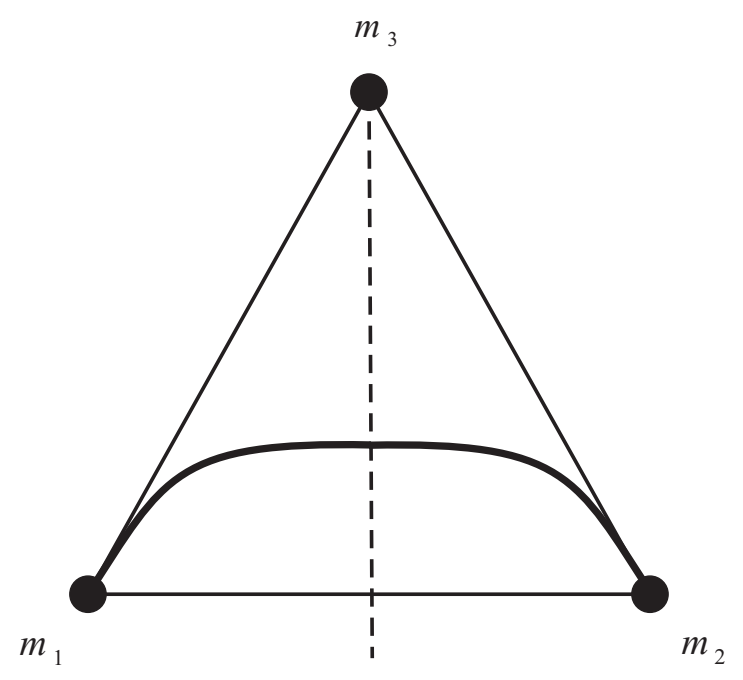

Figura 5.16: Curva de configurações centrais exemplo de Hampton.

Observando a figura 5.16 podemos destacar a seguinte propriedade. Existe um subconjunto das 5 massas a saber, as massas sobre os vértices do triângulo equilátero, que formam uma configuração central. De certa forma, é possível retirar as duas massas $m_{4}$ e $m_{5}$ que ainda teremos uma configuração central. Tais configurações são chamadas de configurações empilhadas, configurações centrais nas quais podemos retirar algumas massas e ainda teremos uma configuração central. Uma 
definição mais precisa será vista mais adiante nesta seção.

Novamente podemos nos perguntar se existe um análogo espacial deste caso? A resposta é sim e a resposta e também é devida a Hampton e Santoprete. Em [14] é estudado o caso onde temos quatro massas nos vértices de um tetraedro regular e três massas nos vértices de um triângulo equilátero paralelo a base do tetraedro e no interior do tetraedro, conforme figura 5.17.

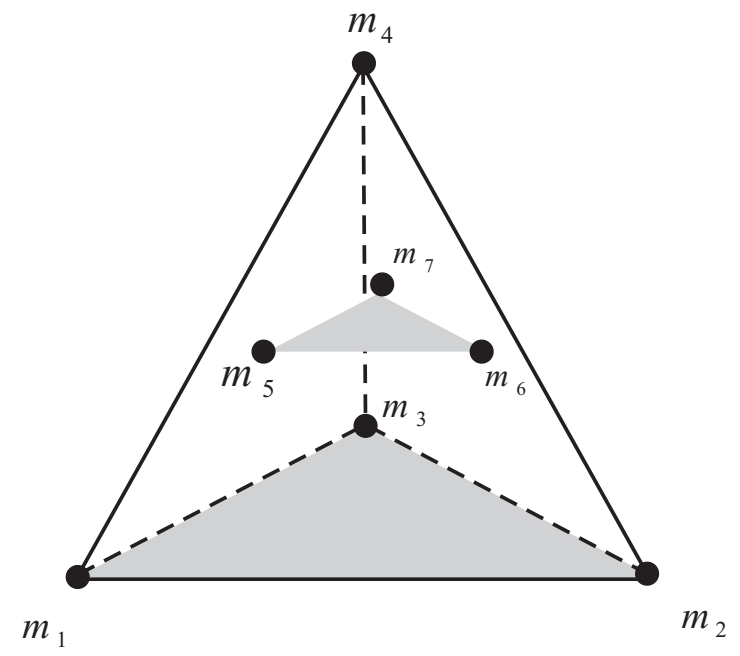

Figura 5.17: Configuração espacial de Hampton e Santoprete.

O resultado de Hampton e Santoprete segue no seguinte teorema, a prova pode ser vista em [14].

Teorema 5.4.2 Considere uma configuração como a mostrada na figura 5.17, então existem massas positivas

$$
\left(m_{1}, m_{2}, m_{3}, m_{4}, m_{5}, m_{6}, m_{7}\right)=(1,1,1, M, m, m, m)
$$

posições

$$
r_{12}=r_{23}=r_{13}=r_{14}=r_{24}=r_{34}=1,
$$

e

$$
r_{15}=r_{26}=r_{37}<r_{45}=r_{46}=r_{47}<r_{16}=r_{17}=r_{25}=r_{27} r_{35}=r_{36}<1,
$$

as quais formam uma configuração central. Em verdade, temos uma família a um parâmetro de configurações centrais, de forma que existe uma curva no espaço, que conecta $r_{1}$ ao baricentro 
do tetraedro, e duas cópias desta curva giradas em torno da altura do tetraedro de $2 \pi / 3$ e $4 \pi / 3$, respectivamente, onde podemos colocar $m_{5}, m_{6}$ e $m_{7}$ de modo que as sete massas formem uma configuração central, conforme a figura 5.18.

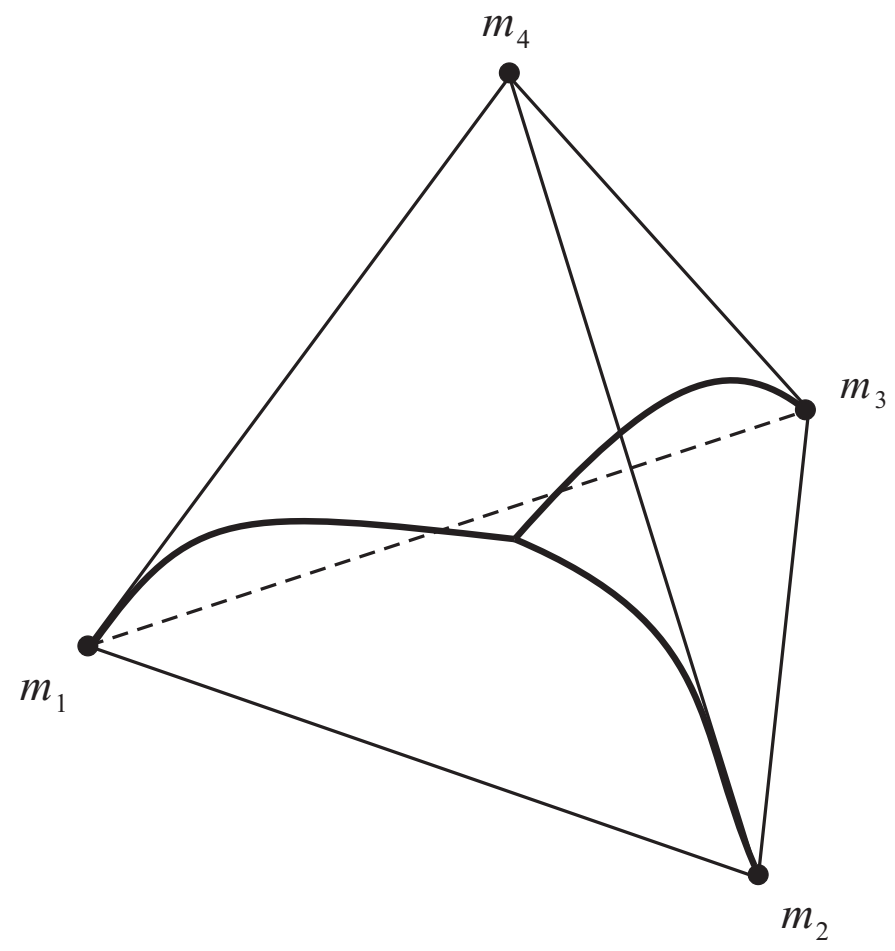

Figura 5.18: Curvas de configurações centrais exemplo de Hampton e Santoprete.

Na linha destas configurações empilhadas com triângulo equilátero temos um caso estudado por Mello e Llibre em [28]. Neste trabalho, eles estudam configurações centrais com cinco massas, nas quais três massas estão sempre nos vértices de um triângulo equilátero e outras duas massas estão sobre a mediatriz do triângulo, vide figura 5.19.

O resultado de Mello e Llibre segue no seguinte teorema, uma prova pode ser vista em [28].

Teorema 5.4.3 Assuma três massas, $m_{1}, m_{2}$ e $m_{3}$, nos vértices de um triângulo equilátero $e$ outras duas massas, $m_{4}$ e $m_{5}$, dispostas sobre a mediatriz que passa por $m_{3}$, veja a figura 5.19 . Sem perda de generalidade, assuma que o comprimento dos lados do triângulo é 1. Duas condições necessárias para termos uma configuração central são: 


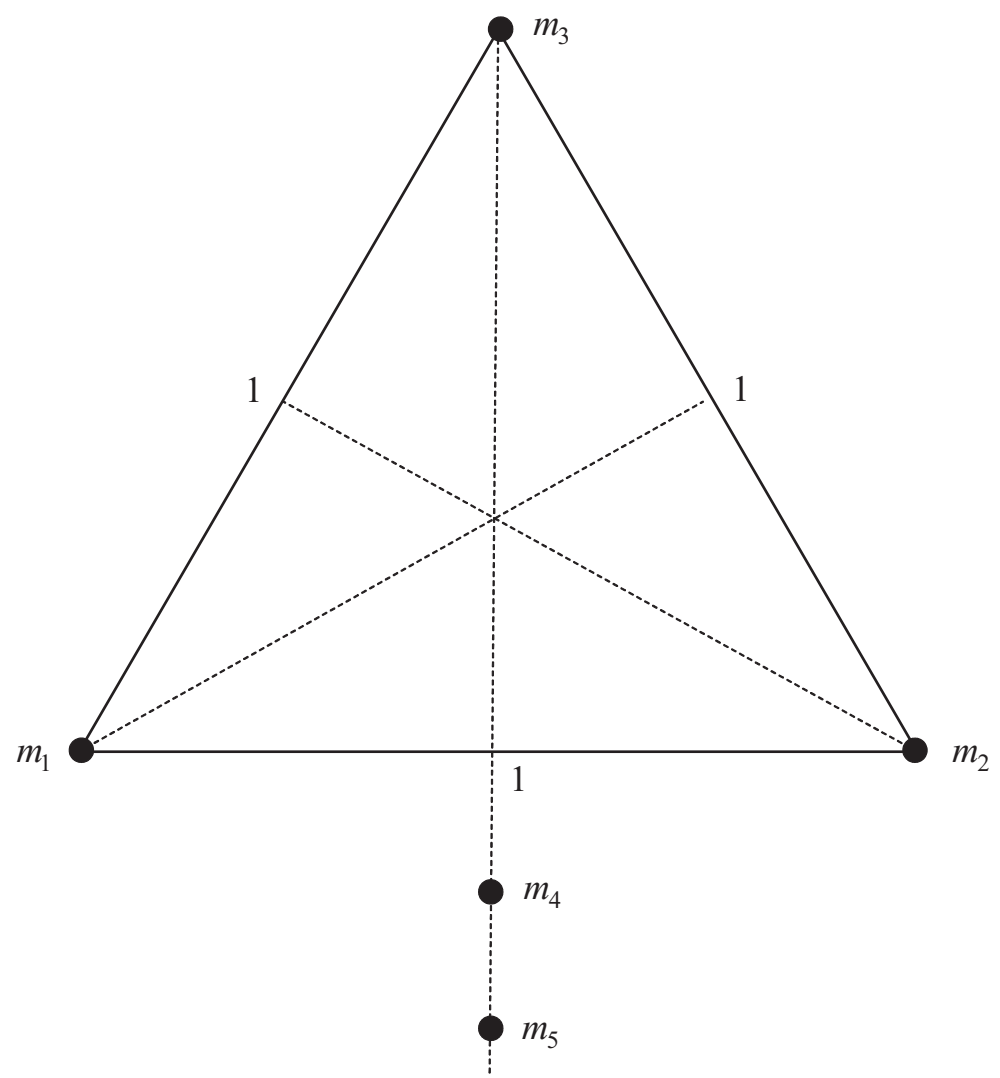

Figura 5.19: Configuração de Mello e Llibre.

a) As duas massas da base, $m_{1}$ e $m_{2}$ devem ser iguais;

b) Uma, e somente uma, das massas deve estar no segmento AB, conforme a figura 5.20.

Sem perda de generalidade, assuma que $m_{4} \in A B$. Então, existe um ponto $G \in A B$, tal que existem segmentos não vazios $I_{1}(G) \subseteq C D, I_{2}(G) \subseteq E A$ e $I_{3}(G) \subseteq B F$ e massas positivas $m_{1}=m_{2}, m_{3}$, $m_{4}$ e $m_{5}(i) \in I_{i}(G), i=1,2,3$, de forma que $m_{1}, m_{2}, m_{3}, m_{4}$ e $m_{5}(i)$ nestas posições formem três classes de configurações centrais, uma para cada i. Veja a figura 5.20.

Assuma que $m_{4} \in A B$, existem evidências numéricas de que para todo ponto $G \in A B$ existam segmentos não vazios $I_{1}(G) \in C D, I_{2}(G) \in E A$ e $I_{3}(G) \in B F$ e massas positivas $m_{1}=m_{2}, m_{3}$, $m_{4}$ e $m_{5}(i) \in I_{i}(G), i=1,2,3$, de forma que $m_{1}, m_{2}, m_{3}, m_{4}$ e $m_{5}(i)$ nestas posições formem três classes de configurações centrais, uma para cada $i$. 


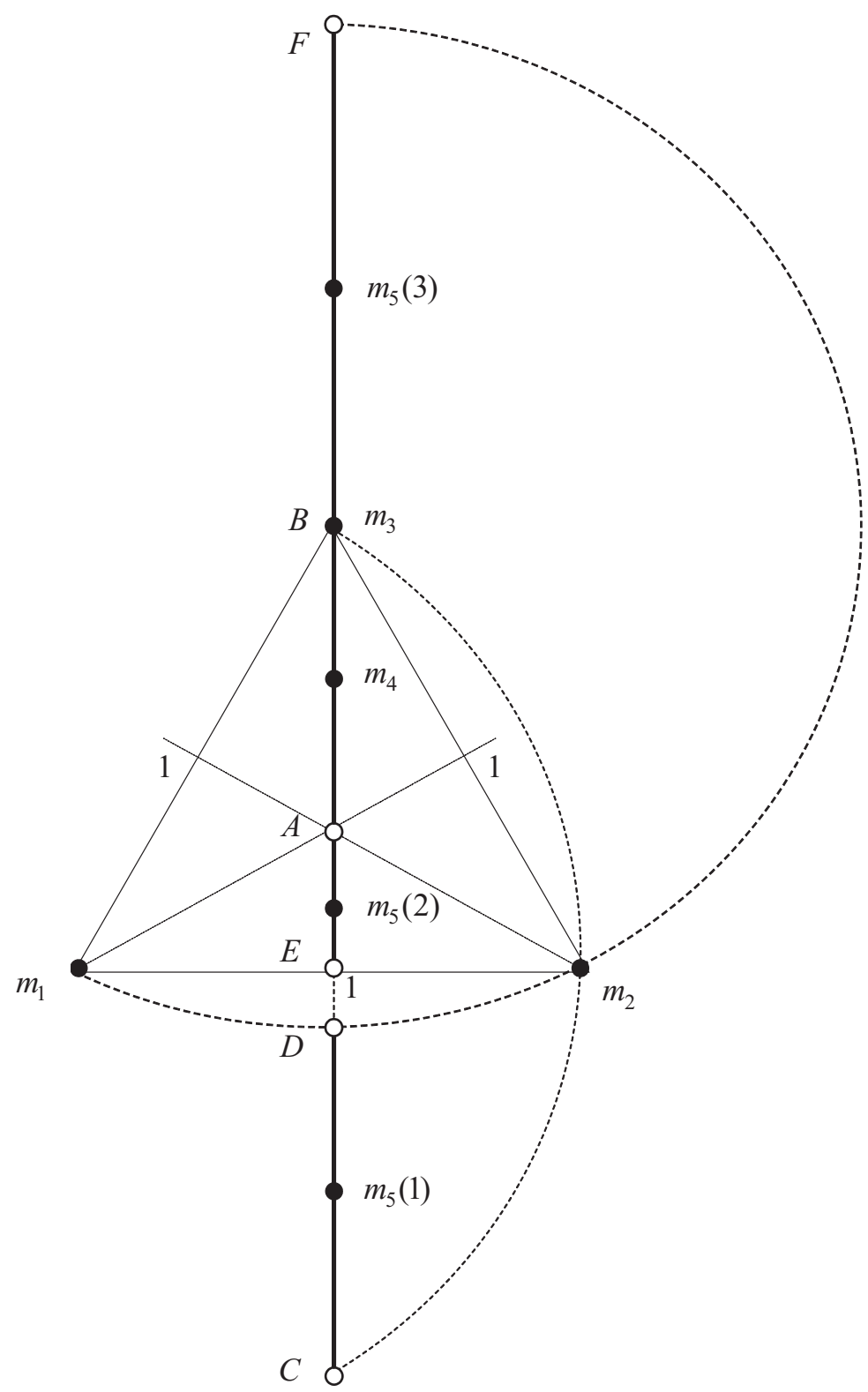

Figura 5.20: Segmentos das configurações centrais de Mello e Llibre.

Novamente, podemos nos perguntar sobre a existência de um análogo espacial para este caso. Ou seja, considerar um problema com quatro massas nos vértices de um tetraedro regular e duas massas colocadas sobre uma reta que passa por um vértice e pelo baricentro da face oposta a este vértice. Este trabalho foi feito por Chaves, Fernandes, Garcia e Mello em [27]. 
Considere um tetraedro regular com lado de comprimento 1 e sejam $A, B, C, D, E$ e $F$ pontos sobre a reta $\mathcal{L}$ que passa pelo vértice em $r_{4}$ e pelo baricentro da face oposta a este vértice. Conforme a figura 5.22 tomemos as seguintes considerações:

1. A sendo o centro geométrico do tetraedro;

2. $B$ é o vértice em $r_{4}$;

3. $C$ é o ponto simétrico ao ponto $B$ relativo ao plano que contém $r_{1}, r_{2}$ e $r_{3}$;

4. E é o baricentro do triângulo equilátero com vértices em $r_{1}, r_{2}$ e $r_{3}$;

5. $D$ e $F$ são os pontos onde a esfera centrada em $B$ e de raio 1 intercepta a reta $\mathcal{L}$;

6. $A B \subset \mathcal{L}$ o segmento com extremidades em $A$ e $B$.

De maneira análoga definimos os segmentos $B F, C D, D E$ e $E A$.

Enunciaremos a seguir o teorema.

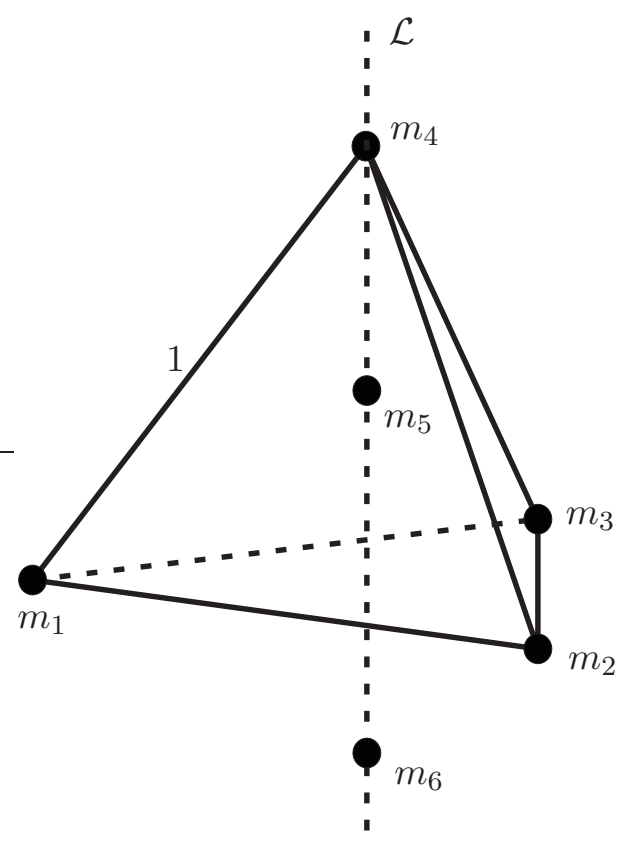

Figura 5.21: Quatro corpos nos vértices de um tetraedro regular e dois sobre a reta $\mathcal{L}$ que conecta um dos vértices e o centro da face oposta. 
Teorema 5.4.4 Assuma que quatro corpos com massas $m_{1}, m_{2}, m_{3}$ e $m_{4}$ estão nos vértices de um tetraedro regular com lados de comprimento 1 e dois corpos de massas $m_{5}$ e $m_{6}$ estão sobre a reta $\mathcal{L}$, de acordo com a figura 5.21. Nestas considerações, os seis corpos formam uma configuração central se as seguintes exigências se verificam:

(a) As três massas $m_{1}, m_{2}$ e $m_{3}$ são iguais;

(b) Somente um dos vetores posições $r_{5}$ ou $r_{6}$ deve estar sobre o segmento AB, veja figura 5.22.

Sem perda de generalidade, assumiremos que $r_{5} \in A B$, sendo que para o caso em que $r_{6} \in A B$ pode-se prosseguir de maneira análoga. Então existem uma posição $G \in A B$, segmentos não vazios $I^{1}(G) \subset C D, I^{2}(G) \subset E A, I^{3}(G) \subset B F$ e massas positivas $m_{1}=m_{2}=m_{3}, m_{4}, m_{5}$ e $m_{6}(i)$, $i=1,2,3$, tais que $r_{1}, r_{2}, r_{3}$ e $r_{4}$ estão nos vértices de um tetraedro regular, $r_{5}$ está em $G$ e $r_{6}(1) \in I^{1}(G)$, ou $r_{6}(2) \in I^{2}(G)$, ou $r_{6}(3) \in I^{3}(G)$, tais que estes corpos formam três configurações centrais. Veja a figura 5.22.

Prova Considere as equações de Andoyer, dadas pelas equações (5.4), para este problema. Como dito anteriormente, o número de equações de Andoyer para o caso espacial de um problema de $n$ corpos é dado por $n(n-1)(n-2) / 2$. Sendo assim, temos para o problema espacial de 6 corpos, um conjunto de 60 equações de Andoyer:

$$
\begin{aligned}
& f_{123}=0, f_{124}=0, f_{125}=0, f_{126}=0, f_{132}=0, f_{134}=0, f_{135}=0, f_{136}=0, f_{142}=0, f_{143}=0, \\
& f_{145}=0, f_{146}=0, f_{152}=0, f_{153}=0, f_{154}=0, f_{156}=0, f_{162}=0, f_{163}=0, f_{164}=0, f_{165}=0, \\
& f_{231}=0, f_{234}=0, f_{235}=0, f_{236}=0, f_{241}=0, f_{243}=0, f_{245}=0, f_{246}=0, f_{251}=0, f_{253}=0, \\
& f_{254}=0, f_{256}=0, f_{261}=0, f_{263}=0, f_{264}=0, f_{265}=0, f_{341}=0, f_{342}=0, f_{345}=0, f_{346}=0, \\
& f_{351}=0, f_{352}=0, f_{354}=0, f_{356}=0, f_{361}=0, f_{362}=0, f_{364}=0, f_{365}=0, f_{451}=0, f_{452}=0, \\
& f_{453}=0, f_{456}=0, f_{461}=0, f_{462}=0, f_{463}=0, f_{465}=0, f_{561}=0, f_{562}=0, f_{563}=0, f_{564}=0 .
\end{aligned}
$$

Afim de darmos um orientação ao espaço, assumiremos nas equações de Andoyer que $\Delta_{1234}>0$. Defina os seguintes subconjuntos de $\mathcal{L}$, veja figura 5.22:

$$
\mathcal{L}_{1}=\left\{r_{i} \in \mathcal{L}: r_{1 i}>1=r_{12}, \Delta_{123 i}<0, i=5,6\right\}
$$




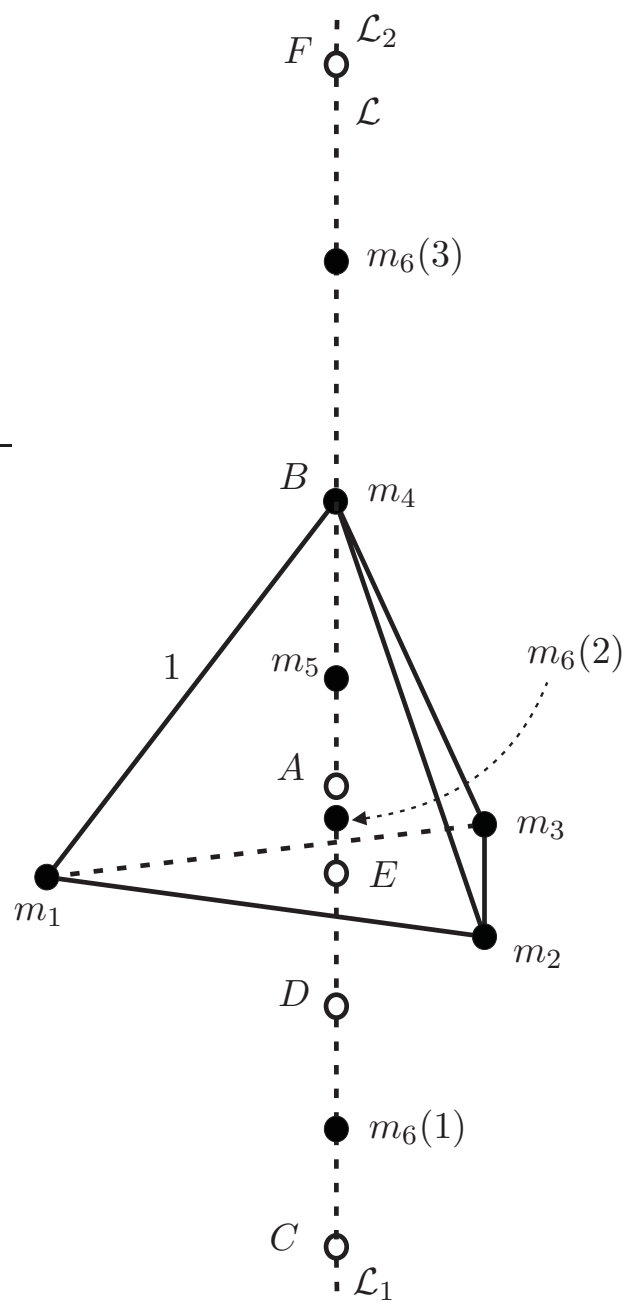

Figura 5.22: Seis corpos em configuração central espacial.

$$
\mathcal{L}_{2}=\left\{r_{i} \in \mathcal{L}: r_{4 i}>1=r_{12}, \Delta_{123 i}>0, i=5,6\right\}
$$

Disto segue

$$
\mathcal{L}=\mathcal{L}_{1} \cup\{C\} \cup C D \cup\{D\} \cup D E \cup\{E\} \cup E A \cup\{A\} \cup A B \cup\{B\} \cup B F \cup\{F\} \cup \mathcal{L}_{2} .
$$

Devido às restrições feitas a priori acerca das configurações a serem estudadas, como na figura 5.21, as seguintes relações, dentre outras, devem ser satisfeitas:

$$
r_{12}=r_{13}=r_{14}=r_{23}=r_{24}=r_{34}=1, \quad r_{15}=r_{25}=r_{35}, \quad r_{16}=r_{26}=r_{36},
$$




$$
\begin{aligned}
& \Delta_{1425}=-\Delta_{1435}=-\Delta_{2415}=\Delta_{2435}=\Delta_{3415}=-\Delta_{3425}, \\
& \Delta_{1426}=-\Delta_{1436}=-\Delta_{2416}=\Delta_{2436}=\Delta_{3416}=-\Delta_{3426}, \\
& \Delta_{1523}=-\Delta_{1532}=-\Delta_{2513}=\Delta_{2531}=\Delta_{3512}=-\Delta_{3521}, \\
& \Delta_{1524}=-\Delta_{1534}=-\Delta_{2514}=\Delta_{2534}=\Delta_{3514}=-\Delta_{3524}, \\
& \Delta_{1526}=-\Delta_{1536}=-\Delta_{2516}=\Delta_{2536}=\Delta_{3516}=-\Delta_{3526}, \\
& \Delta_{2613}=-\Delta_{2631}=-\Delta_{1623}=\Delta_{1632}=\Delta_{3612}=-\Delta_{3621}, \\
& \Delta_{2614}=-\Delta_{2634}=-\Delta_{1624}=\Delta_{1634}=\Delta_{3614}=-\Delta_{3624}, \\
& \Delta_{2615}=-\Delta_{2635}=-\Delta_{1625}=\Delta_{1635}=\Delta_{3615}=-\Delta_{3625},
\end{aligned}
$$

e $\Delta_{456 i}=0$, para $i=1,2,3$ e quaisquer permutações dos índices $i, 4,5,6$, visto que $\Delta_{i j h k}=$ $\left(r_{i}-r_{j}\right) \wedge\left(r_{j}-r_{h}\right) \cdot\left(r_{h}-r_{k}\right)$.

As simetrias especificadas acima, nos dão que as seguintes equações são trivialmente verificadas,

$$
\begin{gathered}
f_{123}=0, f_{124}=0, f_{125}=0, f_{126}=0, f_{132}=0, f_{134}=0, f_{135}=0, f_{136}=0, f_{145}=0, f_{146}=0 \\
f_{231}=0, f_{234}=0, f_{235}=0, f_{236}=0, f_{245}=0, f_{246}=0, f_{345}=0, f_{346}=0, f_{456}=0, f_{465}=0 \mathrm{e} \\
f_{564}=0
\end{gathered}
$$

Isto é, 21 das 60 equações se verificam diretamente. Logo, restam 39 equações a serem verificadas.

Lema 5.4.5 Se a posição do corpo de massa $m_{5}$ é simétrica à posição do corpo de massa $m_{4}$, isto é, $r_{5}$ está em $C$, então não existe posição para a massa $m_{6}$ em $\mathcal{L}$ e massas positivas $m_{i}, i=1, \ldots, 6$, tais que estes corpos formem uma configuração central.

Prova: A configuração imposta pelo lema implica que $r_{12}=r_{15}, r_{16} \neq r_{12}$ e $r_{15} \neq r_{16}$. Disto temos que as equações

$$
f_{142}=0, f_{143}=0, f_{241}=0, f_{243}=0, f_{341}=0 \text { e } f_{342}=0
$$


são equivalentes a uma única equação, dada por

$$
\left(R_{12}-R_{45}\right) \Delta_{1425} m_{5}+\left(R_{16}-R_{46}\right) \Delta_{1426} m_{6}=0 .
$$

Das equações

$$
f_{152}=0, f_{153}=0, f_{251}=0, f_{253}=0, f_{351}=0 \text { e } f_{352}=0,
$$

temos também uma única equação,

$$
\left(R_{12}-R_{45}\right) \Delta_{1524} m_{4}+\left(R_{16}-R_{56}\right) \Delta_{1526} m_{6}=0 .
$$

Note que o coeficiente da massa $m_{5},\left(R_{12}-R_{45}\right) \Delta_{1425}$, na equação (5.30) é positivo. Deste modo, o coeficiente da massa $m_{6},\left(R_{16}-R_{46}\right) \Delta_{1426}$, deve ser negativo. Isto ocorre somente quando $r_{16}>r_{46}$ e $\Delta_{1426}>0$. Isto implica que o coeficiente da massa $m_{6},\left(R_{16}-R_{56}\right) \Delta_{1526}$, na equação (5.31) é negativo. Porém, nas considerações acima temos que o coeficiente da massa $m_{4}$ é negativo. Deste modo, os coeficientes das massas $m_{4}$ e $m_{6}$ devem ter sinais opostos, como $m_{4} \neq 0$ e $m_{6} \neq 0$, ou $m_{4}$ ou $m_{6}$ deve ter valor negativo, o que é uma contradição.

Podemos obter o mesmo resultado se trocarmos a massa $m_{5}$ pela massa $m_{6}$, basta proceder de maneira análoga. O mesmo acontece para os lemas que seguem.

Lema 5.4.6 Se a posição do corpo de massa $m_{5}$ é o ponto A, então não existem posições para o corpo de massa $m_{6}$ em $\mathcal{L}$ e massas positivas $m_{i}, i=1, \ldots, 4$, tais que estes corpos formem uma configuração central.

Prova: Por hipótese, neste caso temos $r_{15}=r_{45}$ e do Lema 5.4.5, temos $r_{16} \neq r_{12}$. Das equações

$$
f_{142}=0, f_{143}=0, f_{241}=0, f_{243}=0, f_{341}=0 \text { e } f_{342}=0,
$$

temos uma única equação

$$
\left(R_{16}-R_{46}\right) \Delta_{1426} m_{6}=0
$$


Como $m_{6}>0$ e $\Delta_{1426} \neq 0$, temos $R_{16}=R_{46}$ ou, equivalentemente, $r_{16}=r_{46}$. Sendo assim $r_{6}=r_{5}$, mas esta é uma configuração de colisão.

Lema 5.4.7 Se a posição da massa $m_{5}$ é o ponto E, então não existe posição para o corpo de massa $m_{6}$ e massas positivas $m_{i}, i=1, \ldots, 6$, tais que estes corpos formem uma configuração central.

Prova:. Por hipótese, $\Delta_{1523}=0$. Das equações

$$
f_{152}=0, f_{153}=0, f_{251}=0, f_{253}=0, f_{351}=0 \text { e } f_{352}=0,
$$

temos uma única equação

$$
\left(R_{12}-R_{45}\right) \Delta_{1524} m_{4}+\left(R_{16}-R_{56}\right) \Delta_{1526} m_{6}=0 .
$$

Das equações

$$
f_{142}=0, f_{143}=0, f_{241}=0, f_{243}=0, f_{341}=0 \text { e } f_{342}=0,
$$

também temos uma única equação

$$
\left(R_{15}-R_{45}\right) \Delta_{1425} m_{5}+\left(R_{16}-R_{46}\right) \Delta_{1426} m_{6}=0 .
$$

Note que o coeficiente da massa $m_{4},\left(R_{12}-R_{45}\right) \Delta_{1524}$, na equação (5.32) é positivo. Deste modo, o coeficiente da massa $m_{6},\left(R_{16}-R_{56}\right) \Delta_{1526}$, deve ser negativo para termos ambas as massas positivas. Isto ocorre somente onde $r_{16}>r_{56}$ e $\Delta_{1526}>0$. Portanto, o coeficiente da massa $m_{5}$, $\left(R_{15}-R_{45}\right) \Delta_{1425}$, na equação (5.33) é positivo. Mas, o coeficiente da massa $m_{6}$ na equação é positivo. Deste modo, as massas $m_{5}$ e $m_{6}$ tem sinais opostos, o que é uma contradição.

Lema 5.4.8 Assuma que temos quatro corpos com massas $m_{1}, m_{2}, m_{3}$ e $m_{4}$ nos vértices de um tetraedro regular com todos os lados de comprimento 1 e dois corpos com massas $m_{5}$ e $m_{6}$ na reta $\mathcal{L}$ 
de acordo com a figura 5.22. Nestas condições, os seis corpos podem estar em configuração central se as seguinte afirmações se verificam:

(a) As três massas $m_{1}, m_{2}$ e $m_{3}$ são iguais;

(b) Somente um dos vetores $r_{5}$ ou $r_{6}$ deve estar no segmento $A B$.

Prova: Dos Lemas 5.4.5, 5.4.6 e 5.4.7 devemos assumir $r_{5} \neq A, r_{5} \neq C, r_{5} \neq E, r_{6} \neq A, r_{6} \neq C$ e $r_{6} \neq E$. Nestas considerações, das equações

$$
f_{254}=0, f_{256}=0, f_{264}=0, f_{265}=0, f_{452}=0, f_{462}=0 \text { e } f_{562}=0
$$

temos uma única equação

$$
\left(R_{12}-R_{16}\right) \Delta_{2641}\left(m_{1}-m_{3}\right)=0
$$

Deste modo, temos $m_{1}=m_{3}$ desde que $r_{12} \neq r_{16}$ e $\Delta_{2641} \neq 0$. Pelo mesmo argumento, das equações

$$
f_{154}=0, f_{156}=0, f_{164}=0, f_{165}=0, f_{451}=0, f_{461}=0 \text { e } f_{561}=0
$$

temos somente uma equação

$$
\left(R_{12}-R_{15}\right) \Delta_{1542}\left(m_{2}-m_{3}\right)=0
$$

Deste modo, temos $m_{2}=m_{3}$ desde que $r_{12} \neq r_{15}$ e $\Delta_{1542} \neq 0$. Das equações

$$
f_{354}=0, f_{356}=0, f_{364}=0, f_{365}=0, f_{453}=0, f_{463}=0 \text { e } f_{563}=0
$$

possuímos somente a equação

$$
\left(R_{12}-R_{15}\right) \Delta_{3541}\left(m_{1}-m_{2}\right)=0
$$

Desde que $r_{12} \neq r_{15}$ e $\Delta_{3541} \neq 0$, temos $m_{1}=m_{2}$. Portanto, $m_{1}=m_{2}=m_{3}$, isto prova o item (a) deste Lema. Assumindo, portanto, $m_{1}=m_{2}=m_{3}$, no Lema 5.4.8, temos mais 21 equações de Andoyer são satisfeitas, portanto, as equações de Andoyer ficam reduzidas a 18 equações, que 
podem ser divididas em 3 grupos de 6 equações, onde em cada grupo as equações são equivalentes a uma única equação, a saber:

(i) As equações

$$
f_{142}=0, f_{143}=0, f_{241}=0, f_{243}=0, f_{341}=0 \text { e } f_{342}=0
$$

são equivalentes à equação

$$
\left(R_{15}-R_{45}\right) \Delta_{1425} m_{5}+\left(R_{16}-R_{46}\right) \Delta_{1426} m_{6}=0 .
$$

(ii) As equações

$$
f_{152}=0, f_{153}=0, f_{251}=0, f_{253}=0, f_{351}=0 \text { e } f_{352}=0,
$$

são equivalentes à equação

$$
\left(R_{12}-R_{15}\right) \Delta_{1523} m_{1}+\left(R_{12}-R_{45}\right) \Delta_{1524} m_{4}+\left(R_{16}-R_{56}\right) \Delta_{1526} m_{6}=0 .
$$

(iii) As equações

$$
f_{162}=0, f_{163}=0, f_{261}=0, f_{263}=0, f_{361}=0 \text { e } f_{362}=0,
$$

são equivalentes à equação

$$
\left(R_{12}-R_{16}\right) \Delta_{1623} m_{1}+\left(R_{12}-R_{46}\right) \Delta_{1624} m_{4}+\left(R_{15}-R_{56}\right) \Delta_{1625} m_{5}=0 .
$$

Os coeficientes da equação (5.34) devem ter sinais opostos. Existem duas possibilidades para isto, ou $\left(R_{15}-R_{45}\right) \Delta_{1425}<0$ e $\left(R_{16}-R_{46}\right) \Delta_{1426}>0$ ou $\left(R_{15}-R_{45}\right) \Delta_{1425}>0$ e $\left(R_{16}-R_{46}\right) \Delta_{1426}<0$. 1) $\left(R_{15}-R_{45}\right) \Delta_{1425}<0$ e $\left(R_{16}-R_{46}\right) \Delta_{1426}>0$. A inequação $\left(R_{15}-R_{45}\right) \Delta_{1425}<0$ é satisfeita somente quando $r_{15}>r_{45}$ e $\Delta_{1425}>0$, ou seja, somente se $r_{5} \in A B$. Já a inequação $\left(R_{16}-\right.$ $\left.R_{46}\right) \Delta_{1426}>0$ é satisfeita quando $r_{16}<r_{46}$, ou seja, $r_{6} \in \mathcal{L}_{1} \cup C D \cup D E \cup E A$, ou $\Delta_{1426}<0$, isto é, $r_{6} \in B F \cup \mathcal{L}_{2}$. 
2) $\left(R_{15}-R_{45}\right) \Delta_{1425}>0$ e $\left(R_{16}-R_{46}\right) \Delta_{1426}<0$. A inequação $\left(R_{15}-R_{45}\right) \Delta_{1425}>0$ é satisfeita quando $r_{15}<r_{45}$, ou seja, $r_{5} \in \mathcal{L}_{1} \cup C D \cup D E \cup E A$, ou $\Delta_{1425}<0$, isto é, $r_{5} \in B F \cup \mathcal{L}_{2}$. Já a inequação $\left(R_{16}-R_{46}\right) \Delta_{1426}<0$ é satisfeita somente se $r_{16}>r_{46}$ e $\Delta_{1426}>0$. Isto ocorre somente quando $r_{6} \in A B$.

Os itens 1) e 2) provam o item (b) do lema, finalizando a demonstração.

Portanto, a primeira parte do Teorema 5.4.4 está demonstrada.

Observação 5.4.9 Como visto no Lema 5.4.8 devemos assumir ou $r_{5}$ ou $r_{6}$ em AB. Sem perda de generalidade, assumiremos que $r_{5} \in A B$. Veja Lema 5.4.8.

Lema 5.4.10 Se $r_{6} \in \mathcal{L}_{1}$, então não existem massas positivas $m_{i}, i=1, \ldots, 6$, tais que estes corpos formem uma configuração central.

Prova: Das hipóteses acima, temos as seguintes relações

$$
\begin{gathered}
R_{12}-R_{16}>0, \quad R_{12}-R_{46}>0, \quad R_{15}-R_{56}>0, \\
\Delta_{1623}<0, \quad \Delta_{1624}<0, \quad \Delta_{1625}<0 .
\end{gathered}
$$

Portanto, os três coeficientes da equação (5.36) são negativos. Isto implica que duas das três massas $m_{1}, m_{4}, m_{5}$ tem sinais opostos. Sendo assim, pelo menos uma massa seria negativa, o que é uma contradição.

Lema 5.4.11 Se $r_{6} \in \mathcal{L}_{2}$, então não existem massas positivas $m_{i}, i=1, \ldots, 6$, tais que estes corpos formem uma configuração central.

Prova: Das hipóteses acima, seguem as relações

$$
R_{12}-R_{16}>0, \quad R_{12}-R_{46}>0, \quad R_{15}-R_{56}>0
$$




$$
\Delta_{1623}>0, \quad \Delta_{1624}>0, \quad \Delta_{1625}>0 .
$$

Portanto, os três coeficientes da equação (5.36) são positivos, implicando que duas das três massas $m_{1}, m_{4}, m_{5}$ possuem sinais opostos, o que é uma contradição.

Lema 5.4.12 Se $r_{6} \in D E$, então não existem massas positivas $m_{i}, i=1, \ldots, 6$, tais que estes corpos formem uma configuração central.

Prova: Das hipóteses acima, temos as relações

$$
\begin{gathered}
R_{12}-R_{16}<0, \quad R_{12}-R_{46}<0, \quad R_{15}-R_{56}<0, \\
\Delta_{1623}<0, \quad \Delta_{1624}<0, \quad \Delta_{1625}<0 .
\end{gathered}
$$

Portanto, os três coeficientes das equações (5.36) são positivos. Isto implica que duas das três massas $m_{1}, m_{4}, m_{5}$ tem sinais opostos, o que é uma contradição.

Sem perda de generalidade, tomaremos um sistema de coordenadas tal que os corpos de massas $m_{1}, m_{2}, m_{3}, m_{4}, m_{5}$ e $m_{6}$ estão nas seguintes posições

$$
(\sqrt{3} / 3,0,0),(-\sqrt{3} / 6,-1 / 2,0),(-\sqrt{3} / 6,1 / 2,0),(0,0, \sqrt{6} / 3),(0,0, x) \text { e }(0,0, y),
$$

respectivamente. Deste modo, temos as seguintes coordenadas para os pontos citados no enunciado.

$$
\begin{gathered}
A=(0,0, \sqrt{6} / 12), B=(0,0, \sqrt{6} / 3), C=(0,0,-\sqrt{6} / 3), D=(0,0,-1+\sqrt{6} / 3), E=(0,0,0) \text { e } \\
F=(0,0,1+\sqrt{6} / 3) .
\end{gathered}
$$

Portanto, $r_{5} \in A B$ se, e somente se, $\sqrt{6} / 12<x<\sqrt{6} / 3$.

Utilizando os coeficientes das equações (5.34), (5.35) e (5.36), respectivamente, definimos as funções

$$
n_{13}=\left(R_{15}-R_{45}\right) \Delta_{1425}, \quad n_{14}=\left(R_{16}-R_{46}\right) \Delta_{1426}
$$




$$
\begin{aligned}
& n_{21}=\left(R_{12}-R_{15}\right) \Delta_{1523}, \quad n_{22}=\left(R_{12}-R_{45}\right) \Delta_{1524}, \quad n_{24}=\left(R_{16}-R_{56}\right) \Delta_{1526}, \\
& n_{31}=\left(R_{12}-R_{16}\right) \Delta_{1623}, \quad n_{32}=\left(R_{12}-R_{46}\right) \Delta_{1624}, \quad n_{33}=\left(R_{15}-R_{56}\right) \Delta_{1625} .
\end{aligned}
$$

Em termos das coordenadas citadas acima, podemos escrever estas equações da seguinte maneira

$$
\begin{aligned}
& n_{13}(x, y)=\left(\frac{\sqrt{2}-\sqrt{3} x}{6}\right)\left(\frac{1}{\left(x^{2}+\frac{1}{3}\right)^{3 / 2}}-\frac{27}{\left((\sqrt{6}-3 x)^{2}\right)^{3 / 2}}\right) \text {, } \\
& n_{14}(x, y)=\left(\frac{\sqrt{2}-\sqrt{3} y}{6}\right)\left(\frac{1}{\left(y^{2}+\frac{1}{3}\right)^{3 / 2}}-\frac{27}{\left((\sqrt{6}-3 y)^{2}\right)^{3 / 2}}\right) \text {, } \\
& n_{21}(x, y)=\frac{\sqrt{3} x}{2}\left(1-\frac{1}{\left(x^{2}+\frac{1}{3}\right)^{3 / 2}}\right) \\
& n_{22}(x, y)=\left(\frac{\sqrt{3} x-\sqrt{2}}{6}\right)\left(1-\frac{27}{\left((\sqrt{6}-3 x)^{2}\right)^{3 / 2}}\right) \\
& n_{24}(x, y)=\left(\frac{x-y}{2 \sqrt{3}}\right)\left(\frac{1}{\left(y^{2}+\frac{1}{3}\right)^{3 / 2}}-\frac{1}{\left((x-y)^{2}\right)^{3 / 2}}\right), \\
& n_{31}(x, y)=-\frac{\sqrt{3} y}{2}\left(1-\frac{1}{\left(y^{2}+\frac{1}{3}\right)^{3 / 2}}\right) \text {, } \\
& n_{32}(x, y)=\left(\frac{\sqrt{2}-\sqrt{3} y}{6}\right)\left(1-\frac{27}{\left((\sqrt{6}-3 y)^{2}\right)^{3 / 2}}\right) \\
& n_{33}(x, y)=\left(\frac{x-y}{2 \sqrt{3}}\right)\left(\frac{1}{\left(x^{2}+\frac{1}{3}\right)^{3 / 2}}-\frac{1}{\left((x-y)^{2}\right)^{3 / 2}}\right) \text {. }
\end{aligned}
$$

As equações (5.34), (5.35), e (5.36) definem três hiperplanos passando pela origem do espaço de parâmetros $\left(m_{1}, m_{4}, m_{5}, m_{6}\right)$. O vetor de massa é paralelo à reta definida pela interseção desses três hiperplanos. Podemos escrever o vetor de massa como $T=\left(T_{1},-T_{2}, T_{3},-T_{4}\right)$, onde 


$$
\begin{aligned}
& T_{1}=n_{13} n_{24} n_{32}+n_{14} n_{22} n_{33}, \\
& T_{2}=n_{13} n_{24} n_{31}+n_{14} n_{21} n_{33}, \\
& T_{3}=n_{14}\left(n_{21} n_{32}-n_{22} n_{31}\right), \\
& T_{4}=n_{13}\left(n_{21} n_{32}-n_{22} n_{31}\right) .
\end{aligned}
$$

Deste modo, teremos massas positivas $m_{1}, m_{4}, m_{5}$ e $m_{6}$ como soluções das equações (5.34), (5.35) e (5.36) se, e somente se, as componentes do vetor de massa $T$ tem o mesmo sinal.

Lema 5.4.13 Consideremos $x=3 / 10$ e $y=-1 / 2$, ou seja $r_{5} \in A B$ e $r_{6} \in C D$. Então existem massas positivas $m_{i}, i=1, \ldots, 6$, tais que estes corpos formem uma configuração central.

Prova: Substituindo $x=3 / 10$ e $y=-1 / 2$ em $n_{13}, n_{14}, n_{21}, n_{22}, n_{24}, n_{31}, n_{32}$ e $n_{33}$ e então, calculando $T_{1}, T_{2}, T_{3}$ e $T_{4}$, segue que

$$
T_{1}\left(\frac{3}{10},-\frac{1}{2}\right)<0, T_{2}\left(\frac{3}{10},-\frac{1}{2}\right)>0, T_{3}\left(\frac{3}{10},-\frac{1}{2}\right)<0, T_{4}\left(\frac{3}{10},-\frac{1}{2}\right)>0 .
$$

Portanto, as componentes do vetor de massa $T$ tem mesmo sinal. Com isso o Lema está provado. Considerando $m_{1}=m_{2}=m_{3}=10$, daremos algumas informações acerca do valor das massas $m_{4}, m_{5}$ e $m_{6}:$

$$
m_{4}=-\frac{90 \theta_{1}}{889 \theta 2},
$$

onde

$$
\begin{array}{r}
\theta_{1}=-169428788777 \sqrt{3} \sqrt{127}+68197707648 \sqrt{3} \sqrt{127} \sqrt{2} \sqrt{7} \\
+96005855232 \sqrt{127} \sqrt{7}-1733111767086 \sqrt{7} \sqrt{2} \\
-1398415595283 \sqrt{3} \sqrt{7}-55810140602 \sqrt{127} \sqrt{2} \\
+7165469970760+277475849024 \sqrt{3} \sqrt{2}
\end{array}
$$




$$
\begin{array}{r}
\theta_{2}=-6885857196 \sqrt{7} \sqrt{2}-3517815545 \sqrt{3} \sqrt{7}+1654729020 \sqrt{127} \sqrt{2} \\
-2168765627 \sqrt{3} \sqrt{127}+12461781528+1743814656 \sqrt{127} \sqrt{7} \\
+159252480 \sqrt{3} \sqrt{127} \sqrt{2} \sqrt{7}+1138062240 \sqrt{3} \sqrt{2}, \\
m_{5}=-\frac{146304 \theta_{3}}{35(3+2 \sqrt{3} \sqrt{2})^{2}(1536 \sqrt{3} \sqrt{127}-16129) \theta_{4}},
\end{array}
$$

onde

$$
\begin{gathered}
\theta_{3}=-9279529102449 \sqrt{3} \sqrt{127}+910848433824 \sqrt{3} \sqrt{127} \sqrt{2} \sqrt{7} \\
+2443137682041 \sqrt{127} \sqrt{7}-3512003097036 \sqrt{7} \sqrt{2} \\
-3064630331483 \sqrt{3} \sqrt{7}-8621348150970 \sqrt{127} \sqrt{2} \\
+21107073194110 \sqrt{3} \sqrt{2}+110325167369661, \\
\theta_{4}=-6885857196 \sqrt{7} \sqrt{2}-3517815545 \sqrt{3} \sqrt{7}+1654729020 \sqrt{127} \sqrt{2} \\
-2168765627 \sqrt{3} \sqrt{127}+12461781528+1743814656 \sqrt{127} \sqrt{7} \\
+159252480 \sqrt{3} \sqrt{127} \sqrt{2} \sqrt{7}+1138062240 \sqrt{3} \sqrt{2}, \\
40320(1881 \sqrt{3} \sqrt{127}-2810 \sqrt{127} \sqrt{2}+16129) \theta_{5} \\
m_{6}=-\frac{1636 \sqrt{3} \sqrt{127}-16129) \theta_{6}}{127(-9+10 \sqrt{3} \sqrt{2})^{2}(27 \sqrt{3} \sqrt{7}+34 \sqrt{7} \sqrt{2}-49)(1536},
\end{gathered}
$$

onde

$$
\begin{array}{r}
\theta_{5}=-9279529102449 \sqrt{3} \sqrt{127}+910848433824 \sqrt{3} \sqrt{127} \sqrt{2} \sqrt{7} \\
+2443137682041 \sqrt{127} \sqrt{7}-3512003097036 \sqrt{7} \sqrt{2} \\
-3064630331483 \sqrt{3} \sqrt{7}-8621348150970 \sqrt{127} \sqrt{2} \\
+21107073194110 \sqrt{3} \sqrt{2}+110325167369661
\end{array}
$$




$$
\begin{array}{r}
\theta_{6}=-6885857196 \sqrt{7} \sqrt{2}-3517815545 \sqrt{3} \sqrt{7}+1654729020 \sqrt{127} \sqrt{2} \\
-2168765627 \sqrt{3} \sqrt{127}+12461781528+1743814656 \sqrt{127} \sqrt{7} \\
+159252480 \sqrt{3} \sqrt{127} \sqrt{2} \sqrt{7}+1138062240 \sqrt{3} \sqrt{2} .
\end{array}
$$

Por um longo, porém elementar, cálculo as equações (5.34), (5.35) e (5.36) são satisfeitas. O extenso cálculo envolvido neste Lema foi corroborado com software Maple 9. Os valores das massas acima, com uma precisão de dez casas decimais, são dados por

$$
m_{4}=6.7458781851, \quad m_{5}=10.1934123989, \quad m_{6}=8.0305718049 .
$$

Lema 5.4.14 Consideremos $x=3 / 10$ e $y=1 / 10$, ou seja $r_{5} \in A B$ e $r_{6} \in E A$. Então existem massas positivas $m_{i}, i=1, \ldots, 6$, tais que estes corpos formem uma configuração central.

Prova: Substituindo $x=3 / 10$ e $y=1 / 10$ em $n_{13}, n_{14}, n_{21}, n_{22}, n_{24}, n_{31}, n_{32}$ e $n_{33}$ e calculando $T_{1}, T_{2}, T_{3}$ e $T_{4}$, segue que

$$
T_{1}\left(\frac{3}{10}, \frac{1}{10}\right)>0, T_{2}\left(\frac{3}{10}, \frac{1}{10}\right)<0, T_{3}\left(\frac{3}{10}, \frac{1}{10}\right)>0, T_{4}\left(\frac{3}{10}, \frac{1}{10}\right)<0
$$

Portanto, as componentes do vetor de massa $T$ tem mesmo sinal. Com isso o lema está provado.

Considerando as massas $m_{1}=m_{2}=m_{3}=10$, temos que as massas $m_{4}, m_{5}$ e $m_{6}$ assumem o seguintes valores:

$$
m_{4}=\frac{90 \psi_{1}}{13081 \psi_{2}}
$$


onde

$$
\begin{array}{r}
\psi_{1}=400775035943835 \sqrt{3} \sqrt{103}-367935503618123 \sqrt{3} \sqrt{127} \\
-79367052441600 \sqrt{127} \sqrt{103}+340184683326250 \sqrt{127} \sqrt{2} \\
-446181650457150 \sqrt{2} \sqrt{103}-391767956870560 \sqrt{3} \sqrt{2} \\
+42152670665280 \sqrt{3} \sqrt{127} \sqrt{2} \sqrt{103}+1140836863198798,
\end{array}
$$

$$
\begin{array}{r}
\psi_{2}=-828144000 \sqrt{127} \sqrt{103}+155520000 \sqrt{3} \sqrt{127} \sqrt{2} \sqrt{103} \\
-336046827905 \sqrt{3} \sqrt{103}+123201043920 \sqrt{3} \sqrt{2} \\
+344038847249 \sqrt{3} \sqrt{127}+578025295560 \sqrt{2} \sqrt{103} \\
-449497813320 \sqrt{127} \sqrt{2}-656045558874
\end{array}
$$

$$
m_{5}=\frac{4572 \psi_{3}}{515(24 \sqrt{3} \sqrt{127}-16129)(-3+10 \sqrt{3} \sqrt{2})^{2} \psi_{4}},
$$

onde

$$
\begin{gathered}
\psi_{3}=277476547044902110 \sqrt{3} \sqrt{103}-208952726731151670 \sqrt{3} \sqrt{127} \\
+223267858814565828 \sqrt{127} \sqrt{2}-298005698143418421 \sqrt{2} \sqrt{103} \\
-2925421091076103921 \sqrt{3} \sqrt{2}-118173007491268230 \sqrt{127} \sqrt{103} \\
+51801039529144776 \sqrt{3} \sqrt{127} \sqrt{2} \sqrt{103}+5985425952439496070 \\
\psi_{4}=-828144000 \sqrt{127} \sqrt{103}+155520000 \sqrt{3} \sqrt{127} \sqrt{2} \sqrt{103} \\
-336046827905 \sqrt{3} \sqrt{103}+123201043920 \sqrt{3} \sqrt{2} \\
+344038847249 \sqrt{3} \sqrt{127}-656045558874 \\
+578025295560 \sqrt{2} \sqrt{103}-449497813320 \sqrt{127} \sqrt{2},
\end{gathered}
$$




$$
m_{6}=-\frac{3708(-1881 \sqrt{3} \sqrt{127}+2810 \sqrt{127} \sqrt{2}-16129) \psi_{5}}{635(24 \sqrt{3} \sqrt{127}-16129)(-603 \sqrt{3} \sqrt{103}+2090 \sqrt{2} \sqrt{103}-10609) \psi_{6}}
$$

onde

$$
\begin{array}{r}
\psi_{5}=277476547044902110 \sqrt{3} \sqrt{103}-208952726731151670 \sqrt{3} \sqrt{127} \\
+223267858814565828 \sqrt{127} \sqrt{2}-298005698143418421 \sqrt{2} \sqrt{103} \\
-2925421091076103921 \sqrt{3} \sqrt{2}-118173007491268230 \sqrt{127} \sqrt{103} \\
+51801039529144776 \sqrt{3} \sqrt{127} \sqrt{2} \sqrt{103}+5985425952439496070 \\
\psi_{6}=(-9+10 \sqrt{3} \sqrt{2})^{2}(-828144000 \sqrt{127} \sqrt{103}-656045558874 \\
+155520000 \sqrt{3} \sqrt{127} \sqrt{2} \sqrt{103}-336046827905 \sqrt{3} \sqrt{103} \\
+123201043920 \sqrt{3} \sqrt{2}+344038847249 \sqrt{3} \sqrt{127} \\
+578025295560 \sqrt{2} \sqrt{103}-449497813320 \sqrt{127} \sqrt{2}) .
\end{array}
$$

Por um longo, porém elementar, cálculo as equações (5.34), (5.35) e (5.36) são satisfeitas. O extenso cálculo envolvido neste lema foi corroborado com o software Maple 9. Os valores das massas acima, com uma precisão de dez casas decimais, são

$$
m_{4}=8.0398208730, \quad m_{5}=0.0828851657, \quad m_{6}=0.0962271225 .
$$

Lema 5.4.15 Consideremos $x=3 / 10$ e $y=1$, ou seja $r_{5} \in A B$ e $r_{6} \in B F$. Então existem massas positivas $m_{i}, i=1, \ldots, 6$, tais que estes corpos formem uma configuração central.

Prova: Substituindo $x=3 / 10$ e $y=1$ em $n_{13}, n_{14}, n_{21}, n_{22}, n_{24}, n_{31}, n_{32}$ e $n_{33}$ e calculando $T_{1}$, $T_{2}, T_{3}$ e $T_{4}$, segue que

$$
T_{1}\left(\frac{3}{10}, 1\right)>0, \quad T_{2}\left(\frac{3}{10}, 1\right)<0, \quad T_{3}\left(\frac{3}{10}, 1\right)>0, \quad T_{4}\left(\frac{3}{10}, 1\right)<0 .
$$


Portanto, as componentes do vetor de massa $T$ tem mesmo sinal. Assim o lema está provado.

Considerando as massas $m_{1}=m_{2}=m_{3}=10$, temos as seguintes informações acerca das massas $m_{4}, m_{5}$ e $m_{6}:$

$$
m_{4}=\frac{45 \chi_{1}}{508 \chi_{2}}
$$

onde

$$
\begin{array}{r}
\chi_{1}=53153630040 \sqrt{3} \sqrt{127} \sqrt{2}-218046103912 \sqrt{127} \sqrt{2}+1243241083487 \\
+169782922943 \sqrt{3} \sqrt{127}-129631817952 \sqrt{127}-1242920324976 \sqrt{3} \\
-430417266086 \sqrt{3} \sqrt{2}+1513598400600 \sqrt{2}, \\
\chi_{2}=-302306823 \sqrt{127}+127894410 \sqrt{3} \sqrt{127} \sqrt{2}+831828240 \sqrt{127} \sqrt{2} \\
-644882824 \sqrt{3} \sqrt{127}-4369346100 \sqrt{2}+3421428641 \sqrt{3} \\
-4211991576+1781931920 \sqrt{3} \sqrt{2}, \\
56007 \chi_{3} \\
m_{5}=-\frac{1029 \sqrt{3} \sqrt{127}-16129)(-3+\sqrt{3} \sqrt{2})^{2} \chi_{4}}{80(1029}
\end{array}
$$

onde

$$
\begin{array}{r}
\chi_{3}=587871472464 \sqrt{3} \sqrt{127} \sqrt{2}-2792763123759 \sqrt{127} \sqrt{2}-31866467162793 \\
+2357179926093 \sqrt{3} \sqrt{127}-1447895560896 \sqrt{127}-4363013925568 \sqrt{3} \\
+12332808521953 \sqrt{3} \sqrt{2}+5395481085936 \sqrt{2}, \\
\chi_{4}=-302306823 \sqrt{127}+127894410 \sqrt{3} \sqrt{127} \sqrt{2}+831828240 \sqrt{127} \sqrt{2} \\
-644882824 \sqrt{3} \sqrt{127}-4369346100 \sqrt{2}+3421428641 \sqrt{3} \\
-4211991576+1781931920 \sqrt{3} \sqrt{2},
\end{array}
$$




$$
m_{6}=\frac{4410(-1881 \sqrt{3} \sqrt{127}+2810 \sqrt{127} \sqrt{2}-16129) \chi_{5}}{127(1029 \sqrt{3} \sqrt{127}-16129)(-9 \sqrt{3}+11 \sqrt{2}+8)(-9+10 \sqrt{3} \sqrt{2})^{2} \chi_{6}}
$$

onde

$$
\begin{array}{r}
\chi_{5}=587871472464 \sqrt{3} \sqrt{127} \sqrt{2}-2792763123759 \sqrt{127} \sqrt{2}-31866467162793 \\
+2357179926093 \sqrt{3} \sqrt{127}-1447895560896 \sqrt{127}-4363013925568 \sqrt{3} \\
+12332808521953 \sqrt{3} \sqrt{2}+5395481085936 \sqrt{2} \\
\chi_{6}=-302306823 \sqrt{127}+127894410 \sqrt{3} \sqrt{127} \sqrt{2}+831828240 \sqrt{127} \sqrt{2} \\
-644882824 \sqrt{3} \sqrt{127}-4369346100 \sqrt{2}+3421428641 \sqrt{3} \\
-4211991576+1781931920 \sqrt{3} \sqrt{2} .
\end{array}
$$

Por um longo, porém elementar cálculo, temos que as equações (5.34), (5.35) e (5.36) são satisfeitas. O extenso cálculo envolvido no lema foi corroborado com o software Maple 9. Os valores das massas acima, com uma precisão de dez casas decimais, são

$$
m_{4}=2.8165087541, \quad m_{5}=145.0492841643, \quad m_{6}=9.1870655797 .
$$

Defina

$$
\begin{gathered}
G=(0,0,3 / 10) \in A B, G_{1}=(0,0,-1 / 2) \in I^{1}(G) \subset C D, G_{2}=(0,0,1 / 10) \in I^{2}(G) \subset E A, \mathrm{e} \\
G_{3}=(0,0,1) \in I^{3}(G) \subset B F .
\end{gathered}
$$

A prova da última parte do Teorema 5.4.4 segue dos Lemas 5.4.13, 5.4.14 e 5.4.15 e a condição aberta dos vetores de massa $T$. Veja figuras 5.23, 5.24 e 5.25.

Consideremos $r_{5} \in A B$ e $r_{6} \in C D$. Segue que

$$
n_{13}<0, n_{14}>0, n_{21}<0, n_{22}>0, n_{24}>0, n_{31}>0, n_{32}<0 .
$$




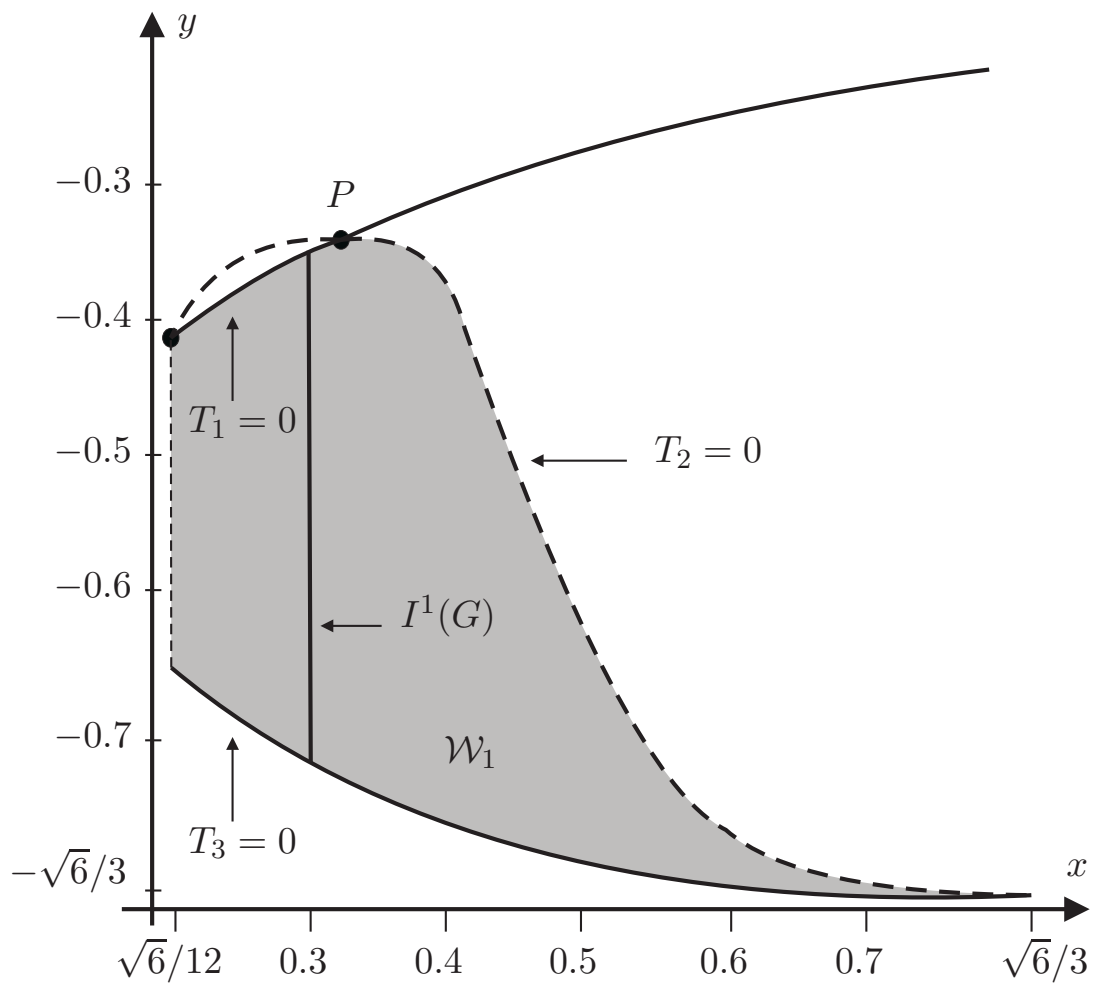

Figura 5.23: A região $\mathcal{W}_{1}$ e o segmento $I^{1}(G)$ do Teorema 5.4.4.

Em particular, como $n_{13} \neq 0$ e $n_{14} \neq 0$, segue que $T_{3}=0$ se, e somente se, $T_{4}=0$. Defina a região

$$
\mathcal{W}_{1}=\left\{(x, y): \frac{\sqrt{6}}{12}<x<\frac{\sqrt{6}}{3},-\frac{\sqrt{6}}{3}<y<\frac{\sqrt{6}}{3}-1, T_{1}<0, T_{2}>0, T_{3}<0, T_{4}>0\right\}
$$

Na figura 5.23 a região $\mathcal{W}_{1}$ é destacada. Note que $(3 / 10,-1 / 2) \in I^{1}(G) \subset \mathcal{W}_{1}$ de acordo com o Teorema 5.4.4. Note também que a projeção da região $\mathcal{W}_{1}$ no eixo dos $x$ é todo segmento $A B$. $\mathrm{O}$ ponto $P$ é definido pela interseção das curvas $T_{1}=0$ e $T_{2}=0$ e temos as coordenadas $x=1 / 3$ e $y=-1 / 3$.

Consideremos $r_{5} \in A B$ e $r_{6} \in E A$. Segue que

$$
n_{13}<0, n_{14}>0, n_{21}<0, n_{22}>0, n_{31}<0, n_{32}>0 \text {. }
$$

Em particular, como $n_{13} \neq 0$ e $n_{14} \neq 0$, segue que $T_{3}=0$ se, e somente se, $T_{4}=0$. Além disso a 


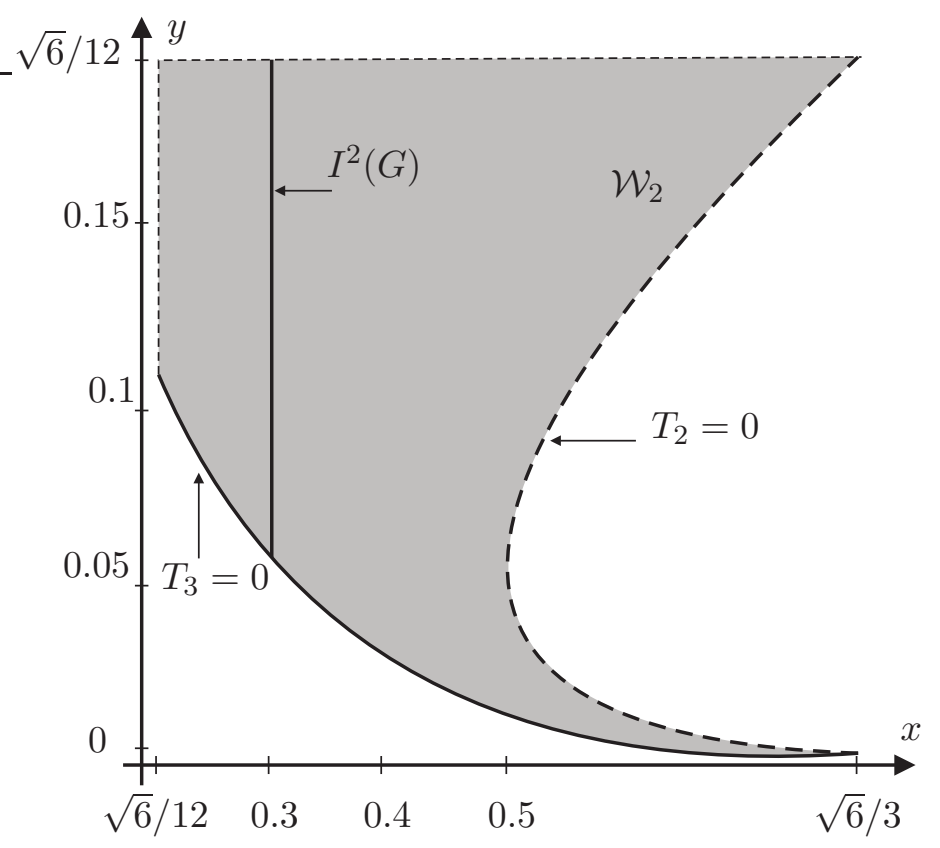

Figura 5.24: A região $\mathcal{W}_{2}$ e o segmento $I^{2}(G)$ do Teorema 5.4.4.

função $T_{1}$ é positiva. Defina a região

$$
\mathcal{W}_{2}=\left\{(x, y): \frac{\sqrt{6}}{12}<x<\frac{\sqrt{6}}{3}, 0<y<\frac{\sqrt{6}}{12}, T_{1}>0, T_{2}<0, T_{3}>0, T_{4}<0\right\} .
$$

A região $\mathcal{W}_{2}$ é destacada na figura 5.24. Note que $(3 / 10,1 / 10) \in I^{2}(G) \subset \mathcal{W}_{2}$ de acordo com o Teorema 5.4.4. Note também que a projeção da região $\mathcal{W}_{2}$ no eixo $x$ é todo segmento $A B$.

Consideremos $r_{5} \in A B$ e $r_{6} \in B F$. Segue que

$$
n_{13}<0, n_{14}>0, n_{21}<0, n_{22}>0, n_{24}>0, n_{31}>0, n_{32}<0 .
$$

Em particular, como $n_{13} \neq 0$ e $n_{14} \neq 0$, segue que $T_{3}=0$ se, e somente se, $T_{4}=0$. Além disso a função $T_{1}$ é positiva. Defina a região

$$
\mathcal{W}_{3}=\left\{(x, y): \frac{\sqrt{6}}{12}<x<\frac{\sqrt{6}}{3}, \frac{\sqrt{6}}{3}<y<1+\frac{\sqrt{6}}{3}, T_{1}>0, T_{2}<0, T_{3}>0, T_{4}<0\right\}
$$

Na figura 5.25 a região $\mathcal{W}_{3}$ está destacada. Note que $(3 / 10,1) \in I^{3}(G) \subset \mathcal{W}_{3}$ de acordo com o Teorema 5.4.4. Note também que a projeção da região $\mathcal{W}_{3}$ no eixo dos $x$ é todo segmento $A B$. 


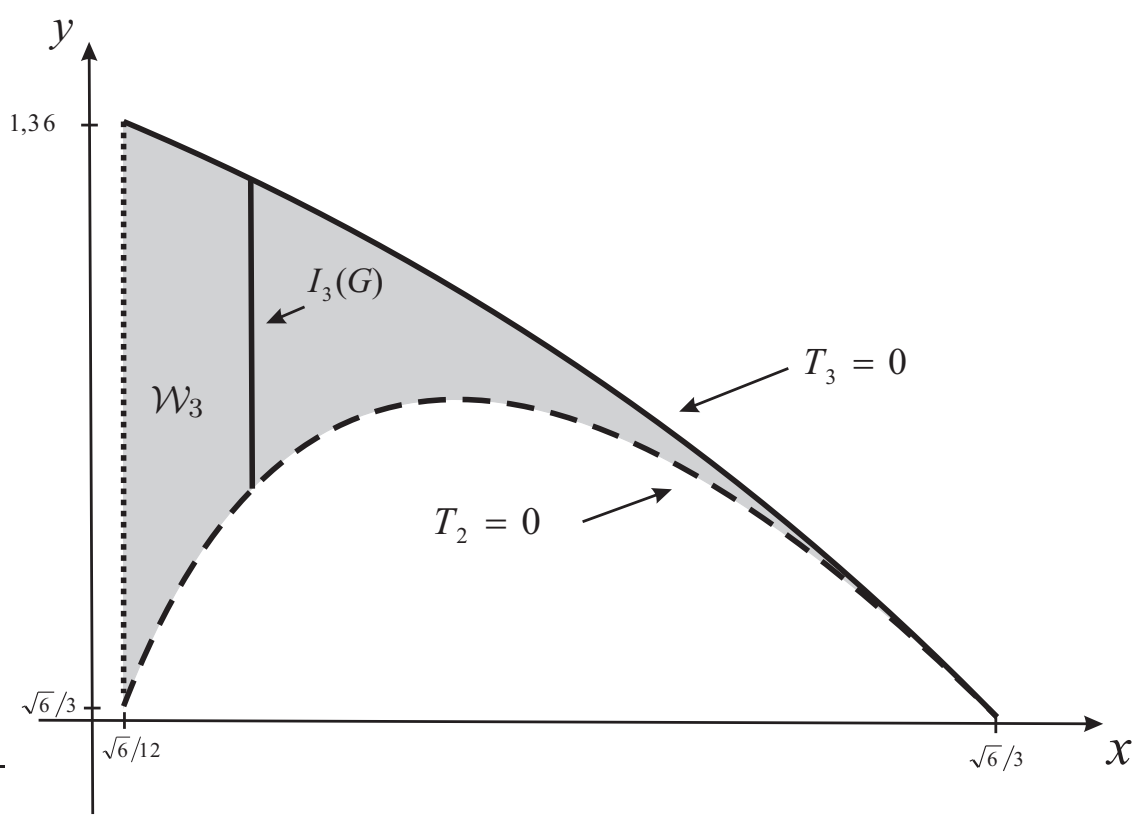

Figura 5.25: A região $\mathcal{W}_{3}$ e o segmento $I^{3}(G)$ do Teorema 5.4.4.

Seguindo a linha da configuração empilhada de Hampton, podemos nos perguntar se seria possível colocar duas massas simetricamente em relação a uma mediatriz do triângulo equilátero, porém no exterior do triângulo. Este caso foi estudado por Mello, Llibre e Perez-Chavela em [29]. Neste trabalho eles estudaram configurações centrais com cinco massas, nas quais três massas estão sempre nos vértices de um triângulo equilátero e outras duas massas estão colocadas simetricamente em relação à mediatriz do triângulo, mas fora do triângulo vide figura 5.26.

O resultado obtido por Mello, Llibre e Perez-Chavela é dado no teorema seguinte. A prova pode ser encontrada em [29].

Teorema 5.4.16 Assuma três massas, $m_{1}, m_{2}$ e $m_{3}$, nos vértices de um triângulo equilátero e duas massas, $m_{4}$ e $m_{5}$, colocadas simetricamente em relação a mediatriz que passas por $r_{3}$. Então existe uma curva simétrica com relação a mediatriz, tal que sobre esta curva há um ponto $P_{2}$ (e seu simétrico $P_{2}^{\prime}$ ), para o qual existem massas positivas de modo que a configuração seja central. Por continuidade, pontos próximo a $P_{2}$ também terão esta propriedade, vide figura 5.2\%.

Embora este resultado seja parecido com o de Hampton, sua análise é muito mais complexa. Por 


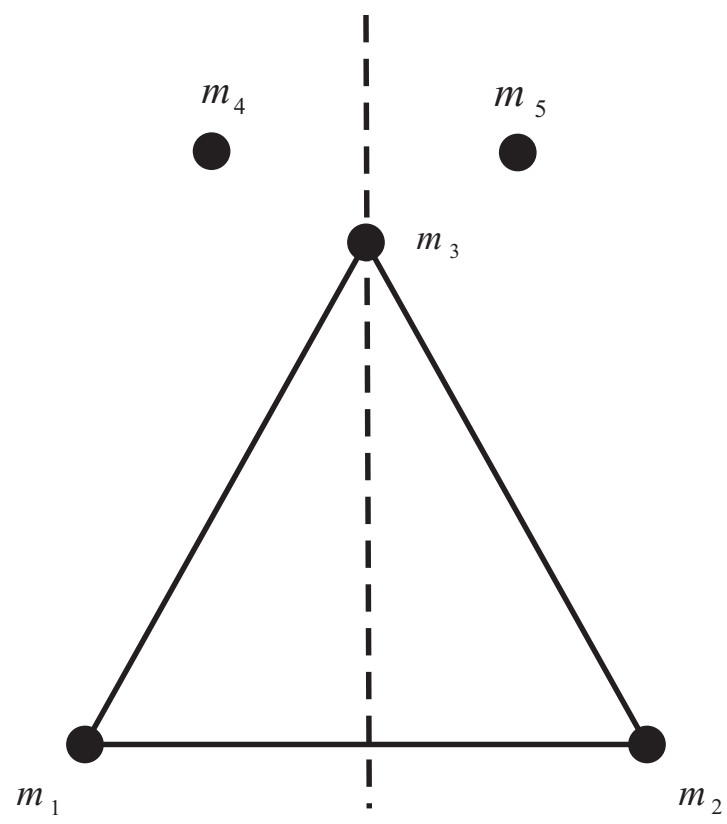

Figura 5.26: Configuração de Mello, Llibre e Perez-Chavela .

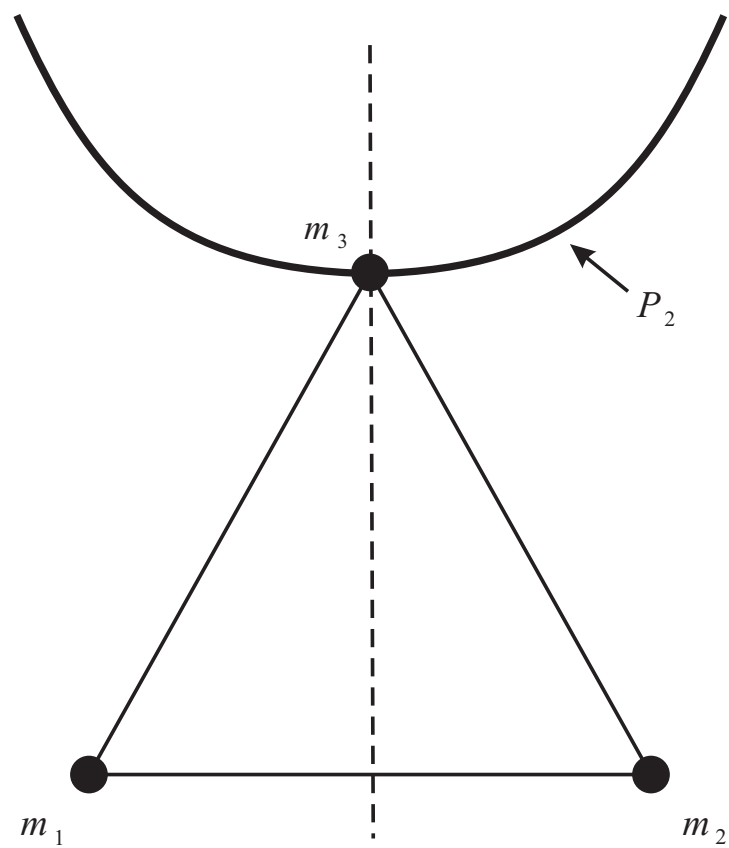

Figura 5.27: Curva configurações centrais de Mello, Llibre e Perez-Chavela .

exemplo, no artigo [29] é mostrado que não é possível escolher $P_{2}$ próximo do vértice que contém $r_{3}$ 
Vendo todos estes casos onde uma configuração espacial é de certa forma generalização de uma planar, podemos nos perguntar se isto sempre é possível para este caso estudado por Mello, Llibre e Perez-Chavela? A resposta é sim e vem dada a seguir.

Com o intuito de completar o resultado de Hampton e Santoprete em [14], vamos considerar aqui o seguinte problema: estudar a possibilidade de existência de uma configuração central com quatro corpos nos vértices de um tetraedro regular e três corpos nos vértices de um triângulo equilátero contido num plano paralelo a alguma das faces, mas no exterior do tetraedro. Mais precisamente, vamos considerar configurações com sete corpos satisfazendo: os vetores posição $r_{1}, r_{2}, r_{3}$ e $r_{4}$ nos vértices de um tetraedro regular $\mathcal{T}_{1}$; os vetores posição $r_{5}, r_{6}$ e $r_{7}$ nos vértices de um triângulo equilátero $\mathcal{E}_{1}$ contido num plano paralelo ao plano que contém $r_{1}, r_{2}$ e $r_{3}$, mas no exterior de $\mathcal{T}_{1}$, vide figura 5.28.

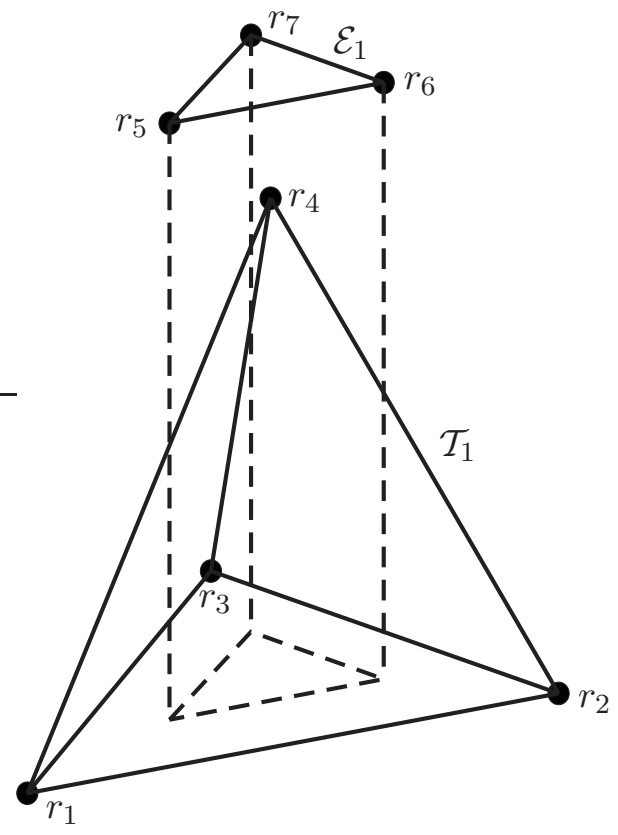

Figura 5.28: Vetores posição $r_{1}, r_{2}, r_{3}$ e $r_{4}$ nos vértices de $\mathcal{T}_{1}$ e vetores posição $r_{5}, r_{6}$ e $r_{7}$ nos vértices de $\mathcal{E}_{1}$.

O resultado que temos é o seguinte.

Teorema 5.4.17 Considere uma configuração espacial como na figura 5.28. Existem vetores posição $r_{1}, r_{2}, r_{3}$ e $r_{4}$ nos vértices de um tetraedro regular $\mathcal{T}_{1}$, vetores posição $r_{5}, r_{6}$ e $r_{7}$ nos vértices de um triângulo equilátero $\mathcal{E}_{1}$ no exterior de $\mathcal{T}_{1}$ e massas positivas $m_{1}=m_{2}=m_{3}, m_{4}$, 
$m_{5}=m_{6}=m_{7}$ tais que com estas posições e massas os sete corpos formam uma configuração central empilhada.

Prova: Vamos usar as equações de Andoyer com volumes, $f_{i j l}=0$. Com os índices pertinentes temos 105 equações para analisar. Porém, devido às simetrias impostas no Teorema, temos

$$
\begin{aligned}
& R_{12}=R_{13}=R_{14}=R_{23}=R_{24}=R_{34}, \\
& R_{15}=R_{26}=R_{37}, \\
& R_{45}=R_{46}=R_{47}, \\
& R_{56}=R_{57}=R_{67}, \\
& R_{16}=R_{17}=R_{25}=R_{27}=R_{35}=R_{36} .
\end{aligned}
$$

As equações (5.37) implicam em várias simetrias também nos volumes com sinais, por exemplo

$$
\begin{aligned}
& \Delta_{1235}=\Delta_{1236}=\Delta_{1237}, \\
& \Delta_{1245}=\Delta_{1246}=\Delta_{1345}=\Delta_{1347}=\Delta_{2346}=\Delta_{2347},
\end{aligned}
$$

entre outras. Usando estas simetrias temos o seguinte resultado.

Lema 5.4.18 Uma condição necessária para termos uma configuração central de acordo com a figura 5.28 é que as massas $m_{1}, m_{2}$ e $m_{3}$ sejam iguais.

Prova: É suficiente considerar as equações $f_{564}=0$ e $f_{574}=0$. Usando as simetrias temos

$$
\begin{aligned}
& f_{564}=\left(m_{1}-m_{2}\right)\left(R_{15}-R_{16}\right) \Delta_{5641}=0, \\
& f_{574}=\left(m_{1}-m_{3}\right)\left(R_{15}-R_{17}\right) \Delta_{5741}=0 .
\end{aligned}
$$

Para a classe de configurações considerada temos $R_{15} \neq R_{16}, R_{15} \neq R_{17}, \Delta_{5641} \neq 0$ e $\Delta_{5741} \neq 0$. Então, as equações acima são satisfeitas se, e somente se, $m_{1}=m_{2}=m_{3}$.

Lema 5.4.19 Uma condição necessária para termos uma configuração central de acordo com a figura 5.28 é que as massas $m_{5}, m_{6}$ e $m_{7}$ sejam iguais. 
Prova: É suficiente considerar as equações $f_{124}=0$ e $f_{134}=0$. Usando as simetrias temos

$$
\begin{aligned}
& f_{124}=\left(m_{5}-m_{6}\right)\left(R_{15}-R_{25}\right) \Delta_{1245}=0, \\
& f_{134}=\left(m_{5}-m_{7}\right)\left(R_{15}-R_{35}\right) \Delta_{1345}=0 .
\end{aligned}
$$

Para a classe de configurações considerada temos $R_{15} \neq R_{25}, R_{15} \neq R_{35}, \Delta_{1245} \neq 0$ e $\Delta_{1345} \neq 0$. Então, as equações acima são satisfeitas se, e somente se, $m_{5}=m_{6}=m_{7}$.

Devido aos Lemas 5.4.18 e 5.4.19, daqui por diante nesta prova nós restringiremos o conjunto de massas admissíveis a $M=m_{1}=m_{2}=m_{3}, m_{4}$ e $m=m_{5}=m_{6}=m_{7}$.

Usando agora, além das simetrias, as condições sobre as massas temos que as seguintes equações são trivialmente satisfeitas.

$$
\begin{aligned}
& f_{123}=f_{124}=f_{125}=f_{126}=f_{127}=0, \\
& f_{132}=f_{134}=f_{135}=f_{136}=f_{137}=0, \\
& f_{154}=f_{264}=f_{347}=f_{374}=f_{451}=0, \\
& f_{231}=f_{234}=f_{235}=f_{236}=f_{237}=0, \\
& f_{462}=f_{473}=f_{561}=f_{562}=f_{563}=0, \\
& f_{564}=f_{567}=f_{571}=f_{572}=f_{573}=0, \\
& f_{574}=f_{576}=f_{671}=f_{672}=f_{673}=0, \\
& f_{674}=f_{675}=0 .
\end{aligned}
$$

Das nossas 105 equações iniciais temos agora apenas 68 equações restantes. Contudo, usando as simetrias e condições sobre as massas é simples ver que temos apenas quatro conjuntos indepen- 
dentes, os quais seguem

$$
f_{147}=0, \quad f_{162}=0, \quad f_{163}=0, \quad f_{164}=0 .
$$

Estas quatro equações podem ser escritas como

$$
\left(\begin{array}{cccc}
h_{11} & 0 & 0 & 0 \\
0 & h_{22} & h_{23} & h_{24} \\
0 & h_{32} & h_{33} & h_{34} \\
0 & h_{42} & 0 & h_{44}
\end{array}\right)\left(\begin{array}{c}
m \\
M \\
m_{4} \\
m
\end{array}\right)=\left(\begin{array}{l}
0 \\
0 \\
0 \\
0
\end{array}\right)
$$

onde

$$
\begin{gathered}
h_{11}=\left(R_{15}-R_{45}\right) \Delta_{1475}+\left(R_{16}-R_{46}\right) \Delta_{1476}, \\
h_{22}=\left(R_{13}-R_{36}\right) \Delta_{1623}, \\
h_{23}=\left(R_{14}-R_{46}\right) \Delta_{1624}, \\
h_{24}=\left(R_{17}-R_{67}\right) \Delta_{1627}, \\
h_{32}=\left(R_{12}-R_{26}\right) \Delta_{1632}, \\
h_{33}=\left(R_{14}-R_{46}\right) \Delta_{1634}, \\
h_{34}=\left(R_{15}+R_{17}-2 R_{56}\right) \Delta_{1635}, \\
h_{42}=\left(R_{12}-R_{26}\right) \Delta_{1642}+\left(R_{13}-R_{36}\right) \Delta_{1643}, \\
h_{44}=\left(R_{15}-R_{56}\right) \Delta_{1645}+\left(R_{17}-R_{67}\right) \Delta_{1647} .
\end{gathered}
$$

Assim, para termos configurações centrais de acordo com a figura 5.28 precisamos encontrar soluções positivas $M, m_{4}$ e $m$ de

$$
H X=0
$$


onde

$$
H=\left(\begin{array}{ccc}
h_{22} & h_{23} & h_{24} \\
h_{32} & h_{33} & h_{34} \\
h_{42} & 0 & h_{44}
\end{array}\right), \quad X=\left(\begin{array}{c}
M \\
m_{4} \\
m
\end{array}\right), \quad 0=\left(\begin{array}{l}
0 \\
0 \\
0
\end{array}\right)
$$

satisfazendo $h_{11}=0$, o qual pode ser visto como um vínculo geométrico para as configurações.

Geometricamente, a equação (5.39) representa a interseção de três planos no espaço de massas $\left(M, m_{4}, m\right)$. Para termos soluções positivas de (5.39) o determinante de $H$ deve ser nulo. Por um cálculo direto temos

$$
\operatorname{det} H=h_{11} F
$$

onde $F$ é não nulo. Assim, sobre o nível zero de $h_{11}$ a equação (5.39) tem soluções não triviais. Temos, então, que analisar condições para soluções positivas. Afim de simplificar nossas análises vamos fixar o seguinte sistema de coordenadas

$$
\begin{gathered}
r_{1}=(1,0,0), \quad r_{2}=\left(-\frac{1}{2}, \frac{\sqrt{3}}{2}, 0\right), \quad r_{3}=\left(-\frac{1}{2},-\frac{\sqrt{3}}{2}, 0\right), \quad r_{4}=(0,0, \sqrt{2}), \\
r_{5}=(x, 0, z), \quad r_{6}=\left(-\frac{x}{2}, \frac{\sqrt{3} x}{2}, z\right), \quad r_{7}=\left(-\frac{x}{2},-\frac{\sqrt{3} x}{2}, z\right) .
\end{gathered}
$$

Nestas coordenadas $x>0$ é raio do círculo que contém $\mathcal{E}_{1}$ e $z \in \mathbb{R}$ é posição relativa ao plano que contém $r_{1}, r_{2}$ e $r_{3}$ e o plano que contém $\mathcal{E}$.

Vamos localizar nestas coordenadas o nível zero de $h_{11}$. Considere novamente a expressão para $h_{11}$

$$
h_{11}=\left(R_{15}-R_{45}\right) \Delta_{1475}+\left(R_{16}-R_{46}\right) \Delta_{1476}
$$

Usando as coordenadas acima estudemos o sinal de cada termo em $h_{11}$. O termo $R_{15}-R_{45}$ é negativo se $z>(\sqrt{2} / 4)(1+2 x)$, positivo se $z<(\sqrt{2} / 4)(1+2 x)$ e se anula sobre a reta $z=$ $(\sqrt{2} / 4)(1+2 x)$. O termo $\Delta_{1475}$ é negativo se $z>(\sqrt{2})(1-x)$, positivo se $z<(\sqrt{2})(1-x)$ e se anula sobre a reta $z=(\sqrt{2})(1-x)$. O termo $R_{16}-R_{46}$ é negativo se $z>(\sqrt{2} / 4)(1-x)$, positivo se $z<(\sqrt{2} / 4)(1-x)$ e se anula sobre a reta $z=(\sqrt{2} / 4)(1-x)$. O termo $\Delta_{1476}$ é negativo se 
$z>(\sqrt{2} / 2)(2+x)$, positivo se $z<(\sqrt{2} / 2)(2+x)$ e se anula sobre a reta $z=(\sqrt{2} / 2)(2+x)$. Assim as componentes conexas do conjunto $h_{11}^{-1}(0)$ estão restritas a conjunto $\mathcal{A}_{1} \cup \mathcal{A}_{2} \cup \mathcal{A}_{3}$, onde

$$
\begin{gathered}
\mathcal{A}_{1}=\left\{(x, z) \in \mathbb{R}^{2}: x>1, \sqrt{2}(1-x)<z<\frac{\sqrt{2}}{4}(1-x)\right\}, \\
\mathcal{A}_{2}=\left\{(x, z) \in \mathbb{R}^{2}: 0<x<1, \frac{\sqrt{2}}{4}(1-x)<z<\sqrt{2}(1-x)\right\}, \\
\mathcal{A}_{3}=\mathcal{A}_{31} \cup \mathcal{A}_{32},
\end{gathered}
$$

onde

$$
\begin{aligned}
& \mathcal{A}_{31}=\left\{(x, z) \in \mathbb{R}^{2}: 0<x \leq \frac{1}{2}, \sqrt{2}(1-x)<z<\frac{\sqrt{2}}{2}(2+x)\right\}, \\
& \mathcal{A}_{32}=\left\{(x, z) \in \mathbb{R}^{2}: x>\frac{1}{2}, \frac{\sqrt{2}}{4}(1+2 x)<z<\frac{\sqrt{2}}{2}(2+x)\right\} .
\end{aligned}
$$

Veja a figura 5.29.

É suficiente estudarmos a existência de configurações centrais sobre os conjuntos $\mathcal{A}_{1}$ e $\mathcal{A}_{3}$, pois os casos sobre o conjunto $\mathcal{A}_{2}$ foram considerados por Hampton e Santoprete em [14].

Para provar a existência de soluções positivas de (5.39) sobre o conjunto $h_{11}^{-1}(0)$ é suficiente mostrar que as entradas de cada linha da matriz $H$ trocam de sinais. Então, se as entradas de alguma das linhas de $H$ tem o mesmo sinal não existem massas admissíveis tais que os corpos estejam em configuração central de acordo com a figura 5.28. Temos o seguinte lema para $(x, z) \in \mathcal{A}_{1}$.

Lema 5.4.20 Sobre o conjunto

$$
\mathcal{A}_{1}=\left\{(x, z) \in \mathbb{R}^{2}: x>1, \sqrt{2}(1-x)<z<\frac{\sqrt{2}}{4}(1-x)\right\}
$$

as entradas $h_{22}, h_{23}$ e $h_{24}$ de $H$ tem os mesmos sinais. Por conseguinte, neste caso, não existem configurações centrais de acordo com a figura 5.28.

Prova: Considere a entrada $h_{22}=\left(R_{13}-R_{36}\right) \Delta_{1623}$. Para $z<0$ temos $\Delta_{1623}>0$ e sobre o conjunto $\mathcal{B}_{22}=\left\{\sqrt{2-x-x^{2}} \leq z<0\right\}$ a parcela $\left(R_{13}-R_{36}\right)$ é não positiva e estritamente positiva 


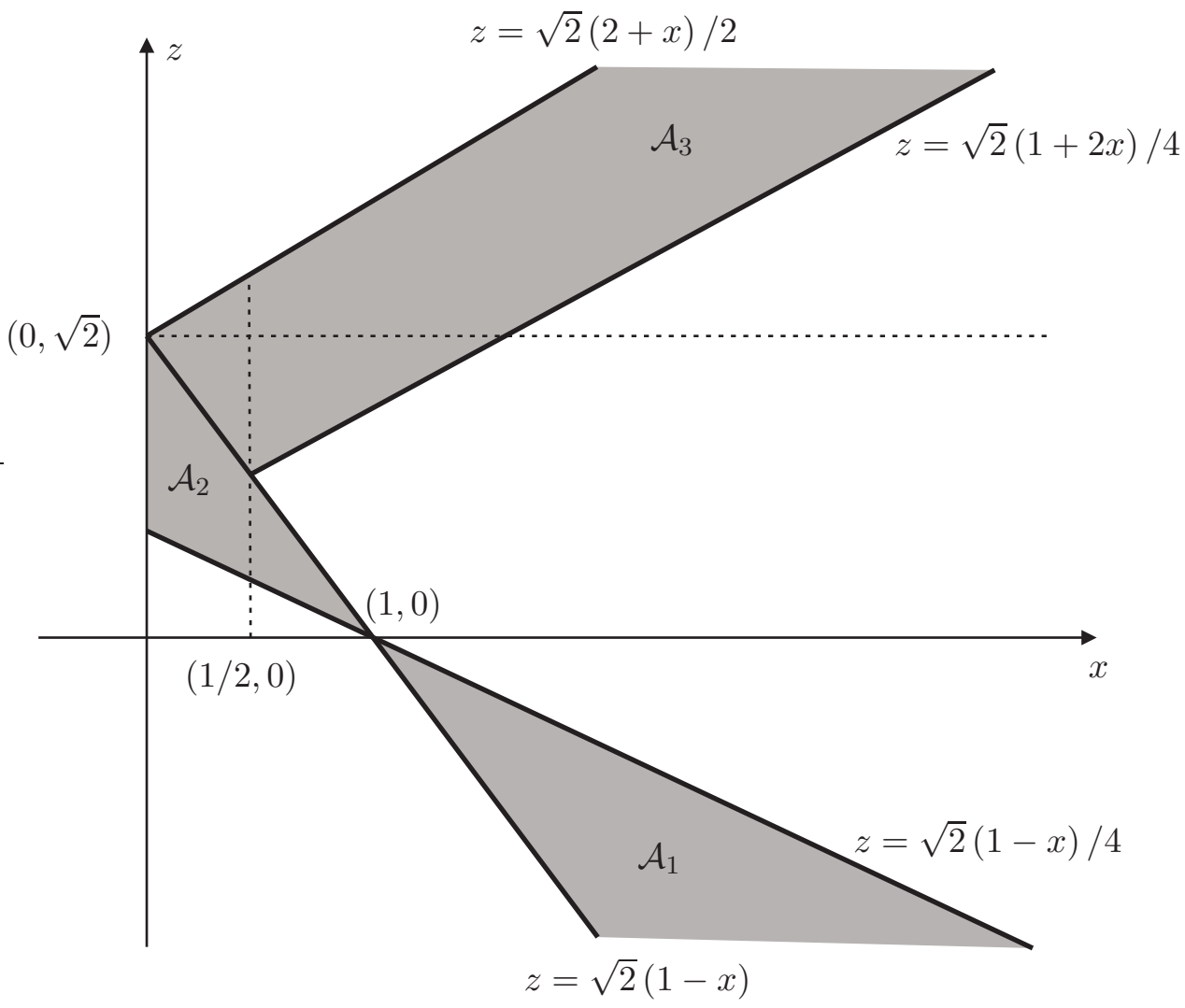

Figura 5.29: As regiões $\mathcal{A}_{1}, \mathcal{A}_{2}$ e $\mathcal{A}_{3}$.

fora de $\mathcal{B}_{22}$. Como $\mathcal{B}_{22} \cap \mathcal{A}_{1}=\emptyset$, o sinal de $h_{22}$ sobre $\mathcal{A}_{1}$ é sempre is positivo.

Considere a entrada $h_{23}=\left(R_{14}-R_{46}\right) \Delta_{1624}$. A parcela $\Delta_{1624}$ é positiva para $z<\sqrt{2}(1-x)$, negativa para $z>\sqrt{2}(1-x)$ e nula sobre a reta $z=\sqrt{2}(1-x)$. Assim, $\Delta_{1624}>0$ sobre o conjunto $\mathcal{A}_{1}$. Para valores $(x, z)$ no conjunto $\mathcal{B}_{23}=\left\{\sqrt{2}-\sqrt{3-x^{2}} \leq z<0\right\}$ a parcela $\left(R_{14}-R_{46}\right)$ é não positiva e estritamente positiva fora de $\mathcal{B}_{23}$. Como $\mathcal{B}_{23} \cap \mathcal{A}_{1}=\emptyset$, o sinal de $h_{23}$ sobre $\mathcal{A}_{1}$ é sempre positivo.

Considere a entrada $h_{24}=\left(R_{17}-R_{67}\right) \Delta_{1627}$. Para $z<0$ temos $\Delta_{1627}>0$ e sobre o conjunto $\mathcal{B}_{24}=\left\{z<\sqrt{2 x^{2}-x-1}\right\}$ a parcela $\left(R_{13}-R_{36}\right)$ é não positiva e estritamente positiva fora de $\mathcal{B}_{24}$. Como $\mathcal{B}_{24} \cap \mathcal{A}_{1}=\emptyset$, o sinal de $h_{24}$ sobre $\mathcal{A}_{1}$ é sempre positivo. Isto completa a prova do lema.

Provaremos analiticamente a existência de configurações centrais de acordo com a figura 5.28 para pontos no conjunto $\mathcal{A}_{3}$. 
Lema 5.4.21 Considere o conjunto

$$
\mathcal{A}_{32}=\left\{(x, z) \in \mathbb{R}^{2}: x>\frac{1}{2}, \frac{\sqrt{2}}{4}(1+2 x)<z<\frac{\sqrt{2}}{2}(2+x)\right\} .
$$

Então existe um intervalo não vazio $I \subset(0, \infty)$, no qual para cada $x_{0} \in I$ existe $z_{0}>\sqrt{2}$ com $P_{0}=\left(x_{0}, z_{0}\right) \in h_{11}^{-1}(0) \cap \mathcal{A}_{32}$ tal que com posições

$$
\begin{gathered}
r_{1}=(1,0,0), \quad r_{2}=\left(-\frac{1}{2}, \frac{\sqrt{3}}{2}, 0\right), \quad r_{3}=\left(-\frac{1}{2},-\frac{\sqrt{3}}{2}, 0\right), \quad r_{4}=(0,0, \sqrt{2}), \\
r_{5}=\left(x_{0}, 0, z_{0}\right), \quad r_{6}=\left(-\frac{x_{0}}{2}, \frac{\sqrt{3} x_{0}}{2}, z_{0}\right), \quad r_{7}=\left(-\frac{x_{0}}{2},-\frac{\sqrt{3} x_{0}}{2}, z_{0}\right)
\end{gathered}
$$

existem massas positivas $M=m_{1}=m_{2}=m_{3}, m_{4}$ e $m=m_{5}=m_{6}=m_{7}$, tais que os sete corpo formam uma configuração central espacial de acordo com a figura 5.28.

Prova: Como dissemos a matriz $H$ é singular sobre o conjunto $\left\{h_{11}=0\right\}$. Então, sobre este conjunto, existem soluções não triviais de (5.39). Para simplificar nossa análise vamos nos restringir ao seguinte subconjunto de $\mathcal{A}_{32}$

$$
\mathcal{D}=\left\{(x, z) \in \mathbb{R}^{2}: 2^{1 / 3}<x<\frac{3}{2}, \sqrt{2}<z \leq \sqrt{2+2 x-x^{2}}\right\}
$$

Veja a figura 5.30.

Sobre o conjunto $\mathcal{D}$ algumas entradas da matriz $H$ tem sinais fixos, os quais são: $h_{22}<0$, $h_{23}>0, h_{24}>0, h_{33}>0, h_{34}<0, h_{42}>0$ e $h_{44}<0$. Enquanto que o termo $h_{32}<0$ no interior de $\mathcal{D}$ e $h_{32}=0$ sobre $\mathcal{D} \cap\left\{z=\sqrt{2+2 x-x^{2}}\right\}$, ou seja, temos os seguintes sinais para as entradas de $H$ restritos conjunto $\mathcal{D}$

$$
H=\left(\begin{array}{lll}
- & + & + \\
* & + & - \\
+ & 0 & -
\end{array}\right) .
$$

Assim resta apenas mostrarmos que o nível zero de $h_{11}$ tem interseção com o conjunto $\mathcal{D}$. Vamos 


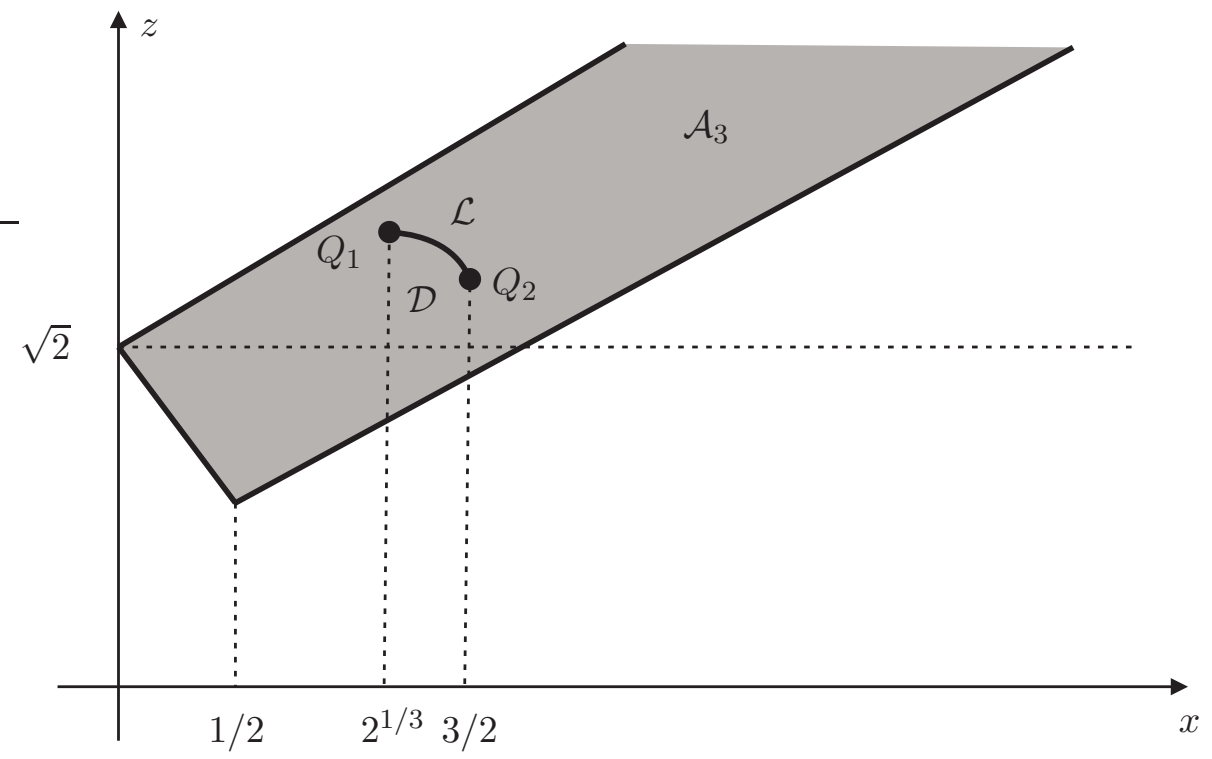

Figura 5.30: O conjunto $\mathcal{D}$ e os pontos $Q_{1}$ e $Q_{2}$.

considerar os termos que definem $h_{11}$ restritos ao bordo de $\mathcal{D}$. Mais precisamente, considere o conjunto

$$
\mathcal{L}=\mathcal{D} \cap\left\{z=\sqrt{2+2 x-x^{2}}\right\}
$$

veja a figura 5.30. É simples ver que $\mathcal{L}$ é um arco de circunferência fechado com pontos finais em

$$
Q_{1}=\left(2^{1 / 3}, \sqrt{2+2^{4 / 3}-2^{2 / 3}}\right), \quad Q_{2}=(3 / 2, \sqrt{11} / 2) .
$$

Avaliando a função $h_{11}$ nestes pontos temos,

$$
h_{11}\left(Q_{1}\right)=0.0862627114>0, \quad h_{11}\left(Q_{2}\right)=-0.2170428060<0 .
$$

Por conseguinte, existe um ponto $P_{0}=\left(x_{0}, z_{0}\right) \in \mathcal{L}$,

$$
x_{0} \in\left(2^{1 / 3}, 3 / 2\right), \quad z_{0}=\sqrt{2+2 x_{0}-x_{0}^{2}}
$$

tal que $h_{11}\left(P_{0}\right)=0$. Então, no ponto $P_{0}$ temos soluções não triviais positivas de (5.39), uma vez 
que os sinais das entradas de $H$ neste ponto são

$$
H\left(P_{0}\right)=\left(\begin{array}{ccc}
- & + & + \\
0 & + & - \\
+ & 0 & -
\end{array}\right)
$$

O intervalo $I$ contendo $x_{0}$ no qual para cada $x \in I$ existe $z>\sqrt{2}$, com $(x, z) \in h_{11}^{-1}(0) \cap \mathcal{A}_{32}$, para o qual temos soluções não triviais positivas de (5.39) é obtido pela continuidade das entradas da matriz $H$. A prova está completa.

Para darmos alguma informação acerca dos valores das massas nas posições correspondentes ao ponto $P_{0}=\left(x_{0}, z_{0}\right) \in \mathcal{L}$, considere

$$
M=m_{1}=m_{2}=m_{3}=1 .
$$

De avaliações numéricas temos

$$
x_{0}=1.3252606644270379418, \quad z_{0}=1.7012364621586273465,
$$

e assim,

$$
m_{4}=1.0030768074558921784, \quad m=m_{5}=m_{6}=m_{7}=1.3163213948824968524 .
$$

No próximo Capítulo faremos um caso de configuração central empilhada e colocaremos os problemas que são de interesse. 


\section{Capítulo 6}

\section{Configurações Centrais Empilhadas Parte 2}

Na primeira seção deste Capítulo responderemos a uma pergunta feita por Marshall Hampton em seu primeiro trabalho sobre configurações centrais empilhadas, veja o Teorema de Hampton em 5.4.1. A questão de Hampton, de 2005, está relacionada com a existência de uma configuração central empilhada de 5 massas, na qual um subconjunto de 4 massas também forme uma configuração central. Nesta época já era conhecido que, para cinco massas colineares com simetria adequada, tínhamos tal propriedade. Também era conhecido que, quatro massas iguais nos vértices de um quadrado e uma massa arbitrária na interseção das diagonais é uma configuração central empilhada, na qual poderíamos retirar a massa sobre a interseção das diagonais que ainda teríamos uma configuração central com quatro massas. Desta forma a pergunta de Hampton é a seguinte:

Além do caso colinear simétrico e do caso quadrado com massas iguais nos vértices e uma massa arbitrária na interseção das diagonais do quadrado, existe mais alguma configuração central empilhada de cinco massas na qual um subconjunto de quatro massas também forme uma configuração central?

\subsection{Configurações Centrais Planares, não Colineares, (5, 1)-Empilhadas}

A resposta à questão acima é não, para o caso planar, conforme o teorema seguinte. O único caso não colinear possível é um quadrado com massas iguais nos vértices e uma massa arbitrária na interseção das diagonais do quadrado. Vamos adotar aqui uma nomenclatura que definirá as configurações empilhadas. 
Definição 6.1.1 Uma configuração central será chamada de $(n, k)$-empilhada, se podemos remover $k$ massas, (as $k$ com maiores índices, por convenção), de tal forma que as $(n-k)$ massas restantes ainda formem uma configuração central.

Assim, Hampton em [13] e Mello e Llibre em [28] resolveram casos particulares de $(5,2)-$ empilhadas. Para responder à pergunta de Hampton, vamos estudar o caso de configuração central (5, 1)-empilhada no plano.

A resposta à pergunta de Hampton vem no seguinte resultado.

Teorema 6.1.2 Considere um problema planar não colinear de 5-corpos. Então, a única configuração central $(5,1)$-empilhada é formada pelo quadrado com massas iguais nos vértices e uma massa arbitrária na interseção das diagonais.

Prova: Usaremos aqui as equações de Andoyer com áreas. Dividiremos a prova em 5 lemas.

Considere 5 massas positivas, $\left(m_{1}, m_{2}, m_{3}, m_{4}, m_{5}\right)$ formando uma configuração central nas posições $\left(r_{1}, r_{2}, r_{3}, r_{4}, r_{5}\right)$, então pelo Teorema 5.2.4, as 10 equações de Andoyer com áreas devem ser satisfeitas. Seguem as equações

$$
\begin{aligned}
& f_{12}=m_{3}\left(R_{13}-R_{23}\right) \Delta_{123}+m_{4}\left(R_{14}-R_{24}\right) \Delta_{124}+m_{5}\left(R_{15}-R_{25}\right) \Delta_{125}=0, \\
& f_{13}=m_{2}\left(R_{12}-R_{32}\right) \Delta_{132}+m_{4}\left(R_{14}-R_{34}\right) \Delta_{134}+m_{5}\left(R_{15}-R_{35}\right) \Delta_{135}=0, \\
& f_{14}=m_{2}\left(R_{12}-R_{42}\right) \Delta_{142}+m_{3}\left(R_{13}-R_{43}\right) \Delta_{143}+m_{5}\left(R_{15}-R_{45}\right) \Delta_{145}=0, \\
& f_{15}=m_{2}\left(R_{12}-R_{52}\right) \Delta_{152}+m_{3}\left(R_{13}-R_{53}\right) \Delta_{153}+m_{4}\left(R_{14}-R_{54}\right) \Delta_{154}=0, \\
& f_{23}=m_{1}\left(R_{21}-R_{31}\right) \Delta_{231}+m_{4}\left(R_{24}-R_{34}\right) \Delta_{234}+m_{5}\left(R_{25}-R_{35}\right) \Delta_{235}=0, \\
& f_{24}=m_{1}\left(R_{21}-R_{41}\right) \Delta_{241}+m_{3}\left(R_{23}-R_{43}\right) \Delta_{243}+m_{5}\left(R_{25}-R_{45}\right) \Delta_{245}=0, \\
& f_{25}=m_{1}\left(R_{21}-R_{51}\right) \Delta_{251}+m_{3}\left(R_{23}-R_{53}\right) \Delta_{253}+m_{4}\left(R_{24}-R_{54}\right) \Delta_{254}=0, \\
& f_{34}=m_{1}\left(R_{31}-R_{41}\right) \Delta_{341}+m_{2}\left(R_{32}-R_{42}\right) \Delta_{342}+m_{5}\left(R_{35}-R_{45}\right) \Delta_{345}=0,
\end{aligned}
$$


6.1. CONFIGURAÇÕES CENTRAIS PLANARES, NÃO COLINEARES, (5, 1)-EMPILHADAS 125

$$
\begin{aligned}
& f_{35}=m_{1}\left(R_{31}-R_{51}\right) \Delta_{351}+m_{2}\left(R_{32}-R_{52}\right) \Delta_{352}+m_{4}\left(R_{34}-R_{54}\right) \Delta_{354}=0 \\
& f_{45}=m_{1}\left(R_{41}-R_{51}\right) \Delta_{451}+m_{2}\left(R_{42}-R_{52}\right) \Delta_{452}+m_{3}\left(R_{43}-R_{53}\right) \Delta_{453}=0 .
\end{aligned}
$$

Como estamos interessados no caso de configurações centrais $(5,1)$-empilhadas, depois de removido o corpo de massa $m_{5}$ e posição $r_{5}$, as massas $\left(m_{1}, m_{2}, m_{3}, m_{4}\right)$ ainda devem formar uma configuração central nas mesmas posições $\left(r_{1}, r_{2}, r_{3}, r_{4}\right)$. Então as equações de Andoyer para o caso de quatro massas também devem ser satisfeitas. Neste caso, representaremos as equações pela letra $g_{i j}$ para evitar ambiguidades com as equações acima. Assim seguem as equações,

$$
\begin{aligned}
& g_{12}=m_{3}\left(R_{13}-R_{23}\right) \Delta_{123}+m_{4}\left(R_{14}-R_{24}\right) \Delta_{124}=0, \\
& g_{13}=m_{2}\left(R_{12}-R_{32}\right) \Delta_{132}+m_{4}\left(R_{14}-R_{34}\right) \Delta_{134}=0, \\
& g_{14}=m_{2}\left(R_{12}-R_{42}\right) \Delta_{142}+m_{3}\left(R_{13}-R_{43}\right) \Delta_{143}=0, \\
& g_{23}=m_{1}\left(R_{21}-R_{31}\right) \Delta_{231}+m_{4}\left(R_{24}-R_{34}\right) \Delta_{234}=0, \\
& g_{24}=m_{1}\left(R_{21}-R_{41}\right) \Delta_{241}+m_{3}\left(R_{23}-R_{43}\right) \Delta_{243}=0, \\
& g_{34}=m_{1}\left(R_{31}-R_{41}\right) \Delta_{341}+m_{2}\left(R_{32}-R_{42}\right) \Delta_{342}=0 .
\end{aligned}
$$

Observe que, para alguns índices, $g_{i j}$ é parte da equação $f_{i j}$, no seguinte sentido,

$$
\begin{aligned}
& f_{12}=g_{12}+m_{5}\left(R_{15}-R_{25}\right) \Delta_{125}, \quad f_{13}=g_{13}+m_{5}\left(R_{15}-R_{35}\right) \Delta_{135}, \\
& f_{14}=g_{14}+m_{5}\left(R_{15}-R_{45}\right) \Delta_{145}, \quad f_{23}=g_{23}+m_{5}\left(R_{25}-R_{35}\right) \Delta_{235}, \\
& f_{24}=g_{24}+m_{5}\left(R_{25}-R_{45}\right) \Delta_{245}, \quad f_{34}=g_{34}+m_{5}\left(R_{35}-R_{45}\right) \Delta_{345} .
\end{aligned}
$$

Como $g_{12}=0, g_{13}=0, g_{14}=0, g_{23}=0, g_{24}=0$ e $g_{34}=0$, então $f_{12}=0, f_{13}=0, f_{14}=0$, $f_{23}=0, f_{24}=0$ e $f_{34}=0$ se, e somente se, todos os coeficientes envolvendo $m_{5}$ nas equações $f_{i j}$ acima se anulam, desde de que assumimos apenas os casos onde $m_{5}>0$. Por conseguinte, 
uma condição necessária para termos uma configuração central $(5,1)$-empilhada é que as seguintes equações sejam verificadas:

$$
\begin{aligned}
& \left(R_{15}-R_{25}\right) \Delta_{125}=0, \\
& \left(R_{15}-R_{35}\right) \Delta_{135}=0, \\
& \left(R_{15}-R_{45}\right) \Delta_{145}=0, \\
& \left(R_{25}-R_{35}\right) \Delta_{235}=0, \\
& \left(R_{25}-R_{45}\right) \Delta_{245}=0, \\
& \left(R_{35}-R_{45}\right) \Delta_{345}=0 .
\end{aligned}
$$

Com isto, provamos o seguinte lema.

Lema 6.1.3 Uma condição necessária para termos uma configuração central $(5,1)$-empilhada é que a configuração central com cinco massas não dependa do valor de $m_{5}$.

Considerando as equações (6.1)-(6.6) somos capazes de provar os seguintes lemas.

Lema 6.1.4 Em uma configuração central $(5,1)$-empilhada o quadrilátero formado pelas quatro massas que permanecem deve ser estritamente convexo.

Prova: Faremos a prova por contradição. Suponha que os corpos que permanecem formem um quadrilátero côncavo. Sem perda de generalidade, suponha que $r_{1}, r_{2}$ e $r_{3}$ estão nos vértices de um triângulo $\mathcal{T}$. Pelo teorema do perpendicular bissetor é claro que $r_{4}$ não pode pertencer a nenhum dos lados do triângulo. Assim, considere $r_{4}$ no interior do triângulo $\mathcal{T}$. Temos três casos a considerar: $r_{5}$ pertence ao exterior de $\mathcal{T} ; r_{5}$ pertence a algum lado de $\mathcal{T} ; r_{5}$ pertence ao interior de $\mathcal{T}$.

Caso 1: $r_{5}$ pertence ao exterior de $\mathcal{T}$. Suponha que $r_{5}$ pertença ao exterior de $\mathcal{T}$ sobre uma reta determinada por um lado de $\mathcal{T}$. Sem perda de generalidade, suponha que $r_{5}$ pertence à reta determinada pelo lado $\overline{r_{1} r_{2}}$, de acordo com a figura 6.1 (a). Segue que $\Delta_{135} \neq 0, \Delta_{145} \neq 0$ e 
$\Delta_{235} \neq 0$. Então, pelas equações (6.2), (6.3) e (6.4), temos $r_{15}=r_{25}=r_{35}=r_{45}=d_{1}>0$, que é $r_{1}, r_{2}, r_{3}$ e $r_{4}$ pertencem a um círculo de raio $d_{1}$ e centro em $r_{5}$. Mas, isto é uma contradição.

Agora considere o caso em que $r_{5}$ pertence ao exterior de $\mathcal{T}$, mas fora que qualquer reta que contenha um lado de $\mathcal{T}$. Sem perda de generalidade, considere $r_{5}$ no semiplano determinado pela reta que contém o lado $\overline{r_{1} r_{2}}$, de acordo com a figura 6.1 (b). Isto implica que $\Delta_{125} \neq 0, \Delta_{135} \neq 0$ e $\Delta_{235} \neq 0$. Então pelas equações (6.1), (6.2) e (6.4), temos $r_{15}=r_{25}=r_{35}=d_{2}>0$. Em outras palavras, $r_{1}, r_{2}$ e $r_{3}$ pertencem a um círculo de raio $d_{2}$ e centro em $r_{5}$. Usando $\Delta_{145} \neq 0$ ou $\Delta_{245} \neq 0$ em (6.5) temos $r_{45}=d_{2}$ e isto implica que $r_{4}$ também pertence ao círculo, mas isto é uma contradição, pois supusemos $r_{4}$ no interior de $\mathcal{T}$.

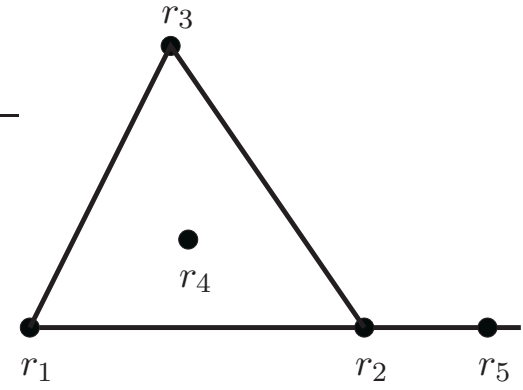

(a)

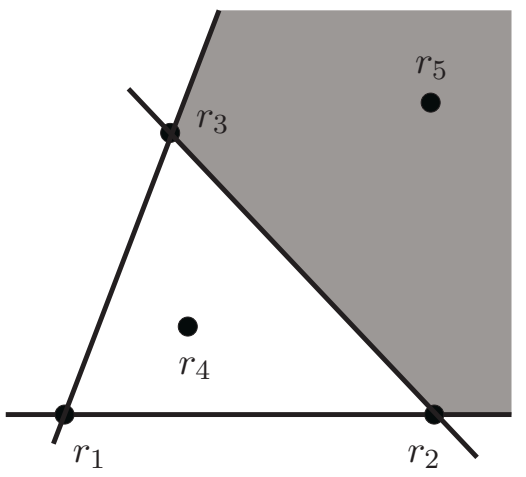

(b)

Figura 6.1: (a) $r_{1}, r_{2}$ e $r_{5}$ sobre uma reta. (b) $r_{5}$ no exterior de $\mathcal{T}$, mas fora das retas que contém algum dos lados de $\mathcal{T}$.

Caso 2: $r_{5}$ pertence a algum lado de $\mathcal{T}$. Sem perda de generalidade, considere $r_{5} \in \overline{r_{1} r_{2}}$. Isto implica que $\Delta_{135} \neq 0, \Delta_{145} \neq 0, \Delta_{235} \neq 0$ e $\Delta_{245} \neq 0$. Pelas equações (6.2), (6.3) e (6.4) temos $r_{15}=r_{25}=r_{35}=r_{45}=d_{3}>0$. Assim $r_{1}, r_{2}, r_{3}$ e $r_{4}$ pertencem a um círculo de raio $d_{3}$ e centro em $r_{5}$, o que é uma contradição.

Caso 3: $r_{5}$ pertence ao interior de $\mathcal{T}$. Isto implica que $\Delta_{125} \neq 0, \Delta_{135} \neq 0$ e $\Delta_{235} \neq 0$. Pelas equações (6.1), (6.2) e (6.4) temos $r_{15}=r_{25}=r_{35}=d_{4}>0$. Assim $r_{1}, r_{2}$ e $r_{3}$ pertencem a um círculo de raio $d_{4}$ e centro em $r_{5}$. Se $\Delta_{145} \neq 0$, pela equação (6.3), temos $r_{15}=r_{45}=d_{4}$. Desta forma $r_{4}$ pertence ao círculo, o que é uma contradição. Se $\Delta_{145}=0$, então $\Delta_{245} \neq 0$ a equação (6.5) nos dá $r_{25}=r_{45}=d_{4}$. Analogamente ao caso anterior temos uma contradição. 
Devido ao Lema 6.1.4, daqui por diante, assumiremos que os corpos que permanecem formam um quadrilátero convexo $\mathcal{Q}$.

Lema 6.1.5 Em uma configuração central (5,1)-empilhada o quinto corpo (a ser removido), não pode pertencer ao exterior do quadrilátero convexo $\mathcal{Q}$ formado pelos outros quatro corpos.

Prova: Faremos a prova por contradição. Sem perda de generalidade, suponha que $r_{1}, r_{2}, r_{3}$ e $r_{4}$ formam um quadrilátero convexo $\mathcal{Q}$ no qual os índices 1, 2, 3 e 4 estão colocados ciclicamente nos vértices. Consideremos dois casos: $r_{5}$ pertence ao exterior de $\mathcal{Q}$ sobre uma reta contendo um lado de $\mathcal{Q} ; r_{5}$ pertence ao exterior de $\mathcal{Q}$ fora de qualquer reta que contenha algum dos lados de $\mathcal{Q}$.

Caso 1: $r_{5}$ pertence ao exterior de $\mathcal{Q}$ sobre uma reta que contém um lado de $\mathcal{Q}$. Sem perda de generalidade, suponha que $r_{5}$ pertence à reta que contém o lado determinado por $\overline{r_{1} r_{2}}$, de acordo com a figura 6.2 (a). Então $\Delta_{135} \neq 0$ e $\Delta_{235} \neq 0$. Pelas equações (6.2) e (6.4), segue que $r_{15}=r_{25}$. Isto é uma contradição.

Caso 2: $r_{5}$ pertence ao exterior de $\mathcal{Q}$, mas fora de qualquer reta que contenha algum lado de $\mathcal{Q}$. Assim $\Delta_{125} \neq 0, \Delta_{135} \neq 0$ e $\Delta_{145} \neq 0$. Pelas equações (6.1), (6.2) e (6.3) temos $r_{15}=r_{25}=r_{35}=$ $r_{45}=d_{5}>0$. Por conseguinte, $r_{1}, r_{2}, r_{3}$ e $r_{4}$ pertencem a um círculo de raio $d_{5}$ e centro em $r_{5}$, de acordo com a figura 6.2 (b). Pelo teorema do perpendicular bissetor temos uma contradição.

Lema 6.1.6 Em uma configuração central (5,1)-empilhada o quinto corpo (a ser removido), não pode pertencer a nenhum dos lados do quadrilátero convexo $\mathcal{Q}$ formado pelos outros quatro corpos.

Prova: Sem perda de generalidade, suponha que $r_{1}, r_{2}, r_{3}$ e $r_{4}$ formam um quadrilátero convexo $\mathcal{Q}$ no qual os índices $1,2,3$ e 4 estão colocados ciclicamente nos vértices e $r_{5} \in \overline{r_{1} r_{2}}$. Assim $\Delta_{125}=0$ e $\Delta_{145} \neq 0$. Pela equação (6.3) temos $r_{15}=r_{45}$. Assim, $r_{5}$ pertence a reta perpendicular bissetora $\mathcal{B}$ relativa ao lado $\overline{r_{1} r_{4}}$, de acordo com a figura 6.3 , mas isto contradiz o teorema do perpendicular bissetor. 


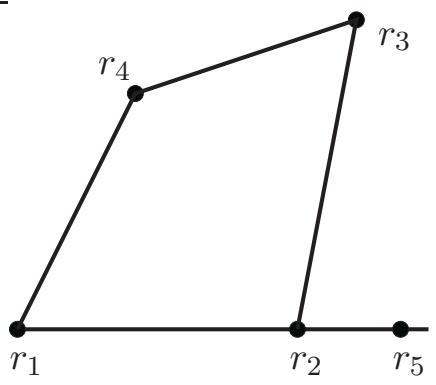

(a)

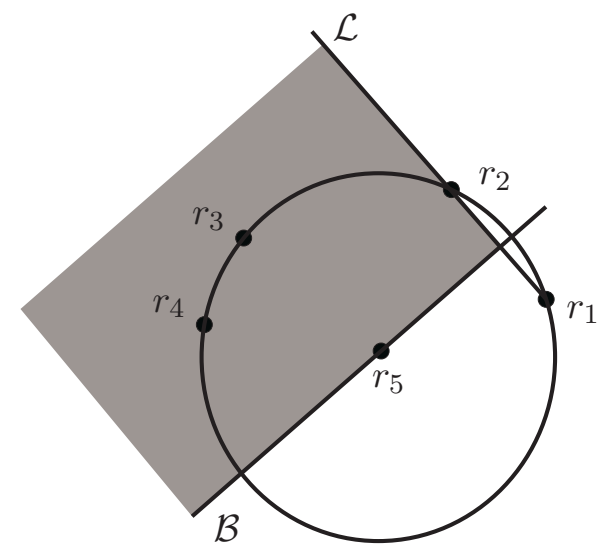

(b)

Figura 6.2: (a) $r_{1}, r_{2}$ e $r_{5}$ sobre uma reta. (b) $r_{1}, r_{2}, r_{3}$ e $r_{4}$ sobre um semicírculo com centro em $r_{5}$.

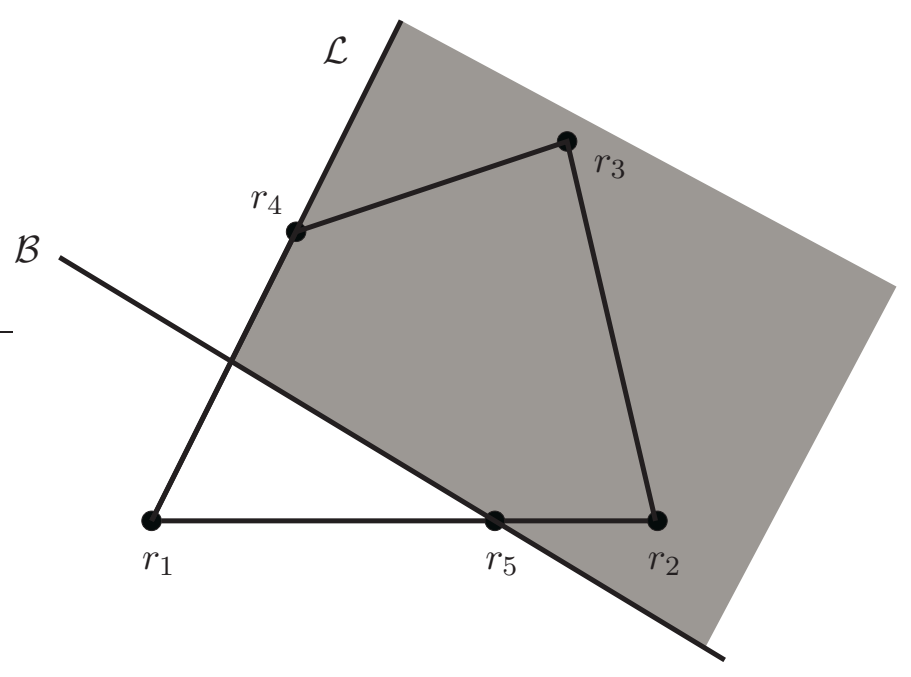

Figura 6.3: $r_{5} \in \overline{r_{1} r_{2}}$.

No próximo lema estudamos o caso onde $r_{1}, r_{2}, r_{3}$ e $r_{4}$ estão nos vértices de um quadrilátero convexo $\mathcal{Q}$ e o vetor posição $r_{5}$ está no interior de $\mathcal{Q}$.

Lema 6.1.7 Em uma configuração central $(5,1)$-empilhada se o quinto corpo (a ser removido) pertence ao interior do quadrilátero convexo $\mathcal{Q}$ formado pelos outros quatro corpos, então, a configuração central destes cinco corpos é um quadrado com quatro massas iguais em seus vértices e a massa $m_{5}$ na interseção das diagonais deste quadrado. 
Prova: Sem perda de generalidade, suponha que $r_{1}, r_{2}, r_{3}$ e $r_{4}$ formam um quadrilátero convexo $\mathcal{Q}$ no qual os índices 1, 2, 3 e 4 estão colocados ciclicamente nos vértices. Assuma que $r_{5}$ pertence ao interior de $\mathcal{Q}$. Assim $\Delta_{125} \neq 0, \Delta_{145} \neq 0, \Delta_{235} \neq 0$ e $\Delta_{345} \neq 0$. Pelas equações (6.1), (6.3), (6.4) e (6.6), temos $r_{15}=r_{25}=r_{35}=r_{45}=d_{6}>0$. Assim, $r_{1}, r_{2}, r_{3}$ e $r_{4}$ pertencem a um círculo de raio $d_{6}$ e centro em $r_{5}$, de acordo com a figura 6.4 .

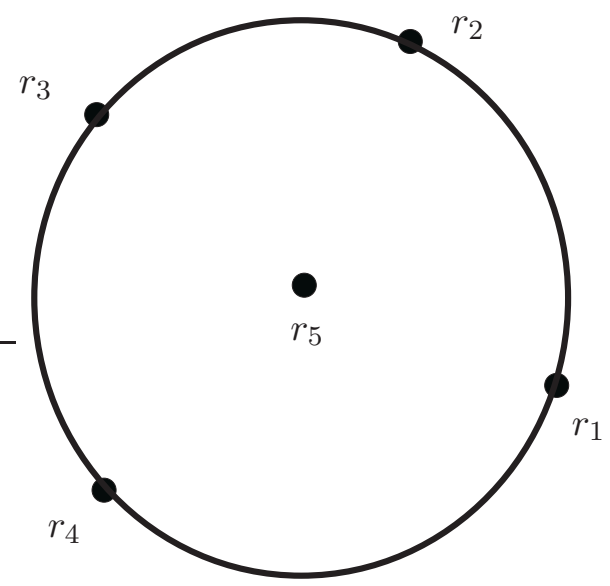

Figura 6.4: $r_{1}, r_{2}, r_{3}$ e $r_{4}$ sobre o círculo de raio $d_{6}$ e centro em $r_{5}$.

Vamos nos recordar outro resultado de Hampton, do Teorema 5.1.9. A única configuração central cocircular de quatro massas, com centro de massa no centro da circunferência, é formada por um quadrado com as massas iguais nos seus vértices.

Assim, para o nosso caso considerado basta mostrar que $r_{5}$ coincide com o centro de massa do sistema formado pelos outros quatro corpos $\mathcal{C}=\left(m_{1} r_{1}+m_{2} r_{2}+m_{3} r_{3}+m_{4} r_{4}\right) / \mathcal{M}, \mathcal{M}=$ $m_{1}+m_{2}+m_{3}+m_{4}$. Da definição de configuração central, definição 5.1.1, para o corpo de massa $m_{5}$, temos

$$
\lambda r_{5}=-\sum_{j=1}^{4} m_{j} \frac{r_{5}-r_{j}}{r_{j 5}^{3}}=-\sum_{j=1}^{4} m_{j} \frac{r_{5}-r_{j}}{d_{6}{ }^{3}} .
$$

A equação (6.7) é equivalente a

$$
d_{6}{ }^{3} \lambda r_{5}=\sum_{j=1}^{4} m_{j} r_{j}-\mathcal{M} r_{5}=\mathcal{M C}-\mathcal{M} r_{5}
$$


Pela definição de sistema baricêntrico, temos $\mathcal{M C}+m_{5} r_{5}=0$, que é, $\mathcal{M C}=-m_{5} r_{5}$. Substituindo isto na equação (6.8), temos

$$
\left(d_{6}{ }^{3} \lambda+M\right) r_{5}=0
$$

que deve se verificar para toda massa positiva $m_{5}$, veja o Lema 6.1.3. Suponha que $r_{5} \neq(0,0)$. Então, da equação (6.9), temos $d_{6}{ }^{3} \lambda+M=0$, para toda massa $m_{5}$. Assim,

$$
M+d_{6}{ }^{3} \lambda=M-d_{6}{ }^{3}\left(\frac{U}{I}\right)=M-d_{6}{ }^{3}\left[\frac{\sum_{1 \leq i<j \leq 5} \frac{m_{i} m_{j}}{r_{i j}}}{\sum_{i=1}^{5} m_{i}\left|r_{i}\right|^{2}}\right]=0,
$$

que deve se verificar para toda massa $m_{5}$. A equação (6.10) é equivalente a

$$
M \sum_{i=1}^{5} m_{i}\left|r_{i}\right|^{2}-d_{6}{ }^{3} \sum_{1 \leq i<j \leq 5} \frac{m_{i} m_{j}}{r_{i j}}=0
$$

para toda massa $m_{5}$. Por outro lado, a equação (6.11) é equivalente a

$$
\left|r_{5}\right|^{2} m_{5}^{2}+\left(\mathcal{M}\left(\left|r_{5}\right|^{2}-d_{6}{ }^{2}\right)+\sum_{i=1}^{4} m_{i}\left|r_{i}\right|^{2}\right) m_{5}-d_{6}{ }^{3} \sum_{1 \leq i<j \leq 4} \frac{m_{i} m_{j}}{r_{i j}}=0
$$

Note que a equação (6.12) é quadrática em termos da massa $m_{5}$ e isto implica que é verificada

para no máximo dois valores de $m_{5}$. É fácil ver que existem valores positivos de $m_{5}$, tais que a equação (6.12) não é satisfeita. Por conseguinte, a equação (6.9) é verificada para toda massa $m_{5}$ se, e somente se, $r_{5}=(0,0)$. Isto implica que $c=\mathcal{C}=(0,0)$.

Este lema completa a prova do Teorema 6.1.2.

\subsection{Configurações Centrais Espaciais (5,1)-Empilhadas}

A prova feita acima, mostra que no plano temos dois casos possíveis para configurações centrais $(5,1)$-empilhadas, a saber as configurações simétricas colineares e a configuração central formada 
por quatro massas iguais nos vértices de um quadrado com uma massa arbitrária no baricentro. Para o caso espacial temos duas possibilidades: uma configuração espacial de cinco massas que possui um subconjunto de quatro massas que forma uma configuração central espacial; e uma configuração central espacial que possui um subconjunto planar de quatro corpos que também forma uma configuração central, temos os seguintes resultados nos Teoremas 6.2.1 e 6.2.6.

Teorema 6.2.1 No caso espacial de 5-corpos, a única configuração central $(5,1)$-empilhada que possui um subconjunto espacial de quatro corpos que forma uma configuração central é o tetraedro regular com quatro massas iguais em seus vértices e uma massa arbitrária no baricentro.

Prova: Aqui usaremos as equações de Andoyer com volumes. Para o caso espacial com 5-corpos temos as seguintes

$$
\begin{aligned}
& f_{123}=m_{4}\left(R_{14}-R_{24}\right) \Delta_{1234}+m_{5}\left(R_{15}-R_{25}\right) \Delta_{1235}=0, \\
& f_{124}=m_{3}\left(R_{13}-R_{23}\right) \Delta_{1243}+m_{5}\left(R_{15}-R_{25}\right) \Delta_{1245}=0, \\
& f_{125}=m_{3}\left(R_{13}-R_{23}\right) \Delta_{1253}+m_{4}\left(R_{14}-R_{24}\right) \Delta_{1254}=0, \\
& f_{132}=m_{4}\left(R_{14}-R_{34}\right) \Delta_{1324}+m_{5}\left(R_{15}-R_{35}\right) \Delta_{1325}=0, \\
& f_{134}=m_{2}\left(R_{12}-R_{32}\right) \Delta_{1342}+m_{5}\left(R_{15}-R_{35}\right) \Delta_{1345}=0, \\
& f_{135}=m_{2}\left(R_{12}-R_{32}\right) \Delta_{1352}+m_{4}\left(R_{14}-R_{34}\right) \Delta_{1354}=0, \\
& f_{142}=m_{3}\left(R_{13}-R_{43}\right) \Delta_{1423}+m_{5}\left(R_{15}-R_{45}\right) \Delta_{1425}=0, \\
& f_{143}=m_{2}\left(R_{12}-R_{42}\right) \Delta_{1432}+m_{5}\left(R_{15}-R_{45}\right) \Delta_{1435}=0, \\
& f_{145}=m_{2}\left(R_{12}-R_{42}\right) \Delta_{1452}+m_{3}\left(R_{13}-R_{43}\right) \Delta_{1453}=0, \\
& f_{152}=m_{3}\left(R_{13}-R_{53}\right) \Delta_{1523}+m_{4}\left(R_{14}-R_{54}\right) \Delta_{1524}=0 \\
& f_{153}=m_{2}\left(R_{12}-R_{52}\right) \Delta_{1532}+m_{4}\left(R_{14}-R_{54}\right) \Delta_{1534}=0,
\end{aligned}
$$




$$
\begin{aligned}
& f_{154}=m_{2}\left(R_{12}-R_{52}\right) \Delta_{1542}+m_{3}\left(R_{13}-R_{53}\right) \Delta_{1543}=0, \\
& f_{231}=m_{4}\left(R_{24}-R_{32}\right) \Delta_{2314}+m_{5}\left(R_{25}-R_{35}\right) \Delta_{2315}=0, \\
& f_{234}=m_{1}\left(R_{21}-R_{31}\right) \Delta_{2341}+m_{5}\left(R_{25}-R_{35}\right) \Delta_{2345}=0, \\
& f_{235}=m_{1}\left(R_{21}-R_{31}\right) \Delta_{2351}+m_{4}\left(R_{24}-R_{34}\right) \Delta_{2354}=0, \\
& f_{241}=m_{3}\left(R_{23}-R_{43}\right) \Delta_{2413}+m_{5}\left(R_{25}-R_{45}\right) \Delta_{2415}=0, \\
& f_{243}=m_{1}\left(R_{21}-R_{41}\right) \Delta_{2431}+m_{5}\left(R_{25}-R_{45}\right) \Delta_{2435}=0, \\
& f_{245}=m_{1}\left(R_{21}-R_{41}\right) \Delta_{2451}+m_{3}\left(R_{23}-R_{43}\right) \Delta_{2453}=0, \\
& f_{251}=m_{3}\left(R_{23}-R_{53}\right) \Delta_{2513}+m_{4}\left(R_{24}-R_{54}\right) \Delta_{2514}=0, \\
& f_{253}=m_{1}\left(R_{21}-R_{51}\right) \Delta_{2531}+m_{4}\left(R_{24}-R_{54}\right) \Delta_{2534}=0, \\
& f_{254}=m_{1}\left(R_{21}-R_{51}\right) \Delta_{2541}+m_{3}\left(R_{23}-R_{53}\right) \Delta_{2543}=0, \\
& f_{341}=m_{2}\left(R_{32}-R_{42}\right) \Delta_{3412}+m_{5}\left(R_{35}-R_{45}\right) \Delta_{3415}=0, \\
& f_{342}=m_{1}\left(R_{31}-R_{41}\right) \Delta_{3421}+m_{5}\left(R_{35}-R_{45}\right) \Delta_{3425}=0, \\
& f_{345}=m_{1}\left(R_{31}-R_{41}\right) \Delta_{3451}+m_{2}\left(R_{32}-R_{42}\right) \Delta_{3452}=0, \\
& f_{351}=m_{2}\left(R_{32}-R_{52}\right) \Delta_{3512}+m_{4}\left(R_{34}-R_{54}\right) \Delta_{3514}=0, \\
& f_{352}=m_{1}\left(R_{31}-R_{51}\right) \Delta_{3521}+m_{4}\left(R_{34}-R_{54}\right) \Delta_{3524}=0, \\
& f_{354}=m_{1}\left(R_{31}-R_{51}\right) \Delta_{3541}+m_{2}\left(R_{32}-R_{52}\right) \Delta_{3542}=0, \\
& f_{451}=m_{2}\left(R_{42}-R_{52}\right) \Delta_{4512}+m_{3}\left(R_{43}-R_{53}\right) \Delta_{4513}=0, \\
& f_{452}=m_{1}\left(R_{41}-R_{51}\right) \Delta_{4521}+m_{3}\left(R_{43}-R_{53}\right) \Delta_{4523}=0, \\
& f_{453}=m_{1}\left(R_{41}-R_{51}\right) \Delta_{4531}+m_{2}\left(R_{42}-R_{52}\right) \Delta_{4532}=0 .
\end{aligned}
$$


Como estamos interessados no caso de configurações centrais $(5,1)$-empilhadas, depois de removido o corpo de massa $m_{5}$ e posição $r_{5}$, as massas $\left(m_{1}, m_{2}, m_{3}, m_{4}\right)$ ainda devem formar uma configuração central espacial, nas mesmas posições $\left(r_{1}, r_{2}, r_{3}, r_{4}\right)$. Então, as equações de Andoyer com volumes para o caso de quatro massas também devem ser satisfeitas. Neste caso, representaremos as equações pela letra $g_{i j k}$ para evitar ambiguidades com as equações acima. Assim seguem as equações,

$$
\begin{array}{ll}
g_{123}=m_{4}\left(R_{14}-R_{24}\right) \Delta_{1234}=0, & g_{124}=m_{3}\left(R_{13}-R_{23}\right) \Delta_{1243}=0, \\
g_{132}=m_{4}\left(R_{14}-R_{34}\right) \Delta_{1324}=0, & g_{134}=m_{2}\left(R_{12}-R_{32}\right) \Delta_{1342}=0, \\
g_{142}=m_{3}\left(R_{13}-R_{43}\right) \Delta_{1423}=0, & g_{143}=m_{2}\left(R_{12}-R_{42}\right) \Delta_{1432}=0, \\
g_{231}=m_{4}\left(R_{24}-R_{32}\right) \Delta_{2314}=0, & g_{234}=m_{1}\left(R_{21}-R_{31}\right) \Delta_{2341}=0, \\
g_{241}=m_{3}\left(R_{23}-R_{43}\right) \Delta_{2413}=0, & g_{243}=m_{1}\left(R_{21}-R_{41}\right) \Delta_{2431}=0, \\
g_{341}=m_{2}\left(R_{32}-R_{42}\right) \Delta_{3412}=0, & g_{342}=m_{1}\left(R_{31}-R_{41}\right) \Delta_{3421}=0 .
\end{array}
$$

Note que, analogamente ao caso planar, toda equação $f_{i j k}=0$, com $i \neq 5, j \neq 5$ e $k \neq 5$, é da forma

$$
f_{i j k}=g_{i j k}+m_{5}\left(R_{i 5}-R_{j 5}\right) \Delta_{i j k 5}=0 .
$$

Assim, temos como condição necessária para uma configuração central espacial $(5,1)$-empilhada as seguintes equações

$$
\left(R_{i 5}-R_{j 5}\right) \Delta_{i j k 5}=0
$$

para todos os $i, j$ e $k$, tais que $0<i<j<5, i \neq k \neq j$ e $k \neq 5$. Com estas considerações fica demonstrado o seguinte lema,

Lema 6.2.2 Uma condição necessária para termos uma configuração central $(5,1)$-empilhada é que a configuração central com cinco massas não dependa do valor de $m_{5}$. 
Recordando agora o Teorema 5.2.7 devido a Lemam-Filhès, que pode ser encontrado em [10], a única configuração central espacial de quatro corpos é o tetraedro regular com corpos de massas arbitrárias nos vértices. Assim, daqui por diante, nesta prova, os quatro corpos, cujos vetores posição e massas satisfazem as equações $g_{i j h}$, serão considerados nos vértices de um tetraedro regular $\mathcal{T}$. Temos os seguintes lemas.

Lema 6.2.3 Em uma configuração central espacial (5,1)-empilhada o quinto corpo (a ser removido), está no baricentro geométrico do tetraedro $\mathcal{T}$ formado pelos outros quatro corpos.

Prova: Suponha que $r_{1}, r_{2}, r_{3}$ e $r_{4}$ formam um tetraedro regular, então temos dois casos possíveis: $r_{5}$ está sobre algum plano contendo uma face de $\mathcal{T} ; r_{5}$ está fora de qualquer plano que contenha alguma das faces de $\mathcal{T}$.

Caso 1: Sem perda de generalidade, suponha que $r_{5}$ pertence ao plano determinado por $r_{1}, r_{2}$ e $r_{4}$. Então $\Delta_{1235} \neq 0, \Delta_{1345} \neq 0$ e $\Delta_{2345} \neq 0$. Assim pelas equações (6.13), temos

$$
\begin{aligned}
& \left(R_{15}-R_{25}\right) \Delta_{1235}=0 \Leftrightarrow\left(R_{15}-R_{25}\right)=0, \\
& \left(R_{15}-R_{35}\right) \Delta_{1345}=0 \Leftrightarrow\left(R_{15}-R_{35}\right)=0, \\
& \left(R_{25}-R_{45}\right) \Delta_{2345}=0 \Leftrightarrow\left(R_{25}-R_{45}\right)=0 .
\end{aligned}
$$

Segue que, $r_{15}=r_{25}=r_{35}=r_{45}$. Isto é uma contradição. Assim $r_{5}$ não pertence a nenhum plano que contenha alguma face.

Caso 2: Suponha que $r_{5}$ não pertence a nenhum plano que contenha alguma das faces. Então $\Delta_{1235} \neq 0, \Delta_{1345} \neq 0$ e $\Delta_{2345} \neq 0$. Assim pelas equações (6.13), temos

$$
\begin{aligned}
& \left(R_{15}-R_{25}\right) \Delta_{1235}=0 \Leftrightarrow\left(R_{15}-R_{25}\right)=0, \\
& \left(R_{15}-R_{35}\right) \Delta_{1345}=0 \Leftrightarrow\left(R_{15}-R_{35}\right)=0, \\
& \left(R_{25}-R_{45}\right) \Delta_{2345}=0 \Leftrightarrow\left(R_{25}-R_{45}\right)=0 .
\end{aligned}
$$


Segue que, $r_{15}=r_{25}=r_{35}=r_{45}$. Ou seja, $r_{5}$ está no baricentro geométrico de $\mathcal{T}$.

Lema 6.2.4 Uma condição suficiente para que uma configuração espacial de cinco corpos, formada por quatro corpos nos vértices de um tetraedro regular $\mathcal{T}$ e um corpo no baricentro geométrico de $\mathcal{T} e ́$

$$
m_{1}=m_{2}=m_{3}=m_{4} .
$$

Prova: Com as hipóteses do lema é fácil verificar que as seguintes equações de Andoyer se verificam

$$
\begin{aligned}
& f_{123}=f_{124}=f_{125}=f_{132}=f_{134}=f_{135}=0, \\
& f_{142}=f_{143}=f_{145}=f_{231}=f_{234}=f_{235}=0, \\
& f_{241}=f_{243}=f_{245}=f_{341}=f_{342}=f_{345}=0 .
\end{aligned}
$$

Restam apenas 12 equações da forma $f_{i 5 j}$, para todos os $0<i<5,0<j<5$ com $i \neq j \neq 5$. Podemos escrever estas equações na seguinte forma

$$
f_{i 5 j}=\left(m_{p}-m_{q}\right)\left(R_{i p}-R_{5 p}\right) \Delta_{i 5 j p}
$$

com $1 \leq p \leq 4,1 \leq q \leq 4$ com $q \neq p$, pertinentes as equações.

Das hipóteses do lema todos os volumes $\Delta i 5 j p$ são não nulos e também os termos $\left(R_{i p}-R_{5 p}\right)$. Assim, para que todas as equações em (6.14) se verifiquem é suficiente que $m_{p}=m_{q}$ se verifique para $1 \leq p \leq 4,1 \leq q \leq 4$ com $q \neq p$. Isto completa a prova do lema.

Com os resultados dos Lemas 6.2.2, 6.2.3 e 6.2.4 o teorema fica demonstrado. Vide figura 6.5. 


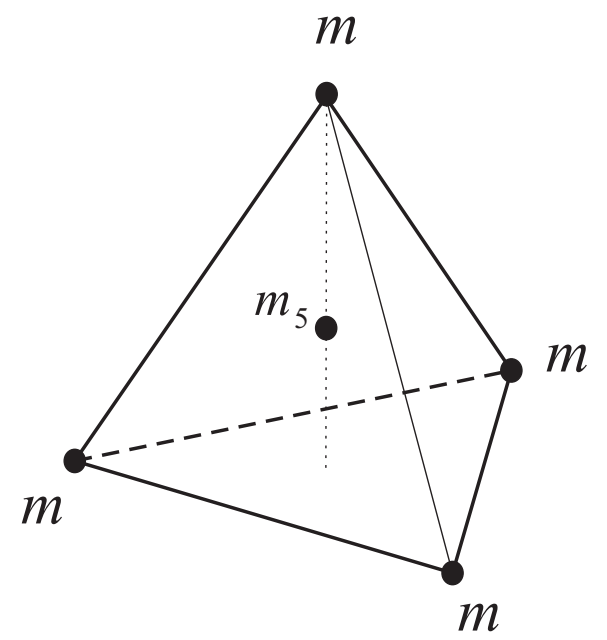

Figura 6.5: Configuração central $(5,1)$-empilhada que deixa uma configuração espacial.

Resta agora apenas o caso de uma configuração central espacial de cinco corpos que possui um subconjunto planar, que também é uma configuração central. Vamos precisar da seguinte definição, vide o trabalho de Fayçal em [8].

Definição 6.2.5 Uma configuração de $n$ corpos será chamada de configuração piramidal se for formada por $(n-1)$ corpos co-planares e um corpo fora deste plano. Vide figura 6.6. O corpo de maior índice é o do topo, por convenção.

Para este tipo de configuração ser uma configuração central $(5,1)$-empilhada temos algumas condições que são apresentadas no seguinte resultado.

Teorema 6.2.6 No caso espacial de 5-corpos, as únicas configurações centrais (5,1)-empilhadas que possuem um subconjunto planar de quatro corpos que forma uma configuração central são as configurações centrais piramidais.

Prova: Dividiremos a prova em três lemas.

Lema 6.2.7 Uma condição necessária para termos uma configuração central piramidal de 5-corpos é que o corpo no topo seja equidistante aos demais. Em particular, isto também implica que a configuração piramidal é independente do valor da massa $m_{5}$ do corpo colocado no topo. 


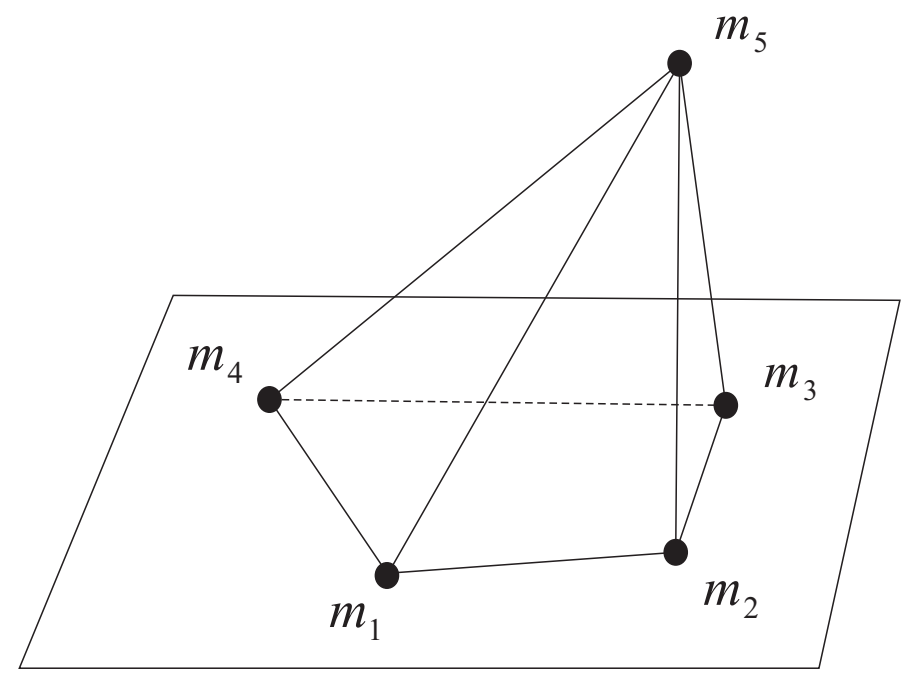

Figura 6.6: Configuração piramidal.

Prova: Consideremos as equações de Andoyer com volumes para o caso espacial de cinco corpos, são as mesmas listadas da prova do Teorema anterior. Considere as equações $f_{i j h}$, tais que os índices $0<i, j, h<5$. Com as considerações da definição 6.2 .5 todos os volumes que não envolvam o índice 5 serão nulos devido à coplanaridade dos corpos,

$$
\Delta_{i j h k}=0, \quad \forall 0<i, j, k, h<5 .
$$

Todos os volumes que envolvam o índice 5 serão não nulos.

$$
\Delta_{i j h 5} \neq 0, \quad \forall \quad 0<i, j, h<5,
$$

bem como para todas as permutações de $i, j, k$ e 5 em (6.15).

Assim para índices diferentes de 5 as equações de Andoyer se escrevem como

$$
f_{i j k}=m_{5}\left(R_{i 5}-R_{j 5}\right) \Delta_{i j h 5}=0
$$


como $\Delta_{i j h 5} \neq 0$ estas equações se verificam se, e só se, $R_{i 5}=R_{j 5}$. Varrendo todas as 12 possibilidades para combinações pertinentes destes índices, teremos

$$
r_{15}=r_{25}=r_{35}=r_{45}
$$

Em outras palavras, o corpo do topo é equidistante aos demais. Note que quando a igualdade acima é satisfeita todas as equações que envolvem a massa $m_{5}$ serão trivialmente satisfeitas, assim fica demonstrado o lema.

Assim uma figura que melhor representa uma configuração central piramidal de cinco corpos é formada por quatro corpos co-planares inscritos numa circunferência e um corpo fora do plano gerado por estes quatro passando pela reta perpendicular ao plano que contém o centro da circunferência, vide figura 6.7 .

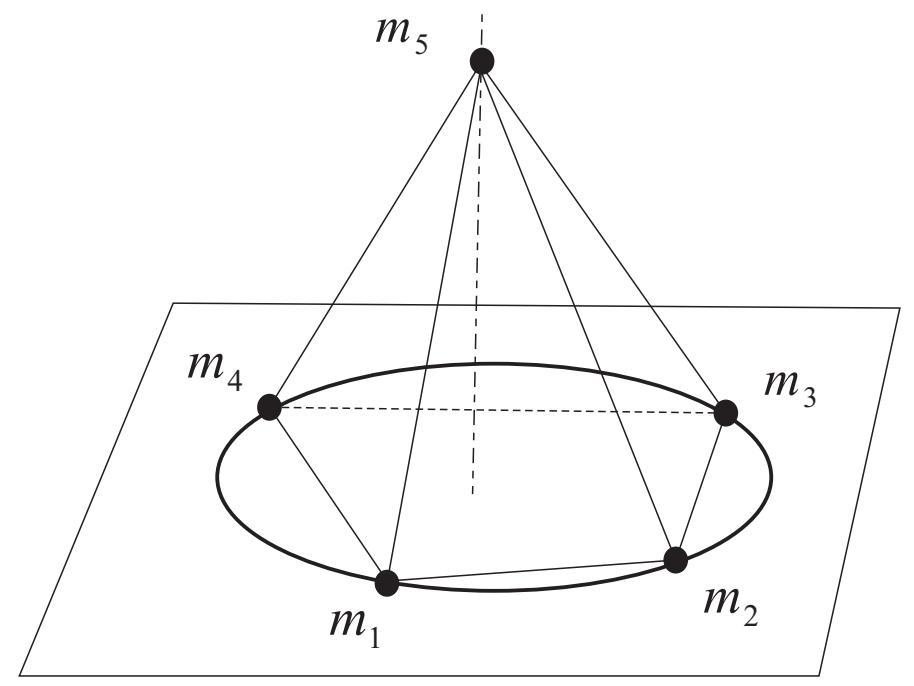

Figura 6.7: Configuração central $(5,1)$-empilhada que deixa uma configuração planar.

Para finalizar a prova do teorema vamos mostrar que toda configuração central piramidal de 5-corpos é também uma configuração central espacial $(5,1)$-empilhada. 
Lema 6.2.8 Considere uma configuração central espacial piramidal formada por 5-corpos, com $r_{5}$ no topo. Então, a configuração formada pelos corpos na base é uma configuração central planar.

Prova: Por hipótese, todas as 30 equações de Andoyer são satisfeitas. Como já vimos, as equações $f_{i j h}$, tais que os índices $0<i, j, k<5$ implicam que a configuração é independente do valor da massa $m_{5}$. Considere agora a seguinte

$$
f_{125}=m_{3}\left(R_{13}-R_{23}\right) \Delta_{1253}+m_{4}\left(R_{14}-R_{24}\right) \Delta_{1254}=0,
$$

note que os volumes envolvidos nesta equação são da forma

$$
\begin{aligned}
& \Delta_{1235}=\left(r_{1}-r_{2}\right) \wedge\left(r_{1}-r_{3}\right) \bullet\left(r_{1}-r_{5}\right), \\
& \Delta_{1245}=\left(r_{1}-r_{2}\right) \wedge\left(r_{1}-r_{4}\right) \bullet\left(r_{1}-r_{5}\right) .
\end{aligned}
$$

Como $r_{1}, r_{2}, r_{3}$ e $r_{4}$ são co-planares o produto vetorial entre quaisquer deles estará na direção de $r_{5}-\mathcal{O}$, onde $\mathcal{O}$ é o centro do círculo que contém $r_{1}, r_{2}, r_{3}$ e $r_{4}$. Lembrando que $\left\|r_{1}-r_{5}\right\| \neq 0$ e $\left\|r_{5}-\mathcal{O}\right\| \neq 0$, temos

$$
\begin{aligned}
& \Delta_{1235}=-\Delta_{123} \bullet\left(r_{5}-\mathcal{O}\right), \\
& \Delta_{1245}=-\Delta_{124} \bullet\left(r_{5}-\mathcal{O}\right) .
\end{aligned}
$$

Tendo em conta estas duas expressões podemos escrever $f_{125}=0$ na seguinte forma

$$
\left.f_{125}=m_{3}\left(R_{13}-R_{23}\right) \Delta_{123} \bullet\left(r_{5}-\mathcal{O}\right)+m_{4}\left(R_{14}-R_{24}\right) \Delta_{124} \bullet\left(r_{5}-\mathcal{O}\right)\right)=0 .
$$

Note que a equação (6.16) é verificada se. e somente se,

$$
f_{12}=m_{3}\left(R_{13}-R_{23}\right) \Delta_{123}+m_{4}\left(R_{14}-R_{24}\right) \Delta_{124}=0,
$$


que é uma das equações de Andoyer com áreas. Seguindo um procedimento análogo a este concluiremos que

$$
\begin{aligned}
& f_{125}=0 \leftrightarrow f_{12}=0, \\
& f_{135}=0 \leftrightarrow f_{13}=0, \\
& f_{145}=0 \leftrightarrow f_{14}=0, \\
& f_{235}=0 \leftrightarrow f_{23}=0, \\
& f_{245}=0 \leftrightarrow f_{24}=0, \\
& f_{345}=0 \leftrightarrow f_{34}=0 .
\end{aligned}
$$

Em outras palavras, se temos uma configuração central piramidal de cinco corpos os quatro corpos da base sempre estão em um configuração central planar e cocircular de quatro corpos.

Assim toda configuração central piramidal de cinco corpos é uma configuração central $(5,1)$ empilhada que após retirarmos o corpo de massa $m_{5}$ do topo nos deixa uma configuração central planar. Findamos assim a prova do teorema.

Em verdade, dada uma configuração central planar cocircular de quatro corpos existe uma única posição possível para o corpo de massa $m_{5}$, tal que os cinco corpos formem uma configuração central piramidal, vide [8]. Outros trabalhos que podem interessar ao leitor na linha das configurações centrais piramidais são de Zang e Zhou em [48] e de Mello e Fernandes em [23].

\subsection{Casos de Configurações Centrais $(n, 1)$-Empilhadas}

Como é sabido, vide o Teorema 5.3.1, no caso de uma configuração central planar não colinear $(4,1)$-empilhada a única possibilidade é: três corpos de massas iguais nos vértices de um triângulo equilátero, que é um polígono regular inscritível de três lados, e uma massa arbitrária no baricentro 
deste polígono regular. Na última seção vimos que este resultado também vale para o caso de configuração central planar não colinear $(5,1)$-empilhada. Em outras palavras, a única possibilidade é: um polígono regular com quatro corpos de massas iguais em seus vértices e uma massa arbitrária no baricentro deste polígono. Uma pergunta que surge naturalmente é se para o caso de configurações centrais planares não colineares valeria um resultado análogo? Existem algumas evidências de que a resposta seja afirmativa, embora não exista ainda uma prova completa.

Vamos enunciar um teorema no qual faremos uso da seguinte conjectura.

Conjectura 6.3.1 Considere $n$ corpos, com $n \geq 4$, numa configuração cocircular, então se o centro de massa coincide com o centro da circunferência a única configuração central possível é formada por $n$ corpos de massas iguais nos vértices de um polígono regular.

O resultado para quatro corpos, conforme citado acima está presente no trabalho de Hampton, vide a referência [11]. O caso geral ainda permanece em aberto.

Teorema 6.3.2 Considere que seja válida a conjectura 6.3.1, então a única configuração central planar não colinear, $(n, 1)$-empilhada de $n$ corpos, com $n \geq 5$, é formada por $(n-1)$ corpos de massas iguais nos vértices de um polígono regular e uma massa arbitrária no baricentro geométrico deste polígono.

Prova: Como nos casos anteriores as equações de Andoyer $f_{i j}=0$, devem ser satisfeitas pelos $n$ corpos. Considere então, as equações de Andoyer tais que $i \neq n$ e $j \neq n$, podemos escrevê-las da seguinte forma

$$
f_{i j}=\sum_{k \neq i, j, n} m_{k}\left(R_{i k}-R_{j k}\right) \Delta_{i j k}+m_{n}\left(R_{i n}-R_{j n}\right) \Delta_{i j n}
$$

para todos os índices $i$ e $j$, tais que $0<i<j<n$. Note que na equação acima a parte sob o somatório é a equação de Andoyer $g_{i j}=0$ para $(n-1)$ corpos, que também deve ser satisfeita para todos os índices $i$ e $j$, tais que $0<i<j<n$. Assim, como consideramos $m_{n}>0$, as seguintes equações são condições necessárias para termos uma configuração central $(n, 1)$-empilhada,

$$
\left(R_{i n}-R_{j n}\right) \Delta_{i j n}=0
$$


para todos os índices $i$ e $j$, tais que $0<i<j<n$.

Recorde que nas equações $f_{\text {in }}$ não figura a presença de $m_{n}$, com isto provamos o seguinte lema,

Lema 6.3.3 Uma condição necessária para termos uma configuração central $(n, 1)$-empilhada é que a configuração central com $n$ corpos não dependa do valor de $m_{n}$.

Vamos estudar agora as implicações das equações (6.17) na geometria das configurações, primeiro um lema para mostrar que a configuração dos $(n-1)$ corpos devem estar inscrita numa circunferência com centro em $r_{n}$. Em seguida um lema para mostrar que o centro de massa dos $(n-1)$ corpos deve coincidir com o centro da circunferência que os contém.

Lema 6.3.4 Uma condição necessária para termos uma configuração central $(n, 1)$-empilhada é que os $(n-1)$ corpos sejam cocirculares.

Prova: Como por hipótese os corpos são não colineares, nas equações (6.17) pelo menos um dos $\Delta_{i j n}$ é não nulo. Considere sem perda de generalidade que $\Delta_{12 n}$ seja não nulo, então a equação

$$
\left(R_{1 n}-R_{2 n}\right) \Delta_{12 n}=0 \leftrightarrow\left(R_{1 n}-R_{2 n}\right)=0,
$$

o que implica que $r_{1 n}=r_{2 n}=d>0$. Assim, $r_{1}$ e $r_{2}$ pertencem a uma circunferência de raio $d$ com centro em $r_{n}$.

Podemos separar os corpos restantes em dois conjuntos,

$$
\mathfrak{C}_{1}=\left\{j: \Delta_{1 j n}=0\right\}
$$

e

$$
\mathfrak{C}_{2}=\left\{j: \Delta_{1 j n} \neq 0\right\}
$$

Em outras palavras, $\mathfrak{C}_{1}$ representa os índices dos corpos cujos vetores posição são colineares com $r_{1}$ e $r_{n}$, enquanto que $\mathfrak{C}_{2}$ representa os índices dos corpos cujos vetores posição são não colineares 
com $r_{1}$ e $r_{n}$. Claramente para todo $j \in \mathfrak{C}_{2}$, temos o seguinte

$$
\left(R_{1 n}-R_{j n}\right) \Delta_{1 j n}=0 \leftrightarrow\left(R_{1 n}-R_{j n}\right)=0,
$$

ou seja, $r_{j n}=r_{1 n}=d>0$ para todo $j \in \mathfrak{C}_{2}$. Assim, $r_{1}, r_{2}$ e $r_{j}$ pertencem a uma circunferência de raio $d$ com centro em $r_{n}$, para todo $j \in \mathfrak{C}_{2}$.

Para completar a prova do lema vamos mostrar que $\mathfrak{C}_{1}$ possui no máximo um elemento. Suponha, por contradição, que existam dois índices $b, c \in \mathfrak{C}_{1}$, então $\Delta_{1 b n}=0$ o que implica que $\Delta_{2 b n} \neq 0, \log 0$

$$
\left(R_{2 n}-R_{b n}\right) \Delta_{2 b n}=0 \leftrightarrow\left(R_{2 n}-R_{b n}\right)=0,
$$

ou seja, $r_{b n}=r_{2 n}=d>0$, assim, $r_{b}$ pertence a uma circunferência de raio $d$ com centro em $r_{n}$, como nossas configurações são consideradas fora do conjunto colisão $r_{b}$ não pode coincidir com $r_{1}$, $\operatorname{logo} r_{b}$ deve ser diametralmente oposto a $r_{1}$. Considere agora o índice $c$, então $\Delta_{1 c n}=0$ o que implica que $\Delta_{2 c n} \neq 0, \operatorname{logo}$

$$
\left(R_{2 n}-R_{c n}\right) \Delta_{2 c n}=0 \leftrightarrow\left(R_{2 n}-R_{c n}\right)=0,
$$

ou seja, $r_{c n}=r_{2 n}=d>0$, mas isto é uma contradição. Pois, neste caso $r_{c}$ deveria coincidir com $r_{1}$ ou $r_{b}$. Vide figura 6.8. Assim terminamos a prova do lema.

Em particular, o último lema também implica que o polígono formado pelo vetores posição dos $(n-1)$ corpos é convexo, como no caso provado acima.

Passemos ao lema que trata da posição do centro de massas dos $(n-1)$ corpos. Já consideraremos no enunciado que os $(n-1)$ corpos estão sobre uma circunferência centrada em $r_{n}$.

Lema 6.3.5 Considere uma configuração central de $n$ corpos, com $n \geq 5$, formada por $(n-1)$ corpos cocirculares e uma massa arbitrária posicionada no centro da circunferência. Então, o centro de massa dos corpos cocirculares coincide com o centro da circunferência. 


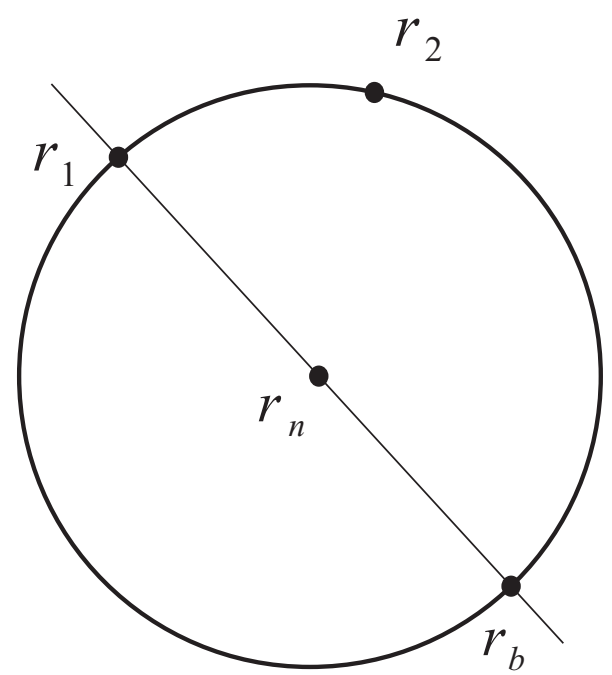

Figura 6.8: Não há posição para $r_{c}$ fora do conjunto colisão.

Prova: Sem perda de generalidade consideraremos que os vetores posição $r_{i}$ dos corpos cocirculares formam um polígono inscrito numa circunferência de raio $d$, no qual os índices 1 até $n-1$ estão dispostos ciclicamente e o corpo no centro da circunferência com índice $n$. Para os $n$ corpos vamos chamar de $\mathcal{M}_{n}$ a massa total e $\mathcal{C}_{n}$ o centro de massa. Para os $(n-1)$ corpos vamos chamar de $\mathcal{M}_{n-1}$ a massa total e $\mathcal{C}_{n-1}$ o centro de massa.

Assim tomando a equação de configurações centrais para o corpo de massa $m_{n}$ temos

$$
\lambda r_{n}=-\sum_{j=1}^{n-1} m_{j} \frac{r_{n}-r_{j}}{r_{j n}^{3}}=-\sum_{j=1}^{n-1} m_{j} \frac{r_{n}-r_{j}}{d^{3}} .
$$

A equação (6.18) é equivalente a

$$
d^{3} \lambda r_{n}=\sum_{j=1}^{n-1} m_{j} r_{j}-\mathcal{M}_{n-1} r_{n}=\mathcal{M}_{n-1} \mathcal{C}_{n-1}-\mathcal{M}_{n-1} r_{n}
$$

Pela definição de sistema baricêntrico, temos $\mathcal{M}_{n-1} \mathcal{C}_{n-1}+m_{n} r_{n}=0$, que é, $\mathcal{M}_{n-1} \mathcal{C}_{n-1}=-m_{n} r_{n}$. Substituindo isto na equação (6.19), temos

$$
\left(d^{3} \lambda+\mathcal{M}_{n}\right) r_{n}=0
$$


que deve se verificar para toda massa positiva $m_{n}$. Suponha, por contradição, que $r_{n} \neq(0,0)$. Então, da equação (6.20), temos $d^{3} \lambda+\mathcal{M}_{n}=0$, para toda massa $m_{n}$. Assim,

$$
\mathcal{M}_{n}+d^{3} \lambda=\mathcal{M}_{n}-d^{3}\left(\frac{U}{I}\right)=\mathcal{M}_{n}-d^{3}\left[\frac{\sum_{1 \leq i<j \leq n} \frac{m_{i} m_{j}}{r_{i j}}}{\sum_{i=1}^{n} m_{i}\left|r_{i}\right|^{2}}\right]=0
$$

que deve se verificar para toda massa $m_{n}$. A equação (6.21) é equivalente a

$$
\mathcal{M}_{n} \sum_{i=1}^{n} m_{i}\left|r_{i}\right|^{2}-d^{3} \sum_{1 \leq i<j \leq n} \frac{m_{i} m_{j}}{r_{i j}}=0
$$

para toda massa $m_{n}$. Por outro lado, a equação (6.22) é equivalente a

$$
\left|r_{n}\right|^{2} m_{n}^{2}+\left(\mathcal{M}_{n-1}\left(\left|r_{n}\right|^{2}-d^{2}\right)+\sum_{i=1}^{n-1} m_{i}\left|r_{i}\right|^{2}\right) m_{n}-d^{3} \sum_{1 \leq i<j \leq n-1} \frac{m_{i} m_{j}}{r_{i j}}=0
$$

Note que a equação (6.23) é quadrática em termos da massa $m_{n}$ e isto implica que é verificada para no máximo dois valores de $m_{n}$. É fácil ver que existem valores positivos de $m_{n}$, tais que a equação (6.23) não é satisfeita. Por conseguinte, a equação (6.20) é verificada para toda massa $m_{n}$ se, e somente se, $r_{n}=(0,0)$. Isto implica que $\mathcal{C}_{n-1}=\mathcal{C}_{n}=(0,0)$. Isto completa a prova do lema.

Com os resultados dos Lemas 6.3.3, 6.3.4, 6.3.5 e supondo a validade da Conjectura 6.3.1 o Teorema 6.3.2 fica demonstrado.

Para o caso espacial de configurações centrais $(n, 1)$-empilhadas temos o seguinte resultado,

Teorema 6.3.6 As únicas configurações centrais espaciais $(n, 1)$-empilhadas são formadas por $(n-1)$ corpos coesféricos e uma massa arbitrária no centro da esfera que contém os demais.

Prova: Como nos casos anteriores as equações de Andoyer $f_{i j l}=0$, devem ser satisfeitas pelos $n$ corpos. Considere então, as equações de Andoyer tais que $i \neq n, j \neq n$ e $l \neq n$, podemos escrevê-las 
da seguinte forma

$$
f_{i j l}=\sum_{k \neq i, j, l, n} m_{k}\left(R_{i k}-R_{j k}\right) \Delta_{i j l k}+m_{n}\left(R_{i n}-R_{j n}\right) \Delta_{i j l n}=0
$$

para todos os índices $i, j$ e $l$, pertinentes as equações de Andoyer. Note que na equação acima a parte sob o somatório é a equação de Andoyer $g_{i j l}=0$ para $(n-1)$ corpos, que também deve ser satisfeita para todos os índices pertinentes as equações de Andoyer. Assim, como consideramos $m_{n}>0$, as seguintes equações são condições necessárias para termos uma configuração central espacial $(n, 1)$-empilhada,

$$
\left(R_{i n}-R_{j n}\right) \Delta_{i j l n}=0
$$

para todos os índices $i, j$ e $l$, tais que $0<i<j<n, i \neq l \neq j$ e $l \neq n$.

Recorde que nas equações $f_{i j l}$, com $j=n$ ou $l=n$ não figura a presença de $m_{n}$, com isto provamos o seguinte lema.

Lema 6.3.7 Uma condição necessária para termos uma configuração central $(n, 1)$-empilhada é que a configuração central com $n$ corpos não dependa do valor de $m_{n}$.

Vamos estudar agora as implicações das equações (6.24) na geometria das configurações. Temos o seguinte lema.

Lema 6.3.8 Uma condição necessária para termos uma configuração central espacial $(n, 1)$-empilhada é que os $(n-1)$ corpos sejam coesféricos.

Prova: Como por hipótese os corpos são não coplanares, nas equações (6.24) pelo menos um dos $\Delta_{i j l n}$ é não nulo. Considere sem perda de generalidade que $\Delta_{123 n}$ seja não nulo, então a equação

$$
\left(R_{1 n}-R_{2 n}\right) \Delta_{123 n}=0 \leftrightarrow\left(R_{1 n}-R_{2 n}\right)=0
$$

o que implica que $r_{1 n}=r_{2 n}=d>0$. Assim, $r_{1}$ e $r_{2}$ pertencem a uma esfera de raio $d$ com centro em $r_{n}$. 
Podemos separar os corpos restantes em dois conjuntos,

$$
\mathfrak{D}_{1}=\left\{j: \Delta_{12 j n}=0\right\}
$$

e

$$
\mathfrak{D}_{2}=\left\{j: \Delta_{12 j n} \neq 0\right\}
$$

Em outras palavras, $\mathfrak{D}_{1}$ representa os índices dos corpos cujos vetores posição são coplanares com $r_{1}, r_{2}$ e $r_{n}$, enquanto que $\mathfrak{D}_{2}$ representa os índices dos corpos cujos vetores posição são não coplanares com $r_{1}, r_{2}$ e $r_{n}$. Claramente para todo $j \in \mathfrak{D}_{2}$, temos o seguinte

$$
\left(R_{1 n}-R_{j n}\right) \Delta_{12 j n}=0 \leftrightarrow\left(R_{1 n}-R_{j n}\right)=0
$$

ou seja, $r_{j n}=r_{1 n}=d>0$ para todo $j \in \mathfrak{C}_{2}$. Assim, $r_{1}, r_{2}$ e $r_{j}$ pertencem a uma esfera de raio $d$ com centro em $r_{n}$, para todo $j \in \mathfrak{D}_{2}$.

Para completar a prova do lema vamos notar que o índice 3 não pertence ao conjunto $\mathfrak{D}_{1}$, assim $r_{3 n}=d$. Tome agora equações de (6.24) para os quais o primeiro índice é 3 e o segundo índice esteja em $\mathfrak{D}_{1}$, assim

$$
\left(R_{3 n}-R_{j n}\right) \Delta_{3 j l n}=0 \leftrightarrow\left(R_{3 n}-R_{j n}\right)=0,
$$

donde, $r_{j n}=r_{3 n}=d>0$ para todo $j \in \mathfrak{D}_{1}$, ou seja, $r_{j n}=d>0$ para todo $j$, com $0<j<n$. Assim, terminamos a prova do lema.

Muito embora os dois últimos resultados no dêem caracterizações da configurações centrais $(n, 1)$-empilhadas seu entendimento completo ainda carece de estudo. Por exemplo, no caso da configuração central espacial $(5,1)$-empilhada vimos que o único caso que deixaria uma configuração central planar após a retirada do corpo de massa $m_{5}$ é uma configuração piramidal, que tem na base uma configuração central cocircular de 4 corpos. Mas, quais são as possibilidades de configurações centrais cocirculares de quatro corpos? Existem dois trabalhos que tratam deste problema, vide [30], 
no qual Mello e Fernandes fazem uma abordagem sobre configurações centrais do tipo pipa que são inscritíveis e vide [44] no qual Roberts e Cors fazem um estudo extenso sobre as propriedades das configurações centrais cocirculares de quatro corpos. Para o caso geral de configuração central planar cocircular o problema está completamente aberto, a menos da existência das configurações centrais formadas por massas iguais nos vértices de polígonos regulares. O caso das configurações centrais espaciais coesféricas também permanece em aberto.

\section{4 "Variação" de uma massa numa Configuração Central}

Dos resultados acima, vimos que as configurações centrais $(n, 1)$-empilhadas tem a propriedade de que podemos variar continuamente o valor da massa do corpo a ser removido mantendo as mesmas posições e os valores das outras massas fixos, que ainda teremos uma configuração central.

Uma questão colocada pelo matemático Alain Albouy, no XV Colóquio Brasileiro de Dinâmica Orbital, é a seguinte:

Que propriedades deve ter uma configuração central de $n$ corpos para que seja possível variar o valor da massa de um único corpo, mantendo fixos os valores das massas dos demais corpos e todas as posições e ainda ter uma configuração central?

Uma condição suficiente para que isto ocorra pode ser vista nas provas dos Teoremas 6.3.2 e 6.3.6, que é, nas equações de Andoyer, todos os coeficientes envolvendo a massa do corpo que queremos variar serem nulos. Uma questão natural é: existem outras configurações centrais com esta propriedade? A resposta é não e vem dada no seguinte resultado,

Teorema 6.4.1 Considere uma configuração central planar não colinear formada por $n$ corpos. Se podemos variar continuamente uma das massas mantendo as posições e demais massas fixas, então a configuração é uma configuração central planar $(n, 1)$-empilhada.

Prova: Sem perda de generalidade vamos supor que a massa que pode ser variada é a de índice $n, m_{n}$. Considere dois valores diferentes para esta massa $m_{n}=\mathcal{A}_{n}$ e $m_{n}=\mathcal{B}_{n}$. As equações de Andoyer devem ser satisfeitas com ambas as massas, assim considere as equações de Andoyer com 
índices diferentes de $n$, que podem ser escritas na seguinte forma

$$
f_{i j}=\sum_{k \neq i, j, n} m_{k}\left(R_{i k}-R_{j k}\right) \Delta_{i j k}+m_{n}\left(R_{i n}-R_{j n}\right) \Delta_{i j n}=0
$$

para todo $0<i<j<n$. Para os valores de massa fixados acima teremos,

$$
f_{i j}=\sum_{k \neq i, j, n} m_{k}\left(R_{i k}-R_{j k}\right) \Delta_{i j k}+\mathcal{A}_{n}\left(R_{i n}-R_{j n}\right) \Delta_{i j n}=0
$$

para todo $0<i<j<n \mathrm{e}$

$$
f_{i j}=\sum_{k \neq i, j, n} m_{k}\left(R_{i k}-R_{j k}\right) \Delta_{i j k}+\mathcal{B}_{n}\left(R_{i n}-R_{j n}\right) \Delta_{i j n}=0
$$

para todo $0<i<j<n$. Se subtrairmos (6.25) de (6.26), temos

$$
\left(\mathcal{A}_{n}-\mathcal{B}_{n}\right)\left(R_{i n}-R_{j n}\right) \Delta_{i j n}=0
$$

desde que consideramos $\mathcal{A}_{n} \neq \mathcal{B}_{n}$, esta equação se verifica se, e somente se,

$$
\left(R_{i n}-R_{j n}\right) \Delta_{i j n}=0 .
$$

Note que as equações em (6.27) são exatamente as mesmas daquelas vistas na provado Teorema 6.3.2, assim teremos as mesmas implicações geométricas deste Teorema. Ou seja, os vetores posição dos corpos com índices de 1 até $(n-1)$ tem que estar inscritos numa circunferência com centro em $r_{n}$ e mais que isto o centro de massa dos $(n-1)$ corpos deve coincidir com $r_{n}$. Vide prova dos Lemas 6.3.4 e 6.3.5. Isto finda a prova do teorema.

Este último resultado mostra que as únicas configurações centrais planares nas quais podemos variar continuamente o valor de uma das massas, mantendo fixas as posições e as massas dos demais corpos, são precisamente as configurações centrais planares $(n, 1)$-empilhadas. 
O mesmo resultado vale para configurações espaciais nas quais podemos variar continuamente uma massa. Isto é, vale o seguinte resultado.

Teorema 6.4.2 Considere uma configuração central espacial formada por $n$ corpos. Se podemos variar continuamente uma das massas mantendo as posições e demais massas fixas, então a configuração é uma configuração central espacial $(n, 1)$-empilhada.

Prova: Sem perda de generalidade vamos supor que a massa que pode ser variada é a de índice $n, m_{n}$. Considere dois valores diferentes para esta massa $m_{n}=\mathfrak{A}_{n}$ e $m_{n}=\mathfrak{B}_{n}$. As equações de Andoyer devem ser satisfeitas com ambas as massas, assim considere as equações de Andoyer com índices diferentes de $n$, que podem ser escritas na seguinte forma

$$
f_{i j l}=\sum_{k \neq i, j, l, n} m_{k}\left(R_{i k}-R_{j k}\right) \Delta_{i j l k}+m_{n}\left(R_{i n}-R_{j n}\right) \Delta_{i j l n}=0
$$

para todo $0<i<j<n$. Para os valores de massa fixados acima teremos,

$$
f_{i j l}=\sum_{k \neq i, j, l, n} m_{k}\left(R_{i k}-R_{j k}\right) \Delta_{i j l k}+\mathfrak{A}_{n}\left(R_{i n}-R_{j n}\right) \Delta_{i j l n}=0
$$

para todo $0<i<j<n$ e

$$
f_{i j l}=\sum_{k \neq i, j, l, n} m_{k}\left(R_{i k}-R_{j k}\right) \Delta_{i j l k}+\mathfrak{B}_{n}\left(R_{i n}-R_{j n}\right) \Delta_{i j l n}=0
$$

para todo $0<i<j<n$. Se subtrairmos (6.28) de (6.29), temos

$$
\left(\mathfrak{A}_{n}-\mathfrak{B}_{n}\right)\left(R_{i n}-R_{j n}\right) \Delta_{i j l n}=0
$$

desde que consideramos $\mathfrak{A}_{n} \neq \mathfrak{B}_{n}$, esta equação se verifica se, e somente se,

$$
\left(R_{i n}-R_{j n}\right) \Delta_{i j l n}=0
$$

Note que as equações em (6.30) são exatamente as mesmas daquelas vistas na prova do Teorema 
6.3.6, assim teremos as mesmas implicações geométricas deste Teorema. Ou seja, os vetores posição dos corpos com índices de 1 até $(n-1)$ tem que estar inscritos numa esfera com centro em $r_{n}$. Vide prova do Lema 6.3.8. Isto finda a prova do teorema.

Este último resultado mostra que as únicas configurações centrais espaciais nas quais podemos variar continuamente o valor de uma das massas, mantendo fixas as posições e as massas dos demais corpos, são precisamente as configurações centrais espaciais $(n, 1)$-empilhadas.

Os Teoremas 6.4.1 e 6.4.2 reforçam a importância do estudo das configurações centrais cocirculares e coesféricas. Existem várias questões em aberto por exemplo:

- Problema 1: Dada uma configuração central cocircular será que existem outras posições possíveis para colocar estas massas e ainda ter uma configuração central cocircular? Em outras palavras, uma configuração central cocircular é única?

- Problema 2: Roberts e Cors, vide [44], mostraram que numa configuração cocircular com os corpos ordenados ciclicamente, se fixarmos a maior massa no índice 1, são condições necessárias as seguintes desigualdades

$$
m_{1} \geq m_{2} \geq m_{4} \geq m_{3}
$$

Nesta linha, que propriedades devem ter $n$ massas para que seja possível obtermos uma configuração central inscritível?

- Problema 3: Roberts e Cors, vide [44], mostraram que o maior ângulo entre os vetores posição de uma configuração central cocircular é menor que $144^{\circ}$. No entanto, existem algumas evidências numéricas de que o maior ângulo seja menor que $120^{\circ}$. É possível obter uma prova analítica para esta afirmação?

- Problema 4: Para as configurações planares, conjectura-se que as únicas configurações centrais cocirculares com centro de massa no centro da circunferência são os polígonos regulares, vide 6.3.1. E para o caso espacial? 
Uma questão que está sendo estudada agora é quais são as configurações centrais nas quais podemos variar continuamente os valores de massa de dois corpos, mantendo fixas as posições e as massas dos demais corpos. Não há um resultado completo mas conjecturamos o seguinte,

Conjectura 6.4.3 Não existe configuração central planar não colinear na qual seja possível variar continuamente dois valores de massa, mantendo fixas as posições e demais massas.

Mais uma questão de interesse é quais são as configurações centrais nas quais podemos variar os valores de $k$ massas, mantendo fixas as posições e demais massas?

\subsection{Configurações Centrais Empilhadas Fracas}

Para finalizar, uma pergunta acerca das configurações centrais empilhadas, que seria um caso mais fraco daquele definido acima.

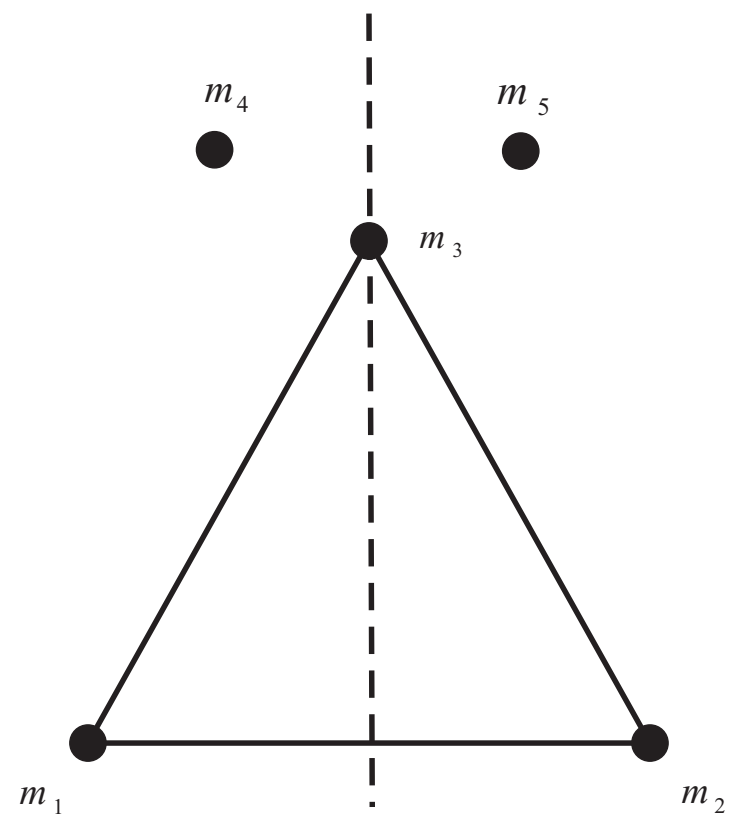

Figura 6.9: Configuração de Mello, Llibre e Perez-Chavela.

Definição 6.5.1 Uma configuração central será chamada de $(n, k)$-empilhada fraca, se podemos remover $k$ corpos, (os $k$ com maiores índices, por convenção), de tal forma que existam $(n-k)$ corpos, não necessariamente com os mesmos valores das massas de antes, de modo que mantendo as posições e escolhendo novas massas ainda tenhamos uma configuração central. 
Esta pergunta é motivada do estudo das configurações centrais de cinco massas no plano feita por Mello, Llibre e Perez-Chavela. Na figura 6.9 podemos retirar os dois corpos fora do triângulo equilátero que ainda teremos uma configuração central. Isto é, o caso estudado por eles é uma configuração central $(5,2)$-empilhada. Poderia ser uma configuração central $(5,1)$-empilhada? A resposta claramente é não, pelo Teorema 6.1.2 provado acima. Mas, retirando o corpo de massa $m_{3}$ teríamos que a configuração geométrica é um trapézio isósceles, que sob certas condições é uma configuração central.

Seria interessante um estudo dos casos mais simples. Um resultado que sai facilmente é para quatro corpos: a única configuração central planar, não colinear, $(4,1)$-empilhada fraca é o triângulo equilátero com corpos de massas iguais nos seus vértices e uma massa arbitrária no baricentro geométrico do triângulo. Isto resulta do Teorema 5.2.6 nos dizer que a única configuração central planar não colinear de três corpos é o triângulo equilátero. 


\section{Referências Bibliográficas}

[1] A. Albouy, V. Kaloshin, Finiteness of central configurations of five bodies in the plane, aceito para publicação no Annals of Mathematics.

[2] M. Alonso E E. Finn, Física um Curso Universitário, vol. 1, Edgard Bluscher, São Paulo (1972)

[3] M. H. Andoyer, Sur l'équilibrie relatif de n corps, Bull. Astron. vol. 23 p. 50, (1906).

[4] J. Bernat, J. Llibre e E. Pérez-Chavela, On the planar central configurations of the 4-body problem with three equal masses, Dynam. Contin. Discrete Impuls. Systems, vol. 16, 1-13, (2009);

[5] H. Bruns, Über des integrales der Vielkörperproblem, Acta Mathematica, vol. 11, (1887).

[6] N. CopéRnico, De revolutionibus orbium coelestium, (1543).

[7] L. Euler, De moto rectilineo trium corporum se mutuo attahentium, Novi Comm. Acad. Sci. Imp. Petrop. vol. 11, 144-151, (1767).

[8] N. FAyÇAL, On the classification of pyramidal central configurations. Proc. Am. Math. Soc., vol. 124, 249, (1996).

[9] D. Figueiredo e A. Neves, Equações Diferenciais Aplicadas, Coleção Matemática Universitária - IMPA, Rio de Janeiro, (2005).

[10] Y. Hagihara, Celestial Mechanics, vol 1, MIT Press, Massachusetts, (1970).

[11] M. Hampton, Co-circular central configurations in the four-body problem, Proc. EQUADIFF 2003, 993-998, (2004).

[12] M. Hampton, R. Moeckel, Finiteness of relative equilibria of the four-body problem, Invent. Math., vol. 163, 289-312, (2006).

[13] M. Hampton, Stacked central configurations: new examples in the planar five-body problem, Nonlinearity 18, 2299-2304, (2005).

[14] M. Hampton, M. Santoprete, Seven-body central configurations: a family of central configurations in the spatial seven-body problem, Celestial Mech. Dyn. Astr. 99, 293-305, (2007).

[15] J. KePler, Astronomia nova, (1610). 
[16] J.KePLer, Harmonice Mundi, (1614).

[17] E. Julliard-Toseal, Bruns' Theorem: the proof and some generalizations, Celestial Mechanics and Dynamical Astronomy, vol. 76, 241-281, (2000).

[18] J.L. Lagrange, Essai sur le problème de trois corps, Ouvres, vol. 6, Gauthier-Villars, Paris, $(1873)$.

[19] E. S. LEAndro, Finitness and bifurcations of some symmetrical classes of central configurations, Arch. Ratio. Mech. Anal., vol. 167, (2003).

[20] E. Lifshitz, L. Landau, Mechanics, $3^{r d}$ edition, Butterworth-Heinemann, (1982).

[21] G.G. Luther And W.R. Towler, Phys. Rev. Let., vol. 48, 121 (1982).

[22] C. McCord, Planar central configuration estimates in the N-body problem, Erg. Teo. and Dyn. Sys., vol. 16, 1059 - 1070, (1996).

[23] L. F. Mello, A. C. Fernandes, New classes of central Configurations in the spatial (n+3)body problem, Nonl. An. Real Wor. App., vol. 12, 723-730, (2009).

[24] L.F. Mello E A.C. Fernandes, Configurações centrais planares encaixantes, Revista Brasileira de Ensino de Física vol. 29, 385 (2007).

[25] L. F. Mello, A. C. Fernandes, New spatial central configurations in the 5-body problem, An. Acad. Bras. Ciênc, vol. 83, (2011).

[26] L. Mello; F. Chaves; A. C. Fernandes, Configurações centrais planares do tipo pipa. Revista Brasileira de Ensino de Física, vol. 31, 1302, (2009).

[27] L. F. Mello, F. E. Chaves, A. C. Fernandes, B. A. Garcia, Stacked central configurations for the spatial six-body problem, J. Geo. and Phy., vol. 59, 1216-1226, (2009).

[28] L. F. Mello, J. Llibre, New central configurations for the planar 5-body problem, Celestial Mech. Dyn. Astr., vol. 100, 141-149, (2007).

[29] L. F. Mello, J. Llibre, E. Perez-Chavela, New stacked central configurations for the planar 5-body problem, Celestial Mech. Dyn. Astr., vol. 110, 43-52, (2011).

[30] L. F. Mello, A.C. Fernandes, Co-circular and co-spherical kite central configurations, Qual. Teo. Dyn. Sys., vol. 10, 1-13, (2011).

[31] R. Moeckel, Celestial Mechanics Especially Central Configurations, Notas manuscritas de uma curso em Triesti, Itália (1994). URL: http://www.math.umn.edu/ rick/notes/Notes.html

[32] R. Moeckel, On central configurations, Math. Z., vol. 205, 499-517 , (1990).

[33] C. Moore, Braids in classical gravity, Phys. Rev. Let., vol. 70, (1993).

[34] F. Moulton, An Introduction to Celestial Mechanics, Dover Publications, (1984).

[35] I. Newton, Philosophia Naturalis Principia Mathematica, Royal Society, London, (1687). 
[36] D. SAARI, On the role and properties of central configurations. Celestial Mech. Dyn. Astr., vol. 21, 9-20, (1980).

[37] D. SAARI, Collisions, Rings and Other Newtonian N-Body Problems, CBMS, vol. 104, (2005).

[38] D. SAARI, Collisions, Rings and Other N-Body Problems. CBMS, vol. 104, (2000).

[39] S. Smale, Topology and Mechanics I, Inv. Mathe., vol. 11, 45-64, (1970).

[40] S. Smale, Mathematical problems for the next century, Math. Intell., vol. 20, 7-15, (1998).

[41] P. Smith, R. Smith, Mechanics; $2^{\text {nd }}$ edition, John Wiley \& Sons, New York, (1990).

[42] J. Sotomayor, Lições de Equações Diferenciais Ordinárias, Rio de Janeiro: IMPA - Projeto Euclides, (1979).

[43] G. Roberts, A continuum of relative equilibria in the five-body problem, Physica D, vol. 127, 141-145, (1999).

[44] G. Roberts, J. Cors, Cyclic central configurations in the four-body problem, submetido a Nonlinearity.

[45] C. Vidal, G. Renildo, Homografic solutions in the n-body problem. CUBO, vol. 6, 185$207,(2004)$.

[46] S. Volchan, Uma Introdução à Mecânica Celeste, P. matemáticas - IMPA, (2007).

[47] A. Wintner, Analytical Foundations of Celestial Mechanics, Princeton University Press, (1941).

[48] S. Zang, Q. Zhou, Double pyramidal central configurations,Phys. Lett. A 281, 240-248, (2001). 


\section{Índice Remissivo}

Aplicação Energia-Momento, 47

Centro de Massa, 18

Classes de configurações centrais, 55

Configuração Central, 32

Configuração central, 53

Configuração Central Empilhada, 123

Configuração Central Empilhada Fraca, 153

Configuração Central Piramidal, 137

Conjunto colisão, 17

Energia Cinética, 18

Energia Potencial, 18

Energia Total, 19

Equações de Andoyer, 58

Identidade de Lagrange Jacobi, 22

Lei da gravitação de Newton, 2

Massa Total, 18

Momento Angular Total, 19

Momento Linear Total, 18

Pontos Críticos da Superfície Energia-Momento, 47

Problema de $n$ corpos, 21

Referencial Baricêntrico, 22

Solução de Equilíbrio Relativo, 34

Solução Homográficas, 40

Soluções Homotéticas, 31

Teorema de Bruns, 21 